\title{
Adaptive Control and Control Allocation for Spacecraft Formation Flying under Perturbations, Uncertainties, and Faults
}

\author{
by \\ Raha Hojjati
}

A thesis submitted to the Faculty of Graduate and Postdoctoral Affairs

in partial fulfillment of the requirements for the degree of

\section{Master of Applied Science \\ in \\ Aerospace Engineering}

\author{
Carleton University \\ Ottawa, Ontario
}

(C) 2020

Raha Hojjati 
This work is dedicated to my grade 4 math teacher.

For all the homework she gave us. The 9 year old me hated it. But today, I appreciate the homework.

For it made understand the importance of hard work and gave me the math foundation I needed to succeed in engineering.

Thank you. 


\section{Abstract}

Spacecraft formation flying has been identified as an enabling technology. Researchers are focusing lots of efforts towards the development of autonomous control algorithms. Specifically, control laws are responsible for actuating the thrusters of the chaser spacecraft such that a relative desired trajectory is kept between the chaser and the target spacecraft. However, this poses a significant challenge as the formation experiences highly nonlinear dynamical effects as well as several external perturbations, which will cause both spacecraft to drift apart.

Keeping a tight formation is key to success for spacecraft formation flying missions. This research addresses fault tolerant control laws for spacecraft formation flying such that the chaser can accurately track a desired relative trajectory regardless of thruster faults, dynamical uncertainties, and perturbations. A controller based on simple adaptive control theory is tested and compared to three other control laws in numerical simulation using MATLAB/Simulink. The other three control laws are two types of nonsingular terminal sliding mode control law and an optimal sliding mode control law.

All control laws are tested for three types of actuator failures: loss of effectiveness, stuck actuators (locked in place), and total failure. Additionally, the performance of the control laws is investigated under nominal conditions and extra perturbations. From simulation, the conclusion can be made that simple adaptive control outperforms the other control laws in all but the locked in place scenario. That is, it has the fasted rate for recovery after the faults and the lowest steady state error. Optimal sliding mode control also has the same steady state error as simple adaptive control. For the locked in place scenario, at the cost of more control force, simple adaptive control has the fastest recovery rate. For the nominal case, simple adaptive control has the fastest transient response while requiring the least amount of control force. For the extra perturbation scenario, since simple adaptive control is not a model based control law, its performance is not affected by the perturbations unlike the three other control laws.

Moreover, simple adaptive control is implemented for an over actuated system. Two control allocation algorithm based on optimization techniques are used to distribute the control signals among the healthier actuators. These algorithms are weighted pseudo inverse and a closed loop quadratic programming algorithm. Given various fault scenarios, it is observed that quadratic programming has a better performance than the weighted pseudo inverse algorithm. 


\section{Acknowledgements}

First and foremost I would like to thank my supervisor Dr. Steve Ulrich for his support throughout my studies at Carleton University. I could have not asked for a better supervisor to guide me throughout my graduate studies.

I would like to thank my parents Ali and Nasim for all they have done for me throughout my life. I would have not been where I am today without their hard work and sacrifices.

And of course many thanks to my boyfriend, Ehab for editing my chapters and being patient and understanding with me during my graduate studies.

Last but not least I would like to thank all my friends for their support and encouragement and to all the teachers and professors that I have had the privilege to be educated by. For them believing in me and pushing me to become the person I am today.

This research was financially supported in part by Natural Sciences and Engineering Research Council of Canada through the Canada Graduate Scholarship as well as Carleton University and the Department of Mechanical and Aerospace Engineering entrance scholarship and Teaching Assistantships. 


\section{Table of Contents}

Abstract

Acknowledgements $\quad$ iv

List of Figures $\quad x$

List of Tables $\quad$ xi

List of Acronyms xii

Nomenclature $\quad$ xiv

1 Introduction 1

1.1 Motivation . . . . . . . . . . . . . . . . . . . 1

1.2 Problem Statement . . . . . . . . . . . . . . . . . . . 2

1.3 Previous Work . . . . . . . . . . . . . . . . . . . 3

1.4 Thesis Objective . . . . . . . . . . . . . . . . 7

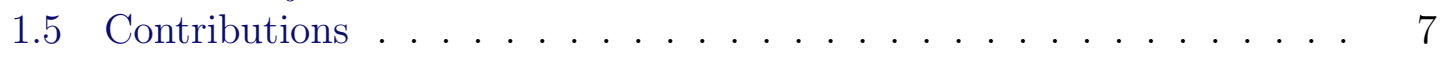

1.6 Thesis Organization . . . . . . . . . . . . . . . . . . 9

2 Spacecraft Formation Flying $\quad 10$

2.1 Spacecraft Formation Flying . . . . . . . . . . . . . . . 11

2.2 Kinematics and Dynamics . . . . . . . . . . . . . . . . 12

2.2.1 Angular Velocity and Derivative of a Vector . . . . . . . . 14

2.2.2 Rigid Body Dynamics . . . . . . . . . . . . . . . . 16

2.3 Attitude Representation . . . . . . . . . . . . . . . . . . . . . . . . . 19

2.3.1 Direction Cosine Matrix . . . . . . . . . . . . . . . . . . . 19

2.3 .2 Euler Axis . . . . . . . . . . . . . . . . . . . . . . 20

2.3 .3 Quaternions . . . . . . . . . . . . . . . . . . . . . 21

2.4 Two Body Problem . . . . . . . . . . . . . . . . . . . . . . 23

2.5 Frames of Reference. . . . . . . . . . . . . . . . . . 25

2.5.1 Earth-Centered Inertial (ECI) Reference Frame . . . . . . 25 
2.5.2 Perifocal Reference Frame . . . . . . . . . . . . . . . . 26

2.5.3 Local-Vertical Local-Horizontal (LVLH) Reference Frame . . . 27

2.5.4 Body Fixed Reference Frame . . . . . . . . . . . . . . . . . . 28

2.6 Classical Orbital Elements . . . . . . . . . . . . . . . . . . . 29

2.6.1 Position and Velocity in ECI . . . . . . . . . . . . . 31

2.7 Perturbations . . . . . . . . . . . . . . . . . . . 33

2.7.1 Gravity Perturbations due to Earth's Oblateness . . . . . . . . 33

2.7.2 Third Body Perturbations . . . . . . . . . . . . . 35

2.7.3 Aerodynamic Drag . . . . . . . . . . . . . . . 39

2.7.4 Solar Radiation Pressure . . . . . . . . . . . . . . . . . . . . . 42

2.8 Spacecraft Formation Flying Dynamics . . . . . . . . . . . . . . . . . . . . 44

2.9 State Space Representation . . . . . . . . . . . . . . . . . . . . 47

2.10 Plant Overview . . . . . . . . . . . . . . . . . . . . 48

2.11 Fault Modelling . . . . . . . . . . . . . . . . . 50

3 Nonsingular Terminal Sliding Mode Control 51

3.1 Introduction . . . . . . . . . . . . . . . . . . . 52

3.2 Tracking Error . . . . . . . . . . . . . . . . . . . . . . 53

3.3 Control Law . . . . . . . . . . . . . . . . . . . . . . . . . . . . . . . . . . . . . . . 53

3.4 Stability Analysis . . . . . . . . . . . . . . . . 55

3.5 The Chattering Problem . . . . . . . . . . . . . . . 57

3.6 Modified Nonsingular Terminal Sliding Mode Control . . . . . . . . . 58

3.7 Finite Time Analysis . . . . . . . . . . . . . . . . . . . . . . . . 59

4 Optimal Sliding Mode Control $\quad 60$

4.1 SFF Dynamics Modification . . . . . . . . . . . . . . . 61

4.2 Tracking Error . . . . . . . . . . . . . . . . . . 61

4.3 Optimal Control Law . . . . . . . . . . . . . . . . . . . . . 62

4.4 Integral Sliding Mode Control . . . . . . . . . . . . . . . . 63

4.5 Stability Analysis . . . . . . . . . . . . . . . . 65

5 Simple Adaptive Control $\quad 67$

5.1 Ideal Model . . . . . . . . . . . . . . . . . . . . . . . . 68

5.2 Control Law . . . . . . . . . . . . . . . . . . . . . . . . . . . . . . . . . . . 70

5.3 Almost Strictly Passive Plant . . . . . . . . . . . . . . . . . . . . . . . . . . . 72

5.4 Ideal Plant . . . . . . . . . . . . . . . . . . . . . . 75

5.5 Stability Analysis . . . . . . . . . . . . . . . . 77

6 Weighted Pseudo Inverse $\quad 79$

6.1 Introduction to Control Allocation . . . . . . . . . . . . . . . . 79

6.2 Problem Formulation . . . . . . . . . . . . . . . . . . . 80

6.3 Optimization Problem . . . . . . . . . . . . . . . . 82 
7 Quadratic Programming $\quad 84$

7.1 Control Allocation Problem . . . . . . . . . . . . . . . . . . 85

7.2 Open Loop Control Allocation . . . . . . . . . . . . . . . . . . 86

7.3 Closed Loop Control Allocation . . . . . . . . . . . . . . . . . . 89

7.4 Modified Closed Loop Control Allocation . . . . . . . . . . . . . . . 93

8 Results 96

8.1 Introduction . . . . . . . . . . . . . . . . . 97

8.2 Desired Formation . . . . . . . . . . . . . . . . . . . . . . 99

8.3 Nominal Case . . . . . . . . . . . . . . . . . . . . . . . . 100

8.4 Extra Perturbations . . . . . . . . . . . . . . . . . . . . . . 104

8.5 Fault Case . . . . . . . . . . . . . . . . . . . . . . . 106

8.5.1 Total Loss of Actuator . . . . . . . . . . . . . . . . 106

8.5.2 Locked in Place . . . . . . . . . . . . . . . . . . . . . . 109

8.5.3 Partial Loss of Effectiveness . . . . . . . . . . . . . . . . 113

8.5.4 Ideal Model Reset for SAC . . . . . . . . . . . . . . . 116

8.6 Control Allocation . . . . . . . . . . . . . . . . . . . . . . 122

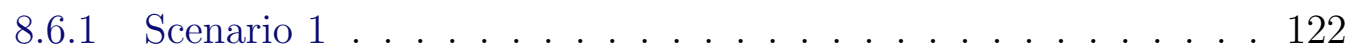

8.6 .2 Scenario $2 \ldots \ldots \ldots \ldots$. . . . . . . . . . . . 126

9 Conclusion 131

9.1 Thesis Summary . . . . . . . . . . . . . . . . . . . . . 131

9.2 Future Work . . . . . . . . . . . . . . . . . . . . . . 133 


\section{List of Figures}

2.1 SFF Diagram . . . . . . . . . . . . . . . . . . 11

2.2 Dynamics of a Rigid Body . . . . . . . . . . . . . . . 17

2.3 Principal Rotations . . . . . . . . . . . . . . . . . . . . . . 19

2.4 Euler Axis . . . . . . . . . . . . . . . . . . . . . . . . . . . 21

2.5 Attitude Kinematics and Dynamics . . . . . . . . . . . . . 22

2.6 Two Body Diagram . . . . . . . . . . . . . . . . . . . . . 24

2.7 ECI Reference Frame . . . . . . . . . . . . . . . . . . . 25

2.8 Perifocal Reference Frame . . . . . . . . . . . . . . . . 26

2.9 LVLH Reference Frame . . . . . . . . . . . . . . . . . . . . . 27

2.10 Body Fixed Reference Frame . . . . . . . . . . . . . . . . . . 28

2.11 Orbital Elements . . . . . . . . . . . . . . . . . . . . . . . . . 29

2.12 Geometrical Representation of a Spacecraft affected by Third Body

Perturbation, using the Sun as an example . . . . . . . . . . 35

2.13 Plant Overview . . . . . . . . . . . . . . . . . . . . 48

3.1 Phase Portrait of Sliding Mode control _ . . . . . . . . . . . . 52

3.2 NTSMC Block Diagram . . . . . . . . . . . . . . . . 53

3.3 Control Law Block Diagram . . . . . . . . . . . . . . . 55

3.4 Saturation Function . . . . . . . . . . . . . . . . . . . . 57

4.1 OSMC Block Diagram _. . . . . . . . . . . . . . . 63

4.2 Control Law Block Diagram . . . . . . . . . . . . . . . . . 64

5.1 Ideal Model Representation . . . . . . . . . . . . . . . . . . . 68

5.2 Simple Adaptive Control Diagram . . . . . . . . . . . . . . . . . 71

6.1 Overall Control Allocation Strategy . . . . . . . . . . . . . . 80

6.2 Weighted Pseudo Inverse . . . . . . . . . . . . . . . . . . . 83

7.1 Closed Loop Control Allocation Diagram . . . . . . . . . . . . . 89

7.2 Modified Closed Loop Control Allocation Diagram . . . . . . . . . . . 93

8.1 PCO Formation . . . . . . . . . . . . . . . . . . . . . . . . 99

8.2 Position Error . . . . . . . . . . . . . . . . . . . 100 
8.3 Position Error Transient Response . . . . . . . . . . . . . . . . . 101

8.4 Steady State Position Error . . . . . . . . . . . . . . . . . . . 101

8.5 Control Force . . . . . . . . . . . . . . . . . . . . . . . 102

8.6 Control Force Transient Response . . . . . . . . . . . . . . . . 103

8.7 Steady State Control Force . . . . . . . . . . . . . . . . . . . . . 103

8.8 Position Error . . . . . . . . . . . . . . . . . . . . . . . . . 104

8.9 Control Force . . . . . . . . . . . . . . . . . . . 105

8.10 Steady State Control Force . . . . . . . . . . . . . . . . . 105

8.11 Faults Representation . . . . . . . . . . . . . . . 106

8.12 Position Error . . . . . . . . . . . . . . . . . . . 106

8.13 Position Error during Recovery . . . . . . . . . . . . . . . . 107

8.14 Position Error after Recovery . . . . . . . . . . . . . . . . 107

8.15 Control Force . . . . . . . . . . . . . . . . . . 108

8.16 Control Force during Recovery . . . . . . . . . . . . . . . . . . 108

8.17 Actuator Forces before Faults . . . . . . . . . . . . . . . . . . . 109

8.18 Faults Representation . . . . . . . . . . . . . . . 110

8.19 Position Error . . . . . . . . . . . . . . . . . . . . 110

8.20 Position Error during Recovery . . . . . . . . . . . . . . . . . . 110

8.21 Position Error after Recovery . . . . . . . . . . . . . . . . . . 111

8.22 Control Force . . . . . . . . . . . . . . . . . . . . . . . . . . . . . . . . . . . . . . . . . .

8.23 Control Force during Recovery . . . . . . . . . . . . . . . . . . . 112

8.24 Faults Representation . . . . . . . . . . . . . . . . . . 113

8.25 Position Error . . . . . . . . . . . . . . . . . . . . 113

8.26 Position Error during Fault . . . . . . . . . . . . . . . . . . 114

8.27 Control Force . . . . . . . . . . . . . . . . . . . . . . . . 114

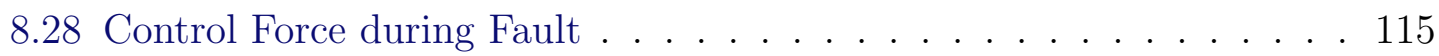

8.29 Ideal Model with Reset . . . . . . . . . . . . . . . . . 116

8.30 Position Error for the Total Loss Scenario . . . . . . . . . . . . . . . 117

8.31 Position Error Recovery for the Total Loss Scenario . . . . . . . . . . 117

8.32 Control Force for the Total Loss Scenario . . . . . . . . . . . . . . . . 118

8.33 Control Force during Recovery for the Total Loss Scenario . . . . . . 118

8.34 Position Error for the Locked in Place Scenario . . . . . . . . . . . . 119

8.35 Position Error Recovery for the Locked in Place Scenario . . . . . . . 120

8.36 Control Force for the Locked in Place Scenario . . . . . . . . . . . . . 120

8.37 Control Force during Recovery for the Locked in Place Scenario . . . 121

8.38 CA Reference Frames . . . . . . . . . . . . . . . . . . . . . . . 122

8.39 Fault Estimate . . . . . . . . . . . . . . . . . . 123

8.40 Actuator Forces . . . . . . . . . . . . . . . . . . . . . 123

8.41 Fault Estimate . . . . . . . . . . . . . . . . . . . . . . . . . . . . . . . . . . . . . . . .

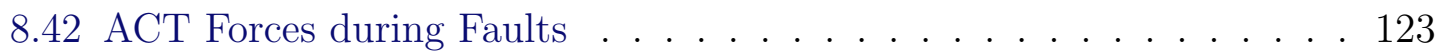

8.43 Actuator Force at 3805 seconds . . . . . . . . . . . . . . . . . . . . . 124 
8.44 Control Force . . . . . . . . . . . . . . . . . . . . . . . . . 124

8.45 Control Force during Fault . . . . . . . . . . . . . . . . . . . 125

8.46 Position Error . . . . . . . . . . . . . . . . . . . . . . 125

8.47 Fault Estimate . . . . . . . . . . . . . . . . . . 126

8.48 Actuator Forces . . . . . . . . . . . . . . . . . . . . . . 126

8.49 Fault Estimate . . . . . . . . . . . . . . . . . . . . . . 127

8.50 ACT Forces during Faults . . . . . . . . . . . . . . . 127

8.51 Actuator Forces at 4205 seconds . . . . . . . . . . . . . . . 127

8.52 Control Force . . . . . . . . . . . . . . . . . . . . . . . . . . . . . 128

8.53 Control Force during Fault . . . . . . . . . . . . . . . . . 128

8.54 Position Error . . . . . . . . . . . . . . . . . . . . . . . . . . . 129

8.55 CLCA vs. MCLCA . . . . . . . . . . . . . . . 130 


\section{List of Tables}

2.1 Coefficients for Calculating the Position of the Moon . . . . . . . 38

2.2 Exponential Atmospheric Model . . . . . . . . . . . . . . . 41

8.1 Orbital and System Parameters . . . . . . . . . . . . . . . 97

8.2 Terminal Sliding Mode Control Parameters . . . . . . . . . . . . . . 97

8.3 OSMC Parameters . . . . . . . . . . . . . . . . . . . 97

8.4 SAC Parameters . . . . . . . . . . . . . . . . . . . . . . . . . . . . . . . . . . . . . 98

8.5 CLCA Parameters ....................... 98 


\section{List of Acronyms}

$\begin{array}{ll}\text { ASP } & \text { Almost strictly passive } \\ \text { AU } & \text { Astronomical unit } \\ \text { CA } & \text { Control allocation } \\ \text { CanX } & \text { Canadian Advanced Nanosatellite eXperiment } \\ \text { CLCA } & \text { Closed loop control allocation } \\ \text { DCM } & \text { Direction Cosine Matrix } \\ \text { ECI } & \text { Earth-centered inertial } \\ \text { ESA } & \text { European Space Agency } \\ \text { FUSE } & \text { Far Ultraviolet Spectroscopic Explorer } \\ \text { GNC } & \text { Guidance, navigation, control } \\ \text { ISMC } & \text { Integral sliding mode control } \\ \text { JD } & \text { Julian date } \\ \text { LQR } & \text { Linear quadratic regulator } \\ \text { LVLH } & \text { Local-vertical local-horizontal } \\ \text { MCLCA } & \text { Modified closed loop control allocation } \\ \text { MNTSMC } & \text { Modified nonsingular terminal sliding mode control } \\ \text { MRAC } & \text { Model reference adaptive control } \\ \text { NASA } & \text { National Aeronautics and Space Administration } \\ \text { NTSMC } & \text { Nonsingular terminal sliding mode control } \\ \text { OSMC } & \text { Optimal sliding mode control } \\ \text { PDS } & \text { Positive definite symmetric } \\ \text { PID } & \text { Proportional-integral-derivative } \\ \text { PROBA } & \text { Project for On-Board Autonomy } \\ \text { QP } & \text { Quadratic programming } \\ \text { RAAN } & \text { Right ascension of the ascending node } \\ \text { SAC } & \text { Simple adaptive control } \\ \text { SFF } & \text { Spacecraft formation flying } \\ \text { SMC } & \text { Sliding mode control } \\ \text { SRP } & \text { Solar radiation pressure } \\ \text { SVD } & \text { Singular value decomposition } \\ \text { TPF } & \text { Terrestrial Planet Finder } \\ & \end{array}$


TSM Terminal sliding mode

UTC Universal time coordinate

WPI Weighted pseudo inverse 


\section{Nomenclature}

\section{Roman Symbols}

\begin{tabular}{ll}
\hline & \\
$\vec{a}$ & semi-major axis \\
$\vec{a}$ & acceleration vector \\
$A$ & surface area of spacecraft exposed to atmosphere/solar radiation \\
$\boldsymbol{A}$ & state matrix \\
$\boldsymbol{a}_{p}$ & perturbing acceleration \\
$\overrightarrow{\mathcal{B}}$ & reference vectrix defining the body fixed reference frame \\
$\boldsymbol{B}$ & input matrix \\
$\boldsymbol{C}$ & output matrix \\
$\boldsymbol{C}_{b a}$ & rotation matrix, rotation of frame $b$ with respect to frame $a$ \\
$C_{D}$ & drag coefficient \\
$\vec{c}_{D}$ & aerodynamic center of pressure \\
$\boldsymbol{C}_{I P}$ & rotation from perifocal reference frame to ECI reference frame \\
$C_{R}$ & radiation pressure coefficient \\
$\vec{c}_{S}$ & solar radiation center of pressure \\
$\boldsymbol{D}$ & actuator distribution matrix \\
$e$ & orbit eccentricity \\
$\boldsymbol{e}$ & position error \\
$E$ & eccentric anomaly \\
$\boldsymbol{E}$ & actuator effectiveness matrix \\
$\hat{\boldsymbol{E}}$ & estimate of actuator effectiveness matrix \\
$\boldsymbol{e}_{x}$ & SAC state error \\
$\boldsymbol{e}_{y}$ & SAC tracking error \\
$\vec{f}$ & force vector \\
$\overrightarrow{\mathcal{F}}$ & reference frame \\
$\boldsymbol{F}$ & perturbing force \\
$\boldsymbol{G}$ & universal gravitational parameter \\
$\boldsymbol{G}$ & perturbation bound matrix for NTSMC \\
$\vec{h}$ & angular momentum vector \\
& \\
& \\
& \\
\hline &
\end{tabular}




\begin{tabular}{|c|c|}
\hline$i$ & orbit inclination \\
\hline $\boldsymbol{I}$ & identity matrix \\
\hline $\overrightarrow{\mathcal{I}}$ & reference vectrix defining the ECI Frame \\
\hline $\boldsymbol{J}$ & inertia matrix \\
\hline$J_{2}$ & second zonal harmonic coefficient of the gravitational potential \\
\hline$k$ & delay \\
\hline $\boldsymbol{K}$ & (M)NTSMC control gain \\
\hline $\boldsymbol{K}_{i}$ & SAC integral control gain \\
\hline $\boldsymbol{K}_{p}$ & SAC proportional control gain \\
\hline $\overrightarrow{\mathcal{L}}$ & reference vectrix defining the LVLH reference frame \\
\hline$m$ & mass \\
\hline$M$ & mean anomaly \\
\hline$M$ & a constant matrix such that $\boldsymbol{M} \boldsymbol{B}$ is nonsingular for OSMC \\
\hline$n$ & mean orbital motion \\
\hline$p$ & (M)NTSMC control parameter \\
\hline$p$ & semilatus rectum \\
\hline$\vec{p}$ & linear momentum vector \\
\hline $\overrightarrow{\mathcal{P}}$ & reference vectrix defining the perifocal reference frame \\
\hline $\boldsymbol{P}$ & solution to Riccati equation \\
\hline$P_{n}$ & associated Legendre function \\
\hline$P_{\odot}$ & solar radiation pressure at a distance of $1 \mathrm{AU}$ \\
\hline$q$ & (M)NTSMC control parameter \\
\hline$q$ & quaternion \\
\hline$Q$ & LQR control parameter minimizing tracking error \\
\hline$\vec{r}$ & position vector \\
\hline $\boldsymbol{R}$ & LQR control parameter minimizing control effort \\
\hline$R_{\oplus}$ & geocentric Earth radius \\
\hline$\vec{s}$ & distance from spacecraft to Sun \\
\hline$s$ & sliding surface \\
\hline$t$ & time \\
\hline$t_{p}$ & time of perigee passage \\
\hline $\boldsymbol{u}$ & control input vector \\
\hline $\boldsymbol{u}_{m}$ & reference model input signal \\
\hline$\vec{v}$ & velocity vector \\
\hline$\vec{v}_{r}$ & spacecraft velocity relative to atmosphere \\
\hline $\boldsymbol{W}_{0.1,2}$ & QP weighing positive definite matrix \\
\hline $\boldsymbol{x}$ & state vector \\
\hline $\boldsymbol{x}_{m}$ & reference model state vector \\
\hline$y$ & output vector \\
\hline $\boldsymbol{y}_{m}$ & reference model output vector \\
\hline
\end{tabular}




\title{
Greek Symbols
}

\author{
$\boldsymbol{\Gamma}_{I} \quad$ positive definite integral weighting matrix for $\mathrm{SAC}$ \\ $\Gamma_{P} \quad$ positive definite proportional weighting matrix for SAC \\ $\gamma \quad$ boundary layer for saturation for SMC \\ $\boldsymbol{\epsilon} \quad$ vector part of quaternion \\ $\zeta \quad$ reference model damping ratio \\ $\eta \quad$ scalar part of quaternion \\ $\boldsymbol{\eta} \quad$ (M)NTSMC positive definite control gain matrix \\ $\theta \quad$ true anomaly \\ $\boldsymbol{\lambda}$ OSMC control parameter \\ $\mu \quad$ gravitational parameter \\ $\rho \quad$ atmospheric density \\ $\vec{\rho} \quad$ distance from the origin of $\mathcal{F}_{B}$ to $d m$ \\ $\vec{\rho} \quad$ position vector of chaser with respect to target \\ $\dot{\vec{\rho}} \quad$ velocity of chaser with respect to target \\ $\vec{\tau} \quad$ torque \\ $\phi \quad$ Euler angle \\ $\Omega \quad$ right ascension of the ascending node \\ $\omega \quad$ orbit argument of perigee \\ $\vec{\omega} \quad$ angular velocity \\ $\omega_{n} \quad$ reference model natural frequency
}




\section{Superscripts}

\begin{tabular}{ll}
\hline-1 & inverse operator \\
+ & pseudo-inverse \\
$\mathrm{T}$ & transpose operator \\
$\mathrm{x}$ & skew-symmetric matrix \\
$\mathbf{S u b s c r i p t s}$ \\
\hline
\end{tabular}




\section{Chapter 1}

\section{Introduction}

\subsection{Motivation}

Spacecraft formation flying (SFF) has revolutionized space based operations and it has opened up a plethora of possibilities for the space industry. Because of this, $\mathrm{SFF}$ has become an interest to various space agencies such as National Aeronautics and Space Administration (NASA) and The European Space Agency (ESA). The first SSF attempt took place in 1962 with Soviet Union Vostok 4 and 5 missions [1]. NASA attempted its first autonomous SFF mission in 2001 [2,3]. There have been over $20 \mathrm{SFF}$ missions in the last two decades and over 10 missions are expected in the next nine years [4].

SFF is a technology where two or more spacecraft fly in a tightly controlled configuration and act as one system. Flying smaller and simpler spacecraft provides many advantages over flying one massive and expensive spacecraft. These advantages include but are not limited to cost, distribution of payload among multiple spacecraft, fuel consumption, and redundancy in the event of a failure [4]. Examples of SFF missions are ESA's XEUS [5] and Project for On-Board Autonomy-3 (PROBA-3) [6] missions, NASA's Terrestrial Planet Finder (TPF) [7,8] mission, and many others such as PRISMA [9], Orion [10], TechSAT 21 [11], Canadian Advanced Nanosatellite eXperiment 4 and 5 (CanX-4,-5) [1], AAReST [12], and TanDEM-X [13].

The AAReST mission consisted of two chasers and a target, where two 3U CubeSat nanosatellite chasers can autonomously dock and undock with a central $15 \mathrm{U}$ micro/nano satellite core. The two chasers are MirrorSats and they are used for high resolution imaging [12]. The objective of this mission could not have been achieved if one massive spacecraft was flown. Similarly, for TPF mission, the use of five spacecraft allowed for a better resolution of interferometer to find Earth-like planets $[7,8]$. Without SFF, a giant telescope had to be launched, with the possibility of required assembly in space.

Despite the discussed advantages, SFF comes with several challenges. One of 
the biggest challenges is the importance of having a reliable on-board guidance, navigation, and control (GNC) system that would allow precise formation throughout the mission's life. This is of importance as keeping a tight formation is key to success for these missions and a failure in the GNC system can lead to catastrophic disasters. For example, for the TPF mission, keeping a precise formation between the five spacecraft was a must for imaging; without the tight formation, the mission's objective could have not been achieved.

\subsection{Problem Statement}

Given the stringent control of relative distances, SFF missions are very sensitive to disturbances and uncertainties. Therefore, a successful SFF mission often requires control laws that are robust so that the formation can be maintained in the presence of external disturbances such as gravitational perturbation, aerodynamic drag, and solar radiation pressure.

For example, the TPF mission required a control accuracy of $2 \mathrm{~cm}$ for the formation [8]. For CanX-4 and CanX-5 missions, the size of the formation ranged from 500 to $1000 \mathrm{~m}$ and the goal of the mission was to demonstrate a relative position control accuracy of $1 \mathrm{~m}$ for at least 10 orbits [1]. For TanDEM-X, the distance between the two spacecraft varied from $150 \mathrm{~m}$ to a few kilometers. A control accuracy of $5 \mathrm{~m}$ in the cross-track and $30 \mathrm{~m}$ in the along-track was achieved, while the requirement was $28 \mathrm{~m}$ in the cross-track and $200 \mathrm{~m}$ in the along-track [13].

In view of the above, robust performance and fault tolerance of the controller is critical for a successful mission. Actuator failures can impact a mission in different ways. For example, the failure of one momentum wheel for the Ecostar 5 spacecraft interrupted the mission while the failure of three out of four thrusters for the Galaxy 8i mission shortened the mission's life [14]. On the other hand, the failure of a reaction wheel for GPS B11-08 spacecraft resulted in the mission's total loss [14]. Another example of missions impacted by actuators' failures is NASA's Lewis spacecraft. Lewis was launched on August 23, 1997 and a serious malfunction of the thrusters caused the mission to end three days after its launch [15].

For this reason, spacecraft are often equipped with multiple actuators for redundancy. The technique used to distribute the control effort to individual actuators in a specified manner is called control allocation (CA). For example, if one actuator fails, the control effort is redistributed among healthy ones. It should be noted that often, CA is separated from the baseline controller [16] and is designed based on optimization techniques [16,17]. An example of the CA is NASA's Far Ultraviolet Spectroscopic Explorer (FUSE) spacecraft. FUSE was equipped with four reaction wheels and three magnetic torquer bars for attitude control. Two of the reaction wheels failed two years into the mission's life due to excessive friction between the rotors and the wheel housings. NASA managed to regain the control of the spacecraft 
by using the two remaining reaction wheels and the magnetic torquer bars [18].

To summarize, a failure of an actuator could result in catastrophic accidents such as the loss of one or more spacecraft. Thus it is of great importance for autonomous control that these spacecraft carry on-board fault tolerant control algorithms that allow precise formation in the presence of external perturbations, various uncertainties, and actuator failures. This thesis is concerned with the development of adaptive control to cope with failures and uncertainties as well as combining adaptive control technique with cases where redundancy exists.

\subsection{Previous Work}

Development of fault tolerant control laws is valuable for many systems such as unmanned aerial vehicles, fighter jets, and spacecraft. This section highlights various fault tolerant control laws that have been proposed for SFF and spacecraft attitude control. This is followed by another section, which discusses the development of various optimization based techniques for control allocation.

\section{SFF Fault Tolerant Control Laws}

Only a limited number of control laws have been developed for fault tolerant SFF. For example, Gorard and Kumar [19] discuss an adaptive sliding mode control law (SMC) as well as an adaptive nonsingular terminal sliding mode control (NTSMC) for actuator failures. In this paper, the target spacecraft is on an unperturbed elliptical orbit; even though perturbations are taken into account for the chaser spacecraft, these perturbations are modelled as a sinusoidal function with a magnitude lower than what is experienced by low Earth orbiting satellites. Gorard and Kumar use full nonlinear translational dynamics to model the motion of the chaser spacecraft with respect to the target spacecraft. The NTSMC law yields better results than the SMC law for both nominal and fault cases. Godard and Kumar test their control laws for four types of failures. These are thruster degradation, stuck thrusters, total loss of thrusters, and position sensors jam. A thorough explanation of the three thruster failures will be presented in Sec. 2.11.

Cao et al. [20] also propose a sliding control mode called minimum sliding mode error feedback control for fault tolerant SFF. Their control law is based on NTSMC; however, they modify the nonlinear switching control aspect of SMC law. They also incorporate a model predictive algorithm to enhance the performance of the system. The only perturbation taken into account is $J_{2}$, and the target is on an almost circular orbit. The results show a good recovery from the faults. Cao et al. use the same three types of scenarios for modelling actuator failures as [19]: loss of effectiveness, stuck actuator, and total loss. 
Lee et al. take a different approach than Godard and Cao to implement a fault tolerant control law. Lee et al. [21] implement a super twisting sliding mode observer. They propose a fault detection estimation scheme and based on the faults, the controller can be reconfigured. Two types of faults are investigated: one for the $\mathrm{x}$-axis thruster and the other for the $\mathrm{y}$-axis thruster. Given the axis of failure, the state space representation, which is based on Hill's equations, is modified.

Thanapalan et al. [22] also use Hill's equations, that is linearized equations of motion, to model SFF. This paper uses reconfigurable position control based on model reference adaptive control, and reconfigurable attitude control based on quaternion feedback. Their control law adapts very well to actuator failures, but their control law has only been tested for degradation in actuator effectiveness. For position tracking, the controller gain is modified and for attitude tracking, the actuators effectiveness is reduced by $60 \%$.

\section{Dual Quaternions Based Control Laws}

Another set of papers also discuss fault tolerant control laws for SFF; however, their work in based on dual quaternions. Dual quaternions allow one to simultaneously control both the position and attitude of a spacecraft. An example of dual quaternions is presented in [23]. Dong et al. [23] discuss a terminal sliding mode control that uses dual quaternions in order to maintain the position and attitude of the chaser with respect to the target. Their control law has only been tested for partial failure of the actuators. External disturbance forces and torques are modelled as sinusoidal functions.

Gui and de Ruiter [17] propose a hybrid dual quaternion integral sliding mode control. This paper take into account gravity gradient and $J_{2}$ perturbations as well as other external disturbances. The chaser spacecraft in this paper is over-actuated, meaning that there is a redundancy in the system. This actuator redundancy allows the authors to use CA. The proposed law in [17] can handle stuck actuators and it only works if three control forces and three control torques remain available.

\section{Attitude Fault Tolerant Control Laws}

Unlike other control laws that discuss position tracking or simultaneous attitude and position tracking using dual quaternions, Zhu and Guo [24] have developed a fault tolerant control law for SFF for attitude tracking. This control law is based on graph theory and allows the chaser spacecraft to track the attitude and angular velocity of the leader in the presence of unknown time-varying inertia and external disturbances. In this paper, the attitude of four spacecraft is control with respect to the leader spacecraft. Each spacecraft is equipped with four actuators, and the control law has been tested for a scenario where two out of the four actuators fail. 
Even though fault tolerant control schemes for SFF have not been greatly focused on in the past, spacecraft attitude fault tolerant control laws have been an interest to many researchers. For example, Bustan et al. [25] propose a fault tolerant law, which is based on variable structure control. This control law has the capability of controlling the transient response of the closed loop.

Xiao et al. [26] design a nonlinear terminal sliding mode observer for actuator failure and saturation by only taking into consideration attitude measurements and Gui and Vukovich [27] propose an integral sliding mode controller for fault tolerant attitude control. In terms of actuator failures, [25] only models actuator degradation while $[26,27]$ model actuator degradation and bias torque.

\section{Simple Adaptive Control}

Majority of the papers discussed for fault tolerant systems use a type of sliding mode control (SMC). SMC is a model based control law that requires a perfect knowledge of the plant. If the plant is not accurate, the performance of the system will degrade significantly. A solution to this is using adaptive control laws. More specifically, simple adaptive control (SAC) theory can be implemented. Unlike SMC, SAC is not a model based control law and hence its performance will not be affected by the precision of the plant.

Various studies have developed fault tolerant control laws based on SAC. Matsuki et al. [28] propose a SAC law with a proportional-integral-derivative (PID) controller for a manned aircraft. Takase et al. [29] also discuss the implementation of SAC with a PID compensator. Another example of SAC is demonstrated in [30]. This paper presents a control law for a quadrotor helicopter. Chen et al. [30] use SAC with a linear quadratic optimal controller to design a fault tolerant control law. Belkharraz and Sobel [31], Cano and Sobel [32] propose SAC with a feedforward compensator to meet the almost strictly positive real condition. They present a numerical example of a F/A-18 aircraft in presence of lateral gusts and loss of effectiveness to demonstrate their control law.

All the papers mentioned above, [28-32], only test their control laws for loss of effectiveness scenario. For example, in [30], 50\% loss of effectiveness is considered for two out of four actuators while in [31], the control law is tested for $88 \%$ loss of effectiveness. To the best of the author's knowledge, SAC has never been tested for overactuated systems and never for cases where the actuators are stuck or totally lost. To add on, a fault tolerant controller based on SAC has never been studied for SFF. 


\section{Control Allocation}

As previously mentioned, $\mathrm{CA}$ is a technique used to distribute the control effort to individual actuators. CA is especially useful for overactuated systems that may experience failures.

One of the techniques extensively used for CA in faulty scenarios is called weighted pseudo inverse, also referred to as Inverse Dynamics Control Law [33,34]. Boskovic and Mehra [33] discuss using a multiple mode adaptive reconfigurable control approach. This control law works only if one actuator is totally damaged while others have lost their effectiveness. The controller has been tested using linearized dynamic equations for the F/A-18A aircraft. The control law has a limit; if two actuators stop functioning, the system will become unstable.

An improved weighted pseudo inverse algorithm with an anti-saturation controller is implemented in [35-37]. This CA algorithm takes into consideration actuator saturation, which prevents the actuators from saturating. This CA has been used with various baseline control laws and implemented for different systems. For example, Wang et al. [35] use model reference adaptive control as their baseline controller with CA that redistributes the control input into eight actuators in case of failures, without saturating the healthy ones. The same weighting algorithm is used in [36], but their control allocation is only used for redistributing the control effort in case an actuator saturates. This is done through implementing a $H_{\infty}$ controller. Both $[35,36]$ show simulations of a satellite launch vehicle to illustrate the proposed control law and CA technique.

Alwi and Edwards [38] use weighted pseudo inverse algorithm with a sliding mode control law. It was shown that during the faults, the control input was redistributed along healthy ones without compromising the performance of the system. Based on Alwi and Edwards findings, Shen et al. [39] modify this CA algorithm for fault tolerant spacecraft attitude control. Their CA algorithm considers the estimate of the effectiveness of the actuators in the constraint of the optimization problem. For spacecraft attitude control, typically the control effort is sent to three actuators but a redundant spacecraft has four actuators. The four reaction wheels experience partial loss of effectiveness or a total failure in [39]. Gui and de Ruiter [17] expand the CA in [39] such that it can be used for a total of eight actuators: four for position and four for attitude.

Another class of CA used is called quadratic programming. A quadratic programming algorithm has been proposed by Härkegård [40]. Hu et al. [41] expanded on this CA algorithm by implementing a closed loop control allocation for spacecraft

attitude control. It has been shown that the closed loop CA (CLCA) can reduce the allocation error under actuator uncertainties caused by misalignment and measurement errors, and can provide a better performance.

Li et al. [42] modified the CLCA algorithm such that it take faults into account. This CLCA algorithm was implemented with a NTSMC law. The CLCA algorithm 
was compared with a pseudo inverse algorithm and QP showed a superior performance. Qiao et al. [43] modify the CLCA algorithm in [41]. It was demonstrated that the MCLCA outperform the one in [41] when the system experiences actuator uncertainties such as misalignment. The control laws and the CA techniques in [41-43] were all implemented for spacecraft attitude control.

To the best of the author's knowledge, the CLCA algorithm based on quadratic programming has never been implemented for SFF or position tracking and the MCLCA has never been implemented for systems that experience faults. Additionally, CLCA has never been used with SAC as the baseline control law.

\subsection{Thesis Objective}

The objective of this thesis is to develop a fault tolerant control law for SFF based on SAC theory for a system with and without redundancy. For the first part of this thesis, SAC is compared to other control laws that have been implemented for SFF without a redundancy. This includes the work done by Godard and Kumar [19] as well as the work done by Cao et al. [20]. SAC is also compared with another control law called optimal sliding mode control (OSMC), proposed by Imani and Bahrami [44]. This control law is a combination of optimal control law and integral sliding mode control (ISMC). Various types of SMC have been used extensively for fault tolerant control laws and the goal is to determine whether SAC can outperform the others. Most of the research done has used either $J_{2}$ or unrealistic perturbation. In the numerical simulation, the nonlinear translational dynamics of SFF are implemented and exact equations are used to model various perturbations experienced by low Earth orbiting spacecraft.

The second part of this thesis focuses on various CA algorithms combined with SAC. Two quadratic programming closed loop CA are implemented based on the work done by $[42,43]$. These CA algorithms are then compared with a weighted pseudo inverse based on the work done by Shen et al. and Gui and de Ruiter $[17,39]$.

\subsection{Contributions}

- Implementing four control laws: NTSMC based on the work in [19], MNTSMC based on the work in [20], OSMC based on the work in [44], and finally SAC;

- Studying the performance of these four control laws for a nominal case under perturbations;

- Implementing various types of failures: loss of effectiveness, total failure, and stuck actuators; 
- Investigating the performance of the four control laws under the different faults in a perturbed environment; and

- Developing the weighting pseudo inverse algorithm based on the work done by $[17,39]$ and the CLCA quadratic programming based on the work by $[42,43]$ and comparing the performance of the two. 


\subsection{Thesis Organization}

The remainder of this thesis is divided into a number of chapters.

Chapter 2: Spacecraft Formation Flying provides the reader with a variety of concepts that are important to the thesis. This chapter introduces kinematics and dynamics which lay the foundation for developing equations of motion. This is followed by attitude representation, which will be required for CA implementation. Other topics discussed in this chapter are reference frames, orbital and attitude perturbations, and the derivation of the exact nonlinear equations of relative motion for SFF. The overview of the plant as well as fault modelling are also described in this chapter.

The definition of sliding mode control (SMC) and the implementation of NTSMC and MNTSMC is presented in Chap. 3: Nonsingular Terminal Sliding Mode Control. The control law, the stability proof based on Lyapunov second method, finite time analysis, as well the the chattering phenomena are explained in this chapter.

Chapter 4: Optimal Sliding Mode Control presents OSMC law. This chapter first begins by introducing the modifications that are required for the plant. This is then followed by the development of the tracking error, the definition of optimal control theory and integral sliding mode control, the development of OSMC, and concluded with the stability proof of the control law based on Lyapunov second method.

Chapter 5: Simple Adaptive Control introduces simple adaptive control theory. This chapter highlights the various concepts needed for SAC such as the definition of the desired and ideal model, the control law, and the stability proof based on Lyapunov second method and LaSalle's invariance principle for non-autonomous systems.

The next two chapters detail two different algorithms for CA. Chapter 6: Weighted Pseudo Inverse discusses a weighted pseudo inverse algorithms that has been extensively used in the past. This chapter beings by a brief introduction of the definition of CA, followed by the optimization problem for weighted pseudo inverse and its solution. This is followed by Chap. 7: Quadratic Programming, which introduces the quadratic programming CA. This chapter contains the solution to the optimization problem, the derivation of the closed loop CA, and its stability proof based on discrete-time systems theory. The modified closed loop CA for fault tolerance cases is also presented in this chapter, followed by its stability proof.

Chapter 8: Results presents the results of the thesis based on the simulations done in MATLAB/Simulink. This includes the comparison of the four control laws for nominal and three faults scenarios as well as the results for the CA techniques. This thesis ends with Chap. 9: Conclusion, which summarizes the research work completed and provides recommendations for future work. 


\section{Chapter 2}

\section{Spacecraft Formation Flying}

This chapter introduces the reader to the basic concepts that lay the foundation for the rest of this thesis. A variety of topics are covered in this chapter such as spacecraft formation flying (SFF) terminology, orbital and attitude dynamics, reference frames, orbital perturbations, the overview of the plant, and fault modelling.

Section 2.1: Spacecraft Formation Flying provides the SFF terminology that will extensively be used in this thesis. This is followed by Sec. 2.2: Kinematics and Dynamics. Kinematics is the study of motion without considering forces and torques, while dynamics is the study of forces, torques, and their effects on the motion. The kinematics section provides an overview of vectors and rotation matrices as well as the notations and conventions that will be used throughout this thesis. The definition of rigid body and Euler's law are given in the dynamics section. The next section, Sec. 2.3: Attitude Representation, describes the most important parameters that are used to express rotations in a three-dimensional space.

Section 2.4 Two Body Problem presents the two body equation of motion. The derivation of this equation is based on Newton's universal law of gravitation. Section 2.5: Frames of Reference highlights the important reference frames that are used throughout the thesis. Section 2.6: Classical Orbital Elements presents the six orbital elements and their importance as they are the simplest and easiest way to denote the spacecraft's position as well as the orbital plane's orientation and size.

This is followed by Sec. 2.7: Perturbations. This section defines the various orbital and attitude perturbations that are used in the MATLAB/Simulink numerical simulator. These perturbations include Earth oblateness, third body, aerodynamic drag, and solar radiation pressure. SFF equations of motion, which describe the motion of the chaser spacecraft with respect to the target, are presented in Sec. 2.8: Spacecraft Formation Flying Dynamics and their respective state space representation is given in Sec. 2.9: State Space Representation. This is followed by the overview of the plant in Sec. 2.10: Plant Overview.

Actuators can fail in different ways. For example, an actuator can lose its ef- 
fectiveness or it can get stuck at a certain value. In a worst case scenario, the actuator could totally fail and no longer provide thrust. These different scenarios and their implementation in the numerical simulator are discussed in Sec. 2.11: Fault Modelling.

\subsection{Spacecraft Formation Flying}

Spacecraft formation flying consists of two or more spacecraft, where a set of spacecraft follow a leader in a predefined formation. The primary spacecraft, the leader, is referred to as the target in this thesis. The target is controlled to a referenced orbit, and the follower spacecraft are controlled with respect to the target [45]. In this thesis, the follower spacecraft are referred to as the chaser. The SFF consists of two spacecraft in this thesis: a target and a chaser. An example diagram of SFF is shown in Fig. 2.1. Figure 2.1 shows an in-plane elliptical formation. The dynamics equations for SFF are presented in Sec. 2.8.

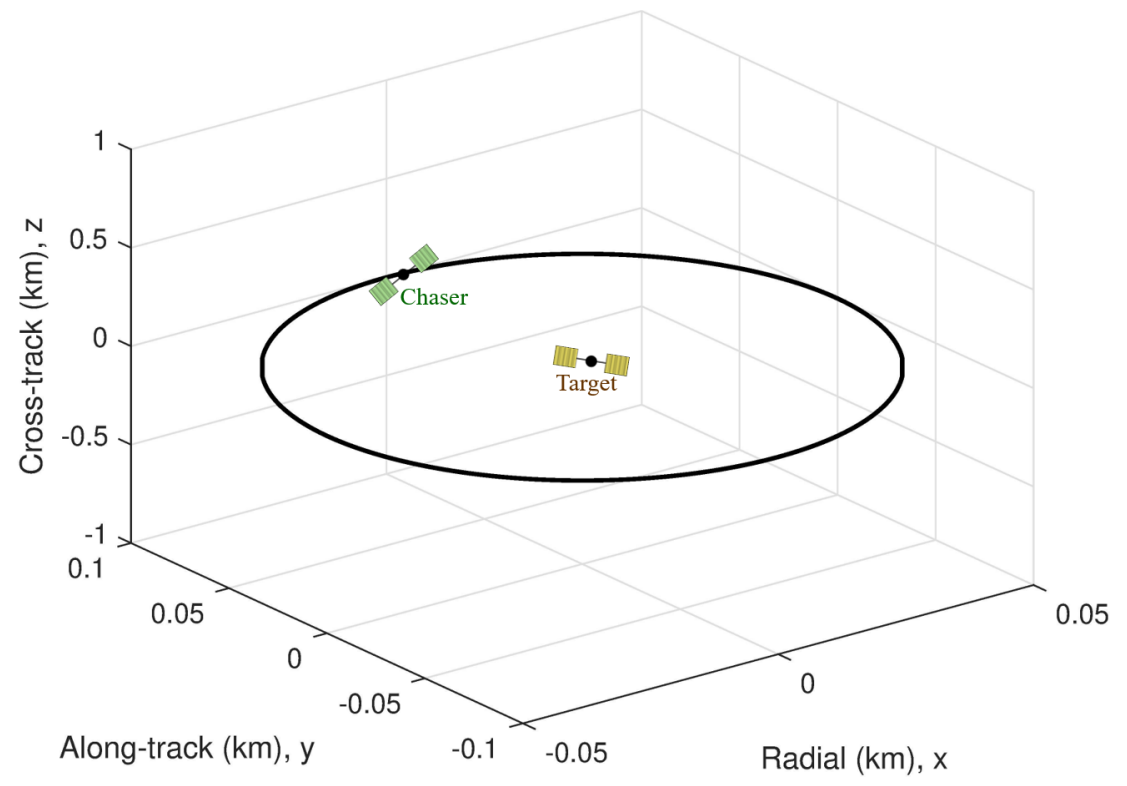

Figure 2.1: SFF Diagram 


\subsection{Kinematics and Dynamics}

This section provides the mathematical tools required to develop equations of motion. A vector is a three-dimensional quantity that has a magnitude and a direction [46]. Vectors are used to describe different quantities such as position, velocity, acceleration, force, momentum, torque, and angular velocity [47]. Vectors are independent of any reference frame [46].

For formulation of kinematics and dynamics, it is critical to be able to define the orientation of an object. Reference frames are used for this and allow one to express a vector in component form in a given reference frame.

A reference frame is indicated by three orthogonal unit vectors that follow the right hand rule [46]. For example, for reference frame one, denoted by $\mathcal{F}_{a}$, the three unit vectors are $\vec{a}_{x}, \vec{a}_{y}$, and $\vec{a}_{z}$. A vector in $\mathcal{F}_{a}$ reference frame can be expressed as $[46]$

$$
\begin{aligned}
\vec{u} & =u_{x, a} \vec{a}_{x}+u_{y, a} \vec{a}_{y}+u_{z, a} \vec{a}_{z} \\
& =\left[\begin{array}{lll}
\vec{a}_{x} & \vec{a}_{y} & \vec{a}_{z}
\end{array}\right]\left[\begin{array}{l}
u_{x, a} \\
u_{y, a} \\
u_{z, a}
\end{array}\right] \\
& =\overrightarrow{\mathcal{F}}_{a}^{T} \boldsymbol{u}_{a}
\end{aligned}
$$

Therefore, it can be concluded that the vector $\vec{u}$ in reference frame $\mathcal{F}_{a}$ can be expressed as $\overrightarrow{\mathcal{F}}_{a}^{T} \boldsymbol{u}_{a}$, where $\boldsymbol{u}_{a}$ are the components of $\vec{u}$ in $\mathcal{F}_{a}$.

The vector $\vec{u}$ can be expressed in multiple reference frames as such [46]

$$
\vec{u}=\overrightarrow{\mathcal{F}}_{a}^{T} \boldsymbol{u}_{a}=\overrightarrow{\mathcal{F}}_{b}^{T} \boldsymbol{u}_{b}
$$

where $\mathcal{F}_{b}$ is another reference frame. Taking the dot product of $\overrightarrow{\mathcal{F}}_{b}$ with both sides gives

$$
\overrightarrow{\mathcal{F}}_{b} \cdot \overrightarrow{\mathcal{F}}_{b}^{T} \boldsymbol{u}_{b}=\overrightarrow{\mathcal{F}}_{b} \cdot \overrightarrow{\mathcal{F}}_{a}^{T} \boldsymbol{u}_{a}
$$

which results in

$$
\boldsymbol{u}_{b}=\boldsymbol{C}_{b a} \boldsymbol{u}_{a}
$$

where $\boldsymbol{C}_{b a}$ is a rotation matrix that expresses the components of $\mathcal{F}_{a}$ in $\mathcal{F}_{b}$. This rotation matrix is expressed as

$$
\begin{aligned}
\boldsymbol{C}_{b a} & =\left[\begin{array}{l}
\vec{b}_{x} \\
\vec{b}_{y} \\
\vec{b}_{z}
\end{array}\right] \cdot\left[\begin{array}{lll}
\vec{a}_{x} & \vec{a}_{y} & \vec{a}_{z}
\end{array}\right] \\
& =\left[\begin{array}{lll}
\vec{b}_{x} \cdot \vec{a}_{x} & \vec{b}_{x} \cdot \vec{a}_{y} & \vec{b}_{x} \cdot \vec{a}_{z} \\
\vec{b}_{y} \cdot \vec{a}_{x} & \vec{b}_{y} \cdot \vec{a}_{y} & \vec{b}_{y} \cdot \vec{a}_{z} \\
\vec{b}_{z} \cdot \vec{a}_{x} & \vec{b}_{z} \cdot \vec{a}_{y} & \vec{b}_{z} \cdot \vec{a}_{z}
\end{array}\right]
\end{aligned}
$$


Rotation matrices are often referred to as Direction Cosine Matrix [46]. More information regarding these is provided in Sec. 2.3.1. A vector can also be expressed in three reference frames as such

$$
\vec{u}=\overrightarrow{\mathcal{F}}_{a}^{T} \boldsymbol{u}_{a}=\overrightarrow{\mathcal{F}}_{b}^{T} \boldsymbol{u}_{b}=\overrightarrow{\mathcal{F}}_{c}^{T} \boldsymbol{u}_{c}
$$

In component form $\boldsymbol{u}_{c}$ can be obtained from either reference frame $\mathcal{F}_{a}$ or $\mathcal{F}_{c}$ as such

$$
\boldsymbol{u}_{c}=\boldsymbol{C}_{c a} \boldsymbol{u}_{a}=\boldsymbol{C}_{c b} \boldsymbol{u}_{b}
$$

and where $\boldsymbol{u}_{b}$ can be expressed in terms of $\boldsymbol{u}_{a}$ as

$$
\boldsymbol{u}_{b}=\boldsymbol{C}_{b a} \boldsymbol{u}_{a}
$$

Combining Eqs. (2.5) and (2.6) yields

$$
\boldsymbol{u}_{c}=\boldsymbol{C}_{c b} \boldsymbol{C}_{b a} \boldsymbol{u}_{a}
$$

which means that $\boldsymbol{C}_{c a}$ can be written as

$$
\boldsymbol{C}_{c a}=\boldsymbol{C}_{c b} \boldsymbol{C}_{b a}
$$

The above equation indicates that the product of rotation matrices is also a rotation matrix and successive rotations can be formulated by multiplying rotation matrices in the reverse order, from right to left [46].

\section{Cross Product}

The cross product of two vectors in their component form is used extensively in this thesis. Vectors cross product is expressed as [46]

$$
\vec{u} \times \vec{v}=\overrightarrow{\mathcal{F}}_{a}^{T} \boldsymbol{u}_{a}^{\times} \boldsymbol{v}_{a}
$$

where $\boldsymbol{u}_{a}^{\times}$is a skew-symmetric matrix given by

$$
\boldsymbol{u}_{a}^{\times}=\left[\begin{array}{ccc}
0 & -u_{z, a} & u_{y, a} \\
u_{z, a} & 0 & -u_{x, a} \\
-u_{y, a} & u_{x, a} & 0
\end{array}\right]
$$

\section{Dot Product}

The dot product (also called scalar product) of two vectors is also used throughout this thesis. The dot product in terms of the components is given by [46]

$$
\vec{u} \cdot \vec{v}=\boldsymbol{u}_{a}^{T} \boldsymbol{v}_{a}
$$




\subsubsection{Angular Velocity and Derivative of a Vector}

As discussed earlier, a vector can be expressed in two reference frames as such

$$
\vec{u}=\overrightarrow{\mathcal{F}}_{a}^{T} \boldsymbol{u}_{a}=\overrightarrow{\mathcal{F}}_{b}^{T} \boldsymbol{u}_{b}
$$

Given these two reference frames, it is assumed that $\mathcal{F}_{b}$ rotates with respect to $\mathcal{F}_{a}$ with angular velocity $\vec{\omega}_{b a}$, where $\left|\vec{\omega}_{b a}\right|$ is the rate of rotation and $\vec{\omega}_{b a} /\left|\vec{\omega}_{b a}\right|$ is the instantaneous axis of rotation [46]. The three orthogonal axis of $\mathcal{F}_{b}$ denoted by $\left\{\vec{b}_{x}, \vec{b}_{y}, \vec{b}_{z}\right\}$ are fixed. The time derivative of these axes is perpendicular to the rotation rate vector as well as the fixed axes. Thus, the time derivatives of the axes of $\mathcal{F}_{b}$ with respect to $\mathcal{F}_{a}$ can be expressed as [46]

$$
\begin{aligned}
& \dot{\vec{b}}_{x}=\vec{\omega}_{b a} \times \vec{b}_{x} \\
& \dot{\vec{b}}_{y}=\vec{\omega}_{b a} \times \vec{b}_{y} \\
& \dot{\vec{b}}_{z}=\vec{\omega}_{b a} \times \vec{b}_{z}
\end{aligned}
$$

which can be rewritten compactly as

$$
\dot{\overrightarrow{\mathcal{F}}}_{b}^{T}=\vec{\omega}_{b a} \times \overrightarrow{\mathcal{F}}_{b}^{T}
$$

The time derivative of $\vec{u}$ as seen in inertial reference frame $\mathcal{F}_{a}$ is [46]

$$
\dot{\vec{u}}=\overrightarrow{\mathcal{F}}_{a}^{T} \dot{\boldsymbol{u}}_{a}
$$

and the time derivative of the vector as seen in the rotating reference $\mathcal{F}_{b}$, denoted by ${ }^{\circ}$, is expressed as [46]

$$
\stackrel{\circ}{\vec{u}}=\overrightarrow{\mathcal{F}}_{b}^{T} \dot{\boldsymbol{u}}_{b}
$$

Taking the time derivative of $\vec{u}$ in $\mathcal{F}_{b}$ using the chain rule yields

$$
\dot{\vec{u}}=\overrightarrow{\mathcal{F}}_{b}^{T} \dot{\boldsymbol{u}}_{b}+\dot{\overrightarrow{\mathcal{F}}}_{b}^{T} \boldsymbol{u}_{b}
$$

Making use of Eq. (2.18) gives

$$
\dot{\vec{u}}=\stackrel{\circ}{\vec{u}}+\dot{\overrightarrow{\mathcal{F}}}_{b}^{T} \boldsymbol{u}_{b}
$$

Substituting Eq. (2.16) into Eq. (2.20) results in

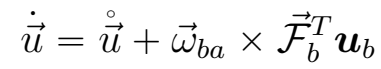

which can be simplified to

$$
\dot{\vec{u}}=\stackrel{\circ}{\vec{u}}+\vec{\omega}_{b a} \times \vec{u}
$$

The expression in Eq. (2.22) is referred to as the Transport Theorem and holds true for any vector [46]. 


\section{Velocity and Acceleration}

The position vector of a particle, denoted by $\vec{r}$, is from the origin of a reference frame to the particle. The rate of change of position is velocity, denoted by $\vec{v}$, and the rate of change of velocity is acceleration, denoted by $\vec{a}$. These three vectors are essential for developing equations of motion. This section highlights the expressions for the velocity and acceleration as seen from the inertial frame $\mathcal{F}_{a}$.

By using the Transport Theorem and applying it to the position vector $\vec{r}$, the velocity is obtained as such

$$
\vec{v}=\dot{\vec{r}}=\stackrel{\circ}{\vec{r}}+\vec{\omega}_{b a} \times \vec{r}
$$

Similarly, the acceleration is given by [46]

$$
\vec{a}=\dot{\vec{v}}=\ddot{\vec{r}}=\stackrel{\circ}{\vec{v}}+\vec{\omega}_{b a} \times \vec{v}
$$

where $\stackrel{\circ}{\vec{v}}$ can be obtained by applying the chain rule to Eq. (2.23) as such

$$
\stackrel{\circ}{\vec{v}}=\stackrel{\circ}{\vec{r}}+\stackrel{\circ}{\omega}_{b a} \times \vec{r}+\vec{\omega}_{b a} \times \stackrel{\circ}{\vec{r}}
$$

Substituting Eqs. (2.23) and (2.25) into Eq. (2.24) gives

$$
\begin{aligned}
\vec{a} & =\stackrel{\circ \vec{r}}{ }+\stackrel{\overrightarrow{\vec{\omega}}}{b a} \times \vec{r}+\vec{\omega}_{b a} \times \stackrel{\circ}{\vec{r}}+\vec{\omega}_{b a} \times\left(\stackrel{\circ}{\vec{r}}+\vec{\omega}_{b a} \times \vec{r}\right) \\
& =\stackrel{\circ}{\vec{r}}+\stackrel{\vec{\omega}}{b a}_{b a} \times \vec{r}+\vec{\omega}_{b a} \times \stackrel{\circ}{\vec{r}}+\vec{\omega}_{b a} \times \stackrel{\circ}{\vec{r}}+\vec{\omega}_{b a} \times \vec{\omega}_{b a} \times \vec{r}
\end{aligned}
$$

which can be simplified to [46]

$$
\vec{a}=\stackrel{\circ}{\vec{r}}+\stackrel{\circ}{\vec{\omega}}_{b a} \times \vec{r}+2 \vec{\omega}_{b a} \times \stackrel{\circ}{\vec{r}}+\vec{\omega}_{b a} \times\left(\vec{\omega}_{b a} \times \vec{r}\right)
$$

where,

$$
\begin{array}{ll}
\stackrel{\circ}{\vec{r}} & \sim \text { Acceleration as seen in } \mathcal{F}_{b} \\
\stackrel{\circ}{\vec{\omega}}_{b a} \times \vec{r} & \sim \text { Angular acceleration } \\
2 \vec{\omega}_{b a} \times \stackrel{\vec{r}}{r} & \sim \text { Coriolis acceleration } \\
\vec{\omega}_{b a} \times\left(\vec{\omega}_{b a} \times \vec{r}\right) & \sim \text { Centripetal acceleration }
\end{array}
$$




\subsubsection{Rigid Body Dynamics}

This section presents the equation that governs the rotational dynamical motion of a rigid body. The development of rotational dynamics requires the use of body fixed reference frame as well as Earth-centered inertial (ECI) reference frame, to be explained in Secs. 2.5.4 and 2.5.1, respectively. The body fixed reference frame is fixed to the spacecraft's body and it is located at the origin denoted by $O$. The rotation of the body fixed reference frame with respect to ECI reference frame is given by $\vec{\omega}=\overrightarrow{\mathcal{F}}_{B}^{T} \boldsymbol{\omega}_{B}$.

By definition, angular momentum is given by

$$
\vec{h}_{O} \triangleq \vec{r} \times \vec{p}
$$

where $\vec{p}$ is the linear momentum and can be expressed as

$$
\vec{p}=m \dot{\vec{r}}
$$

where $m$ is the mass and $\dot{\vec{r}}$ is the velocity [46].

Combining Eqs. (2.28) and (2.29) gives

$$
\vec{h}_{O}=m \vec{r} \times \dot{\vec{r}}
$$

Integrating over the entire volume, $\vec{h}_{O}$ becomes

$$
\vec{h}_{O}=\int_{V}(\vec{\rho} \times \dot{\vec{\rho}}) d m
$$

where $\vec{\rho}$ is the distance from the origin, $O$, to $d m$ as seen in Fig. 2.2 [46]. 


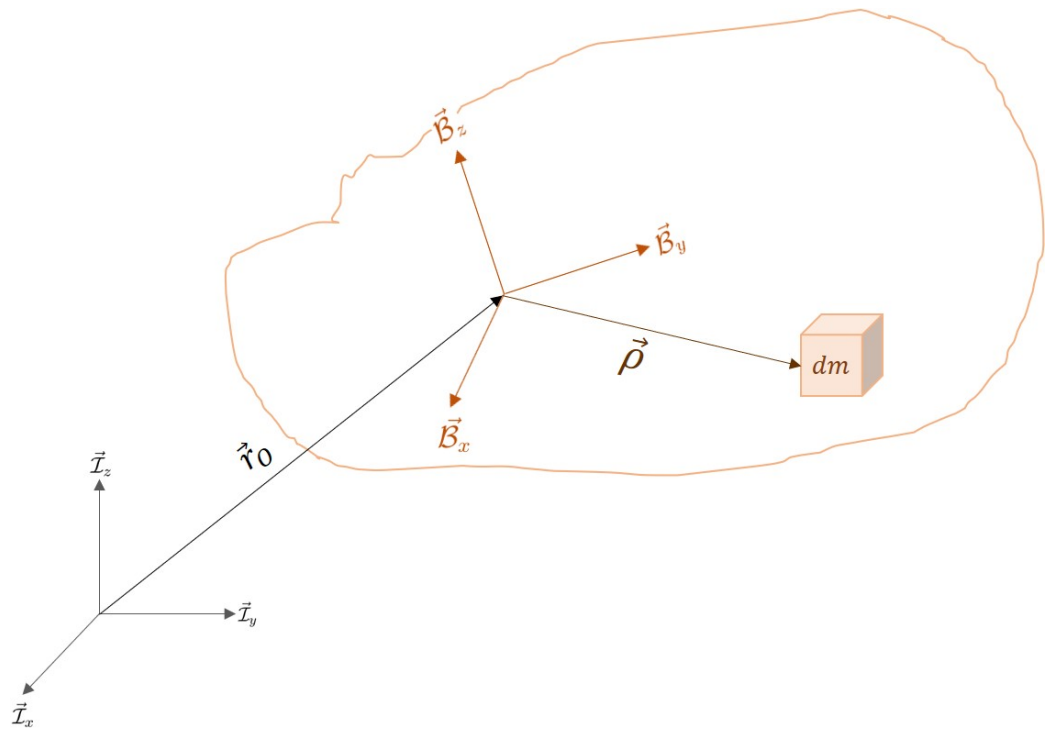

Figure 2.2: Dynamics of a Rigid Body

Making use of the Transport Theorem for $\dot{\vec{\rho}}$, Eq. (2.22), gives

$$
\vec{h}_{O}=\int_{V} \vec{\rho} \times(\stackrel{\grave{\rho}}{+} \vec{\omega} \times \vec{\rho}) d m
$$

Using the rigid body assumption, $\stackrel{\stackrel{\rho}{\rho}}{=} 0$ since all particles in the body are fixed and do not change with time [46]. This results in

$$
\begin{aligned}
\vec{h}_{O} & =\int_{V} \vec{\rho} \times(\vec{\omega} \times \vec{\rho}) d m \\
& =-\int_{V} \vec{\rho} \times(\vec{\rho} \times \vec{\omega}) d m
\end{aligned}
$$

where $\vec{\rho}=\overrightarrow{\mathcal{F}}_{B}^{T} \boldsymbol{\rho}_{B}, \vec{\omega}=\overrightarrow{\mathcal{F}}_{B}^{T} \boldsymbol{\omega}_{B}, \vec{h}_{O}=\overrightarrow{\mathcal{F}}_{B}^{T} \boldsymbol{h}_{O_{B}}$.

Equation (2.33) can be written in terms of its components as

$$
\boldsymbol{h}_{O_{B}}=\left(-\int_{V} \boldsymbol{\rho}_{B}^{\times} \boldsymbol{\rho}_{B}^{\times} d m\right) \boldsymbol{\omega}_{B}
$$

where the inertia matrix $\boldsymbol{J}$ is

$$
\boldsymbol{J} \triangleq-\int_{V} \boldsymbol{\rho}_{B}^{\times} \boldsymbol{\rho}_{B}^{\times} d m
$$

Thus Eq. (2.34) becomes

$$
\boldsymbol{h}_{O_{B}}=\boldsymbol{J} \boldsymbol{\omega}_{B}
$$


In the case where the point $O$ is located at the center of mass, the angular momentum equation becomes

$$
\boldsymbol{h}_{c}=\boldsymbol{J} \boldsymbol{\omega}_{B}
$$

By using the Transport Theorem, Eq. (2.22), the torque can be written as

$$
\vec{\tau}=\dot{\vec{h}}_{O}=\stackrel{\circ}{\vec{h}}+\vec{\omega} \times \vec{h}
$$

In the body fixed reference frame, Eq. (2.38) is expressed as

$$
\boldsymbol{\tau}_{c}=\dot{\boldsymbol{h}}_{c}+\boldsymbol{\omega}_{B}^{\times} \boldsymbol{h}_{c}
$$

where $\boldsymbol{h}_{c}$ and $\dot{\boldsymbol{h}}_{c}$ can be replaced by Eq. (2.37) and its time derivative as such

$$
\boldsymbol{\tau}_{c}=\boldsymbol{J} \dot{\boldsymbol{\omega}}_{B}+\boldsymbol{\omega}_{B}^{\times} \boldsymbol{J} \boldsymbol{\omega}_{B}
$$

Equation (2.40) is referred to as Euler's equation of motion, which describes the rotational dynamics of a rigid body subjected to external torques [46]. 


\subsection{Attitude Representation}

Attitude is the term used to describe the orientation of an object with respect to another one. Spacecraft attitude control is very important. For example, during a mission the solar panels have to be oriented towards the Sun for power generation. For communication satellites, antennas should be pointed towards the ground station to uplink and downlink data [47]. There are numerous ways for representing the attitude of a spacecraft. These are direction cosine matrix, Euler axis, and quaternions [48].

\subsubsection{Direction Cosine Matrix}

Direction Cosine Matrix (DCM) were previously explained in Sec. 2.2. One of the most importation class of rotations are those about one of the three coordinate axes: $\mathrm{x}, \mathrm{y}$, and $\mathrm{z}$. These three rotations are shown in Fig. 2.3, where a single rotation about one of the principle axis is performed to obtain $\mathcal{F}_{b}$ from $\mathcal{F}_{a}$.
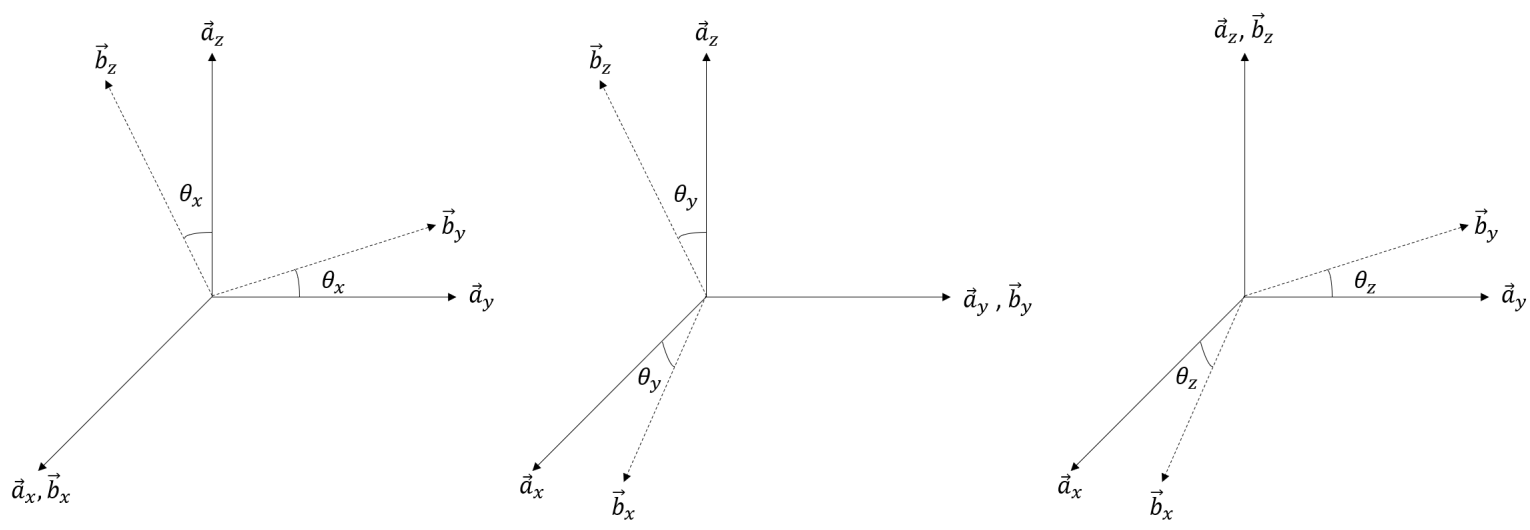

Figure 2.3: Principal Rotations

For a rotation about $\mathrm{x}$-axis, the matrix is [46]

$$
\boldsymbol{C}_{1}\left(\theta_{x}\right)=\left[\begin{array}{ccc}
1 & 0 & 0 \\
0 & \cos \theta_{x} & \sin \theta_{x} \\
0 & -\sin \theta_{x} & \cos \theta_{x}
\end{array}\right]
$$

Similarly, the rotation about y-axis and z-axis are given by

$$
\boldsymbol{C}_{2}\left(\theta_{y}\right)=\left[\begin{array}{ccc}
\cos \theta_{y} & 0 & -\sin \theta_{y} \\
0 & 1 & 0 \\
\sin \theta_{y} & 0 & \cos \theta_{y}
\end{array}\right]
$$


and

$$
\boldsymbol{C}_{3}\left(\theta_{z}\right)=\left[\begin{array}{ccc}
\cos \theta_{z} & \sin \theta_{z} & 0 \\
-\sin \theta_{z} & \cos \theta_{z} & 0 \\
0 & 0 & 1
\end{array}\right]
$$

As observed from the three matrices, each DCM requires 9 entries [47]. Often the orientation of a reference frame with another can be obtained by three successive principal rotations. There are 12 rotation sequences, each resulting in a different rotation matrix.

For example, let's consider a 3-2-1 rotation from $\mathcal{F}_{a}$ to $\mathcal{F}_{b}$. The first rotation about $\mathrm{z}$-axis results in an intermediate reference frame, $\mathcal{F}_{a}^{\prime}$, and the second rotation gives another intermediate reference frame, $\mathcal{F}_{a}^{\prime \prime}$. Finally, the last rotation, which is about the $\mathrm{x}$-axis results in $\mathcal{F}_{b}$. This $3-2-1$ rotation sequence is

$$
\begin{aligned}
& C_{3}\left(\theta_{z}\right): \mathcal{F}_{a}^{\prime} \leftarrow \mathcal{F}_{a} \\
& C_{2}\left(\theta_{y}\right): \mathcal{F}_{a}^{\prime \prime} \leftarrow \mathcal{F}_{a}^{\prime} \\
& C_{1}\left(\theta_{x}\right): \mathcal{F}_{b} \leftarrow \mathcal{F}_{a}^{\prime \prime}
\end{aligned}
$$

Therefore, the 3-2-1 rotation from $\mathcal{F}_{a}$ to $\mathcal{F}_{b}$ is given by

$$
\begin{aligned}
\boldsymbol{C}_{b a} & =\boldsymbol{C}_{1}\left(\theta_{x}\right) \boldsymbol{C}_{2}\left(\theta_{y}\right) \boldsymbol{C}_{3}\left(\theta_{z}\right) \\
& =\left[\begin{array}{ccc}
1 & 0 & 0 \\
0 & \cos \theta_{x} & \sin \theta_{x} \\
0 & -\sin \theta_{x} & \cos \theta_{x}
\end{array}\right]\left[\begin{array}{ccc}
\cos \theta_{y} & 0 & -\sin \theta_{y} \\
0 & 1 & 0 \\
\sin \theta_{y} & 0 & \cos \theta_{y}
\end{array}\right]\left[\begin{array}{ccc}
\cos \theta_{z} & \sin \theta_{z} & 0 \\
-\sin \theta_{z} & \cos \theta_{z} & 0 \\
0 & 0 & 1
\end{array}\right]
\end{aligned}
$$

\subsubsection{Euler Axis}

Another way of representing attitude is by a single rotation about a principle axis and an angle, denoted by $\vec{a}$ and $\phi$, respectively, where the angle is referred to as Euler angle [47]. This single rotation is shown in Fig. 2.4. The corresponding rotation matrix is given by [46]

$$
\boldsymbol{C}_{b a}=\cos \phi \boldsymbol{I}_{3}+(1-\cos \phi) \boldsymbol{a} \boldsymbol{a}^{T}-\sin \phi \boldsymbol{a}^{\times}
$$

where

$$
\boldsymbol{a} \equiv \boldsymbol{a}_{a} \equiv \boldsymbol{a}_{b}=\overrightarrow{\mathcal{F}}_{a} \cdot \vec{a}=\overrightarrow{\mathcal{F}}_{b} \cdot \vec{a}
$$

where $\vec{a}$ is given by

$$
\vec{a}=\left[\begin{array}{lll}
a_{x} & a_{y} & a_{z}
\end{array}\right]^{T}
$$




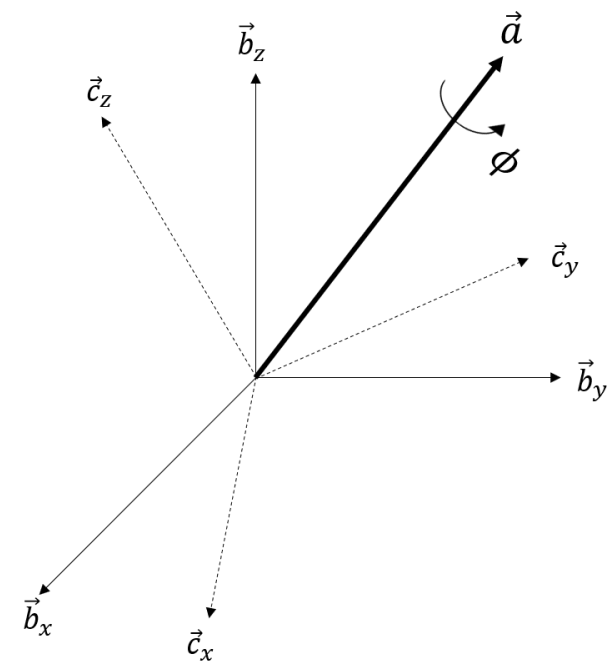

Figure 2.4: Euler Axis

An advantage of Euler-Axis is that it can be described by four parameters: three for the principle axis and one for the angle of rotation, compared to DCM, which requires 9 entries. Given a DCM, $\vec{a}$ and $\phi$ can be extracted [47].

\subsubsection{Quaternions}

The last method of describing attitude in this thesis is called quaternions, also referred to as Euler parameter set. Quaternions have some advantages over DCM and Euler axis. These include no singularities and no trigonometric functions in the kinematic differential equations [47]. Quaternions are given by [47]

$$
\boldsymbol{q}=\left[\begin{array}{c}
a_{x} \sin (\phi / 2) \\
a_{y} \sin (\phi / 2) \\
a_{z} \sin (\phi / 2) \\
\cos (\phi / 2)
\end{array}\right]=\left[\begin{array}{c}
\boldsymbol{\epsilon} \\
\eta
\end{array}\right]
$$

where $\boldsymbol{\epsilon}$ is the vector part of quaternions as such

$$
\boldsymbol{\epsilon}=\boldsymbol{a} \sin \left(\frac{\phi}{2}\right)
$$

and $\eta$ is the scalar part expressed as

$$
\eta=\cos \left(\frac{\phi}{2}\right)
$$


A rotation matrix can be calculated using quaternions. This relationship is expressed as [47]

$$
\begin{aligned}
\boldsymbol{C} & =\left(\eta^{2}-\boldsymbol{\epsilon}^{T} \boldsymbol{\epsilon}\right) \boldsymbol{I}+2 \boldsymbol{\epsilon} \boldsymbol{\epsilon}^{T}-2 \eta \boldsymbol{\epsilon}^{\times} \\
& =\left[\begin{array}{ccc}
q_{1}^{2}-q_{2}^{2}-q_{3}^{2}+q_{4}^{2} & 2\left(q_{1} q_{2}+q_{3} q_{4}\right) & 2\left(q_{1} q_{3}-q_{2} q_{4}\right) \\
2\left(q_{2} q_{1}-q_{3} q_{4}\right) & -q_{1}^{2}+q_{2}^{2}-q_{3}^{2}+q_{4}^{2} & 2\left(q_{2} q_{3}+q_{1} q_{4}\right) \\
2\left(q_{3} q_{1}+q_{2} q_{4}\right) & 2\left(q_{3} q_{2}-q_{1} q_{4}\right) & -q_{1}^{2}-q_{2}^{2}+q_{3}^{2}+q_{4}^{2}
\end{array}\right]
\end{aligned}
$$

The kinematic differential equation for quaternions is [49]

$$
\dot{\boldsymbol{q}}=\frac{1}{2}\left[\begin{array}{cc}
-\boldsymbol{\omega}_{b a}^{\times} & \boldsymbol{\omega}_{b a} \\
-\boldsymbol{\omega}_{b a}^{T} & 0
\end{array}\right] \boldsymbol{q}
$$

where $\boldsymbol{\omega}_{b a}$ is the angular rate.

Given a desired orientation in quaternion, $\boldsymbol{q}_{\text {des }}$, and the current one, $\boldsymbol{q}_{\text {det }}$, the quaternion error can be obtained as such [49]

$$
\begin{aligned}
\boldsymbol{q}_{e} & =\boldsymbol{q}_{\text {det }}{ }^{-1} \boldsymbol{q}_{\text {des }} \\
& =\left[\begin{array}{cc}
\eta_{\text {des }} \boldsymbol{I}-\boldsymbol{\epsilon}_{\text {des }}^{\times} & \boldsymbol{\epsilon}_{\text {des }} \\
\boldsymbol{\epsilon}_{\text {des }}^{T} & \eta_{\text {des }}
\end{array}\right]\left[\begin{array}{c}
\boldsymbol{\epsilon}_{\text {det }} \\
\eta_{\text {det }}
\end{array}\right]
\end{aligned}
$$

Quaternions are used for attitude representation throughout this thesis. Since quaternions rely on angular rate, the attitude calculation requires the use of Euler's equation of motion, Eq. (2.40). The attitude kinematics and dynamics block diagram is shown in Fig. 2.5, where $\boldsymbol{\tau}_{\text {applied }}$ comes from the control law and $\boldsymbol{\tau}_{\text {pert }}$ is the torque caused by external perturbations.

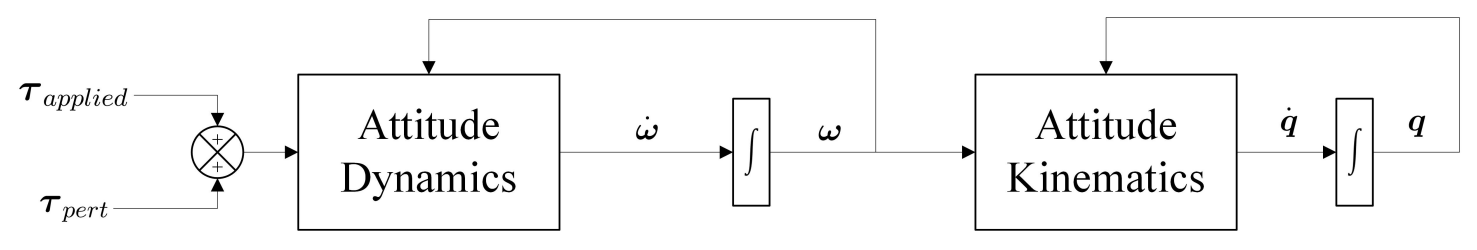

Figure 2.5: Attitude Kinematics and Dynamics 


\subsection{Two Body Problem}

This section introduces the Keplerian two body problem. The assumption is made that no external or internal forces are present except for gravity, that the gravitational bodies are spherical, and that the mass of the primary object is much larger than the orbiting body [45]. The Keplerian two body equation of motion is based on Newton's universal law of gravitation.

Newton's universal law of gravitation is used to describe the attraction between two objects. Newton's universal law states that two objects denoted by $M$ and $m$ are attracted to one another and the force of attraction is along the position vector connecting them. The force of attraction is proportional to the product of the two masses and inversely proportional to the square of the distance between them [48]. For this thesis, the attraction is between a spacecraft and the Earth. This fundamental equation is given by [50]

$$
\vec{F}_{12}=\frac{G M m \vec{r}}{r^{3}}
$$

where,

$G \sim$ Gravitational constant and equals $6.6726 \times 10^{-11} \mathrm{~m}^{3} / \mathrm{kg} . \mathrm{s}^{2}[51]$

$M \sim$ Mass of the celestial body [kg]

$m \sim$ Mass of the spacecraft $[\mathrm{kg}]$

$\vec{r} \sim$ Geocentric position vector of the spacecraft $[\mathrm{km}]$

Making use of Newton's second law, Eq. (2.54) can be rewritten as

$$
M \ddot{r}_{1}=\frac{G M m \vec{r}}{r^{3}}
$$

for the celestial body and

$$
m \ddot{r}_{2}=\frac{-G M m \vec{r}}{r^{3}}
$$

for the spacecraft.

Equations (2.55) and (2.56) can be simplified to

$$
\ddot{r}_{1}=\frac{G m \vec{r}}{r^{3}}
$$

and

$$
\ddot{r}_{2}=\frac{-G M \vec{r}}{r^{3}}
$$


The distance between the Earth and the spacecraft is shown graphically in Fig. 2.6. Mathematically, this is expressed as follows

$$
\vec{r}=\vec{r}_{2}-\vec{r}_{1}
$$

Taking the time derivative of the above twice yields

$$
\ddot{\vec{r}}=\ddot{\vec{r}}_{2}-\ddot{\vec{r}}_{1}
$$

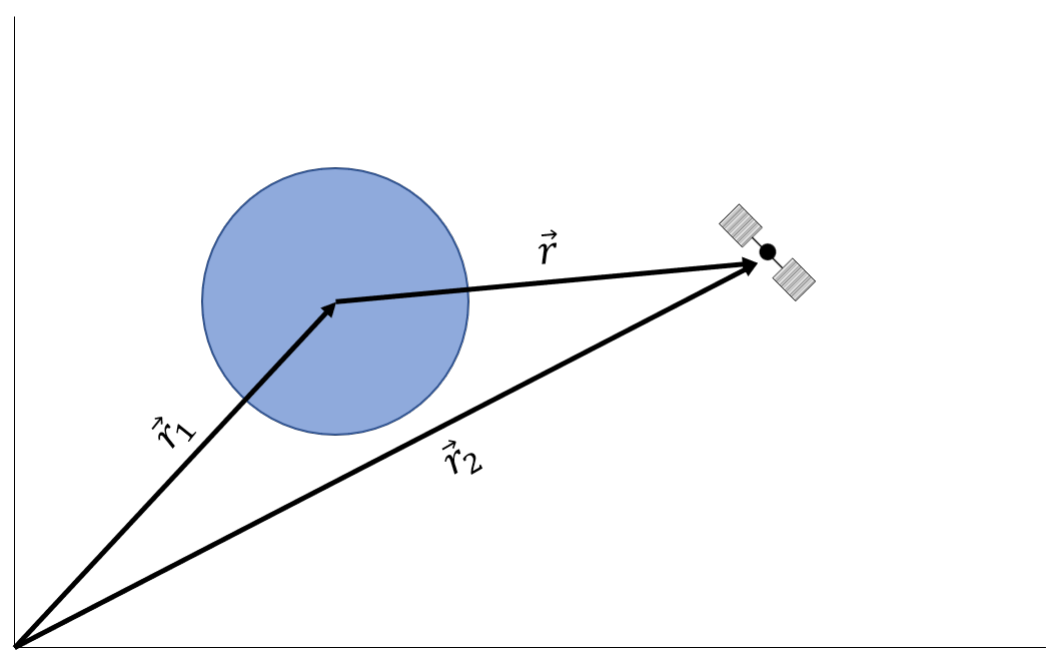

Figure 2.6: Two Body Diagram

Now substituting Eqs. (2.57) and (2.58) into Eq. (2.60) yields

$$
\ddot{\vec{r}}=-\frac{G M \vec{r}}{r^{3}}-\frac{G m \vec{r}}{r^{3}}
$$

The above equation can be simplified to

$$
\ddot{\vec{r}}=-\frac{G(M+m)}{r^{3}} \vec{r}
$$

The mass of the celestial body is much greater than the spacecraft, that is $M \gg m$. This results in

$$
\ddot{\vec{r}}=-\frac{G M}{r^{3}} \vec{r}
$$

The term $G M$ can be replaced by $\mu$, which is called the gravitational parameter. Thus Eq. (2.63) can be rewritten as

$$
\ddot{\vec{r}}=-\frac{\mu}{r^{3}} \vec{r}
$$

where $\mu$ is $398600.435436 \mathrm{~km}^{3} / \mathrm{s}^{2}$ for Earth ${ }^{1}$. Equation 2.64 is referred to as the two body problem equation of motion.

\footnotetext{
${ }^{1}$ From JPL HORIZONS on-line solar system data, https://ssd.jpl.nasa.gov/?horizons
} 


\subsection{Frames of Reference}

This section presents the various reference frames that are used in this thesis.

\subsubsection{Earth-Centered Inertial (ECI) Reference Frame}

ECI reference frame, denoted by $\mathcal{F}_{I}$. is used to define a spacecraft's position and velocity vectors with respect to Earth. The center of ECI reference frame is located at the center of Earth and it is fixed, meaning that this reference frame does not change with time.

The z-axis, denoted by $\overrightarrow{\mathcal{I}}_{z}$, is aligned with Earth's axis of rotation, $\overrightarrow{\mathcal{I}}_{x}$ is aligned with the vernal equinox, and $\overrightarrow{\mathcal{I}}_{y}$ completes the triad [45]. Vernal equinox defines the direction of the Sun on the first day of Spring as the Sun crosses the equator moving northward [45]. This reference frame is shown in Fig. 2.7.

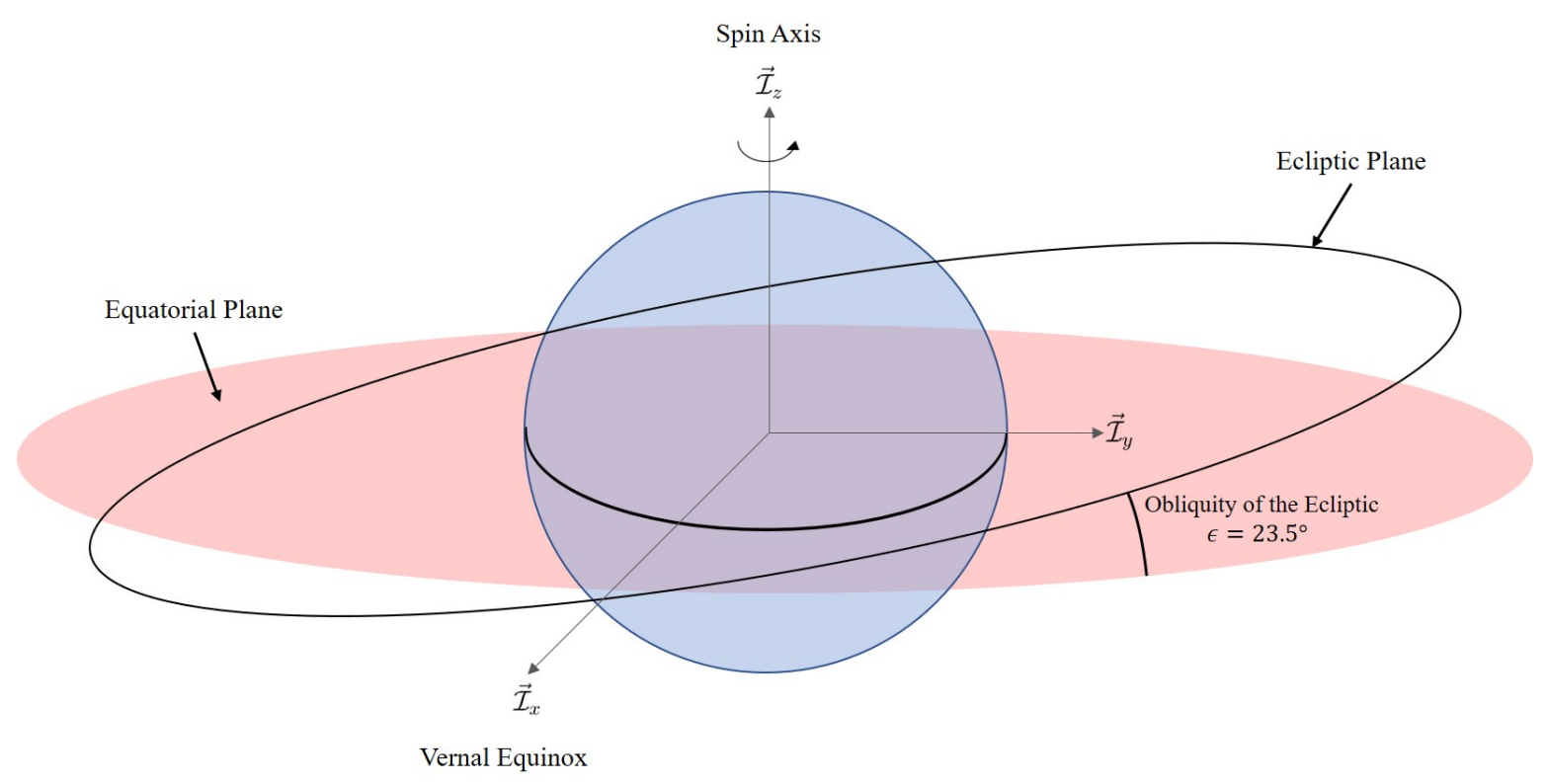

Figure 2.7: ECI Reference Frame 


\subsubsection{Perifocal Reference Frame}

Similar to ECI, the center of perifocal reference frame, $\mathcal{F}_{P}$, is located at the center of Earth. The x-axis denoted by $\overrightarrow{\mathcal{P}}_{x}$ is pointing towards the perigee of the orbit [45]. Perigee is the point on the orbit that is closest to the primary body, Earth in this case. The z-axis, denoted by $\overrightarrow{\mathcal{P}}_{z}$, is oriented perpendicular to the orbital plane, in the same direction as the angular momentum vector [45]. The y-axis, $\overrightarrow{\mathcal{P}}_{y}$, completes the triad. Perifocal reference frame is shown in Fig. 2.8 and is useful as it allows one to calculate the position and velocity vectors of a spacecraft in ECI reference frame given the orbital elements. This calculation is discussed in Sec. 2.6.1.

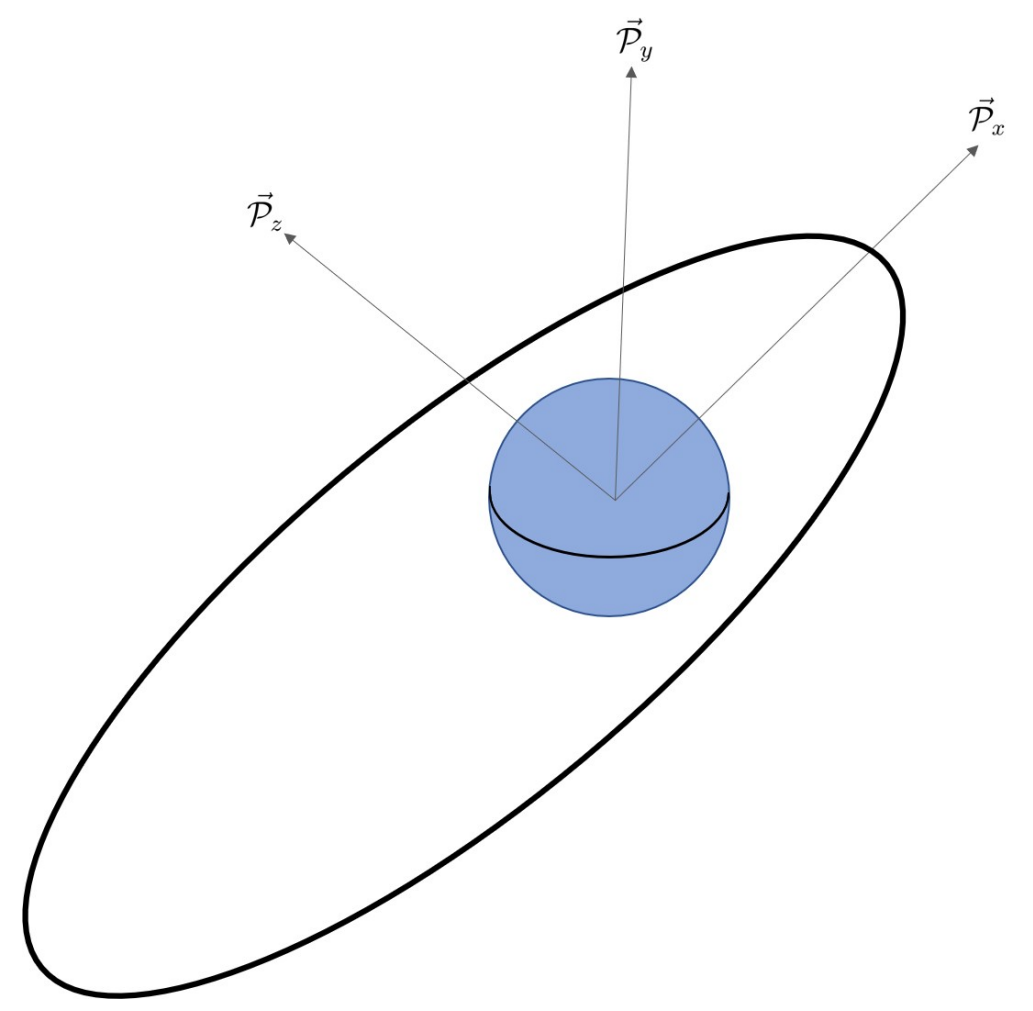

Figure 2.8: Perifocal Reference Frame 


\subsubsection{Local-Vertical Local-Horizontal (LVLH) Reference Frame}

LVLH reference frame is used for spacecraft formation flying and is illustrated in Fig. 2.9. The x component, denoted by $\overrightarrow{\mathcal{L}_{x}}$, also known as radial, points outwards from Earth towards the target spacecraft. The z component, cross-track, denoted by $\overrightarrow{\mathcal{L}_{z}}$ is orientated perpendicular to the orbital plane, and the along-track, $\overrightarrow{\mathcal{L}_{y}}$, completes the triad [45].

Given the position and velocity vectors of the target spacecraft, $\vec{r}_{t}$ and $\vec{v}_{t}$, respectively in ECI reference frame, the components of the LVLH reference frame can be calculated as such

$$
\begin{gathered}
\overrightarrow{\mathcal{L}}_{x}=\frac{\vec{r}_{t}}{\left|\vec{r}_{t}\right|} \\
\overrightarrow{\mathcal{L}}_{z}=\frac{\vec{r}_{t} \times \vec{v}_{t}}{\left|\vec{r}_{t} \times \vec{v}_{t}\right|} \\
\overrightarrow{\mathcal{L}}_{y}=\overrightarrow{\mathcal{L}}_{z} \times \overrightarrow{\mathcal{L}}_{x}
\end{gathered}
$$

Thus, the rotation matrix from ECI to LVLH is given by

$$
\boldsymbol{C}_{L I}=\left[\begin{array}{ccc}
\overrightarrow{\mathcal{L}}_{x}^{T} & \overrightarrow{\mathcal{L}}_{y}^{T} & \overrightarrow{\mathcal{L}}_{z}^{T}
\end{array}\right]^{T}
$$

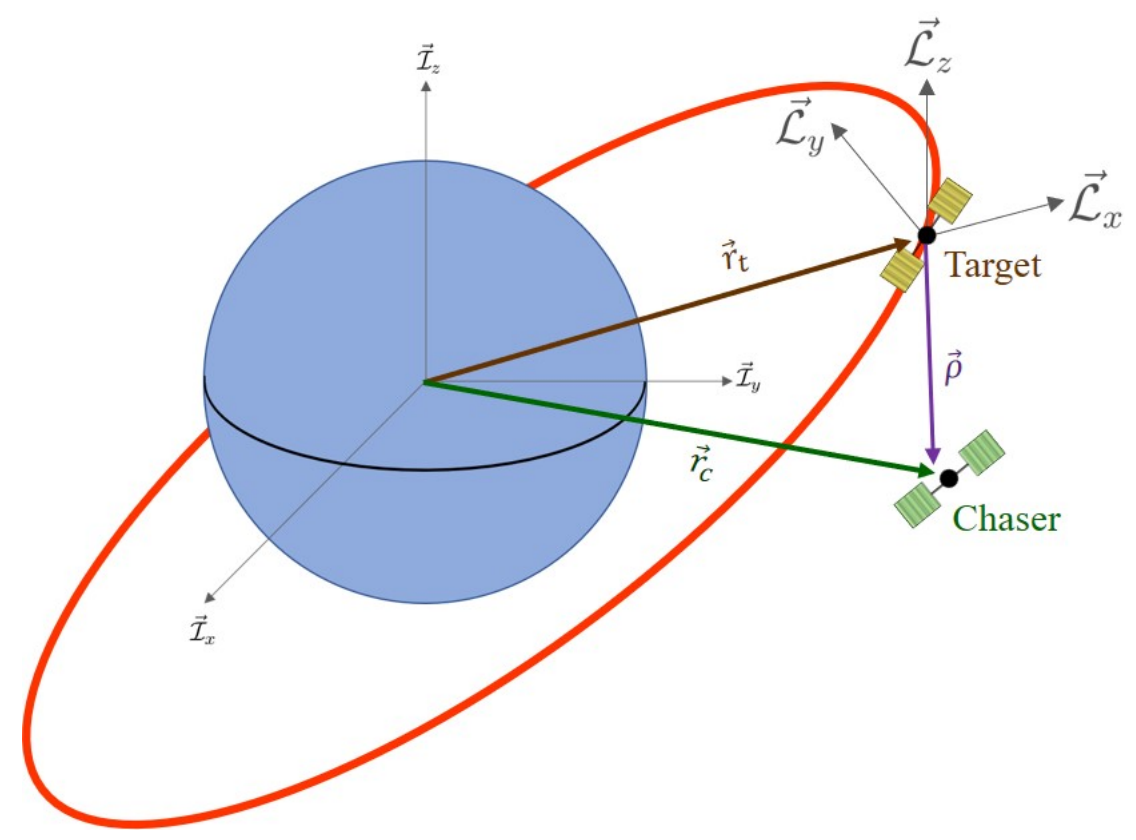

Figure 2.9: LVLH Reference Frame 


\subsubsection{Body Fixed Reference Frame}

Body fixed reference frame, denoted by $\mathcal{F}_{B}$, is fixed to the spacecraft and it is located at the center of the body. Often, it is aligned along the principal axes of inertia [47]. Body fixed reference frame is used for aligning the components within the spacecraft such as thrusters, sensors, and payload. The body fixed reference frame is used for attitude control for orienting the spacecraft relative to some other external reference frame [48].

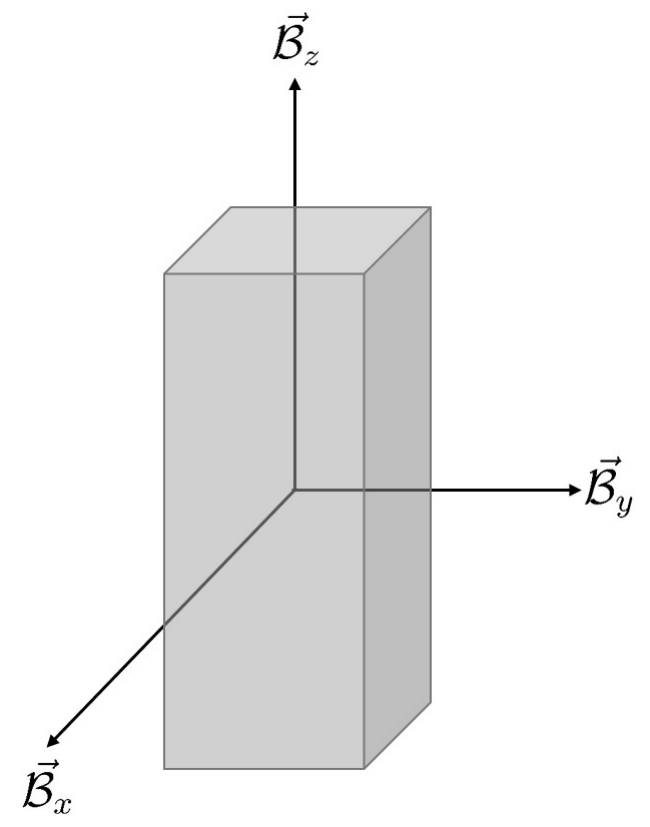

Figure 2.10: Body Fixed Reference Frame 


\subsection{Classical Orbital Elements}

Six orbital elements are used to describe the orientation and size of an orbit. These elements are divided into two groups; the first group describes the dimensional parameters of the orbit, which include size and shape. The second group describes the orientation of the orbital plane [51]. Five of these orbital elements are shown in Fig. 2.11.

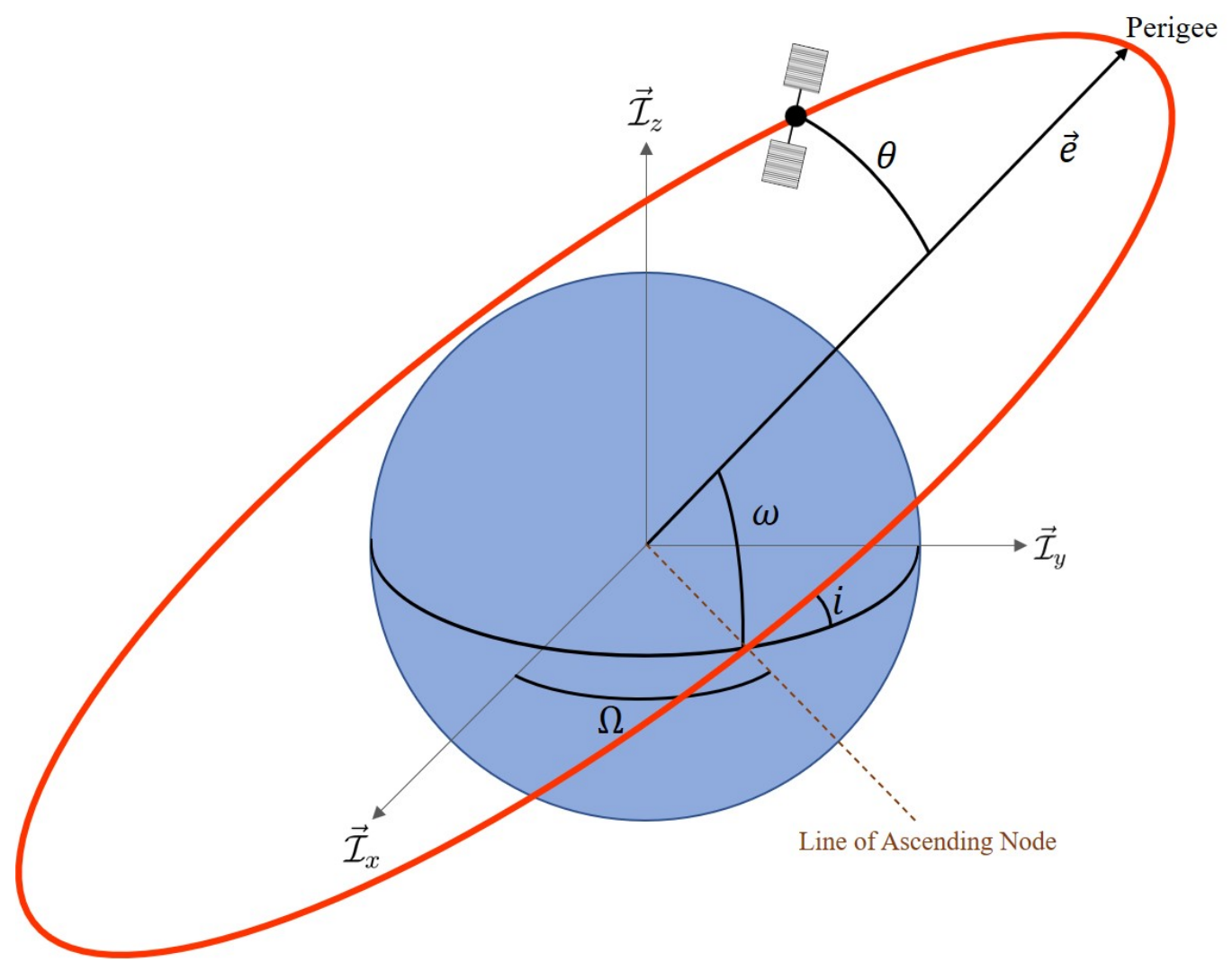

Figure 2.11: Orbital Elements

- $a$, semi major axis $[\mathrm{km}]$, describes the size of the orbital plane.

- e, eccentricity [unitless], describes the shape of the orbital plane. An eccentricity of zero denotes a circular orbit.

- $t_{p}$, time of perigee passage [s], or $\theta$, true anomaly [rad] relates the position of the spacecraft in orbit to time.

It is measured with respect to the perigee of the orbit.

- $i$, inclination [rad] describes the inclination of the orbital plane with respect to Earth's equatorial plane 
- $\Omega$, Right Ascension of the Ascending Node (RAAN) [rad], the angle between the vernal equinox to where the orbital plane crosses south to north at the equator.

The line caused by the intersection of the orbital plane and the equatorial plane is called the ascending node.

- $\omega$, argument of perigee $[\mathrm{rad}]$, the angle from the ascending node to the perigee of the orbit. 


\subsubsection{Position and Velocity in ECI}

This section provides the necessary equations to convert orbital elements to position and velocity vectors, $\vec{r}$ and $\vec{v}$, respectively. This calculation is needed to initialize the integration in the plant for the position and velocity vectors of the target and chaser spacecraft. First, the position and velocity vectors are calculated in the perifocal reference frame using the orbital elements. Then by using a rotation matrix, the components of $\vec{r}$ and $\vec{v}$ are transformed from $\mathcal{F}_{P}$ to their respective components in $\mathcal{F}_{I}$.

The position and velocity vector in perifocal reference frame are given by [45]

$$
\begin{aligned}
\vec{r} & =\overrightarrow{\mathcal{F}}_{p}^{T} \boldsymbol{r}_{p} \\
& =\overrightarrow{\mathcal{F}}_{p}^{T}\left[\begin{array}{c}
r \cos \theta \\
r \sin \theta \\
0
\end{array}\right] \\
\vec{v} & =\overrightarrow{\mathcal{F}}_{p}^{T} \boldsymbol{v}_{p} \\
& =\overrightarrow{\mathcal{F}}_{p}^{T}\left[\begin{array}{c}
-\sqrt{\frac{\mu}{p}} \sin \theta \\
-\sqrt{\frac{\mu}{p}}(e+\cos \theta) \\
0
\end{array}\right]
\end{aligned}
$$

where the magnitude of the position vector, $r$, can be calculated as such

$$
r=\frac{a\left(1-e^{2}\right)}{1+e \cos \theta}
$$

and the true anomaly, $\theta$, is expressed as

$$
\theta=2 \tan ^{-1}\left\{\frac{\sqrt{1+e}}{\sqrt{1-e}} \tan \frac{E}{2}\right\}
$$

The semilatus rectum, $p$, is obtained as

$$
p=a\left(1-e^{2}\right)
$$

In order to find the true anomaly, first the mean anomaly, $M$, needs to be calculated. The mean anomaly is given by [45]

$$
M=n\left(t-t_{p}\right)
$$


where $n$ is the mean orbital motion given by $n=\sqrt{\frac{\mu}{a^{3}}}$ and $t_{p}$ is the time of perigee passage.

The eccentric anomaly, $E$, is found using an iterative process, as follows [52]

$$
E_{i+1}=E_{i}-\frac{E_{i}-e \sin E_{i}-M}{1-e \cos E_{i}}
$$

where the first iteration, $E_{0}$ is set to equal the mean anomaly.

The components of $\vec{r}$ and $\vec{v}$ in ECI frame can be found as such

$$
\begin{aligned}
& \boldsymbol{r}_{I}=\boldsymbol{C}_{I P} \boldsymbol{r}_{P} \\
& \boldsymbol{v}_{I}=\boldsymbol{C}_{I P} \boldsymbol{v}_{P}
\end{aligned}
$$

where the rotation from perifocal reference frame to ECI reference frame is given by a 3-1-3 rotation with angles $\omega, i$, and $\Omega$, respectively, as such [52]

$$
\begin{aligned}
\boldsymbol{C}_{I P} & =\left[\begin{array}{ccc}
\cos \Omega & -\sin \Omega & 0 \\
\sin \Omega & \cos \Omega & 0 \\
0 & 0 & 1
\end{array}\right]\left[\begin{array}{ccc}
1 & 0 & 0 \\
0 & \cos i & -\sin i \\
0 & \sin i & \cos i
\end{array}\right]\left[\begin{array}{ccc}
\cos \omega & -\sin \omega & 0 \\
\sin \omega & \cos \omega & 0 \\
0 & 0 & 1
\end{array}\right] \\
& =\left[\begin{array}{ccc}
c_{\Omega} c_{\omega}-s_{\Omega} c_{i} s_{\omega} & -c_{\Omega} s_{\omega}-s_{\Omega} c_{i} c_{\omega} & s_{\Omega} s_{i} \\
s_{\Omega} c_{\omega}+c_{\Omega} c_{i} s_{\omega} & -s_{\Omega} s_{\omega}+c_{\Omega} c_{i} c_{\omega} & -c_{\Omega} s_{i} \\
s_{i} s_{\omega} & s_{i} c_{\omega} & c_{i}
\end{array}\right]
\end{aligned}
$$

where $c=\cos$ and $s=\sin$. 


\subsection{Perturbations}

This section discusses the various orbital and attitude perturbations that are used in the MATLAB/Simulink numerical simulator. This includes perturbations due to Earth's oblateness, third body, aerodynamic drag, and solar radiation pressure. All orbital perturbations are calculated in ECI reference frame, while attitude ones are calculated in body fixed reference frame.

\subsubsection{Gravity Perturbations due to Earth's Oblateness}

The two body equation of motion assumes that Earth is a point mass and that the total mass is at the center of Earth; however, the mass distribution of Earth is not symmetric and can perturb the motion of spacecraft in orbit [46]. The spacecraft force per unit mass is given by [46]

$$
\vec{f}=-\frac{\mu}{r^{3}} \vec{r}
$$

One can obtain a potential function for Eq. (2.79) as such

$$
\vec{f}=\vec{\nabla} U
$$

where $U$ can be expressed in a form of a series of Legendre polynomials as follows [46]

$$
\begin{aligned}
U=\frac{\mu}{r}[ & -\sum_{n=2}^{\infty} J_{n}\left(\frac{R_{\oplus}}{r}\right)^{n} P_{n} \sin \delta \\
& \left.+\sum_{n=2}^{\infty} \sum_{m=1}^{n}\left(\frac{R_{\oplus}}{r}\right)^{n} P_{n, m} \sin \delta\left(C_{n, m} \cos (m \lambda)+S_{n, m} \sin (m \lambda)\right)\right]
\end{aligned}
$$

where the coefficients $J_{n}, C_{n, m}$, and $S_{n, m}$ are obtained experimentally from satellite observations and $P_{n}$ is the associated Legendre function.

For rotationally symmetric bodies, $C_{n, m}=S_{n, m}=0$ [46]. Therefore, Eq. (2.81) can be simplified to

$$
U=-\frac{\mu}{r} \sum_{n=2}^{\infty} J_{n}\left(\frac{R_{\oplus}}{r}\right)^{n} P_{n} \sin \delta
$$

The most dominant perturbing effect for Earth is due to the term $J_{2}$. The perturbing potential due to $J_{2}$ is given by

$$
U=-\frac{\mu}{r^{3}} J_{2}\left(\frac{3}{2} \sin \delta-\frac{1}{2}\right)
$$


Making use of spherical coordinates definition, the perturbation acceleration due to $J_{2}$, with respect to the unit vectors of ECI reference frame, can be expressed as [46]

$$
\vec{a}_{J_{2}}=\frac{3 \mu_{\oplus} J_{2} R_{\oplus}^{2}}{2 r^{5}}\left\{\left[\frac{5\left(\vec{r} \cdot \overrightarrow{\mathcal{I}}_{x}\right)^{2}}{r^{2}}-1\right] \vec{r}-2\left(\vec{r} \cdot \overrightarrow{\mathcal{I}}_{z}\right) \overrightarrow{\mathcal{I}}_{z}\right\}
$$

where,

$$
\begin{aligned}
\mu_{\oplus} & \sim \text { Gravitational parameter of Earth }\left[\mathrm{km}^{3} / \mathrm{s}^{2}\right] \\
J_{2} & \sim \text { Second zonal harmonics coefficient [unitless] } \\
R_{\oplus} & \sim \text { Geocentric radius of Earth [km] } \\
\vec{r} & \sim \text { Position vector of spacecraft [km] } \\
\vec{I}_{x} & \sim \text { Unit vector defining ECI x-axis [unitless] } \\
\vec{I}_{z} & \sim \text { Unit vector defining ECI z-axis [unitless] }
\end{aligned}
$$

The components of $J_{2}$ acceleration in ECI reference frame are given by [45]

$$
\begin{aligned}
& a_{J_{2 x}}=\frac{\mu_{\oplus} X}{r^{3}}\left(\frac{3}{2} J_{2}\right)\left(\frac{R_{\oplus}}{r}\right)^{2}\left(5 \frac{Z^{2}}{r^{2}}-1\right) \\
& a_{J_{2 y}}=\frac{\mu_{\oplus} Y}{r^{3}}\left(\frac{3}{2} J_{2}\right)\left(\frac{R_{\oplus}}{r}\right)^{2}\left(5 \frac{Z^{2}}{r^{2}}-1\right) \\
& a_{J_{2 z}}=\frac{\mu_{\oplus} Z}{r^{3}}\left(\frac{3}{2} J_{2}\right)\left(\frac{R_{\oplus}}{r}\right)^{2}\left(5 \frac{Z^{2}}{r^{2}}-3\right)
\end{aligned}
$$

where $X, Y$, and $Z$ are the components of the position vector $\vec{r}$, and $r$ is the norm of the position vector. The numerical value of $J_{2}$ is $1.08262545 \times 10^{-3}$ [53]. 


\subsubsection{Third Body Perturbations}

The gravitational pull from other planets in the solar system can perturb the motion of spacecraft that are orbiting Earth. In this section, the Sun and the Moon are considered for third body perturbations. The graphical representation of third body perturbation due to the Sun is shown in Fig. 2.12. Mathematically, third body perturbations can be calculated as such [54]

$$
\overrightarrow{a_{3 B i}}=\mu_{i}\left(\frac{\overrightarrow{r_{i}}-\vec{r}}{\left|\overrightarrow{r_{i}}-\vec{r}\right|^{3}}-\frac{\vec{r}_{i}}{\vec{r}_{i}^{3}}\right)
$$

where,

$\mu_{i} \sim$ Gravitational parameter of the third body $\left[\mathrm{km}^{3} / \mathrm{s}^{2}\right]$

$\vec{r}_{i} \sim$ Geocentric position vector of the third body $[\mathrm{km}]$

$\vec{r} \sim$ Geocentric position vector of the spacecraft $[\mathrm{km}]$

The gravitational parameter for the Sun and the Moon are $\mu_{\odot}=132712440041.93938 \mathrm{~km}^{3} / \mathrm{s}^{2}$ and $\mu_{\S}=4902.800066 \mathrm{~km}^{3} / \mathrm{s}^{2}$, respectively ${ }^{2}$.

In order to find the position of the planets, one needs to determine the time. The algorithm used for this is called Julian date (JD).

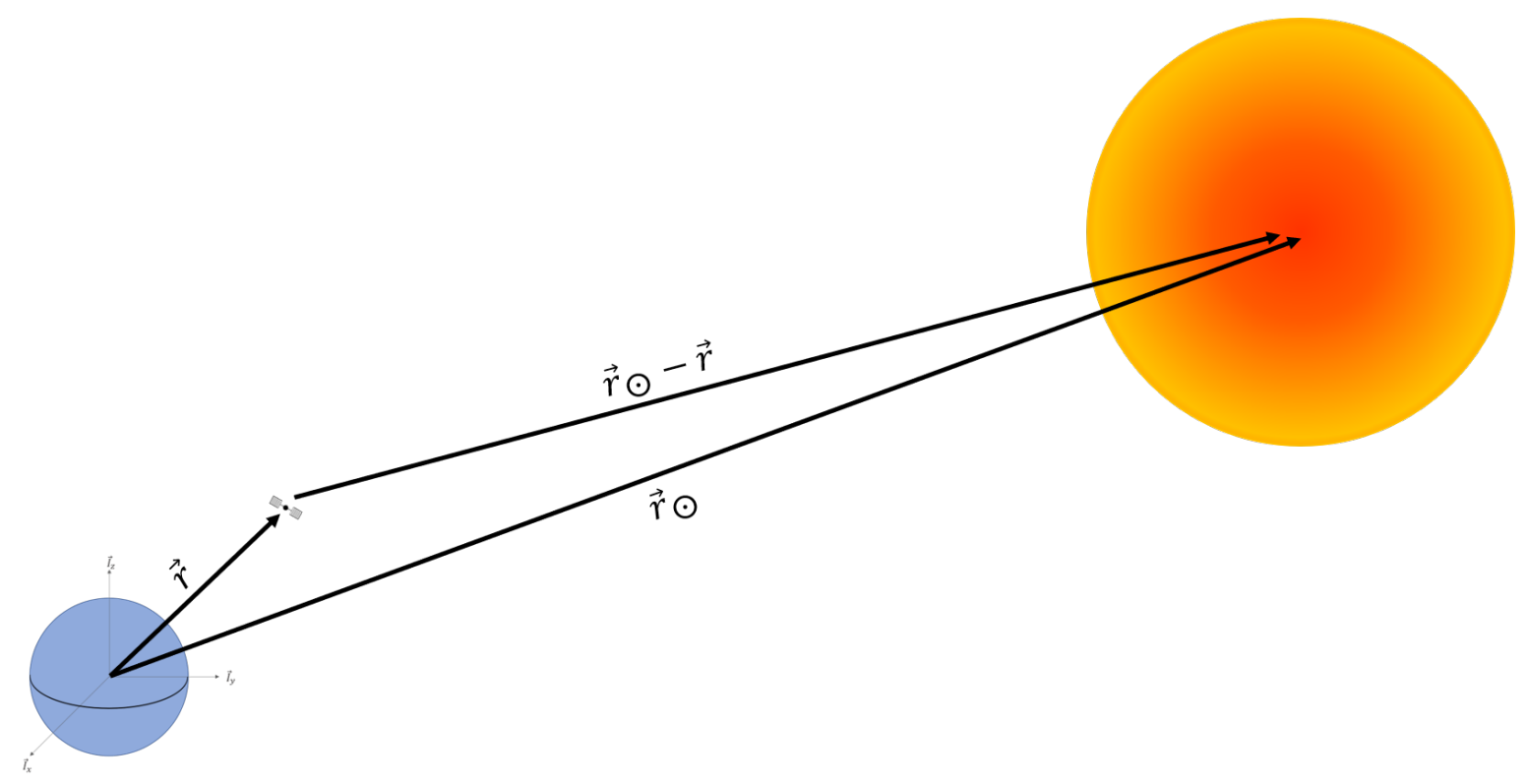

Figure 2.12: Geometrical Representation of a Spacecraft affected by Third Body Perturbation, using the Sun as an example

\footnotetext{
${ }^{2}$ From JPL HORIZONS on-line solar system data, https://ssd.jpl.nasa.gov/?horizons
} 


\section{Julian Date}

The JD algorithm is used for determining the sidereal time. Sidereal time is measured based on Earth's rotation with respect to fixed starts and it is 23 hours and 56 minutes [52].

A universal time coordinate (UTC) has been established, which is determined by the Sun's passage across Greenwich meridian. JD determines the number of days since noon UTC on January 1, 4713 BC [52]. The initial epoch is in UTC system and has the conventional Y : M : D : HH : MM : SS format. The number of days elapsed between January 1, 4713 BC at 12:00 UTC and the initial epoch can be calculated as such [55]

$$
J D_{0}=J D N_{0}+F_{o}
$$

where $F_{0}$ is

$$
F_{0}=\frac{H H-12}{24}+\frac{M M}{1440}+\frac{S S}{86400}
$$

and $J D N_{0}$ is

$$
\begin{aligned}
J D N_{0}=D-32075 & -\operatorname{int}\left[\frac{1461(Y+4800+C)}{4}\right] \\
& +\operatorname{int}\left[\frac{367(M-2-12 C)}{12}\right] \\
& -\operatorname{int}\left\{0.75 \operatorname{int}\left[\frac{Y+4900+C}{100}\right]\right\}
\end{aligned}
$$

where $C$ is

$$
C=\operatorname{int}\left[\frac{M-14}{12}\right]
$$

Julian centuries since J2000, $T_{0}$, is given by [55]

$$
T_{0}=\frac{J D-2451545}{36525}
$$




\section{Earth-Sun Distance}

Once the Julian date is determined, the distance from the Earth to the Sun can be calculated. First the mean longitude, $\lambda_{\odot}$, and the mean anomaly, $M_{\odot}$, of the Sun and obliquity of the ecliptic, $\epsilon$, are calculated as follows [48]

$$
\begin{aligned}
\lambda_{\odot} & =280+36000.771 T_{0} & & \left(0^{\circ} \leqslant \lambda_{\odot} \leqslant 360^{\circ}\right) \\
M_{\odot} & =357.5277233+35999.05034 T_{0} & & \left(0^{\circ} \leqslant M_{\odot} \leqslant 360^{\circ}\right) \\
\epsilon & =23.439291-0.0130042 T_{0} & &
\end{aligned}
$$

Given $\lambda_{\odot}$ and $M_{\odot}$, the ecliptic longitude is calculated as such

$$
\lambda_{e}=\lambda_{\odot}+1.914666471 \sin M_{\odot}+0.019994643 \sin \left(2 M_{\odot}\right)
$$

The distance from the Earth to the Sun, in AU, can be calculated according to [48]

$$
r_{\odot}=1.000140612+1.016708617 \cos M_{\odot}+0.000139589 \cos \left(2 M_{\odot}\right)
$$

Finally, the Earth-Sun vector components in ECI reference frame are given as follows [48]

$$
\begin{gathered}
\vec{r}_{\odot}=\overrightarrow{\mathcal{F}}_{I}^{T} \boldsymbol{r}_{\odot_{I}} \\
\boldsymbol{r}_{\odot_{I}}=r_{\odot}\left[\begin{array}{c}
\cos \lambda_{e} \\
\cos \epsilon \sin \lambda_{e} \\
\sin \epsilon \sin \lambda_{e}
\end{array}\right]
\end{gathered}
$$

\section{Earth-Moon Distance}

This section presents Earth-Moon distance calculation. The lunar ecliptic longitude, $\lambda_{\mathbb{}}$, lunar ecliptic latitude, $\delta_{\mathbb{S}}$, and lunar horizontal parallax, $H P_{\mathbb{S}}$, are given by [54]

$$
\begin{array}{rlrl}
\lambda_{\mathbb{}} & =b_{0}+c_{0} T_{0}+\sum_{i=1}^{6} a_{i} \sin \left(b_{i}+c_{i} T_{0}\right) & \left(0^{\circ} \leqslant \lambda_{\mathbb{}}<360^{\circ}\right) \\
\delta_{\mathbb{}} & =\sum_{i=1}^{4} d_{i} \sin \left(e_{i}+f_{i} T_{0}\right) & \left(0^{\circ} \leqslant \delta_{\mathbb{}}<360^{\circ}\right) \\
H P_{\mathbb{C}}=g_{0}+\sum_{i=1}^{4} g_{i} \cos \left(h_{i}+k_{i} T_{0}\right) & \left(0^{\circ} \leqslant H P_{\mathbb{C}}<180^{\circ}\right)
\end{array}
$$

where the coefficients $a_{i}, b_{i}, c_{i}, d_{i}, e_{i}, f_{i}, g_{i}, h_{i}$, and $k_{i}$ are provided in Table 2.1. 
Earth-Moon position vector components in ECI reference frame can then be calculated as such [54]

$$
\begin{aligned}
& \vec{r}_{\overparen{C}}=\overrightarrow{\mathcal{F}}_{I}^{T} \boldsymbol{r}_{\mathbb{C}_{I}}
\end{aligned}
$$

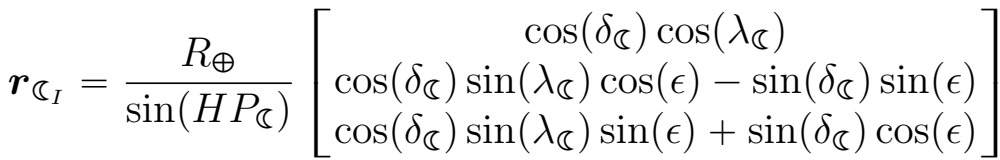

where $\epsilon$ was calculated previously in Eq. (2.96).

Table 2.1: Coefficients for Calculating the Position of the Moon [54]

\begin{tabular}{cccc}
\hline \multicolumn{4}{c}{ Longitude $\lambda_{\mathbb{}}$} \\
\hline $\mathrm{i}$ & $a_{i}$ & $b_{i}$ & $c_{i}$ \\
0 & - & 218.32 & $481,267.881$ \\
1 & 6.29 & 135.0 & $477,198.87$ \\
2 & -1.27 & 259.3 & $-413,335.36$ \\
3 & 0.66 & 235.7 & $890,534.22$ \\
4 & 0.21 & 269.9 & $954,397.74$ \\
5 & -0.19 & 357.5 & $35,999.05$ \\
6 & -0.11 & 106.5 & $966,404.03$ \\
\hline \multicolumn{4}{c}{ Latitude $\delta_{\mathbb{C}}$} \\
\hline $\mathrm{i}$ & $d_{i}$ & $e_{i}$ & $f_{i}$ \\
0 & - & - & - \\
1 & 5.13 & 93.3 & $483,202.03$ \\
2 & 0.28 & 220.2 & $960,400.89$ \\
3 & -0.28 & 318.3 & $6,003.15$ \\
4 & -0.17 & 217.6 & $-407,332.21$ \\
5 & - & - & - \\
6 & - & - & - \\
\hline \multicolumn{4}{c}{ Horizontal Parallax $H P_{\overparen{c}}$} \\
\hline $\mathrm{i}$ & $g_{i}$ & $h_{i}$ & $k_{i}$ \\
0 & 0.9508 & - & - \\
1 & 0.0518 & 135.0 & $477,198.87$ \\
2 & 0.0095 & 259.3 & $-413,355.38$ \\
3 & 0.0078 & 253.7 & $890,534.22$ \\
4 & 0.0028 & 269.9 & $954,397.70$ \\
5 & - & - & - \\
6 & - & - & - \\
& \multicolumn{3}{c}{-}
\end{tabular}




\subsubsection{Aerodynamic Drag}

Aerodynamic drag is the largest non-gravitational perturbation in low Earth orbit. The aerodynamic drag force acts in the opposite direction of the spacecraft's velocity. Aerodynamic drag perturbing force is expressed as [48]

$$
\vec{f}_{D}=-\frac{1}{2} \rho C_{D} A v_{r} \vec{v}_{r} \cos \alpha
$$

where,

$$
\begin{aligned}
\rho & \sim \text { Atmospheric density }\left[\mathrm{kg} / \mathrm{m}^{3}\right] \\
C_{D} \sim & \text { Drag coefficient [unitless] } \\
A \sim & \text { Surface area exposed to atmosphere }\left[\mathrm{m}^{2}\right] \\
\vec{v}_{r} \sim & \text { Spacecraft velocity relative to atmosphere }[\mathrm{m} / \mathrm{s}] \\
\cos \alpha \sim & \text { angle between } \vec{v}_{r} \text { and the surface area exposed } \\
& \text { to atmosphere [unitless] }
\end{aligned}
$$

More specifically, the angle $\alpha$ is obtained as

$$
\cos \alpha=\vec{n} \cdot \frac{\vec{v}_{r}}{\left|\vec{v}_{r}\right|}
$$

where $\vec{n}$ is the unit normal vector of the surface.

The drag coefficient has a range of 1.5 to 3.0 [56] and the average value of 2.25 is used in the simulation.

In terms of components, the drag force in ECI reference frame can be expressed as

$$
\vec{f}_{D}=\overrightarrow{\mathcal{F}}_{I} \boldsymbol{f}_{D_{I}}
$$

The drag force on the $\mathrm{k}$-th panel is given by

$$
\boldsymbol{f}_{D_{I}}^{k}=-\frac{1}{2} \rho C_{D} A\left\|\boldsymbol{v}_{r_{I}}\right\| \boldsymbol{v}_{r_{I}} \cos \alpha^{k}
$$

where $\cos \alpha^{k}$ can be obtained as such [48]

$$
\cos \alpha^{k}=\boldsymbol{n}_{B}^{k} \boldsymbol{C}_{B I} \frac{\boldsymbol{v}_{r_{I}}}{v_{r}}
$$

The net aerodynamic force is found by summing all the forces acting on each individual panel as follows

$$
\boldsymbol{f}_{D_{I}}=\sum_{i=1}^{n} \boldsymbol{f}_{D_{I}}^{k}
$$


The perturbing acceleration is then expressed as

$$
\vec{a}_{D}=\frac{\vec{f}_{D}}{m}
$$

where $m$ is the mass of the spacecraft.

The perturbing torque is obtained as

$$
\vec{\tau}_{D}=\vec{c}_{D} \times \vec{f}_{D}
$$

where $\vec{c}_{D}$ is the aerodynamic center of pressure.

In terms of its components, the torque in the body fixed reference frame for the k-th panel is

$$
\boldsymbol{\tau}_{D_{B}}^{k}=\boldsymbol{c}_{D_{B}}^{k}{ }^{\times} \boldsymbol{C}_{B I} \boldsymbol{f}_{D_{I}}^{k}
$$

The net aerodynamic torque can be obtained by summing the torques acting on each individual panel as such

$$
\boldsymbol{\tau}_{D}=\sum_{i=1}^{n} \boldsymbol{\tau}_{D}^{k}
$$

Spacecraft relative velocity $\vec{v}_{r}$ is given by

$$
\vec{v}_{r}=\vec{v}-\vec{\omega}_{\oplus} \times \vec{r}
$$

where $\vec{v}$ and $\vec{r}$ are the velocity and position vector of the spacecraft, respectively, and $\vec{\omega}_{\oplus}$ is Earth's rotation angular rate given by, in rad/s [56]

$$
\vec{\omega}_{\oplus}=\left[\begin{array}{c}
0 \\
0 \\
0.7292115 \times 10^{-4}
\end{array}\right]
$$

The density, $\rho$, can analytically be computed as follows [57]

$$
\rho=\rho_{0} \exp \left\{\frac{-\left(h-h_{0}\right)}{H}\right\}
$$

where,

$\rho_{0} \sim$ Reference density $\left[\mathrm{kg} / \mathrm{m}^{3}\right]$

$h \sim$ Geodetic altitude [km]

$h_{0} \sim$ Reference altitude $[\mathrm{km}]$

$H \sim$ Scale height $[\mathrm{km}]$ 
The values for $\rho_{0}, h_{0}$, and $H$ are given in Table 2.2.

Table 2.2: Exponential Atmospheric Model [57]

\begin{tabular}{|c|c|c|c|}
\hline $\begin{array}{c}\text { Altitude } \\
\qquad h[\mathrm{~km}]\end{array}$ & $\begin{array}{c}\text { Reference } \\
\text { Altitude } \\
h_{0}[\mathrm{~km}]\end{array}$ & $\begin{array}{c}\text { Reference } \\
\text { Density } \\
\rho_{0}\left[\mathrm{~kg} / \mathrm{m}^{3}\right]\end{array}$ & $\begin{array}{c}\text { Scale } \\
\text { Height } \\
\mathrm{H}[\mathrm{km}]\end{array}$ \\
\hline $0-25$ & 0 & 1.225 & 7.249 \\
\hline $25-30$ & 25 & $3.899 \times 10^{-2}$ & 6.349 \\
\hline $30-40$ & 30 & $1.744 \times 10^{-2}$ & 6.682 \\
\hline $40-50$ & 40 & $3.972 \times 10^{-3}$ & 7.554 \\
\hline $50-60$ & 50 & $1.057 \times 10^{-3}$ & 8.382 \\
\hline $60-70$ & 60 & $3.206 \times 10^{-4}$ & 7.714 \\
\hline $70-80$ & 70 & $8.770 \times 10^{-5}$ & 6.549 \\
\hline $80-90$ & 80 & $1.905 \times 10^{-5}$ & 5.799 \\
\hline $90-100$ & 90 & $3.396 \times 10^{-6}$ & 5.382 \\
\hline $100-110$ & 100 & $5.297 \times 10^{-7}$ & 5.877 \\
\hline $110-120$ & 110 & $9.661 \times 10^{-8}$ & 7.263 \\
\hline $120-130$ & 120 & $2.438 \times 10^{-8}$ & 9.473 \\
\hline $130-140$ & 130 & $8.484 \times 10^{-9}$ & 12.636 \\
\hline $140-150$ & 140 & $3.845 \times 10^{-9}$ & 16.149 \\
\hline $150-180$ & 150 & $2.070 \times 10^{-9}$ & 22.523 \\
\hline $180-200$ & 180 & $5.464 \times 10^{-10}$ & 29.470 \\
\hline $200-250$ & 200 & $2.789 \times 10^{-10}$ & 37.105 \\
\hline $250-300$ & 250 & $7.248 \times 10^{-11}$ & 45.546 \\
\hline $300-350$ & 300 & $2.418 \times 10^{-11}$ & 53.628 \\
\hline $350-400$ & 350 & $9.518 \times 10^{-12}$ & 53.298 \\
\hline $400-450$ & 400 & $3.725 \times 10^{-12}$ & 58.515 \\
\hline $450-500$ & 450 & $1.585 \times 10^{-12}$ & 60.828 \\
\hline $500-600$ & 500 & $6.967 \times 10^{-13}$ & 63.822 \\
\hline $600-700$ & 600 & $1.454 \times 10^{-13}$ & 71.835 \\
\hline $700-800$ & 700 & $3.614 \times 10^{-14}$ & 88.667 \\
\hline $800-900$ & 800 & $1.170 \times 10^{-14}$ & 124.64 \\
\hline $900-1000$ & 900 & $5.245 \times 10^{-15}$ & 181.05 \\
\hline 1000- & 1000 & $3.019 \times 10^{-15}$ & 268.00 \\
\hline
\end{tabular}




\subsubsection{Solar Radiation Pressure}

The Sun emits photons. The absorption and reflection of photons on the faces of a spacecraft introduces a force that can perturb the motion of the spacecraft. This perturbation is referred to as solar radiation pressure (SRP). The perturbing force due to SRP is given by [48]

$$
\overrightarrow{f_{S}}=-P_{\odot} C_{R} A \frac{\vec{r}_{\odot}-\vec{r}}{\left|\vec{r}_{\odot}-\vec{r}\right|} \cos \alpha
$$

where,

$$
\begin{aligned}
P_{\odot} & \sim \text { Solar radiation pressure }\left[\mathrm{Nm}^{-2}\right] \\
C_{R} & \sim \text { Radiation pressure coefficient [unitless] } \\
A & \sim \text { Surface area exposed to solar radiation }\left[\mathrm{m}^{2}\right] \\
\vec{r}_{\odot} & \sim \text { Geocentric position of the Sun }[\mathrm{m}] \\
\vec{r} & \sim \text { Geocentric position of the spacecraft }[\mathrm{m}] \\
\cos \alpha & \sim \text { angle between spacecraft-Sun unit vector and } \\
& \text { the surface area exposed to solar radiation [unitless] }
\end{aligned}
$$

More specifically, the angle is expressed as

$$
\cos \alpha=\vec{n} \cdot \vec{s}
$$

where $\vec{n}$ is the unit normal vector of the surface and

$$
\vec{s}=\frac{\vec{r}_{\odot}-\vec{r}}{\left|\vec{r}_{\odot}-\vec{r}\right|}
$$

The geocentric distance of the Sun, $\vec{r}_{\odot}$ has been previously calculated in Eq. (2.100).

The radiation pressure coefficient is 1.2 for solar panels and thus this value is chosen for the simulation. The value of $P_{\odot}$ is $4.56 \times 10^{-6} \mathrm{Nm}^{-2}$ [56].

The perturbing force acting on the k-th panel can be written in terms of its components in ECI as

$$
\boldsymbol{f}_{S_{I}}^{k}=-P_{\odot} C_{R} A^{k} \boldsymbol{s}_{I} \cos \alpha^{k}
$$

where $\cos \alpha^{k}$ can be obtained as such

$$
\cos \alpha^{k}=\boldsymbol{n}_{B}^{k} \boldsymbol{C}_{B I} \boldsymbol{s}_{I}
$$

The net SRP force is then found by

$$
\boldsymbol{f}_{S_{I}}=\sum_{i=1}^{n} \boldsymbol{f}_{S_{I}}^{k}
$$


The perturbing acceleration can then be calculated as such

$$
\vec{a}_{S}=\frac{\vec{f}_{S}}{m}
$$

where $m$ is the mass of the spacecraft.

The perturbing torque is obtained as

$$
\vec{\tau}_{S}=\vec{c}_{S} \times \vec{f}_{S}
$$

where $\vec{c}_{S}$ is the solar radiation center of pressure.

In terms of its components, the torque acting on the k-th panel in body fixed reference frame is

$$
\boldsymbol{\tau}_{S_{B}}^{k}=\boldsymbol{c}_{S_{B}}^{k}{ }^{\times} \boldsymbol{C}_{B I} \boldsymbol{f}_{S_{I}}
$$

The total torque is calculated as such

$$
\boldsymbol{\tau}_{S_{B}}=\sum_{i=1}^{n} \boldsymbol{\tau}_{S_{B}}^{k}
$$




\subsection{Spacecraft Formation Flying Dynamics}

This section describes the governing equations for SFF. These equations will be needed for the control design of the three sliding mode control laws, which are to be discussed in the next two chapters. These equations are expressed in LVLH reference frame. The chaser's position, as seen in Fig. 2.9, is expressed as such [45]

$$
\vec{r}_{c}=\vec{r}_{t}+\vec{\rho}
$$

where the vector $\vec{\rho}$, which is the relative position of the chaser spacecraft with respect to the target spacecraft is given by

$$
\vec{\rho}=\overrightarrow{\mathcal{F}}_{L}^{T} \boldsymbol{\rho}_{L}=\overrightarrow{\mathcal{F}}_{L}^{T}\left[\begin{array}{l}
x \\
y \\
z
\end{array}\right]
$$

Using the kinematics relations explained in Sec. 2.2, the velocity and acceleration of the chaser, $\dot{\vec{r}}_{c}$, and $\ddot{\vec{r}}$, respectively, are given by [45]

$$
\begin{gathered}
\dot{\vec{r}}_{c}=\stackrel{\circ}{\vec{r}}_{c}+\vec{\omega}_{L I} \times \vec{r}_{c} \\
\ddot{\vec{r}}_{c}=\stackrel{\circ}{\vec{r}}_{c}+\dot{\vec{\omega}}_{L I} \times \vec{r}_{c}+2 \vec{\omega}_{L I} \times \stackrel{\circ}{\vec{r}}_{c}+\vec{\omega}_{L I} \times\left(\vec{\omega}_{L I} \times \vec{r}_{c}\right)
\end{gathered}
$$

The vectors in these kinematic expressions can be expressed in the LVLH reference frame, $\mathcal{F}_{L}$, as

$$
\begin{aligned}
& \vec{r}_{c}=\overrightarrow{\mathcal{F}}_{L}^{T} \boldsymbol{r}_{c_{L}}=\overrightarrow{\mathcal{F}}_{L}^{T}\left[\begin{array}{c}
r_{t}+x \\
y \\
z
\end{array}\right] \\
& \stackrel{\circ}{r}_{c}=\overrightarrow{\mathcal{F}}_{L}^{T} \dot{\boldsymbol{r}}_{c_{L}}=\overrightarrow{\mathcal{F}}_{L}^{T}\left[\begin{array}{c}
\dot{r}_{t}+\dot{x} \\
\dot{y} \\
\dot{z}
\end{array}\right] \\
& \stackrel{\circ}{\vec{r}}_{c}=\overrightarrow{\mathcal{F}}_{L}^{T} \ddot{\boldsymbol{r}}_{c_{L}}=\overrightarrow{\mathcal{F}}_{L}^{T}\left[\begin{array}{c}
\ddot{r}_{t}+\ddot{x} \\
\ddot{y} \\
\ddot{z}
\end{array}\right] \\
& \vec{\omega}_{L I}=\overrightarrow{\mathcal{F}}_{L}^{T} \boldsymbol{\omega}_{L I}=\overrightarrow{\mathcal{F}}_{L}^{T}\left[\begin{array}{c}
0 \\
0 \\
\dot{\theta}
\end{array}\right] \\
& \dot{\vec{\omega}}_{L I}=\overrightarrow{\mathcal{F}}_{L}^{T} \dot{\boldsymbol{\omega}}_{L I}=\overrightarrow{\mathcal{F}}_{L}^{T}\left[\begin{array}{l}
0 \\
0 \\
\ddot{\theta}
\end{array}\right]
\end{aligned}
$$


Substituting Eqs. (2.133) to (2.137) into Eq. (2.132) yields

$$
\ddot{\vec{r}}_{c}=\overrightarrow{\mathcal{F}}_{L}^{T}\left[\begin{array}{c}
\ddot{r}_{t}+\ddot{x}-\ddot{\theta} y-2 \dot{\theta} \dot{y}-\dot{\theta}^{2}\left(r_{t}+x\right) \\
\ddot{y}+\ddot{\theta}\left(r_{t}+x\right)+2 \dot{\theta}\left(\dot{r}_{t}+\dot{x}\right)-\dot{\theta}^{2} y \\
\ddot{z}
\end{array}\right]
$$

Angular momentum, which can be related to the orbital position, is given by

$$
h=r_{t}^{2} \dot{\theta}
$$

Time differentiating the angular momentum equation yields

$$
\dot{h}=2 r_{t} \dot{r}_{t} \dot{\theta}+r_{t}^{2} \ddot{\theta}=0
$$

Assuming a Keplerian orbit, the angular momentum is conserved, meaning that it does not change with time [45]. Solving Eq. (2.140) for $\ddot{\theta}$ gives

$$
\ddot{\theta}=-2 \frac{\dot{r}_{t}}{r_{t}} \dot{\theta}
$$

The position vector for the target in LVLH is given by

$$
\vec{r}_{t}=\overrightarrow{\mathcal{F}}_{L}^{T} \boldsymbol{r}_{t_{L}}=\overrightarrow{\mathcal{F}}_{L}^{T}\left[\begin{array}{c}
r_{t} \\
0 \\
0
\end{array}\right]
$$

Time differentiating the position vector twice after some manipulation yields

$$
\ddot{\overrightarrow{r_{t}}}=\overrightarrow{\mathcal{F}}_{L}^{T}\left[\begin{array}{c}
\ddot{r}_{t}-\dot{\theta}^{2} r_{t} \\
0 \\
0
\end{array}\right]
$$

From two body equation of motion, the acceleration of the target in LVLH can be expressed as

$$
\ddot{\vec{r}_{t}}=-\overrightarrow{\mathcal{F}}_{L}^{T} \frac{\mu}{r_{t}^{3}}\left[\begin{array}{c}
r_{t} \\
0 \\
0
\end{array}\right]
$$

Equating Eqs. (2.143) and (2.144) gives

$$
\frac{\mu}{r_{t}^{3}}\left[\begin{array}{c}
r_{t} \\
0 \\
0
\end{array}\right]=\left[\begin{array}{c}
\ddot{r}_{t}-\dot{\theta}^{2} r_{t} \\
0 \\
0
\end{array}\right]
$$

which can be simplified and solved for $\ddot{r}_{t}$ as such

$$
\ddot{r}_{t}=\frac{-\mu}{r_{t}^{2}}+\dot{\theta}^{2} r_{t}
$$


Substituting Eqs. (2.141) and (2.146) into Eq. (2.138) gives

$$
\ddot{\vec{r}}_{c}=\overrightarrow{\mathcal{F}}_{L}^{T}\left[\begin{array}{c}
\ddot{x}-\dot{\theta}^{2} x-2 \dot{\theta}\left(\dot{y}-y \frac{\dot{r}_{t}}{r_{t}}\right)-\frac{\mu}{r_{t}^{2}} \\
\ddot{y}-\dot{\theta}^{2} y+2 \dot{\theta}\left(\dot{x}-x \frac{\dot{r}_{t}}{r_{t}}\right) \\
\ddot{z}
\end{array}\right]
$$

From the two body equations of motion, the chaser's acceleration can be expressed in the LVLH reference frame as such [45]

$$
\ddot{\vec{r}_{c}}=-\overrightarrow{\mathcal{F}}_{L}^{T} \frac{\mu}{r_{c}^{3}}\left[\begin{array}{c}
r_{t}+x \\
y \\
z
\end{array}\right]=\overrightarrow{\mathcal{F}}_{L}^{T}\left[\begin{array}{c}
a_{x} \\
a_{y} \\
a_{z}
\end{array}\right]
$$

Combining Eqs. (2.147) and (2.148) results in the exact nonlinear translational dynamics of the chaser with respect to the target in the LVLH reference frame [45]

$$
\begin{gathered}
\ddot{x}-\dot{\theta}^{2} x-2 \dot{\theta}\left(\dot{y}-y \frac{\dot{r}_{t}}{r_{t}}\right)-\frac{\mu}{r_{t}^{2}}=-\frac{\mu}{r_{c}^{3}}\left(r_{t}+x\right) \\
\ddot{y}-\dot{\theta}^{2} y+2 \dot{\theta}\left(\dot{x}-x \frac{\dot{r}_{t}}{r_{t}}\right)=-\frac{\mu}{r_{c}^{3}} y \\
\ddot{z}=-\frac{\mu}{r_{c}^{3}} z
\end{gathered}
$$

If the relative distance of the chaser and target spacecraft is small with respect to the orbital radius of the target spacecraft, $r_{t}$, Eqs. (2.149) to (2.151) can be further simplified to

$$
\begin{gathered}
\ddot{x}-x\left(\dot{\theta}^{2}+2 \frac{\mu}{r_{t}^{3}}\right)-2 \dot{\theta} \dot{y}-\ddot{\theta} y=0 \\
\ddot{y}-y\left(\dot{\theta}^{2}-\frac{\mu}{r_{t}^{3}}\right)+2 \dot{\theta} \dot{x}+\ddot{\theta} x=0 \\
\ddot{z}+\frac{\mu}{r_{t}^{3}} z=0
\end{gathered}
$$




\subsection{State Space Representation}

The dynamics equations of motion for SFF, given by Eqs. (2.149) to (2.151) in Sec. 2.8, can be formulated as a nonlinear state-space model given by

$$
\dot{\boldsymbol{x}}(t)=\boldsymbol{A}(t) \boldsymbol{x}(t)+\boldsymbol{H}(t)+\boldsymbol{B} \boldsymbol{u}(t)+\boldsymbol{a}_{p}
$$

with the states $\boldsymbol{x} \in \mathbb{R}^{6 \times 1}$, the control input $\boldsymbol{u} \in \mathbb{R}^{3 \times 1}$, and disturbance acceleration, perturbations, $\boldsymbol{a}_{\boldsymbol{d}} \in \mathbb{R}^{6 \times 1}$

$$
\begin{gathered}
\boldsymbol{x}=\left[\begin{array}{llllll}
x & y & z & \dot{x} & \dot{y} & \dot{z}
\end{array}\right]^{T} \\
\boldsymbol{u}=\left[\begin{array}{llll}
u_{x} & u_{y} & u_{z}
\end{array}\right]^{T} \\
\boldsymbol{a}_{p}=\left[\begin{array}{llllll}
0 & 0 & 0 & a_{p_{x}} & a_{p_{y}} & a_{p_{z}}
\end{array}\right]^{T}
\end{gathered}
$$

and the $\boldsymbol{A}(t), \boldsymbol{H}(t)$, and $\boldsymbol{B}$ matrices being

$$
\begin{aligned}
& \boldsymbol{A}(t)=\left[\begin{array}{cccccc}
0 & 0 & 0 & 1 & 0 & 0 \\
0 & 0 & 0 & 0 & 1 & 0 \\
0 & 0 & 0 & 0 & 0 & 1 \\
\dot{\theta}^{2}-\frac{\mu}{r_{c}^{3}} & \ddot{\theta} & 0 & 0 & 2 \dot{\theta} & 0 \\
-\ddot{\theta}^{c} & \dot{\theta}^{2}-\frac{\mu}{r_{c}^{3}} & 0 & -2 \dot{\theta} & 0 & 0 \\
0 & 0 & -\frac{\mu}{r_{c}^{3}} & 0 & 0 & 0
\end{array}\right]
\end{aligned}
$$

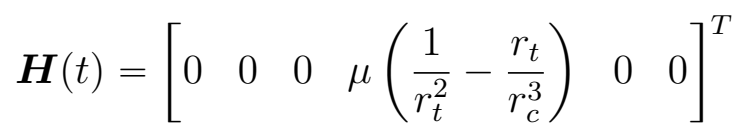

$$
\begin{aligned}
& \boldsymbol{B}=\left[\begin{array}{cccccc}
0 & 0 & 0 & \frac{1}{m_{c}} & 0 & 0 \\
0 & 0 & 0 & 0 & \frac{1}{m_{c}} & 0 \\
0 & 0 & 0 & 0 & 0 & \frac{1}{m_{c}}
\end{array}\right]^{T}
\end{aligned}
$$

and where $m_{c}$ is the mass of the chaser spacecraft. 


\subsection{Plant Overview}

Figure 2.13 shows the overall view of the plant that is implemented in MATLAB/Simulink. The plant includes perturbations and the two body equation of motion for both the chaser and the target. The two body equation of motion was described in Sec. 2.4 and perturbations were discussed in Sec. 2.7. The controller input will be discussed in Chapters 3 to 5. In Fig. 2.13, the subscript $t$ represents the target spacecraft and the subscript $c$ represents the chaser spacecraft.

By integrating the two body problem twice, the velocity and position vectors of the two spacecraft are calculated, which are then used to determine the LVLH reference frame and convert the relative position and velocity vectors between the two spacecraft from ECI to LVLH. The position and velocity vectors in LVLH are then used to calculate the error of the system and to determine the control input needed to maintain the desired trajectory.

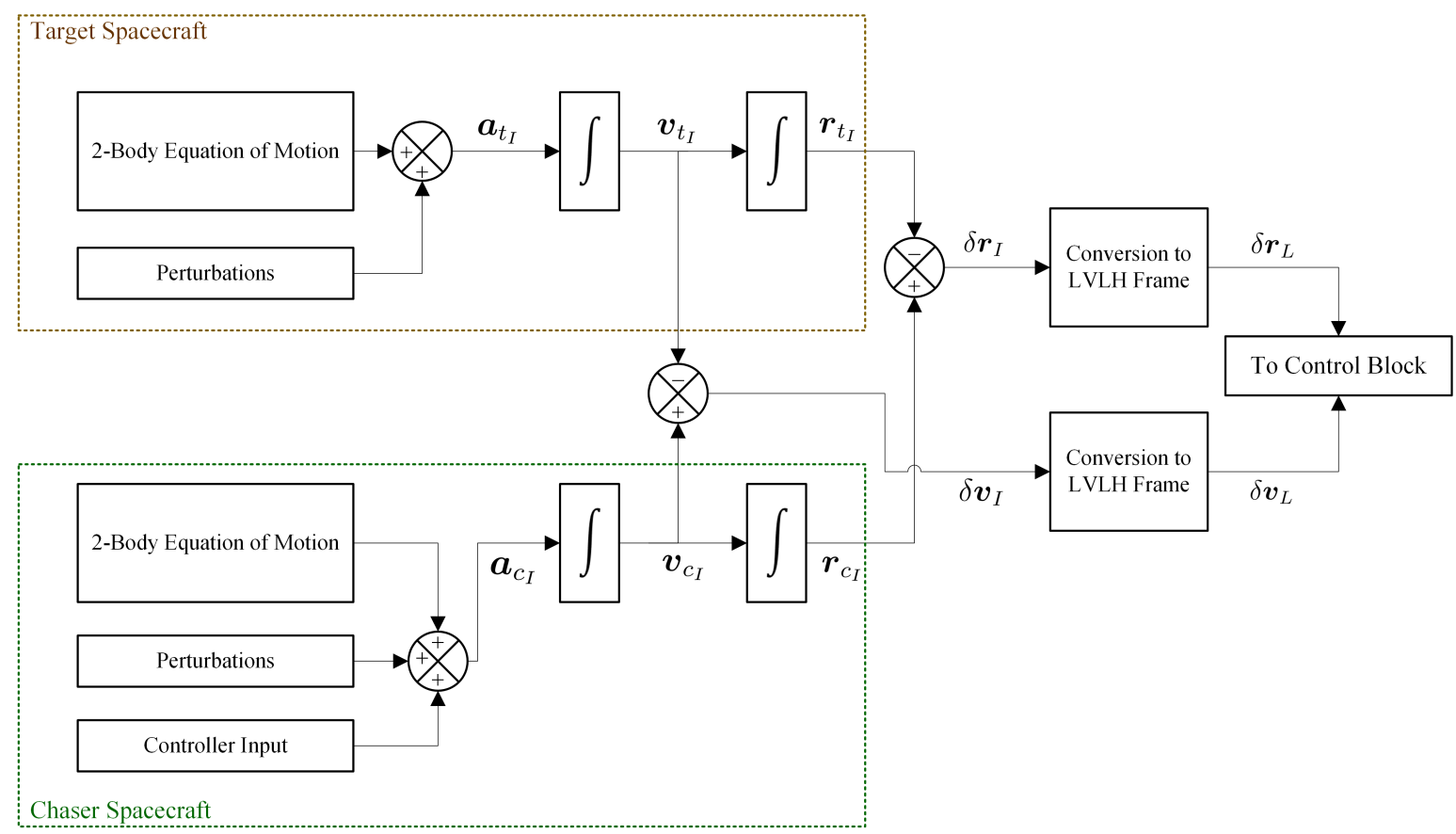

Figure 2.13: Plant Overview

The difference between the position vector of the chaser and target spacecraft is denoted by $\delta \vec{r}$ and the difference in their velocity vector is denoted by $\delta \vec{v}$. More 
specifically, $\delta \vec{r}$ and $\delta \vec{v}$ are calculated as such

$$
\begin{aligned}
\delta \vec{r} & =\vec{r}_{c}-\vec{r}_{t} \\
& =\overrightarrow{\mathcal{F}}_{I}^{T}\left(\boldsymbol{r}_{c_{I}}-\boldsymbol{r}_{t_{I}}\right) \\
& =\overrightarrow{\mathcal{F}}_{I}^{T}\left(\delta \boldsymbol{r}_{I}\right) \\
\delta \vec{v} & =\vec{v}_{c}-\vec{v}_{t} \\
& =\overrightarrow{\mathcal{F}}_{I}^{T}\left(\boldsymbol{v}_{c_{I}}-\boldsymbol{v}_{t_{I}}\right) \\
& =\overrightarrow{\mathcal{F}}_{I}^{T}\left(\delta \boldsymbol{v}_{I}\right)
\end{aligned}
$$

In LVLH reference frame, $\delta \boldsymbol{r}_{L}$ and $\delta \boldsymbol{v}_{L}$ are determined as such

$$
\begin{aligned}
\delta \boldsymbol{r}_{L} & =\boldsymbol{\rho} \\
& =\boldsymbol{C}_{L I} \delta \boldsymbol{r}_{I} \\
\delta \boldsymbol{v}_{L} & =\dot{\boldsymbol{\rho}} \\
& =\boldsymbol{C}_{L I}\left(\delta \boldsymbol{v}_{I}-\boldsymbol{\omega}_{L I} \times \delta \boldsymbol{r}_{I}\right)
\end{aligned}
$$

where $\boldsymbol{\omega}_{L I}$ is calculated as

$$
\boldsymbol{\omega}_{L I}=\frac{\boldsymbol{r}_{t} \times \boldsymbol{v}_{t}}{\left\|\boldsymbol{r}_{t}\right\|^{2}}
$$

and $\boldsymbol{C}_{L I}$ is given by

$$
\boldsymbol{C}_{L I}=\left[\begin{array}{ccc}
\overrightarrow{\mathcal{L}}_{x}^{T} & \overrightarrow{\mathcal{L}}_{y}^{T} & \overrightarrow{\mathcal{L}}_{z}^{T}
\end{array}\right]^{T}
$$

The components of the LVLH reference frame are calculated as such

$$
\begin{gathered}
\overrightarrow{\mathcal{L}}_{x}=\frac{\vec{r}_{t}}{\left|\overrightarrow{r_{t}}\right|} \\
\overrightarrow{\mathcal{L}_{z}}=\frac{\vec{r}_{t} \times \vec{v}_{t}}{\left|\vec{r}_{t} \times \vec{v}_{t}\right|} \\
\overrightarrow{\mathcal{L}}_{y}=\overrightarrow{\mathcal{L}}_{z} \times \overrightarrow{\mathcal{L}}_{x}
\end{gathered}
$$




\section{$2.11 \quad$ Fault Modelling}

Throughout the mission, actuators can experience different types of failure. These failures are explained in this section. The following cases relate the control input $u$, which comes from the control law to $u_{f}$, the control input after it has experienced a failure. This modified control input vector is then applied to the plant.

\section{Loss of Effectiveness}

Loss of effectiveness implies that the actuator's performance has degraded. This failure is mathematically expressed as $u_{f}=k u$, where $0<k<1$ [19,20,33]. The variable $k$ defines the extensiveness of actuator failure. For example, a value of 0.5 means that the actuator has lost its effectiveness by $50 \%$.

\section{Lock in Place Failure}

For this type of failure, the actuator is stuck at a certain value and fails to respond to the control input. This is given by $u_{f}=\bar{u}\left(t_{f}\right)$, where $\bar{u}$ is the value at which the actuator is stuck at. The time at which this failure happens is denoted by $t_{f}$, time of failure. [19,20,33].

\section{Float Type Failure}

In this scenario, the actuator has completely failed, meaning that it cannot produce any force or torque. The mathematical model for float type of failure is $u_{f}=0[19,20,33]$.

\section{Hard Over Failure}

Hard over failure denotes saturation. This means that the actuator has reached its maximum value and cannot produce more force or torque. This failure is expressed as $\left|u_{f}\right|=\left|u_{M}\right|$, where $u_{M}$ is the limit of the actuator, assuming that the lower and upper bounds are the same [33]. 


\section{Chapter 3}

\section{Nonsingular Terminal Sliding Mode Control}

This chapter discusses nonsingular terminal sliding mode (NTSMC) and modified NTSMC (MNTSMC). NTSMC and MNTSMC are the first two control law that will be used as a benchmark. Both of these control laws are part of the sliding mode control family (SMC). SMC is a robust nonlinear feedback that belongs to a type of variable structure control system. A more detailed description of SMC is given in Sec. 3.1: Introduction. Section 3.2: Tracking Error details the tracking error for NTSMC and Sec. 3.3: Control Law presents the equations for the sliding surface and the control law. Lyapunov stability analysis of NTSMC is discussed in Sec. 3.4: Stability Analysis. Due to the nature of the control law for sliding mode, the system experiences chattering, which is not favourable. Section 3.5: The Chattering Problem presents a method for eliminating the chattering issue. The modified NTSMC is presented in Sec. 3.6: Modified Nonsingular Terminal Sliding Mode Control. This is followed by Sec. 3.7: Finite Time Analysis, where the reaching and sliding time equations are given. 


\subsection{Introduction}

This section highlights the definition of SMC. In general, the objective of SMC is to bring the system on to the sliding surface. SMC consists of two phases: a reaching phase and a sliding phase. During the reaching phase, the system is driven such that it reaches the sliding surface denoted by $s=0$ in finite time. During the sliding phase, the trajectories remain on the sliding surface for all times [58]. The phase portrait of sliding mode is shown in Fig. 3.1.

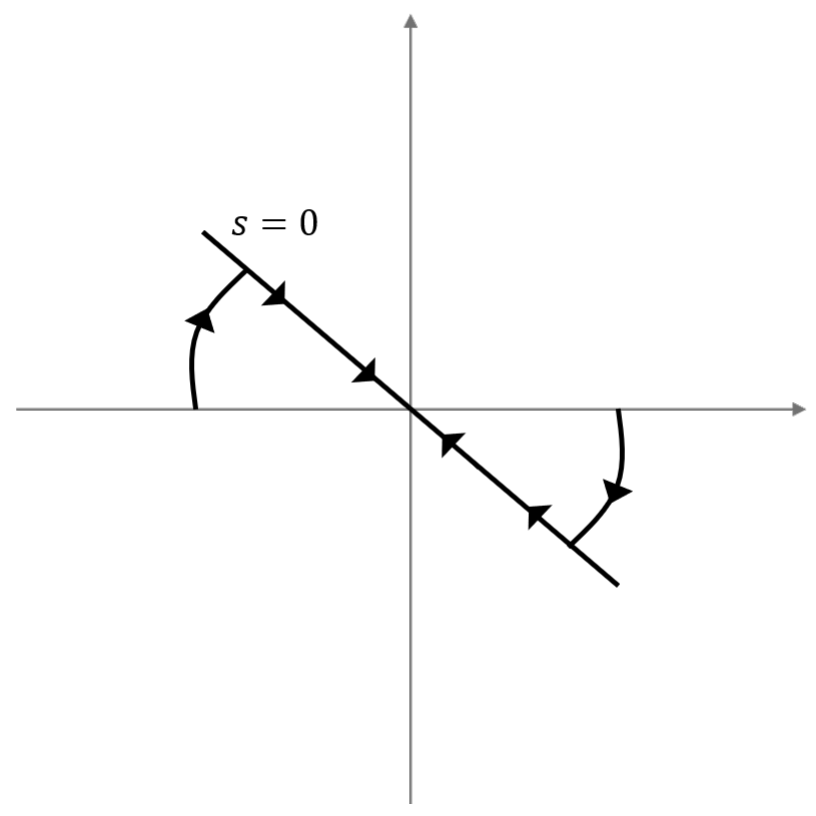

Figure 3.1: Phase Portrait of Sliding Mode control, adapted from [58]

Specifically, a NTSMC is discussed in this chapter, which is based on a control law that was developed by Gorard and Kumar [19]. NTSMC was first proposed by Feng et al. [59]. NTSMC prevents the singularity issue that occurs with a conventional terminal sliding mode control (TSM). TSM is based on the theory of finite time mechanism, which allows the system to have a faster convergence rate. This chapter also highlights a modified NTSMC, based on the work done by Cao et al. [20]. Cao et al. [20] modify the NTSMC law by alleviating the nonlinear switching surface. This modification to the switching surface causes the system to have a better performance compared to the conventional NTSMC. 


\subsection{Tracking Error}

For NTSMC, the tracking error is defined as [19]

$$
e=\rho-\rho_{d}
$$

where $\boldsymbol{\rho}_{d}$ and $\boldsymbol{\rho} \in \mathbb{R}^{3 \times 1}$ are the desired position and the actual position, respectively.

The derivative of the error is also required. The derivative of the error is given by

$$
\dot{\boldsymbol{e}}=\dot{\boldsymbol{\rho}}-\dot{\boldsymbol{\rho}}_{d}
$$

where $\dot{\boldsymbol{\rho}}_{d}$ is the derivative of $\boldsymbol{\rho}_{d}$ and $\dot{\boldsymbol{\rho}}$ is the derivative of $\boldsymbol{\rho}$.

\subsection{Control Law}

The overall block diagram for NTSMC is illustrated in Fig. 3.2.

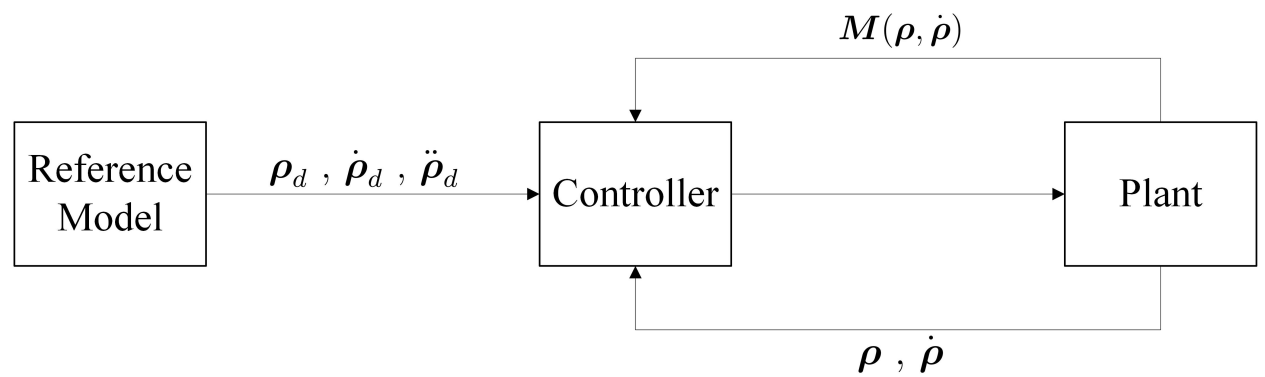

Figure 3.2: NTSMC Block Diagram

The sliding surface for NTSMC is expressed as [19,59]

$$
s=\boldsymbol{e}+\boldsymbol{K} \dot{\boldsymbol{e}}^{\frac{p}{q}}
$$

where the control design matrix, $\boldsymbol{K} \in \mathbb{R}^{3 \times 3}$ is a constant, positive definite matrix.

The positive odd integers $p$ and $q$ must satisfy the following condition $[19,59]$

$$
p>q
$$

In order for the control law to be nonsingular, a second condition must be satisfied, given by the following $[19,59]$

$$
1<\frac{p}{q}<2
$$


Feng et al. [59] explain the reasoning behind the selection of the ratio in Eq. (3.5) and how this ratio causes the system to become nonsingular compared to the traditional TSM.

For the matrix $\dot{\boldsymbol{e}}^{\frac{p}{q}}$, given in Eq. (3.3), the fraction power is given by

$$
\dot{\boldsymbol{e}}^{\frac{p}{q}}=\left[\left(\dot{x}-\dot{x}_{d}\right)^{\frac{p}{q}},\left(\dot{y}-\dot{y}_{d}\right)^{\frac{p}{q}},\left(\dot{z}-\dot{z}_{d}\right)^{\frac{p}{q}}\right]^{T}
$$

The control force for NTSMC, denoted by $\boldsymbol{u}$ is as follows [19]

$$
\boldsymbol{u}=-m_{c}\left(\boldsymbol{M}(\boldsymbol{\rho}, \dot{\boldsymbol{\rho}})+\frac{q}{p} \boldsymbol{K}^{-1} \dot{\boldsymbol{e}}^{2-\frac{p}{q}}-\ddot{\boldsymbol{\rho}}_{d}\right)-\boldsymbol{G}-\boldsymbol{\eta} \operatorname{sgn}(\boldsymbol{s})
$$

where $m_{c}$ is the mass of the chaser, $\boldsymbol{M}(\boldsymbol{\rho}, \dot{\boldsymbol{\rho}})$ is the acceleration of the chaser, $\boldsymbol{\eta} \in \mathbb{R}^{3 \times 3}$ is a diagonal positive definite matrix, and $\operatorname{sgn}(s)$ is

$$
\operatorname{sgn}(\boldsymbol{s})=\left[\operatorname{sgn}\left(s_{x}\right), \operatorname{sgn}\left(s_{y}\right), \operatorname{sgn}\left(s_{z}\right)\right]^{T}
$$

More specifically, $\boldsymbol{M}(\boldsymbol{\rho}, \dot{\boldsymbol{\rho}})$ is defined as

$$
\boldsymbol{M}=\left[\begin{array}{c}
\ddot{x} \\
\ddot{y} \\
\ddot{z}
\end{array}\right]
$$

where these accelerations are given in Eqs. (2.149) to (2.151). The total acceleration affecting the chaser is then

$$
m_{c} \ddot{\boldsymbol{\rho}}=m_{c} \boldsymbol{M}(\boldsymbol{\rho}, \dot{\boldsymbol{\rho}})+\boldsymbol{u}+\boldsymbol{F}_{p}
$$

where $\boldsymbol{F}_{p}=\left[F_{p_{x}}, F_{p_{y}}, F_{p_{z}}\right]^{T}$ is the matrix that defines the external disturbances that act on the chaser. These disturbances were described in Sec. 2.7.

The variable $\boldsymbol{G}$ in Eq. (3.7) is selected such that

$$
\left|F_{p_{i}}\right| \leqslant G_{i}
$$

where $i=x, y, z$. A bound in needed on the perturbation per NTSMC requirements as outlined in [60].

The block diagram for calculating the error, the sliding surface, and the control law is provided in Fig. 3.3. 


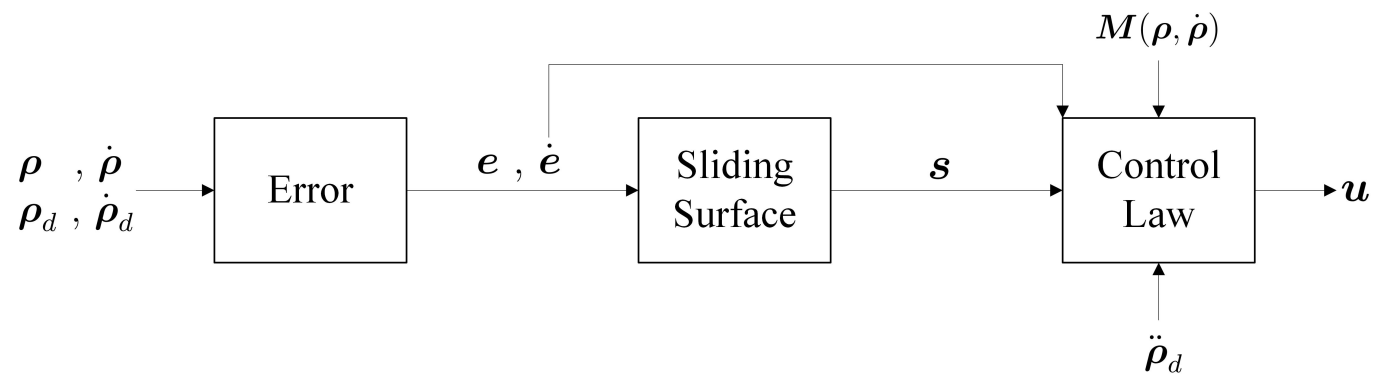

Figure 3.3: Control Law Block Diagram

\subsection{Stability Analysis}

The Lyapunov equation for NTSMC is given by [19]

$$
V=\frac{1}{2} \boldsymbol{s}^{T} m_{c} \boldsymbol{s}
$$

Equation (3.12) is positive semi-definite since $\boldsymbol{s}^{T}$ multiplied by $\boldsymbol{s}$ is always greater than zero, and $m_{c}$ is positive.

Taking the derivative of Eq. (3.12) along its trajectory results in

$$
\dot{V}=s^{T} m_{c} \dot{s}
$$

The derivative of the sliding surface can be calculated using Eq. (3.3). Substituting the derivative in the above results in

$$
\dot{V}=\boldsymbol{s}^{T} m_{c}\left\{\dot{\boldsymbol{e}}+\frac{p}{q} \boldsymbol{K}^{\dot{\boldsymbol{e}}^{q}-1} \ddot{\boldsymbol{e}}\right\}
$$

The variable $\ddot{\boldsymbol{e}}$ is the second derivative of position error, and thus it equals $\ddot{\boldsymbol{\rho}}-\ddot{\boldsymbol{\rho}}_{d}$. Making use of this and performing some algebraic manipulation yields

$$
\dot{V}=\boldsymbol{s}^{T}\left\{m_{c} \dot{\boldsymbol{e}}+\frac{p}{q} \boldsymbol{K} \dot{\boldsymbol{e}}^{\frac{p}{q}-1}\left(m_{c} \ddot{\boldsymbol{\rho}}-m_{c} \ddot{\boldsymbol{\rho}}_{d}\right)\right\}
$$

Substituting Eq. (3.10) for $m_{c} \ddot{\boldsymbol{\rho}}$ gives

$$
\dot{V}=\boldsymbol{s}^{T}\left\{m_{c} \dot{\boldsymbol{e}}+\frac{p}{q} \boldsymbol{K} \dot{\boldsymbol{e}}^{\frac{p}{q}-1}\left(m_{c} \boldsymbol{M}(\boldsymbol{\rho}, \dot{\boldsymbol{\rho}})+\boldsymbol{u}+\boldsymbol{F}_{p}-m_{c} \ddot{\boldsymbol{\rho}}_{d}\right)\right\}
$$

Further manipulation yields

$$
\dot{V}=\boldsymbol{s}^{T}\left[m_{c} \dot{\boldsymbol{e}}+\frac{p}{q} \boldsymbol{K} \dot{\boldsymbol{e}}^{\frac{p}{q}-1}\left\{m_{c} \boldsymbol{M}(\boldsymbol{\rho}, \dot{\boldsymbol{\rho}})+\boldsymbol{u}-m_{c} \ddot{\boldsymbol{\rho}}_{d}\right\}\right]+\frac{p}{q} \boldsymbol{s}^{T} \boldsymbol{C} \dot{\boldsymbol{e}}^{\frac{p}{q}-1} \boldsymbol{F}_{p}
$$


Since the disturbances are assumed to be bounded, one can define $\dot{V}_{1}$ such that $\dot{V}<\dot{V}_{1}$ for $\boldsymbol{F}_{p} \leqslant \boldsymbol{G}[19]$

$$
\dot{V}_{1}=\boldsymbol{s}^{T}\left[m_{c} \dot{\boldsymbol{e}}+\frac{p}{q} \boldsymbol{K} \dot{\boldsymbol{e}}^{\frac{p}{q}-1}\left\{m_{c} \boldsymbol{M}(\boldsymbol{\rho}, \dot{\boldsymbol{\rho}})+\boldsymbol{u}-m_{c} \ddot{\boldsymbol{\rho}}_{d}\right\}\right]+\frac{p}{q} \boldsymbol{s}^{T} \boldsymbol{K} \dot{\boldsymbol{e}}^{\frac{p}{q}-1} \boldsymbol{G}
$$

Making use of the control law, Eq. (3.7), results in

$$
\begin{aligned}
\dot{V}_{1} & =\boldsymbol{s}^{T}\left[m_{c} \dot{\boldsymbol{e}}+\frac{p}{q} \boldsymbol{K} \dot{\boldsymbol{e}}^{\frac{p}{q}-1}\left\{m_{c} \boldsymbol{M}(\boldsymbol{\rho}, \dot{\boldsymbol{\rho}})-m_{c} \ddot{\boldsymbol{\rho}}_{d}\right.\right. \\
& \left.\left.-m_{c}\left(\boldsymbol{M}(\boldsymbol{\rho}, \dot{\boldsymbol{\rho}})+\frac{q}{p} \boldsymbol{K}^{-1} \dot{\boldsymbol{e}}^{2-\frac{p}{q}}-\ddot{\boldsymbol{\rho}}_{d}\right)-\boldsymbol{G}-\boldsymbol{\eta} \operatorname{sgn}(\boldsymbol{s})\right\}\right] \\
& +\frac{p}{q} \boldsymbol{s}^{T} \boldsymbol{K} \dot{\boldsymbol{e}}^{\frac{p}{q}-1} \boldsymbol{G}
\end{aligned}
$$

After some rearrangement the following equation is obtained

$$
\begin{aligned}
\dot{V}_{1} & =\boldsymbol{s}^{T}\left[m_{c} \dot{\boldsymbol{e}}+\frac{p}{q} \boldsymbol{K} \dot{\boldsymbol{e}}^{\frac{p}{q}-1}\left\{m_{c} \boldsymbol{M}(\boldsymbol{\rho}, \dot{\boldsymbol{\rho}})-m_{c} \ddot{\boldsymbol{\rho}}_{d}\right.\right. \\
& \left.\left.-m_{c} \boldsymbol{M}(\boldsymbol{\rho}, \dot{\boldsymbol{\rho}})-m_{c} \frac{q}{p} \boldsymbol{K}^{-1} \dot{\boldsymbol{e}}^{2-\frac{p}{q}}+m_{c} \ddot{\boldsymbol{\rho}}_{d}-\boldsymbol{G}-\boldsymbol{\eta} \operatorname{sgn}(\boldsymbol{s})\right\}\right] \\
& +\frac{p}{q} \boldsymbol{s}^{T} \boldsymbol{K} \dot{\boldsymbol{e}}^{\frac{p}{q}-1} \boldsymbol{G}
\end{aligned}
$$

which can be simplified to

$$
\begin{aligned}
\dot{V}_{1} & =\boldsymbol{s}^{T}\left[m_{c} \dot{\boldsymbol{e}}+\frac{p}{q} \boldsymbol{K} \dot{\boldsymbol{e}}^{\frac{p}{q}-1}\left\{-m_{c} \frac{q}{p} \boldsymbol{K}^{-1} \dot{\boldsymbol{e}}^{2-\frac{p}{q}}-\boldsymbol{G}-\boldsymbol{\eta} \operatorname{sgn}(\boldsymbol{s})\right\}\right] \\
& +\frac{p}{q} \boldsymbol{s}^{T} \boldsymbol{K} \dot{\boldsymbol{e}}^{\frac{p}{q}-1} \boldsymbol{G} \\
& =\boldsymbol{s}^{T}\left[m_{c} \dot{\boldsymbol{e}}-m_{c} \frac{p}{q} \boldsymbol{K} \dot{\boldsymbol{e}}^{\frac{p}{q}-1} \frac{q}{p} \boldsymbol{K}^{-1} \dot{\boldsymbol{e}}^{2-\frac{p}{q}}-\frac{p}{q} \boldsymbol{K} \dot{\boldsymbol{e}}^{\frac{p}{q}-1} \boldsymbol{G}-\frac{p}{q} \boldsymbol{K} \dot{\boldsymbol{e}}^{\frac{p}{q}-1} \boldsymbol{\eta} \operatorname{sgn}(\boldsymbol{s})\right] \\
& +\frac{p}{q} \boldsymbol{s}^{T} \boldsymbol{K} \dot{\boldsymbol{e}}^{\frac{p}{q}-1} \boldsymbol{G}
\end{aligned}
$$

By cancelling like terms, $\dot{V}_{1}$ simplifies to [19]

$$
\dot{V}_{1}=-\boldsymbol{s}^{T} \frac{p}{q} \boldsymbol{K} \dot{\boldsymbol{e}}^{\frac{p}{q}-1} \boldsymbol{\eta} \operatorname{sgn}(\boldsymbol{s})
$$

The variables $p$ and $q$ are both positive, so are the matrices $\boldsymbol{K}$ and $\boldsymbol{\eta}$. The velocity error, $\dot{\boldsymbol{e}}$ is always raised to an even power, making it also positive, and $\boldsymbol{s}$ multiplied by $\operatorname{sgn}(s)$ will also be positive. Therefore $\dot{V}_{1}$ is always less than 0 and so is $\dot{V}$, making the system globally asymptotically stable. 


\subsection{The Chattering Problem}

The chattering phenomenon is defined as an oscillatory motion about the sliding manifold. Chattering is caused due to switching of the sliding surface. Chattering could damage physical system components such as thrusters and actuators [60]. As such, it is important to alleviate this chattering. This can be done by replacing the switching function, $\operatorname{sgn}(s)$, with a saturating function, $\operatorname{sat}(s)$, in the control input equation, Eq. (3.7). This saturating function is given by [60]

$$
\operatorname{sat}\left(s_{i}, \gamma\right)= \begin{cases}s_{i} / \gamma & \left|s_{i}\right|<\gamma \\ \operatorname{sgn}\left(s_{i}\right) & \left|s_{i}\right| \geqslant \gamma\end{cases}
$$

where $\gamma$ is called the boundary layer. Inside this boundary layer, linear feedback control is used and outside of it, the switch control is used [60]. The saturating function is illustrated in Fig. 3.4.

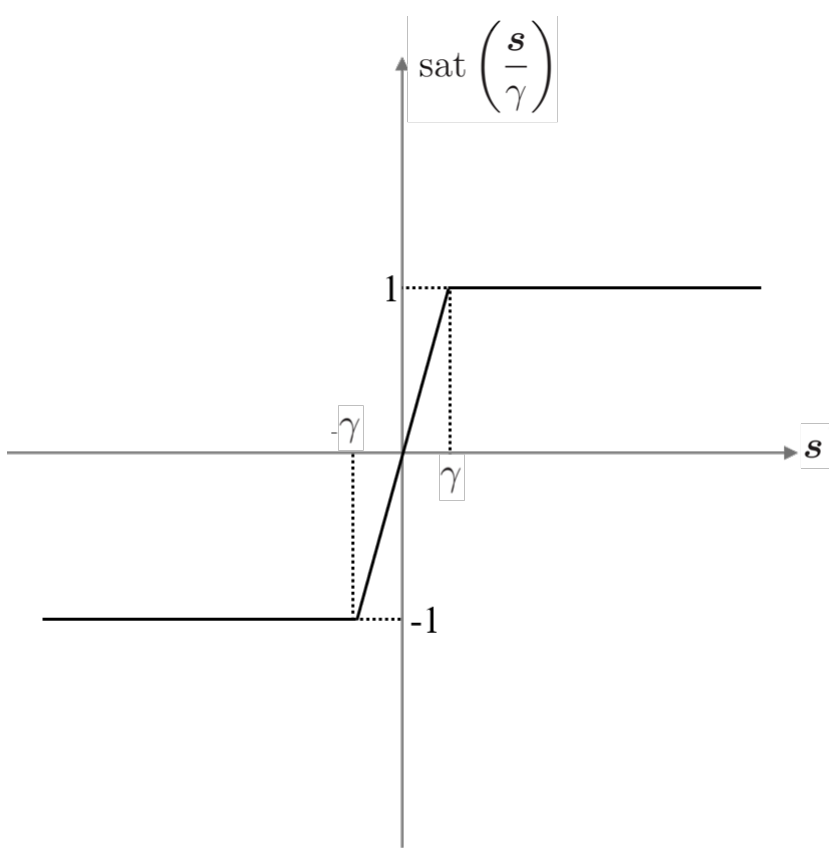

Figure 3.4: Saturation Function, adapted from [60] 


\subsection{Modified Nonsingular Terminal Sliding Mode Control}

Smoothing the effects of the nonlinear switching surface in the control law can improve the precision of the control law [20]. The modified NTSMC (MNTSMC) law, based on the work by Cao et al. [20], is given by

$$
\boldsymbol{u}=-m_{c}\left(\boldsymbol{M}(\boldsymbol{\rho}, \dot{\boldsymbol{\rho}})+\frac{q}{p} \boldsymbol{K}^{-1} \dot{\boldsymbol{e}}^{2-\frac{p}{q}}-\ddot{\boldsymbol{\rho}}_{d}\right)-\frac{1}{2} \boldsymbol{\eta} \boldsymbol{s}
$$

where $\boldsymbol{K}$ and $\boldsymbol{\eta} \in \mathbb{R}^{3 \times 3}$ are diagonal positive definite matrices.

The stability for MNTSMC follows the same procedure as the one explained in Sec. 3.4. The Lyapunov candidate function is as follows [20]

$$
V=\frac{1}{2} \boldsymbol{s}^{T} \boldsymbol{s}
$$

Taking the derivative of $V$ along its trajectory yields

$$
\begin{aligned}
\dot{V} & =\boldsymbol{s}^{T} \dot{\boldsymbol{s}} \\
& =\boldsymbol{s}^{T}\left[\dot{\boldsymbol{e}}+\frac{p}{q} \boldsymbol{K} \dot{\boldsymbol{e}}^{\frac{p}{q}-1} \ddot{\boldsymbol{e}}\right] \\
& =\boldsymbol{s}^{T}\left[\dot{\boldsymbol{e}}+\frac{p}{q} \boldsymbol{K} \dot{\boldsymbol{e}}^{\frac{p}{q}-1}\left\{\boldsymbol{M}(\boldsymbol{\rho}, \dot{\boldsymbol{\rho}})+\frac{1}{m_{c}} \boldsymbol{u}-\ddot{\boldsymbol{\rho}}_{d}\right\}\right] \\
& =\boldsymbol{s}^{T}\left[\dot{\boldsymbol{e}}+\frac{p}{q} \boldsymbol{K} \dot{\boldsymbol{e}}^{\frac{p}{q}-1} \boldsymbol{M}(\boldsymbol{\rho}, \dot{\boldsymbol{\rho}})-\frac{p}{q} \boldsymbol{K} \dot{\boldsymbol{e}}^{\frac{p}{q}-1} \ddot{\boldsymbol{\rho}}_{d}+\frac{p}{q} \frac{1}{m_{c}} \boldsymbol{K} \dot{\boldsymbol{e}}^{\frac{p}{q}-1} \boldsymbol{u}\right] \\
& =\boldsymbol{s}^{T}\left[\dot{\boldsymbol{e}}+\frac{p}{q} \boldsymbol{K} \dot{\boldsymbol{e}}^{\frac{p}{q}-1} \boldsymbol{M}(\boldsymbol{\rho}, \dot{\boldsymbol{\rho}})-\frac{p}{q} \boldsymbol{K} \dot{\boldsymbol{e}}^{\frac{p}{q}-1} \ddot{\boldsymbol{\rho}}_{d}\right. \\
& \left.+\frac{p}{q} \frac{1}{m_{c}} \boldsymbol{K} \dot{\boldsymbol{e}}^{\frac{p}{q}-1}\left\{-m_{c}\left(\boldsymbol{M}(\boldsymbol{\rho}, \dot{\boldsymbol{\rho}})+\frac{q}{p} \boldsymbol{K}^{-1} \dot{\boldsymbol{e}}^{2-\frac{p}{q}}-\ddot{\boldsymbol{\rho}}_{d}\right)-\frac{1}{2} \boldsymbol{\eta} \boldsymbol{s}\right\}\right] \\
& =-\boldsymbol{s}^{T} \frac{p}{2 q} \frac{1}{m_{c}} \boldsymbol{K} \dot{\boldsymbol{e}}^{\frac{p}{q}-1} \boldsymbol{\eta} \boldsymbol{s}
\end{aligned}
$$

$\dot{V}$ is negative definite following the same argument as in Sec. 3.4. 


\subsection{Finite Time Analysis}

Initially, if the sliding surface is not zero, the system will reach zero in finite time. That is the time it takes to get from $s(0) \neq 0$ to $s=0$, is given by [60]

$$
t_{r} \leqslant \frac{|s(0)|}{\eta}
$$

where $t_{r}$ is the reaching time.

When the system reaches the sliding surface, that is when $s=0$, the system dynamics can be expressed by

$$
\boldsymbol{s}=\boldsymbol{e}+\boldsymbol{K} \dot{\boldsymbol{e}}^{\frac{p}{q}}=0
$$

Once the system reaches the sliding surface, the error is not necessarily zero; that is $\boldsymbol{e} \neq 0$. The time for the system to get from the reaching phase to the sliding phase, $\boldsymbol{e}\left(t_{r}\right)$ to $\boldsymbol{e}\left(t_{r}+t_{s}\right)$, can be found by first rearranging the above such that

$$
e=-\boldsymbol{K} \dot{e}^{\frac{p}{q}}
$$

which can then be manipulated into

$$
e_{i}^{\frac{q}{p}}=-\boldsymbol{K}^{\frac{q}{p}} \frac{d e_{i}}{d t}
$$

where $i=x, y, z$.

After further manipulation, and integrating $d t$ gives

$$
t_{s i}=-\boldsymbol{K}^{\frac{q}{p}} \int_{e_{i}\left(t_{r i}\right)}^{0} \frac{d e_{i}}{e_{i}^{\frac{q}{p}}}
$$

Thus the sliding time, denoted by $t_{s i}$, is then given by

$$
t_{s i}=-\boldsymbol{K}^{\frac{q}{p}} \frac{p}{p-q}\left[\left|e_{i}(0)^{\frac{p-q}{p}}\right|-\left|e_{i}\left(t_{r i}\right)^{\frac{p-q}{p}}\right|\right]
$$




\section{Chapter 4}

\section{Optimal Sliding Mode Control}

This chapter presents optimal sliding mode control (OSMC) law. OSCM is the third control law that will be used as a benchmark in this thesis. OSMC is a combination of optimal control law, linear quadratic regulator (LQR), and integral sliding mode control (ISMC). For this control law, first the optimal control law is presented. Then ISMC is used to improve the performance of the control law. ISMC belongs to the family of SMC, previously explained in Sec. 3.1. The biggest advantage of ISMC is the elimination of the reaching phase. The system is therefore on the sliding surface from the very beginning, making ISMC more robust to disturbances compared to other SMC laws that have the reaching phase. Different types of ISMC were used in $[27,46]$ for fault tolerant systems; however, OSMC has never been tested for faults.

To begin the implementation, first the simplified equations of motion, previously explained in Sec. 2.8, are implemented in the plant. This is detailed in Sec. 4.1: SFF Dynamics Modification. Then, the tracking error for OSMC is presented in Sec. 4.2: Tracking Error. This is followed by the definition of optimal control theory and the derivation of OSMC in Secs. 4.3: Optimal Control Law and 4.4: Integral Sliding Mode Control, respectively. This chapter is concluded by the stability analysis of OSMC based on Lyapunov's second method in Sec. 4.5: Stability Analysis. 


\subsection{SFF Dynamics Modification}

Using the simplified dynamics of SFF, Eqs. (2.149) to (2.151), the state space representation is expressed as

$$
\begin{gathered}
\dot{\boldsymbol{x}}(t)=\tilde{\boldsymbol{A}}(t) \boldsymbol{x}(t)+\boldsymbol{B} \boldsymbol{u}(t)+\boldsymbol{a}_{p} \\
\boldsymbol{y}(t)=\boldsymbol{x}(t)
\end{gathered}
$$

where $\tilde{\boldsymbol{A}}(t)$ is as follows

$$
\tilde{\boldsymbol{A}}(t)=\left[\begin{array}{cccccc}
0 & 0 & 0 & 1 & 0 & 0 \\
0 & 0 & 0 & 0 & 1 & 0 \\
0 & 0 & 0 & 0 & 0 & 1 \\
\dot{\theta}^{2}+\frac{2 \mu}{r_{t}^{3}} & \ddot{\theta} & 0 & 0 & 2 \dot{\theta} & 0 \\
-\ddot{\theta} & \dot{\theta}^{2}-\frac{\mu}{r_{t}^{3}} & 0 & -2 \dot{\theta} & 0 & 0 \\
0 & 0 & -\frac{\mu}{r_{t}^{3}} & 0 & 0 & 0
\end{array}\right]
$$

For OSMC, the desired trajectory is given by

$$
\dot{\boldsymbol{x}}(t)_{d}=\tilde{\boldsymbol{A}}(t) \boldsymbol{x}_{d}(t)
$$

and

$$
\boldsymbol{y}_{d}(t)=\boldsymbol{x}_{d}(t)
$$

where $\boldsymbol{x}_{d}$ and $\boldsymbol{y}_{d} \in \mathbb{R}^{6 \times 1}$.

\subsection{Tracking Error}

For OSMC, the tracking error is described as such [44]

$$
\boldsymbol{e}(t)=\boldsymbol{y}(t)-\boldsymbol{y}_{d}(t)
$$

Taking the derivative of Eq. (4.6) yields

$$
\dot{\boldsymbol{e}}(t)=\dot{\boldsymbol{y}}(t)-\dot{\boldsymbol{y}}_{d}(t)
$$

Making use of Eqs. (4.2) and (4.5) results in [44]

$$
\dot{\boldsymbol{e}}(t)=\tilde{\boldsymbol{A}}(t) \boldsymbol{x}(t)+\boldsymbol{B} \boldsymbol{u}(t)+\boldsymbol{a}_{p}-\tilde{\boldsymbol{A}}(t) \boldsymbol{x}_{d}(t)
$$

which can then be simplified to

$$
\dot{\boldsymbol{e}}(t)=\tilde{\boldsymbol{A}}(t) \boldsymbol{e}(t)+\boldsymbol{B} \boldsymbol{u}(t)+\boldsymbol{a}_{p}
$$




\subsection{Optimal Control Law}

This section highlights optimal control theory. Linear quadratic regulator (LQR) is an optimal multivariable feedback control law that allows one to minimize the error of a system while requiring a minimum controller effort. This minimization is done through appropriate selection of two gain matrices [61]. The linear state feedback control law is given by [62]

$$
\boldsymbol{u}(t)=-\boldsymbol{K} \boldsymbol{e}(t)
$$

The state feedback control law minimizes the following quadratic performance index cost function [62]

$$
\boldsymbol{J}(\boldsymbol{e}(t), \boldsymbol{u}(t))=\int_{0}^{\infty}\left(\boldsymbol{e}(t)^{T} \boldsymbol{Q}(t) \boldsymbol{e}(t)+\boldsymbol{u}(t)^{T} \boldsymbol{R}(t) \boldsymbol{u}(t)\right) d t
$$

where $\boldsymbol{Q} \in \mathbb{R}^{6 \times 6}$ is a positive semi-definite state weighing matrix, where large values contribute to prioritizing the control error, and where $\boldsymbol{R} \in \mathbb{R}^{3 \times 3}$ is a positive definite control weighing matrix, whose elements are selected to be large if control effort is prioritized [44].

The control gain, $\boldsymbol{K}$, in Eq. (4.10) is expressed as

$$
\boldsymbol{K}=\boldsymbol{R}^{-1} \boldsymbol{B}^{T} \boldsymbol{P}(t)
$$

where $\boldsymbol{P} \in \mathbb{R}^{6 \times 6}$ is a positive semi-definite matrix that is the solution to the algebraic Riccati equation given by [62]

$$
\boldsymbol{P}(t) \tilde{\boldsymbol{A}}(t)+\tilde{\boldsymbol{A}}(t)^{T} \boldsymbol{P}(t)-\boldsymbol{P}(t) \boldsymbol{B} \boldsymbol{R}^{-1} \boldsymbol{B}^{T} \boldsymbol{P}(t)+\boldsymbol{Q}=0
$$

Therefore, the control law can be rewritten as

$$
\boldsymbol{u}(t)=-\boldsymbol{R}^{-1} \boldsymbol{B}^{T} \boldsymbol{P}(t) \boldsymbol{e}(t)
$$

Conventional optimal control, as given is Eq. (4.14) is designed such that it only stabilizes the system without considering disturbances [44]. Substituting in the control law from Eq. (4.14) into Eq. (4.9) and ignoring the disturbances gives [44]

$$
\dot{\boldsymbol{e}}(t)=\left(\tilde{\boldsymbol{A}}(t)-\boldsymbol{B} \boldsymbol{R}^{-1} \boldsymbol{B}^{T} \boldsymbol{P}(t)\right) \boldsymbol{e}(t)
$$




\subsection{Integral Sliding Mode Control}

Figure 4.1 shows the overall block diagram for OSMC.

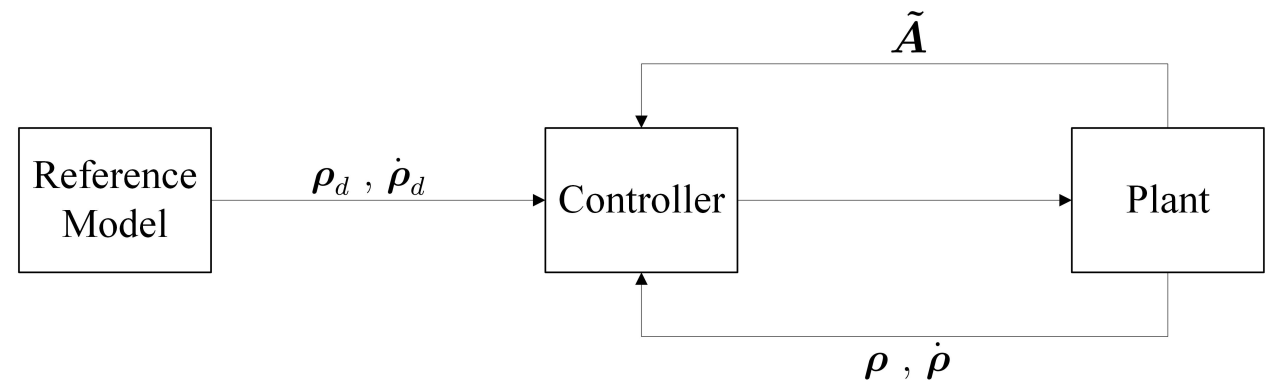

Figure 4.1: OSMC Block Diagram

For ISMC, the nonlinear integral sliding surface is defined as $[44,63]$

$$
\boldsymbol{s}(\boldsymbol{e}(t), t)=\boldsymbol{M}(\boldsymbol{e}(t)-\boldsymbol{e}(0))-\boldsymbol{M} \int_{0}^{t}\left(\tilde{\boldsymbol{A}}(t)-\boldsymbol{B} \boldsymbol{R}^{-1} \boldsymbol{B}^{T} \boldsymbol{P}(t)\right) \boldsymbol{e}(t) d t
$$

where $\boldsymbol{M} \in \mathbb{R}^{3 \times 6}$ is selected such that $\boldsymbol{M} \boldsymbol{B}$ is nonsingular [44] and $\boldsymbol{e}(0)$ is the initial error. The presence of the initial error in the sliding surface equation is the underlying reason behind the elimination of the reaching phase.

Taking the derivative of Eq. (4.16) gives

$$
\dot{\boldsymbol{s}}(\boldsymbol{e}(t), t)=\boldsymbol{M} \dot{\boldsymbol{e}}(t)-\boldsymbol{M}\left(\tilde{\boldsymbol{A}}(t)-\boldsymbol{B} \boldsymbol{R}^{-1} \boldsymbol{B}^{T} \boldsymbol{P}\right) \boldsymbol{e}(t)
$$

Making use of Eq. (4.9) and ignoring the disturbances yields

$$
\dot{\boldsymbol{s}}(\boldsymbol{e}(t), t)=\boldsymbol{M}(\tilde{\boldsymbol{A}}(t) \boldsymbol{e}+\boldsymbol{B} \boldsymbol{u}(t))-\boldsymbol{M}\left(\tilde{\boldsymbol{A}}(t)-\boldsymbol{B} \boldsymbol{R}^{-1} \boldsymbol{B}^{T} \boldsymbol{P}\right) \boldsymbol{e}(t)
$$

Given the definition of SMC, both $\boldsymbol{s}$ and $\dot{\boldsymbol{s}}$ must equal zero as the system reaches the sliding surface. Thus for Eq. (4.18) to equate to zero, the control law should be [44]

$$
\boldsymbol{u}(t)=-\boldsymbol{R}^{-1} \boldsymbol{B}^{T} \boldsymbol{P}(t) \boldsymbol{e}(t)
$$

which is the same as the one for optimal control, given in Eq. (4.14).

To further improve the performance of the control law, Eq. (4.19) is modified as such [44]

$$
\boldsymbol{u}(t)=-\boldsymbol{R}^{-1} \boldsymbol{B}^{T} \boldsymbol{P}(t) \boldsymbol{e}(t)-(\boldsymbol{M} \boldsymbol{B})^{-1} \boldsymbol{\lambda} \operatorname{sgn}(\boldsymbol{s})
$$


where $\boldsymbol{\lambda} \in \mathbb{R}^{3 \times 3}$ is a diagonal gain matrix, and $\operatorname{sgn}(\boldsymbol{s})$ is

$$
\operatorname{sgn}(\boldsymbol{s})=\left[\operatorname{sgn}\left(s_{x}\right), \operatorname{sgn}\left(s_{y}\right), \operatorname{sgn}\left(s_{z}\right)\right]^{T}
$$

The block diagram for calculating the error, the sliding surface, and the control law is provided in Fig. 4.2.

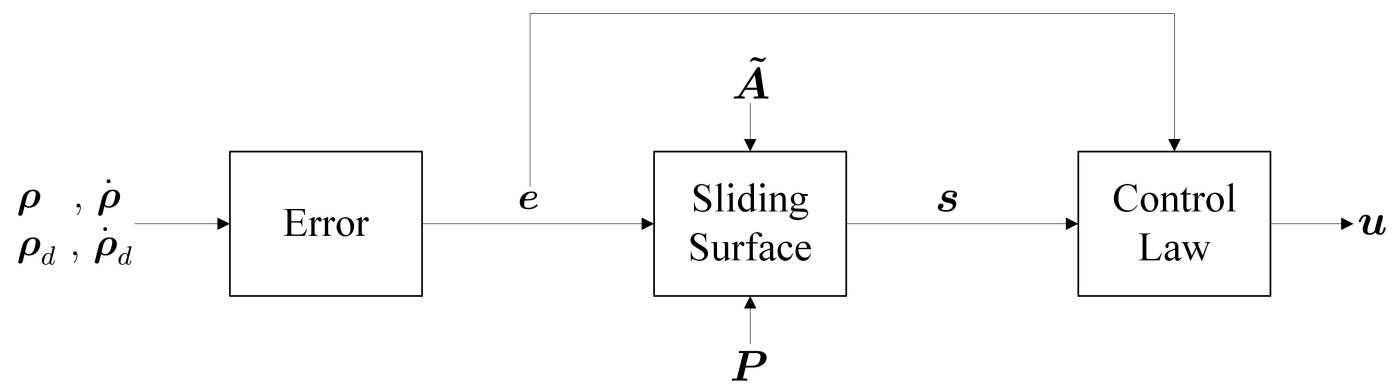

Figure 4.2: Control Law Block Diagram 


\subsection{Stability Analysis}

The Lyapunov function for OSMC is given by [44]

$$
V=\frac{1}{2} \boldsymbol{s}^{T} \boldsymbol{s}
$$

Equation (4.22) is positive semi-definite at all times as $\boldsymbol{s}^{T} \boldsymbol{s}$ will always be greater than or equal to zero.

Taking the derivative of Eq. (4.22) along its trajectory gives

$$
\dot{V}=s^{T} \dot{s}
$$

Substituting in Eq. (4.17) for $\dot{\boldsymbol{s}}$ yields

$$
\dot{V}=\boldsymbol{s}^{T}\left\{\boldsymbol{M} \dot{\boldsymbol{e}}(t)-\boldsymbol{M}\left[\tilde{\boldsymbol{A}}(t)-\boldsymbol{B} \boldsymbol{R}^{-1} \boldsymbol{B}^{T} \boldsymbol{P}(t)\right] \boldsymbol{e}(t)\right\}
$$

where $\dot{\boldsymbol{e}}$ is given by Eq. (4.9).

Replacing $\boldsymbol{u}$ in Eq. (4.9) with the control law from Eq. (4.20) results in

$$
\begin{aligned}
\dot{V} & =\boldsymbol{s}^{T}\{\boldsymbol{M}[\tilde{\boldsymbol{A}}(t) \boldsymbol{e}(t) \\
& \left.+\boldsymbol{B}\left(-\boldsymbol{R}^{-1} \boldsymbol{B}^{T} \boldsymbol{P}(t) \boldsymbol{e}(t)-(\boldsymbol{M} \boldsymbol{B})^{-1} \boldsymbol{\eta} \operatorname{sgn}(\boldsymbol{s})\right)+\boldsymbol{a}_{p}\right] \\
& \left.-\boldsymbol{M}\left[\tilde{\boldsymbol{A}}(t)-\boldsymbol{B} \boldsymbol{R}^{-1} \boldsymbol{B}^{T} \boldsymbol{P}(t)\right] \boldsymbol{e}(t)\right\}
\end{aligned}
$$

After cancelling like terms, the above simplifies to [44]

$$
\dot{V}=\boldsymbol{s}^{T}\left\{\boldsymbol{M} \boldsymbol{a}_{p}-\boldsymbol{M B}(\boldsymbol{M B})^{-1} \boldsymbol{\lambda} \operatorname{sgn}(\boldsymbol{s})\right\}
$$

By letting $\boldsymbol{M}=\boldsymbol{B}^{T}$, Eq. (4.26) can be further simplified to [44]

$$
\dot{V}=\boldsymbol{s}^{T}\left\{\boldsymbol{M} \boldsymbol{a}_{p}-\boldsymbol{\lambda} \operatorname{sgn}(\boldsymbol{s})\right\}
$$

Assuming that the perturbations are bounded such that $a_{P i} \leqslant d_{i}$, where $i=x, y, z$, one can define $\dot{V}_{1}$ such that $\dot{V}<\dot{V}_{1}$. Thus

$$
\dot{V}_{1}=s^{T}\{\boldsymbol{M d}-\boldsymbol{\lambda} \operatorname{sgn}(\boldsymbol{s})\}
$$


Equation (4.28) can be rewritten in terms of its components as

$$
V_{1_{i}}=\frac{s_{i} d_{i}}{m_{c}}-\left|s_{i}\right| \lambda_{i}
$$

By choosing $s_{i} d_{i}<m_{c} \eta_{i}$, the system will be globally asymptotically stable, where $i=x, y, z$. 


\section{Chapter 5}

\section{Simple Adaptive Control}

This chapter presents the final control law in this thesis, called simple adaptive control (SAC). The adaptive nature of this control law allows the controller to modify its structure and parameters in real-time to efficiently manage unexpected actuator faults, dynamics uncertainties, and orbital perturbations. Unlike SMC, this control law does not require any information regarding the dynamics of the system. Being model-free is a great benefit of SAC.

$\mathrm{SAC}$ is based on model reference adaptive control (MRAC). It was first introduced by Sobel et al. [64] and further developed by Barkana [65, 66]. Compared to MRAC, SAC does not require the desired model to be of the same order as the plant. Additionally, unlike MRAC which could be unstable even when the plant and the model are stable, SAC will guarantee stability due to the passivity conditions $[67,68]$.

The rest of this chapter is as follows. The ideal model is explained in Sec. 5.1: Ideal Model. Section 5.2: Control Law presents the control law for SAC. The passivity condition of the relative dynamics in the plant is detailed in Sec. 5.3: Almost Strictly Passive Plant. The definition of the ideal plant and its comparison to the actual plant is provided in Sec. 5.4: Ideal Plant and the stability proof of SAC is presented in Sec. 5.5: Stability Analysis using Lyapunov stability theory and LaSalle's invariance principle. 


\subsection{Ideal Model}

The plant is required to follow a model, called the ideal model. The overview of the ideal model is shown in Fig. 5.1.

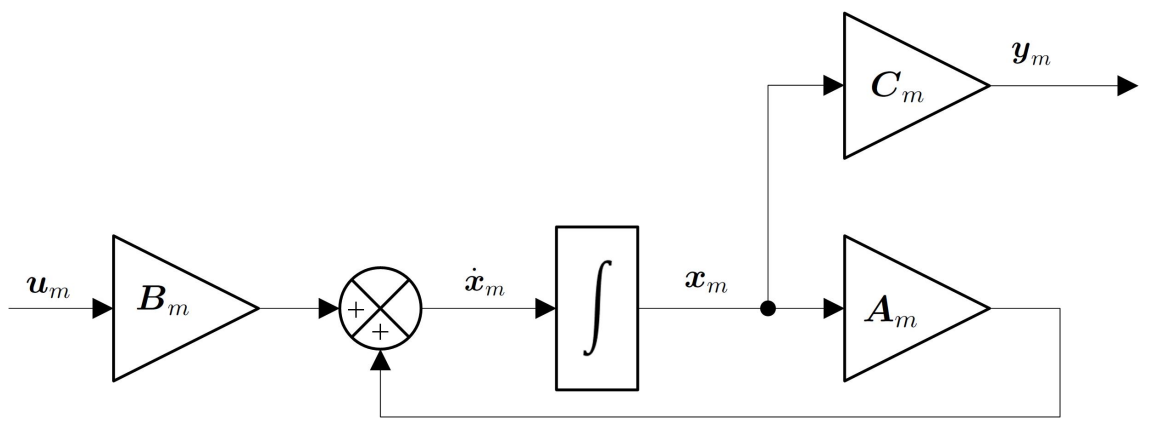

Figure 5.1: Ideal Model Representation

The ideal model is mathematically expressed as [65]

$$
\begin{gathered}
\dot{\boldsymbol{x}}_{m}=\boldsymbol{A}_{m} \boldsymbol{x}_{m}+\boldsymbol{B}_{m} \boldsymbol{u}_{m} \\
\boldsymbol{y}_{m}=\boldsymbol{C}_{m} \boldsymbol{x}_{m}
\end{gathered}
$$

where the state denoted by $\boldsymbol{x}_{m} \in \mathbb{R}^{6 \times 1}$, the inputs denoted by $\boldsymbol{u}_{m} \in \mathbb{R}^{3 \times 1}$, and the outputs denoted by $\boldsymbol{y}_{m} \in \mathbb{R}^{3 \times 1}$ are defined as [69]

$$
\begin{aligned}
& \boldsymbol{x}_{m}=\left[\begin{array}{llllll}
x_{m} & y_{m} & z_{m} & \dot{x}_{m} & \dot{y}_{m} & \dot{z}_{m}
\end{array}\right]^{T} \\
& \boldsymbol{u}_{m}=\left[\begin{array}{lll}
x_{d} & y_{d} & z_{d}
\end{array}\right]^{T} \\
& \boldsymbol{y}_{m}=\left[\begin{array}{lll}
\alpha x_{m}+\dot{x}_{m} & \alpha y_{m}+\dot{y}_{m} & \alpha z_{m}+\dot{z}_{m}
\end{array}\right]^{T}
\end{aligned}
$$

where $\boldsymbol{u}_{m}=\boldsymbol{\rho}_{d}$, which corresponds to the desired components of the position and $\alpha$ is a scaling factor. The scaling factor is required so that the dimension of the output of the ideal model matches the dimension of the output of the plant, which is to be discussed in the next section. 
The ideal model matrices, $\boldsymbol{A}_{m}, \boldsymbol{B}_{m}$, and $\boldsymbol{C}_{m}$ are defined as [69]

$$
\begin{gathered}
\boldsymbol{A}_{m}=\left[\begin{array}{cccccc}
0 & 0 & 0 & 1 & 0 & 0 \\
0 & 0 & 0 & 0 & 1 & 0 \\
0 & 0 & 0 & 0 & 0 & 1 \\
-\omega_{n}^{2} & 0 & 0 & -2 \zeta \omega_{n} & 0 & 0 \\
0 & -\omega_{n}^{2} & 0 & 0 & -2 \zeta \omega_{n} & 0 \\
0 & 0 & -\omega_{n}^{2} & 0 & 0 & -2 \zeta \omega_{n}
\end{array}\right] \\
\boldsymbol{B}_{m}=\left[\begin{array}{llllcc}
0 & 0 & 0 & \omega_{n}^{2} & 0 & 0 \\
0 & 0 & 0 & 0 & \omega_{n}^{2} & 0 \\
0 & 0 & 0 & 0 & 0 & \omega_{n}^{2}
\end{array}\right]^{T} \\
\boldsymbol{C}_{m}=\left[\begin{array}{lll}
\alpha \boldsymbol{I}_{3} & \boldsymbol{I}_{3}
\end{array}\right]
\end{gathered}
$$

where $\omega_{n}$ is the natural frequency, $\zeta$ is the damping coefficient, and $\alpha$ is the same scaling factor as in Eq. (5.5). 


\subsection{Control Law}

This section presents the control law for SAC. The adaptive control gains are given by [65]

$$
\begin{aligned}
& \boldsymbol{K}_{e}(t)=\boldsymbol{K}_{I_{e}}(t)+\boldsymbol{K}_{P_{e}}(t) \\
& \boldsymbol{K}_{u}(t)=\boldsymbol{K}_{I_{u}}(t)+\boldsymbol{K}_{P_{u}}(t) \\
& \boldsymbol{K}_{x}(t)=\boldsymbol{K}_{I_{x}}(t)+\boldsymbol{K}_{P_{x}}(t)
\end{aligned}
$$

where $\boldsymbol{K}_{e}(t) \in \mathbb{R}^{3 \times 3}$ is the adaptive control gain that maintains the stability of the closed loop and $\boldsymbol{K}_{u}(t) \in \mathbb{R}^{3 \times 3}$ and $\boldsymbol{K}_{x}(t) \in \mathbb{R}^{3 \times 6}$ are the feedforward adaptive gains which contribute to driving the tracking error, $\boldsymbol{e}_{y}$, to zero [69].

Specifically, the tracking error, $\boldsymbol{e}_{y}$ is given by

$$
\boldsymbol{e}_{y} \triangleq \boldsymbol{y}_{m}-\boldsymbol{y}
$$

where $\boldsymbol{y}$ is expressed as

$$
\boldsymbol{y}=\left[\begin{array}{lll}
\alpha x+\dot{x} & \alpha y+\dot{y} & \alpha z+\dot{z}
\end{array}\right]^{T}
$$

where $x$ to $\dot{z}$ come from the plant and $\alpha$ is the same scaling factor as in Eq. (5.5). The output matrix is modelled as a linear combination to end up with a square state space representation. A square state space representation is required for the passivity condition; the passivity condition can only be achieved by having a square state space representation.

The integral terms of the control gains are obtained as such [65]

$$
\begin{aligned}
\dot{\boldsymbol{K}}_{I_{e}}(t) & =\boldsymbol{e}_{y}(t) \boldsymbol{e}_{y}^{T}(t) \Gamma_{I e} \\
\dot{\boldsymbol{K}}_{I_{u}}(t) & =\boldsymbol{e}_{y}(t) \boldsymbol{u}_{m}^{T}(t) \Gamma_{I u} \\
\dot{\boldsymbol{K}}_{I_{x}}(t) & =\boldsymbol{e}_{y}(t) \boldsymbol{x}_{m}^{T}(t) \Gamma_{I x}
\end{aligned}
$$

and proportional terms of the control gains are obtained as such

$$
\begin{aligned}
& \boldsymbol{K}_{P_{e}}(t)=\boldsymbol{e}_{y}(t) \boldsymbol{e}_{y}^{T}(t) \Gamma_{P e} \\
& \boldsymbol{K}_{P_{u}}(t)=\boldsymbol{e}_{y}(t) \boldsymbol{u}_{m}^{T}(t) \Gamma_{P u} \\
& \boldsymbol{K}_{P_{x}}(t)=\boldsymbol{e}_{y}(t) \boldsymbol{x}_{m}^{T}(t) \Gamma_{P x}
\end{aligned}
$$


where $\Gamma_{I e}, \Gamma_{I u} \in \mathbb{R}^{3 \times 3}, \Gamma_{I x} \in \mathbb{R}^{6 \times 6}$ are the positive definite diagonal matrices that set the adaptation rate for the integral adaptive control gains and $\Gamma_{P e}, \Gamma_{P u} \in \mathbb{R}^{3 \times 3}$, and $\Gamma_{P u} \in \mathbb{R}^{6 \times 6}$ are the positive definite diagonal matrices that set the adaptation rate for the proportional adaptive control gains.

The total control input is then given by

$$
\boldsymbol{u}=\boldsymbol{K}_{e}(t) \boldsymbol{e}_{y}+\boldsymbol{K}_{x}(t) \boldsymbol{x}_{m}+\boldsymbol{K}_{u}(t) \boldsymbol{u}_{m}
$$

Equation (5.20) can be rewritten in a more concise format as such

$$
\boldsymbol{u}=\boldsymbol{K}(t) \boldsymbol{r}
$$

where $\boldsymbol{K}$ and $\boldsymbol{r}$ are

$$
\begin{gathered}
\boldsymbol{K}(t)=\boldsymbol{K}_{I}(t)+\boldsymbol{K}_{P}(t) \\
\boldsymbol{r}=\left[\begin{array}{lll}
\boldsymbol{e}_{y}^{T} & \boldsymbol{x}_{m}^{T} & \boldsymbol{u}_{m}^{T}
\end{array}\right]^{T}
\end{gathered}
$$

Figure 5.2 shows the overview of SAC.

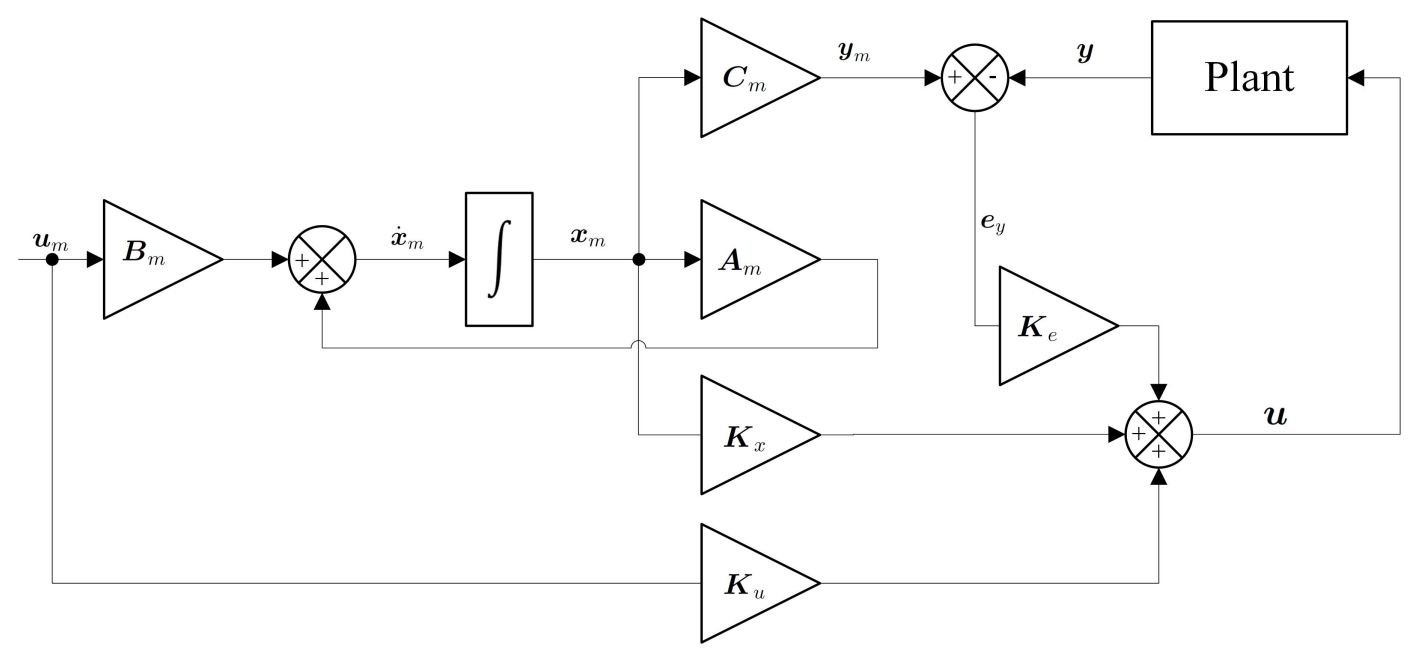

Figure 5.2: Simple Adaptive Control Diagram

\section{Sigma Term}

The tracking error eventually decreases but will not become zero. The integral value for $\boldsymbol{K}_{I_{e}}(t)$ will therefore never become zero and an integration error will be built up over time [70,71]. The integration error built up can be prevented by using the sigma term, also referred to as forgetting factor, which is used to lower the integral 
value. It has been observed that $\boldsymbol{K}_{I_{u}}(t)$ and $\boldsymbol{K}_{I_{x}}(t)$ perform better to minimize the tracking error without the $\sigma$ term. Thus, $\sigma$ is only added for $\boldsymbol{K}_{I_{e}}(t)$ as such [70]

$$
\dot{\boldsymbol{K}}_{I_{e}}(t)=\boldsymbol{e}_{y}(t) \boldsymbol{e}_{y}^{T}(t) \Gamma_{I e}-\sigma \boldsymbol{K}_{I_{e}}(t)
$$

where $\sigma$ is chosen to be a very small number.

\subsection{Almost Strictly Passive Plant}

This section presents the proof of passivity conditions, which is eventually needed for the stability analysis. Consider a modified plant with representation

$$
\begin{gathered}
\dot{\boldsymbol{x}}=\boldsymbol{A} \boldsymbol{x}+\boldsymbol{B u} \\
\boldsymbol{y}=\boldsymbol{C} \boldsymbol{x}
\end{gathered}
$$

where $\boldsymbol{u}$ is the control input and $\boldsymbol{y}$ is given in Eq. (5.13).

Definition 1: The system is considered to be almost strictly passive (ASP) if the product of $\boldsymbol{C} \boldsymbol{B}$ is positive definite symmetric (PDS). The ASP system satisfies the following two conditions [68].

$$
\begin{gathered}
\dot{\boldsymbol{P}}+\boldsymbol{P}\left[\boldsymbol{A}-\boldsymbol{B} \widetilde{\boldsymbol{K}}_{e} \boldsymbol{C}\right]+\left[\boldsymbol{A}-\boldsymbol{B} \widetilde{\boldsymbol{K}}_{e} \boldsymbol{C}\right]^{T} \boldsymbol{P}=-\boldsymbol{Q} \\
\boldsymbol{P} \boldsymbol{B}=\boldsymbol{C}^{T}
\end{gathered}
$$

where $\boldsymbol{P}$ and $\boldsymbol{Q}$ are two PDS matrices and $\widetilde{\boldsymbol{K}}_{\boldsymbol{e}}$ is the constant output feedback gain matrix [71].

Property 1: The state space representation of the nonlinear spacecraft formation flying dynamics model in Eq. (2.155) is ASP.

Proof: The product of $\boldsymbol{C B}$ is PDS as seen in the following.

$$
\boldsymbol{C B}=\left[\begin{array}{ll}
\alpha \boldsymbol{I}_{3} & \boldsymbol{I}_{3}
\end{array}\right]\left[\begin{array}{ccc}
0 & 0 & 0 \\
0 & 0 & 0 \\
0 & 0 & 0 \\
\frac{1}{m_{c}} & 0 & 0 \\
0 & \frac{1}{m_{c}} & 0 \\
0 & 0 & \frac{1}{m_{c}}
\end{array}\right]=\frac{1}{m_{c}} \boldsymbol{I}_{3}
$$


Property 2: The state space representation in Eqs. (5.25)-(5.26) is said to be uniformly strictly minimum-phase if its zero dynamics is uniformly asymptotically stable [68].

Proof: There exist two matrices $\boldsymbol{M} \in \mathbb{R}^{6 \times 3}$ and $\boldsymbol{N} \in \mathbb{R}^{3 \times 6}$, called non-stationary matrices, that satisfy [68]

$$
\begin{gathered}
C M=0_{3} \\
N B=0_{3} \\
N M=I_{3}
\end{gathered}
$$

First the following transformation is defined [68].

$$
\boldsymbol{x}=\boldsymbol{M z}
$$

Using Eq. (5.32), the matrix $\boldsymbol{z}$ can be expressed as

$$
z=N \boldsymbol{M}=\boldsymbol{N} \boldsymbol{x}
$$

Taking the derivative of Eq. (5.34) yields

$$
\dot{z}=\dot{N} \boldsymbol{x}+N \dot{x}
$$

Making use of Eqs. (5.25), (5.31), and (5.33) gives

$$
\begin{aligned}
\dot{\boldsymbol{z}} & =\dot{\boldsymbol{N}} \boldsymbol{x}+\boldsymbol{N}(\boldsymbol{A} \boldsymbol{x}+\boldsymbol{B u}) \\
& =\dot{\boldsymbol{N}} \boldsymbol{x}+\boldsymbol{N} \boldsymbol{A} \boldsymbol{x}+\boldsymbol{N} \boldsymbol{B} \boldsymbol{u} \\
& =\dot{\boldsymbol{N}} \boldsymbol{x}+\boldsymbol{N} \boldsymbol{A} \boldsymbol{x} \\
& =\boldsymbol{N} \boldsymbol{A} \boldsymbol{x} \\
& =\boldsymbol{N} \boldsymbol{A} \boldsymbol{M} \boldsymbol{z}
\end{aligned}
$$

The zero dynamics is then given by

$$
\dot{\boldsymbol{z}}=\boldsymbol{A}_{z} \boldsymbol{z} \in \mathbb{R}^{3 \times 3}
$$

where $\boldsymbol{A}_{z}$, the zero dynamics matrix is

$$
\boldsymbol{A}_{z}=\boldsymbol{N} \boldsymbol{A} \boldsymbol{M}
$$

as previously defined in Eq. (5.36). 
The matrices $\boldsymbol{M}$ and $\boldsymbol{N}$ that satisfy Eqs. (5.30)-(5.32) are

$$
\begin{aligned}
& \boldsymbol{M}=\left[\begin{array}{c}
\boldsymbol{I}_{3} \\
-\alpha \boldsymbol{I}_{3}
\end{array}\right] \\
& \boldsymbol{N}=\left[\begin{array}{ll}
\boldsymbol{I}_{3} & \mathbf{0}_{3}
\end{array}\right]
\end{aligned}
$$

Substituting the above into Eq. (5.38) yields

$$
\boldsymbol{A}_{z}=\boldsymbol{N} \boldsymbol{A} \boldsymbol{M}=-\alpha \boldsymbol{I}_{3}
$$

Equation (5.37) can then be rewritten as

$$
\dot{z}=-\alpha \boldsymbol{z}
$$

Equation (5.42) shows that the zero dynamics is stable and that the relative motion dynamics is uniformly strictly minimum phase $[68,69]$. 


\subsection{Ideal Plant}

When the plant perfectly tracks the ideal model, the plant is called ideal. The ideal plant is represented by [68]

$$
\begin{gathered}
\dot{x}^{*}=A^{*} \boldsymbol{x}^{*}+\boldsymbol{B}^{*} \boldsymbol{u}^{*} \\
\boldsymbol{y}^{*}=\boldsymbol{C} \boldsymbol{x}^{*}
\end{gathered}
$$

where $\boldsymbol{u}^{*}$ is

$$
\boldsymbol{u}^{*}=\widetilde{\boldsymbol{K}}_{x} \boldsymbol{x}_{m}+\widetilde{\boldsymbol{K}}_{u} \boldsymbol{u}_{m}
$$

and where $\widetilde{\boldsymbol{K}}_{x}$ and $\widetilde{\boldsymbol{K}}_{u}$ are constant ideal feedforward gains.

The state error, $\boldsymbol{e}_{x}$, is defined as [68]

$$
\boldsymbol{e}_{x}=\boldsymbol{x}^{*}-\boldsymbol{x}
$$

and the tracking error, $\boldsymbol{e}_{y}$, is as follows

$$
\begin{aligned}
\boldsymbol{e}_{y} & =\boldsymbol{y}^{*}-\boldsymbol{y} \\
& =\boldsymbol{C} \boldsymbol{x}^{*}-\boldsymbol{C x} \\
& =\boldsymbol{C} \boldsymbol{e}_{x}
\end{aligned}
$$

Taking time derivative of Eq. (5.46) gives

$$
\dot{\boldsymbol{e}}_{x}=\dot{\boldsymbol{x}}^{*}-\dot{\boldsymbol{x}}
$$

Making use of Eqs. (5.25) and (5.43) results in

$$
\dot{\boldsymbol{e}}_{x}=\boldsymbol{A}^{*} \boldsymbol{x}^{*}+\boldsymbol{B}^{*} \boldsymbol{u}^{*}-\boldsymbol{A} \boldsymbol{x}-\boldsymbol{B} \boldsymbol{u}
$$

Adding and subtracting [68] $\boldsymbol{A} \boldsymbol{x}^{*}+\boldsymbol{B} \boldsymbol{u}^{*}$ yields

$$
\dot{\boldsymbol{e}}_{x}=\boldsymbol{A} \boldsymbol{x}^{*}+\boldsymbol{B} \boldsymbol{u}^{*}-\boldsymbol{A} \boldsymbol{x}^{*}-\boldsymbol{B} \boldsymbol{u}^{*}+\boldsymbol{A}^{*} \boldsymbol{x}^{*}+\boldsymbol{B}^{*} \boldsymbol{u}^{*}-\boldsymbol{A x}-\boldsymbol{B u}
$$

Combining some terms and using the definition of the state error from Eq. (5.46) gives

$$
\dot{\boldsymbol{e}}_{x}=\boldsymbol{A} \boldsymbol{e}_{x}-\boldsymbol{B}\left(\boldsymbol{u}-\boldsymbol{u}^{*}\right)+\left(\boldsymbol{A}^{*}-\boldsymbol{A}\right) \boldsymbol{x}^{*}+\left(\boldsymbol{B}^{*}-\boldsymbol{B}\right) \boldsymbol{u}^{*}
$$


Making use of Eqs. (5.21) and (5.45) for $\boldsymbol{u}$ and $\boldsymbol{u}^{*}$, respectively, results in [68]

$$
\begin{aligned}
\dot{\boldsymbol{e}}_{x} & =\boldsymbol{A} \boldsymbol{e}_{x}-\boldsymbol{B}\left[\boldsymbol{K}_{e}(t) \boldsymbol{e}_{y}+\boldsymbol{K}_{x}(t) \boldsymbol{x}_{m}+\boldsymbol{K}_{u}(t) \boldsymbol{u}_{m}\right] \\
& +\boldsymbol{B}\left[\widetilde{\boldsymbol{K}}_{x} \boldsymbol{x}_{m}+\widetilde{\boldsymbol{K}}_{u} \boldsymbol{u}_{m}\right] \\
& +\left(\boldsymbol{A}^{*}-\boldsymbol{A}\right) \boldsymbol{x}^{*}+\left(\boldsymbol{B}^{*}-\boldsymbol{B}\right) \boldsymbol{u}^{*}
\end{aligned}
$$

Adding and subtracting $\boldsymbol{B} \widetilde{\boldsymbol{K}}_{e} \boldsymbol{e}_{y}$ from the above equation yields [68]

$$
\begin{aligned}
\dot{\boldsymbol{e}}_{x} & =\boldsymbol{A} \boldsymbol{e}_{x}-\boldsymbol{B} \widetilde{\boldsymbol{K}}_{e} \boldsymbol{e}_{y}-\boldsymbol{B}\left[\boldsymbol{K}_{e}(t) \boldsymbol{e}_{y}+\boldsymbol{K}_{x}(t) \boldsymbol{x}_{m}+\boldsymbol{K}_{u}(t) \boldsymbol{u}_{m}\right] \\
& +\boldsymbol{B}\left[\widetilde{\boldsymbol{K}}_{x} \boldsymbol{x}_{m}+\widetilde{\boldsymbol{K}}_{u} \boldsymbol{u}_{m}+\widetilde{\boldsymbol{K}}_{e} \boldsymbol{e}_{y}\right] \\
& +\left(\boldsymbol{A}^{*}-\boldsymbol{A}\right) \boldsymbol{x}^{*}+\left(\boldsymbol{B}^{*}-\boldsymbol{B}\right) \boldsymbol{u}^{*}
\end{aligned}
$$

Making use of Eqs. (5.21), (5.22), and (5.47) gives [68]

$$
\begin{aligned}
\dot{\boldsymbol{e}}_{x} & =\left(\boldsymbol{A}-\boldsymbol{B} \widetilde{\boldsymbol{K}}_{e} \boldsymbol{C}\right) \boldsymbol{e}_{x}-\boldsymbol{B}\left(\boldsymbol{K}_{I}(t)-\widetilde{\boldsymbol{K}}\right) \boldsymbol{r} \\
& +\left(\boldsymbol{A}^{*}-\boldsymbol{A}\right) \boldsymbol{x}^{*}+\left(\boldsymbol{B}^{*}-\boldsymbol{B}\right) \boldsymbol{u}^{*}-\boldsymbol{B} \boldsymbol{K}_{p}(t) \boldsymbol{r}
\end{aligned}
$$




\subsection{Stability Analysis}

The following quadratic positive definite Lyapunov function is chosen as seen in various literature $[68,69,72]$.

$$
V=\boldsymbol{e}_{x}^{T} \boldsymbol{P} \boldsymbol{e}_{x}+\operatorname{tr}\left[\left(\boldsymbol{K}_{I}(t)-\widetilde{\boldsymbol{K}}\right) \Gamma^{-1}\left(\boldsymbol{K}_{I}(t)-\widetilde{\boldsymbol{K}}\right)^{T}\right]
$$

Taking the time derivative of Eq. (5.55) along its trajectory gives

$$
\begin{aligned}
\dot{V} & =\boldsymbol{e}_{x}^{T} \dot{\boldsymbol{P}}_{x}+\dot{\boldsymbol{e}}_{x}^{T} \boldsymbol{P} \boldsymbol{e}_{x}+\boldsymbol{e}_{x}^{T} \boldsymbol{P} \dot{\boldsymbol{e}}_{x} \\
& +\operatorname{tr}\left[\dot{\boldsymbol{K}}_{I}(t) \Gamma^{-1}\left(\boldsymbol{K}_{I}(t)-\widetilde{\boldsymbol{K}}\right)^{T}\right] \\
& +\operatorname{tr}\left[\left(\boldsymbol{K}_{I}(t)-\widetilde{\boldsymbol{K}}\right) \Gamma^{-1} \dot{\boldsymbol{K}}_{I}(t)^{T}\right]
\end{aligned}
$$

Making use of Eq. (5.54) and knowing $\dot{\boldsymbol{K}}_{I}=\boldsymbol{e}_{y} \boldsymbol{r}^{T} \Gamma$ yields

$$
\begin{aligned}
\dot{V} & =\boldsymbol{e}_{x}^{T} \dot{\boldsymbol{P}} \boldsymbol{e}_{x}+\boldsymbol{e}_{x}^{T}\left(\boldsymbol{A}-\boldsymbol{B} \widetilde{\boldsymbol{K}}_{e} \boldsymbol{C}\right)^{T} \boldsymbol{P} \boldsymbol{e}_{x}+\boldsymbol{e}_{x}^{T} \boldsymbol{P}\left(\boldsymbol{A}-\boldsymbol{B} \widetilde{\boldsymbol{K}}_{e} \boldsymbol{C}\right) \boldsymbol{e}_{x} \\
& -\boldsymbol{r}^{T}\left(\boldsymbol{K}_{I}(t)-\widetilde{\boldsymbol{K}}\right)^{T} \boldsymbol{B}^{T} \boldsymbol{P} \boldsymbol{e}_{x}-\boldsymbol{e}_{x}^{T} \boldsymbol{P} \boldsymbol{B}\left(\boldsymbol{K}_{I}(t)-\widetilde{\boldsymbol{K}}\right) \boldsymbol{r} \\
& +\left[\left(\boldsymbol{A}^{*}-\boldsymbol{A}\right) \boldsymbol{x}^{*}+\left(\boldsymbol{B}^{*}-\boldsymbol{B}\right) \boldsymbol{u}^{*}\right]^{T} \boldsymbol{P} \boldsymbol{e}_{x}+\boldsymbol{e}_{x}^{T} \boldsymbol{P}\left[\left(\boldsymbol{A}^{*}-\boldsymbol{A}\right) \boldsymbol{x}^{*}+\left(\boldsymbol{B}^{*}-\boldsymbol{B}\right) \boldsymbol{u}^{*}\right] \\
& -\boldsymbol{r}^{T} \boldsymbol{K}_{p}(t)^{T} \boldsymbol{B}^{T} \boldsymbol{P} \boldsymbol{e}_{x}-\boldsymbol{e}_{x}^{T} \boldsymbol{P} \boldsymbol{B} \boldsymbol{K}_{p}(t) \boldsymbol{r} \\
& +\operatorname{tr}\left[\boldsymbol{e}_{y} \boldsymbol{r}^{T} \Gamma \Gamma^{-1}\left(\boldsymbol{K}_{I}(t)-\widetilde{\boldsymbol{K}}\right)^{T}\right]+\operatorname{tr}\left[\left(\boldsymbol{K}_{I}(t)-\widetilde{\boldsymbol{K}}\right) \Gamma^{-1} \Gamma \boldsymbol{r} \boldsymbol{e}_{y}^{T}\right]
\end{aligned}
$$

Using Eqs. (5.27) and (5.28), that is the ASP conditions, Eq. (5.47) for $\boldsymbol{e}_{y}=\boldsymbol{C} \boldsymbol{e}_{x}$, and $\boldsymbol{x}^{T} \boldsymbol{y}=\boldsymbol{y}^{T} \boldsymbol{x}$ property [68] results in

$$
\begin{aligned}
\dot{V}= & -\boldsymbol{e}_{x}^{T} \boldsymbol{Q} \boldsymbol{e}_{x} \\
& -\boldsymbol{r}^{T}\left(\boldsymbol{K}_{I}(t)-\widetilde{\boldsymbol{K}}\right)^{T} \boldsymbol{C} \boldsymbol{e}_{x}-\boldsymbol{e}_{x}^{T} \boldsymbol{C}^{T}\left(\boldsymbol{K}_{I}(t)-\widetilde{\boldsymbol{K}}\right) \boldsymbol{r} \\
& +\left[\left(\boldsymbol{A}^{*}-\boldsymbol{A}\right) \boldsymbol{x}^{*}+\left(\boldsymbol{B}^{*}-\boldsymbol{B}\right) \boldsymbol{u}^{*}\right]^{T} \boldsymbol{P} \boldsymbol{e}_{x}+\boldsymbol{e}_{x}^{T} \boldsymbol{P}\left[\left(\boldsymbol{A}^{*}-\boldsymbol{A}\right) \boldsymbol{x}^{*}+\left(\boldsymbol{B}^{*}-\boldsymbol{B}\right) \boldsymbol{u}^{*}\right] \\
& -\boldsymbol{e}_{x}^{T} \boldsymbol{C}^{T} \boldsymbol{K}_{p}(t) \boldsymbol{r}-\boldsymbol{e}_{x}^{T} \boldsymbol{C}^{T} \boldsymbol{K}_{p}(t) \boldsymbol{r} \\
& +\operatorname{tr}\left[\left(\boldsymbol{K}_{I}(t)-\widetilde{\boldsymbol{K}}\right) \boldsymbol{r} \boldsymbol{e}_{x}^{T} \boldsymbol{C}^{T}\right]+\operatorname{tr}\left[\boldsymbol{C} \boldsymbol{e}_{x} \boldsymbol{r}^{T}\left(\boldsymbol{K}_{I}(t)-\widetilde{\boldsymbol{K}}\right)^{T}\right]
\end{aligned}
$$


By using $\operatorname{tr}(\boldsymbol{A B})=\operatorname{tr}(\boldsymbol{B} \boldsymbol{A})$ and $\operatorname{tr}\left(\boldsymbol{x}^{T} \boldsymbol{y}\right)=\boldsymbol{x}^{T} \boldsymbol{y}$ properties [68], $\dot{V}$ simplifies to

$$
\begin{aligned}
\dot{V}= & -\boldsymbol{e}_{x}^{T} \boldsymbol{Q} \boldsymbol{e}_{x} \\
& -\boldsymbol{r}^{T}\left(\boldsymbol{K}_{I}(t)-\widetilde{\boldsymbol{K}}\right)^{T} \boldsymbol{C} \boldsymbol{e}_{x}-\boldsymbol{e}_{x}^{T} \boldsymbol{C}^{T}\left(\boldsymbol{K}_{I}(t)-\widetilde{\boldsymbol{K}}\right) \boldsymbol{r} \\
& +\left[\left(\boldsymbol{A}^{*}-\boldsymbol{A}\right) \boldsymbol{x}^{*}+\left(\boldsymbol{B}^{*}-\boldsymbol{B}\right) \boldsymbol{u}^{*}\right]^{T} \boldsymbol{P} \boldsymbol{e}_{x}+\boldsymbol{e}_{x}^{T} \boldsymbol{P}\left[\left(\boldsymbol{A}^{*}-\boldsymbol{A}\right) \boldsymbol{x}^{*}+\left(\boldsymbol{B}^{*}-\boldsymbol{B}\right) \boldsymbol{u}^{*}\right] \\
& -2 \boldsymbol{e}_{x}^{T} \boldsymbol{C}^{T} \boldsymbol{K}_{p}(t) \boldsymbol{r} \\
& +\boldsymbol{e}_{x}^{T} \boldsymbol{C}^{T}\left(\boldsymbol{K}_{I}(t)-\widetilde{\boldsymbol{K}}\right) \boldsymbol{r}+\boldsymbol{r}^{T}\left(\boldsymbol{K}_{I}(t)-\widetilde{\boldsymbol{K}}\right)^{T} \boldsymbol{C} \boldsymbol{e}_{x}
\end{aligned}
$$

Further simplification gives

$$
\begin{aligned}
\dot{V}= & -\boldsymbol{e}_{x}^{T} \boldsymbol{Q} \boldsymbol{e}_{x} \\
& +\left[\left(\boldsymbol{A}^{*}-\boldsymbol{A}\right) \boldsymbol{x}^{*}+\left(\boldsymbol{B}^{*}-\boldsymbol{B}\right) \boldsymbol{u}^{*}\right]^{T} \boldsymbol{P} \boldsymbol{e}_{x}+\boldsymbol{e}_{x}^{T} \boldsymbol{P}\left[\left(\boldsymbol{A}^{*}-\boldsymbol{A}\right) \boldsymbol{x}^{*}+\left(\boldsymbol{B}^{*}-\boldsymbol{B}\right) \boldsymbol{u}^{*}\right] \\
& -2 \boldsymbol{e}_{x}^{T} \boldsymbol{C}^{T} \boldsymbol{K}_{p}(t) \boldsymbol{r}
\end{aligned}
$$

Assuming that systems parameters vary slowly with time compared to control dynamics, meaning that $\boldsymbol{A}^{*}=\boldsymbol{A}$ and $\boldsymbol{B}^{*}=\boldsymbol{B}$ [68], gives the following

$$
\dot{V}=-\boldsymbol{e}_{x}^{T} \boldsymbol{Q} \boldsymbol{e}_{x}-2 \boldsymbol{e}_{x}^{T} \boldsymbol{C}^{T} \boldsymbol{K}_{p}(t) \boldsymbol{r}
$$

The derivative of the Lyapunov function is negative definite in $\boldsymbol{e}_{x}$ and negative semi-definite in the state-gain space $\left\{\boldsymbol{e}_{x}, \boldsymbol{K}_{I}(t)\right\}$. This means that all values are bounded $[68,69]$.

Furthermore, by using LaSalle's invariance principle for non-autonomous systems, the domain $\left\{\boldsymbol{e}_{x}, \boldsymbol{K}_{I}(t)\right\}$ reaches 0 when $\boldsymbol{e}_{x} \equiv 0$. This will imply that $\boldsymbol{e}_{y}=0$ from Eq. (5.47) and thus the system is asymptotically stable. 


\section{Chapter 6}

\section{Weighted Pseudo Inverse}

This chapter highlights a weighted pseudo inverse optimization based algorithm for control allocation (CA). First, the CA concept and its integration with the controller are explained in Sec. 6.1: Introduction to Control Allocation. Section 6.2: Problem Formulation defines the state space representation for systems with faults. This is followed by Sec. 6.3: Optimization Problem, where the optimization problem for weighted pseudo inverse and its solution are presented.

\subsection{Introduction to Control Allocation}

CA algorithms are often based on optimization techniques. CA offers many advantages. Various issues such as input saturation and rate constraints, actuator and effector fault tolerance, and secondary objectives such as power efficiency and wear-and-tear minimization can be handled with CA [16]. For example, in order to improve the performance of a system such as precision, response time, and load sharing, a hierarchical actuator can be implemented [16,73]. This is done by combining multiple small actuators and by arranging them in a specified manner to improve the performance of the system and to meet the control objectives [73].

Another use of CA is for systems that allow actuators to be used for multiple control systems with different objectives [16]. An example of this would be using the same set of actuators to control the position and attitude of a spacecraft. Another usage of CA is for systems that are equipped with redundant actuators. This is especially beneficial for maintaining the required performance of the system during faults [16].

There are two steps to CA. This is illustrated in Fig. 6.1. The first one is the high-level control algorithm that generates the control input, which is referred to as the virtual control input in CA, denoted by $\boldsymbol{u}_{d}$. The dimension of the virtual control input matrix often equals the number of degrees of the freedom of the system [16], which is three for SFF in this thesis. For the CA part of this thesis, SAC will be 
used as the baseline controller.

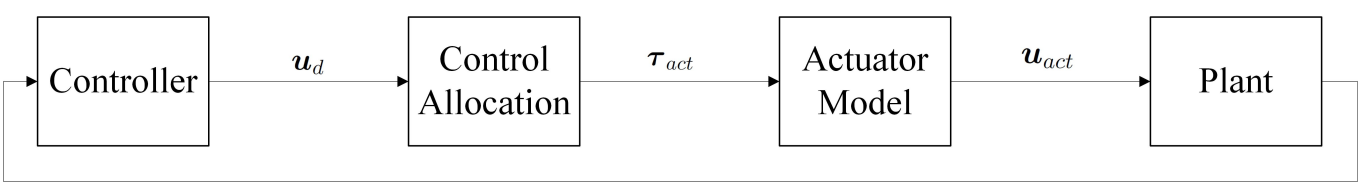

Figure 6.1: Overall Control Allocation Strategy

The second part for CA is the CA itself, which maps the virtual control input that comes from the controller to the individual actuators, denoted by $\boldsymbol{\tau}_{\text {act }}$. The total forces or moments in the individual actuators should add up to the required control input given by the control law [16].

\subsection{Problem Formulation}

For a LTI system with actuator faults, the state space representation is given by [74]

$$
\begin{aligned}
\dot{\boldsymbol{x}} & =\boldsymbol{A} \boldsymbol{x}+\boldsymbol{D} \boldsymbol{E} \boldsymbol{\tau}_{a c t} \\
& =\boldsymbol{A} \boldsymbol{x}+\boldsymbol{u}_{a c t}
\end{aligned}
$$

with $\boldsymbol{D}$ being the actuator distribution matrix, $\boldsymbol{E}$ a diagonal matrix that models the effectiveness level of the actuators, and $\boldsymbol{\tau}_{a c t}$, the control input that is distributed to the individual actuators.

More specifically, the effectiveness matrix is defined as $\boldsymbol{E}=\operatorname{diag}\left[e_{1}(t), e_{2}(t), \ldots, e_{n}(t)\right]$ $\in \mathbb{R}^{n \times n}$. A value of 0 for $e_{i}$ indicates a complete failure of the $i^{t h}$ actuator, a value of 1 means that the actuator is healthy, and $0<e_{i}<1$ denotes partial loss of effectiveness of the $i^{t h}$ actuator .

Most CA algorithms assume a linear effector model as such [16]

$$
\boldsymbol{u}_{d}=\boldsymbol{D} \boldsymbol{\tau}_{\text {act }}
$$

where $\boldsymbol{u}_{d} \in \mathbb{R}^{m \times 1}, \boldsymbol{D} \in \mathbb{R}^{m \times n}$, and $\boldsymbol{\tau}_{\text {act }} \in \mathbb{R}^{n \times 1}$.

The matrix $\boldsymbol{D}$ is not a square matrix. It has a full row rank $(n<m)$ and a nontrivial null space. This means that an infinite number of $\boldsymbol{\tau}_{\text {act }}$ exist that can satisfy Eq. (6.2) for a given $\boldsymbol{u}_{d}$ [16].

The control signal sent to the actuators can be obtained by

$$
\boldsymbol{\tau}_{a c t}=\boldsymbol{D}^{+} \boldsymbol{u}_{d}
$$


where $\boldsymbol{D}^{+}$is the pseudo inverse of the matrix $\boldsymbol{D}$. The matrix $\boldsymbol{D}^{+}$allocates the virtual control signal to the individual actuators [74]. The goal is to find a solution for $\boldsymbol{D}^{+}$to optimally distribute the control signal among the actuators.

The estimate of the faults, denoted by $\hat{\boldsymbol{E}}$, should satisfy the following [38]

$$
\boldsymbol{E}=(\boldsymbol{I}-\boldsymbol{\Delta}) \hat{\boldsymbol{E}}
$$

where $\boldsymbol{\Delta}$ is the level of imperfection in the estimation, which can be described as $\boldsymbol{\Delta}=\operatorname{diag}\left[\delta_{1}(t), \delta_{2}(t), \ldots, \delta_{n}(t)\right]$ and $\hat{\boldsymbol{E}} \in \xi$ satisfies $\xi=\left\{\left(\hat{e}_{1}, \ldots, \hat{e}_{n}\right) \in[0,1]^{n}:\right.$ $\left.\operatorname{det}\left(\boldsymbol{D} \hat{\boldsymbol{E}}^{3} \boldsymbol{D}^{T}\right) \neq 0\right\}$.

For up to $m-n$ failures, that is $\hat{e}_{i}=0$, the $\operatorname{det}\left(\boldsymbol{D} \hat{\boldsymbol{E}}^{3} \boldsymbol{D}^{T}\right) \neq 0$. If more than $m-n$ actuators fail, then $\operatorname{det}\left(\boldsymbol{D} \hat{\boldsymbol{E}}^{3} \boldsymbol{D}^{T}\right)=0$ and the system becomes unstable as the $\operatorname{rank} \hat{E}<n$. Therefore, the statement should be made that only $m-n$ actuators can fail, while others lose their effectiveness [39]. For SFF, this translates into one actuator completely failing while the other three experience partial loss of effectiveness. 


\subsection{Optimization Problem}

For a weighted pseudo inverse based CA method, the optimization problem is given by [39]

$$
\begin{aligned}
\min & \boldsymbol{\tau}_{a c t}^{T} \hat{\boldsymbol{E}}^{-1} \boldsymbol{\tau}_{a c t} \\
\text { subject to } & \boldsymbol{D} \hat{\boldsymbol{E}} \boldsymbol{\tau}_{a c t}=\boldsymbol{u}_{d}
\end{aligned}
$$

where the objective is to minimize the weighted 2-norm of the actuation.

The solution to the optimization problem is

$$
\boldsymbol{\tau}_{a c t}=\hat{\boldsymbol{E}}^{2} \boldsymbol{D}^{T}\left(\boldsymbol{D} \hat{\boldsymbol{E}}^{3} \boldsymbol{D}^{T}\right)^{-1} \boldsymbol{u}_{d}
$$

For this weighted pseudo inverse CA problem, as $\hat{e}_{i}(t) \rightarrow 0$, indicating a fault, the corresponding $u_{i}$ weighs heavily since $1 / \hat{e}_{i}(t)$ becomes large. Thus, $u_{i}$ is reallocated to other healthier actuators [38].

Proof: First the Lagrangian is defined as [75]

$$
\boldsymbol{L}\left(\boldsymbol{\tau}_{a c t}, \lambda\right)=\frac{1}{2} \boldsymbol{\tau}_{a c t}^{T} \hat{\boldsymbol{E}}^{-1} \boldsymbol{\tau}_{a c t}+\lambda\left(\boldsymbol{D} \hat{\boldsymbol{E}} \boldsymbol{\tau}_{a c t}-\boldsymbol{u}_{d}\right)
$$

where $\lambda$ is the Lagrange multiplier.

Taking the partial derivative of Eq. (6.7) with respect to $\boldsymbol{\tau}_{a c t}$ and $\lambda$ and equating them to zero yields [75]

$$
\begin{gathered}
\frac{\partial \boldsymbol{L}}{\partial \boldsymbol{\tau}_{a c t}}=\boldsymbol{\tau}_{a c t}^{T} \hat{\boldsymbol{E}}^{-1}+\lambda \boldsymbol{D} \hat{\boldsymbol{E}}=0 \\
\frac{\partial \boldsymbol{L}}{\partial \lambda}=\boldsymbol{D} \hat{\boldsymbol{E}} \boldsymbol{\tau}_{a c t}-\boldsymbol{u}_{d}=0
\end{gathered}
$$

Post multiplying Eq. (6.8) by $\hat{\boldsymbol{E}}$ results in [75]

$$
\begin{aligned}
\boldsymbol{\tau}_{a c t}^{T} & =-\lambda \boldsymbol{D} \hat{\boldsymbol{E}} \hat{\boldsymbol{E}} \\
& =-\lambda \boldsymbol{D} \hat{\boldsymbol{E}}^{2}
\end{aligned}
$$

which can then be solved for $\boldsymbol{\tau}_{\text {act }}$ by taking the transpose of both sides as such

$$
\boldsymbol{\tau}_{a c t}=-\hat{\boldsymbol{E}}^{2} \boldsymbol{D}^{T} \lambda
$$


Substitute Eq. (6.11) into Eq. (6.9) and solving for $\boldsymbol{u}_{d}$ gives

$$
\begin{aligned}
\boldsymbol{u}_{d} & =-\boldsymbol{D} \hat{\boldsymbol{E}}\left(\hat{\boldsymbol{E}}^{2} \boldsymbol{D}^{T} \lambda\right) \\
& =-\boldsymbol{D} \hat{\boldsymbol{E}}^{3} \boldsymbol{D}^{T} \lambda
\end{aligned}
$$

Equation (6.12) can be solved for $\lambda$ as such

$$
\lambda=-\left(\boldsymbol{D} \hat{\boldsymbol{E}}^{3} \boldsymbol{D}^{T}\right)^{-1} \boldsymbol{u}_{d}
$$

assuming that $\left\{\operatorname{det}\left(\boldsymbol{D} \hat{\boldsymbol{E}}^{3} \boldsymbol{D}^{T}\right) \neq 0\right\}$.

The solution for $\boldsymbol{\tau}_{\text {act }}$ is obtained by substituting Eq. (6.13) into Eq. (6.11) as follows

$$
\begin{aligned}
\boldsymbol{\tau}_{a c t} & =-\hat{\boldsymbol{E}}^{2} \boldsymbol{D}^{T}\left[-\left(\boldsymbol{D} \hat{\boldsymbol{E}}^{3} \boldsymbol{D}^{T}\right)^{-1} \boldsymbol{u}_{d}\right] \\
& =\hat{\boldsymbol{E}}^{2} \boldsymbol{D}^{T}\left(\boldsymbol{D} \hat{\boldsymbol{E}}^{3} \boldsymbol{D}^{T}\right)^{-1} \boldsymbol{u}_{d}
\end{aligned}
$$

The solution to the optimization problem can then be written more compactly as

$$
\boldsymbol{\tau}_{\text {act }}=\boldsymbol{D}^{+} \boldsymbol{u}_{d}
$$

where $\boldsymbol{D}^{+}=\hat{\boldsymbol{E}}^{2} \boldsymbol{D}^{T}\left(\boldsymbol{D} \hat{\boldsymbol{E}}^{3} \boldsymbol{D}^{T}\right)^{-1}$.

Therefore, the state space representation can be rewritten as

$$
\begin{aligned}
\dot{\boldsymbol{x}} & =\boldsymbol{A} \boldsymbol{x}+\boldsymbol{D} \boldsymbol{E} \boldsymbol{D}^{+} \boldsymbol{u}_{d} \\
\dot{\boldsymbol{x}} & =\boldsymbol{A} \boldsymbol{x}+\boldsymbol{D} \boldsymbol{E} \boldsymbol{\tau}_{a c t} \\
& =\boldsymbol{A} \boldsymbol{x}+\boldsymbol{u}_{a c t}
\end{aligned}
$$

which is graphically shown in Fig. 6.2.

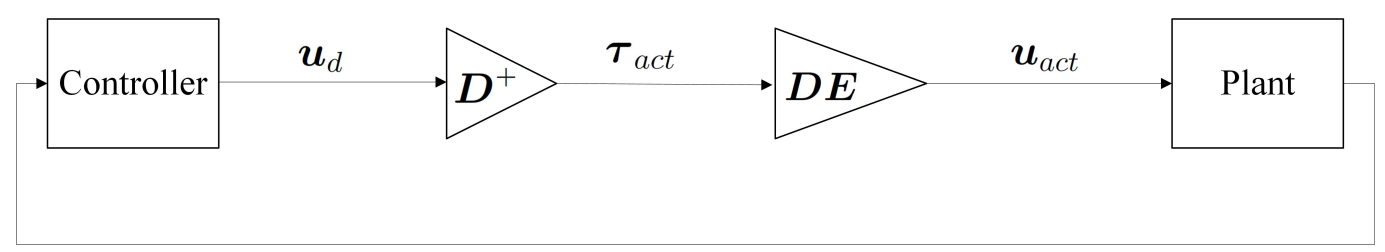

Figure 6.2: Weighted Pseudo Inverse 


\section{Chapter 7}

\section{Quadratic Programming}

This chapter highlights constrained quadratic programming (QP) control allocation. Numerical solutions of optimization problems with an $l_{2}$ norm are generally called QP. More spefically, this chapter presents a closed constrained optimal allocation scheme based on quadratic programming algorithm.

Among the few studies of CLCA, only a few have addressed the stability proof. For example in [76], the open and closed loop performance of $16 \mathrm{CA}$ schemes is presented; however, the stability proof is not discussed. Zhang and Chen present a closed loop control allocation for satellite precision pointing with the stability proof. Compared to the original cascaded generalized inverse method, no steadystate allocation error was observed given the uncertainty of the control effectiveness matrix with the closed loop allocation [77]. Therefore, it can be concluded that the a closed loop control allocation improves the performance of the system.

In view of the above, first a quadratic programming algorithm is used to obtain an open-loop control allocation solution, where actuator faults are considered. Then a closed-loop control allocation scheme is designed.

The rest of this chapter is as follows: Section 7.1: Control Allocation Problem introduces the QP optimization problem. A detailed procedure for obtaining the

solution to the CA optimization problem is given in Sec. 7.2: Open Loop Control Allocation. This is followed by Sec. 7.3: Closed Loop Control Allocation, where the closed looped CA as well as its stability proof are presented. A modified CLCA based on the work in [43] is presented in Sec. 7.4: Modified Closed Loop Control Allocation. 


\subsection{Control Allocation Problem}

The CA algorithm can be formulated as a constrained quadratic programming problem as such

$$
\begin{array}{cl}
J=\underset{\tau(t) \in \Omega_{0}}{\arg \min } & \left\{\left\|\boldsymbol{W}_{0} \boldsymbol{\tau}_{a c t}(t)\right\|^{2}\right. \\
& +\left\|\boldsymbol{W}_{1}\left[\boldsymbol{\tau}_{\text {act }}(t)-\boldsymbol{\tau}_{d}(t)\right]\right\|^{2} \\
& \left.+\left\|\boldsymbol{W}_{2}\left[\boldsymbol{\tau}_{\text {act }}(t)-\boldsymbol{\tau}_{a c t}(t-T)\right]\right\|^{2}\right\} \\
\text { subject to } \quad \boldsymbol{u}_{d}(t)=\boldsymbol{D} \hat{\boldsymbol{E}} \boldsymbol{\tau}_{a c t}(t) & \\
\boldsymbol{\Omega}_{0}=\underset{\underline{\tau}(t) \leqslant \tau_{a c t}(t) \leqslant \bar{\tau}(t)}{\arg \min } & \left\|\boldsymbol{u}_{d}(t)-\boldsymbol{D} \hat{\boldsymbol{E}} \boldsymbol{\tau}_{a c t}(t)\right\|
\end{array}
$$

where $\boldsymbol{W}_{0}, \boldsymbol{W}_{1}$, and $\boldsymbol{W}_{2}$ are diagonal positive definite weighing matrices, $\boldsymbol{u}_{d}$ and $\boldsymbol{\tau}_{d}$ are the desired control torques and actuator torques, $\boldsymbol{u}_{a c t}$ and $\boldsymbol{\tau}_{\text {act }}$ are the actual control torques and actuator torques, $\boldsymbol{D}$ is the actuator configuration matrix, $\hat{\boldsymbol{E}}$ is the estimate of the actuators' faults, and $T$ is the sampling time.

If there are no constrains or actuator misalignment, then $\boldsymbol{u}_{d}(t)=\boldsymbol{D} \hat{\boldsymbol{E}} \boldsymbol{\tau}_{\text {act }}(t)$; since that is not the case, the goal is to minimize the control error $\left\|\boldsymbol{u}_{d}(t)-\boldsymbol{D} \hat{\boldsymbol{E}} \boldsymbol{\tau}_{a c t}(t)\right\|$.

In Eq. (7.1), a large $\boldsymbol{W}_{0}$ contributes to energy consumption minimization, a large $\boldsymbol{W}_{1}$ allows for a fast convergence of the control torque to the desired one, and $\boldsymbol{W}_{2}$ is selected such that a smooth actuator response can be achieved [41]. More specifically, the matrix $\boldsymbol{W}_{0}$ is given by

$$
\boldsymbol{W}_{0}=\left(\hat{\boldsymbol{E}}+\delta_{e} \boldsymbol{I}\right)^{-1}
$$

where $\hat{\boldsymbol{E}}$ is the estimate of the actuator faults, as previously explained in Sec. 6.2, and $\delta_{e}$ is a small number, so that the inverse can be calculated. 


\subsection{Open Loop Control Allocation}

The solution to the optimization problem in Eq. (7.1) is

$$
\boldsymbol{\tau}_{a c t}=\boldsymbol{H} \boldsymbol{\tau}_{d}(t)+\boldsymbol{F} \boldsymbol{\tau}_{a c t}(t-T)+\boldsymbol{G} \boldsymbol{u}_{d}(t)
$$

where

$$
\begin{aligned}
\boldsymbol{H} & =(\boldsymbol{I}-\boldsymbol{G} \boldsymbol{D} \hat{\boldsymbol{E}}) \boldsymbol{W}^{-2} \boldsymbol{W}_{1}^{2} \\
\boldsymbol{F} & =(\boldsymbol{I}-\boldsymbol{G} \boldsymbol{D} \hat{\boldsymbol{E}}) \boldsymbol{W}^{-2} \boldsymbol{W}_{2}^{2} \\
\boldsymbol{G} & =\boldsymbol{W}^{-1}\left(\boldsymbol{D} \hat{\boldsymbol{E}} \boldsymbol{W}^{-1}\right)^{+}
\end{aligned}
$$

Proof: The constrained quadratic programming problem can be rewritten as

$$
\underset{\tau(t) \in \Omega_{0}}{\arg \min }\left\|\boldsymbol{W}\left(\boldsymbol{\tau}_{a c t}-\boldsymbol{x}_{0}\right)\right\|
$$

where

$$
\boldsymbol{W}=\left(\left(\left(\hat{\boldsymbol{E}}+\delta_{e} \boldsymbol{I}\right)^{-1}\right)^{2}+\boldsymbol{W}_{1}^{2}+\boldsymbol{W}_{2}^{2}\right)^{\frac{1}{2}}
$$

and

$$
\boldsymbol{x}_{0}=\boldsymbol{W}^{-2}\left(\boldsymbol{W}_{1}^{2} \boldsymbol{\tau}_{d}+\boldsymbol{W}_{2}^{2} \boldsymbol{\tau}_{a c t}(t-T)\right)
$$

Equation (7.7) has the same minimizing argument as Eq. (7.1).

Lemma 1: For a matrix $\boldsymbol{A} \in \mathbb{R}^{m \times n}$, where $m<n$, with rank $m$, the minimum norm problem is expressed as [78]

$$
\begin{aligned}
\min _{x} & \|\boldsymbol{x}\| \\
\text { subject to } & \boldsymbol{A} \boldsymbol{x}=\boldsymbol{y}
\end{aligned}
$$

and it has the solution

$$
\boldsymbol{x}=\boldsymbol{A}^{+} \boldsymbol{y}
$$

where $\boldsymbol{A}^{+}$is the pseudo inverse of $\boldsymbol{A}, \boldsymbol{A}^{+}=\boldsymbol{A}^{T}\left(\boldsymbol{A} \boldsymbol{A}^{T}\right)^{-1}$.

Lemma 2: The least squares problem is given by [78]

$$
\begin{aligned}
\min _{x} & \left\|\boldsymbol{W}\left(\boldsymbol{x}-\boldsymbol{x}_{0}\right)\right\| \\
\text { subject to } & \boldsymbol{A} \boldsymbol{x}=\boldsymbol{y}
\end{aligned}
$$


In order to solve the least squares problem, let $\boldsymbol{e}=\boldsymbol{W}\left(\boldsymbol{x}-\boldsymbol{x}_{0}\right)$, where $\boldsymbol{W}$ is nonsingular [78]. Rearranging this for $\boldsymbol{x}$ gives

$$
\boldsymbol{x}=\boldsymbol{x}_{0}+\boldsymbol{W}^{-1} \boldsymbol{e}
$$

The optimization problem can then be reformulated as [78]

$$
\begin{aligned}
\min _{e} & \|\boldsymbol{e}\| \\
\text { subject to } & \boldsymbol{A}\left(\boldsymbol{x}_{0}+\boldsymbol{W}^{-1} \boldsymbol{e}\right)=\boldsymbol{y}
\end{aligned}
$$

and it has the solution

$$
\boldsymbol{e}=\left(\boldsymbol{A} \boldsymbol{W}^{-1}\right)^{+}\left(\boldsymbol{y}-\boldsymbol{A} \boldsymbol{x}_{0}\right)
$$

Substituting the above into Eq. (7.13) yields [78]

$$
\boldsymbol{x}=\boldsymbol{x}_{0}+\boldsymbol{W}^{-1}\left[\left(\boldsymbol{A} \boldsymbol{W}^{-1}\right)^{+}\left(\boldsymbol{y}-\boldsymbol{A} \boldsymbol{x}_{0}\right)\right]
$$

which can be rearranged to

$$
\boldsymbol{x}=\left[\boldsymbol{I}-\boldsymbol{W}^{-1}\left(\boldsymbol{A} \boldsymbol{W}^{-1}\right)^{+} \boldsymbol{A}\right] \boldsymbol{x}_{0}+\boldsymbol{W}^{-1}\left(\boldsymbol{A} \boldsymbol{W}^{-1}\right)^{+} \boldsymbol{y}
$$

The solution can be rewritten more compactly as [78]

$$
\boldsymbol{x}=\boldsymbol{M} \boldsymbol{x}_{0}+\boldsymbol{G} \boldsymbol{y}
$$

where

$$
\boldsymbol{G}=\boldsymbol{W}^{-1}\left(\boldsymbol{A} \boldsymbol{W}^{-1}\right)^{+}
$$

and

$$
M=I-G A
$$

Equation (7.7) is similar to Eq. (7.12). Substituting in the appropriate matrices such that $\boldsymbol{x}=\boldsymbol{\tau}_{a c t}, \boldsymbol{y}=\boldsymbol{u}_{d}$, and $\boldsymbol{A}=\boldsymbol{D} \hat{\boldsymbol{E}}$ and making use of Eq. (7.9) gives

$$
\boldsymbol{\tau}_{a c t}=\boldsymbol{M}\left[\boldsymbol{W}^{-2}\left\{\boldsymbol{W}_{1}^{2} \boldsymbol{\tau}_{d}(t)+\boldsymbol{W}_{2}^{2} \boldsymbol{\tau}_{a c t}(t-T)\right\}\right]+\boldsymbol{G} \boldsymbol{u}_{d}(t)
$$

which can be expanded as 


$$
\begin{aligned}
\boldsymbol{\tau}_{a c t} & =\left[(\boldsymbol{I}-\boldsymbol{G} \boldsymbol{D} \hat{\boldsymbol{E}}) \boldsymbol{W}^{-2} \boldsymbol{W}_{1}^{2}\right] \boldsymbol{\tau}_{d}(t) \\
& +\left[(\boldsymbol{I}-\boldsymbol{G} \boldsymbol{D} \hat{\boldsymbol{E}}) \boldsymbol{W}^{-2} \boldsymbol{W}_{2}^{2}\right] \boldsymbol{\tau}_{a c t}(t-T) \\
& +\boldsymbol{W}^{-1}\left(\boldsymbol{D} \hat{\boldsymbol{E}} \boldsymbol{W}^{-1}\right)^{+} \boldsymbol{u}_{d}(t)
\end{aligned}
$$

Equation (7.22) is the solution of the CA problem in Eq. (7.1). 


\subsection{Closed Loop Control Allocation}

The closed-loop control allocation (CLCA) system can be described as

$$
\begin{aligned}
{\left[\boldsymbol{I}-z^{-1}(\boldsymbol{I}-\boldsymbol{L})\right] \boldsymbol{U}_{a c t}(z) } & =\left[\boldsymbol{L}+\boldsymbol{D} \hat{\boldsymbol{E}} \boldsymbol{G}-z^{-1} \boldsymbol{D} \hat{\boldsymbol{E}} \boldsymbol{G}\right] \boldsymbol{U}_{d}(z) \\
& +\boldsymbol{D} \hat{\boldsymbol{E}} \boldsymbol{F} z^{-1}\left(1-z^{-1}\right) \boldsymbol{\Gamma}_{a c t}(z)
\end{aligned}
$$

where the eigenvalues of $\boldsymbol{L}$ are in the domain $(0,1)$, guaranteeing that the system is asymptotically stable. The CLCA is shown in Fig. 7.1.

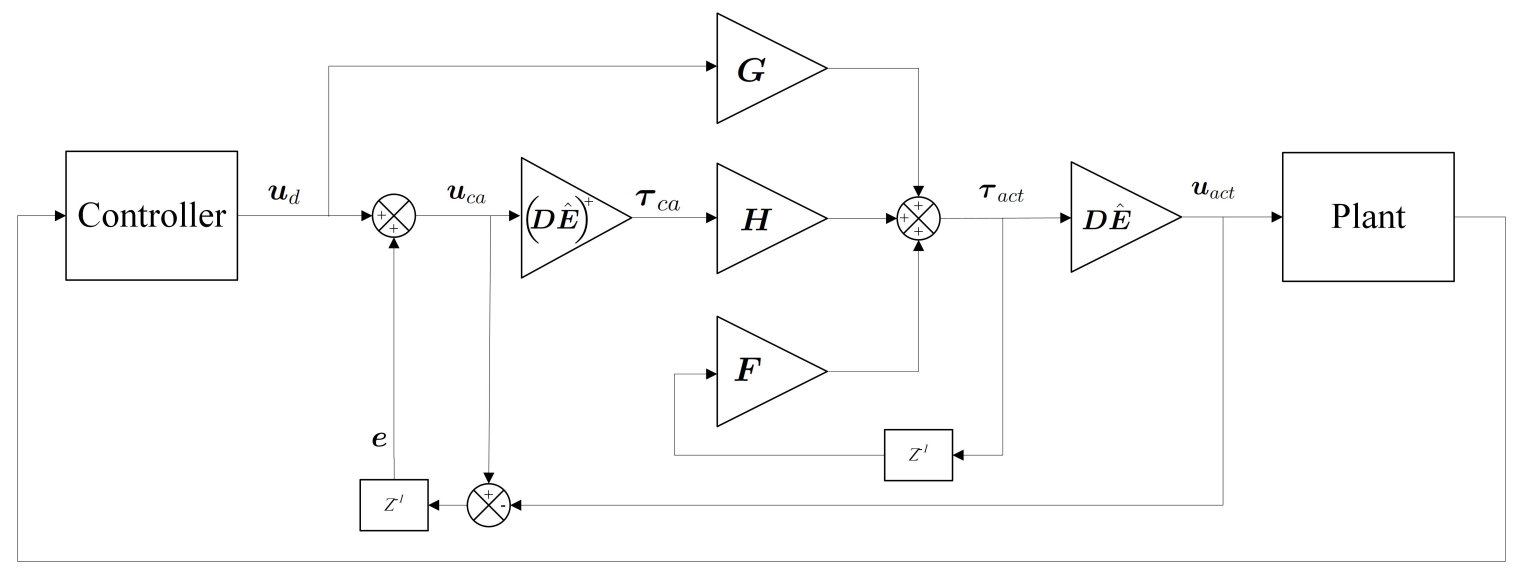

Figure 7.1: Closed Loop Control Allocation Diagram

Proof: The closed loop equation for $\mathrm{CA}$ is given by the following discrete-time model as such [41]

$$
\boldsymbol{\tau}_{a c t}(k)=\boldsymbol{H} \boldsymbol{\tau}_{c a}(k)+\boldsymbol{F} \boldsymbol{\tau}_{a c t}(k-1)+\boldsymbol{G} \boldsymbol{u}_{d}(k)
$$

where

$$
\boldsymbol{\tau}_{c a}(k)=(\boldsymbol{D} \hat{\boldsymbol{E}})^{+} \boldsymbol{u}_{c a}(k)
$$

and

$$
\boldsymbol{u}_{a c t}(k)=\boldsymbol{D} \hat{\boldsymbol{E}} \boldsymbol{\tau}_{a c t}(k)
$$

Substituting Eqs. (7.25) and (7.26) into Eq. (7.24) yields

$$
\boldsymbol{u}_{a c t}(k)=\boldsymbol{D} \hat{\boldsymbol{E}} \boldsymbol{H}(\boldsymbol{D} \hat{\boldsymbol{E}})^{+} \boldsymbol{u}_{c a}(k)+\boldsymbol{D} \hat{\boldsymbol{E}} \boldsymbol{F} \boldsymbol{\tau}_{a c t}(k-1)+\boldsymbol{D} \hat{\boldsymbol{E}} \boldsymbol{G} \boldsymbol{u}_{d}(k)
$$

Equation (7.27) can be rearranged as follows

$$
\boldsymbol{D} \hat{\boldsymbol{E}} \boldsymbol{H}(\boldsymbol{D} \hat{\boldsymbol{E}})^{+} \boldsymbol{u}_{c a}(k)=\boldsymbol{u}_{a c t}(k)-\boldsymbol{D} \hat{\boldsymbol{E}} \boldsymbol{F} \boldsymbol{\tau}_{a c t}(k-1)-\boldsymbol{D} \hat{\boldsymbol{E}} \boldsymbol{G} \boldsymbol{u}_{d}(k)
$$


The CA error for the closed loop is given by

$$
\boldsymbol{e}(k)=\boldsymbol{u}_{c a}(k)-\boldsymbol{u}_{a c t}(k)
$$

and $\boldsymbol{u}_{c a}(k)$ can be expressed as

$$
\boldsymbol{u}_{c a}(k)=\boldsymbol{u}_{d}(k)+\boldsymbol{e}(k-1)
$$

Substituting in the error from Eq. (7.29) into Eq. (7.30) yields [41]

$$
\boldsymbol{u}_{c a}(k)=\boldsymbol{u}_{d}(k)+\boldsymbol{u}_{c a}(k-1)-\boldsymbol{u}_{a c t}(k-1)
$$

Multiply Eq. (7.31) by $\boldsymbol{D} \hat{\boldsymbol{E}} \boldsymbol{H}(\boldsymbol{D} \hat{\boldsymbol{E}})^{+}$gives

$$
\begin{aligned}
\boldsymbol{D} \hat{\boldsymbol{E}} \boldsymbol{H}(\boldsymbol{D} \hat{\boldsymbol{E}})^{+} \boldsymbol{u}_{c a}(k) & =\boldsymbol{D} \hat{\boldsymbol{E}} \boldsymbol{H}(\boldsymbol{D} \hat{\boldsymbol{E}})^{+} \boldsymbol{u}_{d}(k) \\
& +\boldsymbol{D} \hat{\boldsymbol{E}} \boldsymbol{H}(\boldsymbol{D} \hat{\boldsymbol{E}})^{+} \boldsymbol{u}_{c a}(k-1) \\
& -\boldsymbol{D} \hat{\boldsymbol{E}} \boldsymbol{H}(\boldsymbol{D} \hat{\boldsymbol{E}})^{+} \boldsymbol{u}_{a c t}(k-1)
\end{aligned}
$$

Letting $\boldsymbol{D} \hat{\boldsymbol{E}} \boldsymbol{H}(\boldsymbol{D} \hat{\boldsymbol{E}})^{+}=\boldsymbol{L}$ and substituting in Eq. (7.28) for $\boldsymbol{L} \boldsymbol{u}_{c a}(k)$ and $\boldsymbol{L} \boldsymbol{u}_{c a}(k-$ 1) results in

$$
\begin{aligned}
\boldsymbol{u}_{a c t}(k)-\boldsymbol{D} \hat{\boldsymbol{E}} \boldsymbol{F} \boldsymbol{\tau}_{a c t}(k-1) & -\boldsymbol{D} \hat{\boldsymbol{E}} \boldsymbol{G} \boldsymbol{u}_{d}(k) \\
& =\boldsymbol{L} \boldsymbol{u}_{d}(k) \\
& +\boldsymbol{u}_{a c t}(k-1)-\boldsymbol{D} \hat{\boldsymbol{E}} \boldsymbol{F} \boldsymbol{\tau}_{a c t}(k-2) \\
& -\boldsymbol{D} \hat{\boldsymbol{E}} \boldsymbol{G} \boldsymbol{u}_{d}(k-1)-\boldsymbol{L} \boldsymbol{u}_{a c t}(k-1)
\end{aligned}
$$

Rearranging and collecting like terms yields

$$
\begin{aligned}
\boldsymbol{u}_{a c t}(k) & -(\boldsymbol{I}-\boldsymbol{L}) \boldsymbol{u}_{a c t}(k-1) \\
& =(\boldsymbol{L}+\boldsymbol{D} \hat{\boldsymbol{E}} \boldsymbol{G}) \boldsymbol{u}_{d}(k)-\boldsymbol{D} \hat{\boldsymbol{E}} \boldsymbol{G} \boldsymbol{u}_{d}(k-1) \\
& +\boldsymbol{D} \hat{\boldsymbol{E}} \boldsymbol{F}\left(\boldsymbol{\tau}_{a c t}(k-1)-\boldsymbol{\tau}_{a c t}(k-2)\right)
\end{aligned}
$$

Taking the z-transform of Eq. (7.34) gives

$$
\begin{aligned}
{\left[\boldsymbol{I}-z^{-1}(\boldsymbol{I}-\boldsymbol{L})\right] \boldsymbol{U}_{a c t}(z) } & =\left[\boldsymbol{L}+\boldsymbol{D} \hat{\boldsymbol{E}} \boldsymbol{G}-z^{-1} \boldsymbol{D} \hat{\boldsymbol{E}} \boldsymbol{G}\right] \boldsymbol{U}_{d}(z) \\
& +\boldsymbol{D} \hat{\boldsymbol{E}} \boldsymbol{F} z^{-1}\left(1-z^{-1}\right) \boldsymbol{\Gamma}_{a c t}(z)
\end{aligned}
$$


The characteristic equation of the closed loop is [41]

$$
\left|\boldsymbol{I}-z^{-1}(\boldsymbol{I}-\boldsymbol{L})\right|=0
$$

which can be manipulated into

$$
|z \boldsymbol{I}-(\boldsymbol{I}-\boldsymbol{L})|=0
$$

Based on the fundamental theory for discrete-time systems, in order for the system to be stable, the norm of all the characteristic roots should be less than $1[41,43]$. That is the roots of $\left|z_{i}\right|=\left|1-\lambda_{i}\right|<1$.

To prove the stability, let $\boldsymbol{L}=\boldsymbol{D} \hat{\boldsymbol{E}} \boldsymbol{H}(\boldsymbol{D} \hat{\boldsymbol{E}})^{+}$and by making use of $\boldsymbol{H}$ and $\boldsymbol{G}$ matrices such that

$$
\begin{aligned}
\boldsymbol{L} & =\boldsymbol{D} \hat{\boldsymbol{E}}\left[\left(\boldsymbol{I}-\boldsymbol{W}^{-1}\left(\boldsymbol{D} \hat{\boldsymbol{E}} \boldsymbol{W}^{-1}\right)^{+} \boldsymbol{D} \hat{\boldsymbol{E}}\right) \boldsymbol{W}^{-2} \boldsymbol{W}_{1}^{2}\right](\boldsymbol{D} \hat{\boldsymbol{E}})^{+} \\
& =\boldsymbol{D} \hat{\boldsymbol{E}}\left[\left(\boldsymbol{W}^{-1}-\boldsymbol{W}^{-1}\left(\boldsymbol{D} \hat{\boldsymbol{E}} \boldsymbol{W}^{-1}\right)^{+} \boldsymbol{D} \hat{\boldsymbol{E}} \boldsymbol{W}^{-1}\right) \boldsymbol{W}^{-1} \boldsymbol{W}_{1}^{2}\right](\boldsymbol{D} \hat{\boldsymbol{E}})^{+} \\
& =\boldsymbol{D} \hat{\boldsymbol{E}} \boldsymbol{W}^{-1}\left(\boldsymbol{I}-\left(\boldsymbol{D} \hat{\boldsymbol{E}} \boldsymbol{W}^{-1}\right)^{+} \boldsymbol{D} \hat{\boldsymbol{E}} \boldsymbol{W}^{-1}\right) \boldsymbol{W}^{-1} \boldsymbol{W}_{1}^{2}(\boldsymbol{D} \hat{\boldsymbol{E}})^{+}
\end{aligned}
$$

The singular value decomposition (SVD) of $\boldsymbol{D} \hat{\boldsymbol{E}}$ and $\boldsymbol{D} \hat{\boldsymbol{E}} \boldsymbol{W}^{-1}$ is as follows

$$
\begin{aligned}
\boldsymbol{D} \hat{\boldsymbol{E}} & =\boldsymbol{U}_{1} \boldsymbol{\Sigma}_{1} \boldsymbol{V}_{1}^{T} \\
& =\boldsymbol{U}_{1}\left[\begin{array}{ll}
\boldsymbol{\Sigma}_{1 r} & 0
\end{array}\right]\left[\begin{array}{c}
\boldsymbol{V}_{1 r}^{T} \\
\boldsymbol{V}_{1 o}^{T}
\end{array}\right] \\
& =\boldsymbol{U}_{1} \boldsymbol{\Sigma}_{1 r} \boldsymbol{V}_{1 r}^{T} \\
\boldsymbol{D} \hat{\boldsymbol{E}} \boldsymbol{W}^{-1} & =\boldsymbol{U}_{2} \boldsymbol{\Sigma}_{2} \boldsymbol{V}_{2}^{T} \\
& =\boldsymbol{U}_{2}\left[\begin{array}{ll}
\boldsymbol{\Sigma}_{2 r} & 0
\end{array}\right]\left[\begin{array}{c}
\boldsymbol{V}_{2 r}^{T} \\
\boldsymbol{V}_{2 o}^{T}
\end{array}\right] \\
& =\boldsymbol{U}_{2} \boldsymbol{\Sigma}_{2 r} \boldsymbol{V}_{2 r}^{T}
\end{aligned}
$$

Using SDV, the term $\boldsymbol{I}-\left(\boldsymbol{D} \hat{\boldsymbol{E}} \boldsymbol{W}^{-1}\right)^{+} \boldsymbol{D} \hat{\boldsymbol{E}} \boldsymbol{W}^{-1}$ in Eq. (7.38) can be expressed as

$$
\begin{aligned}
\boldsymbol{I}-\boldsymbol{V}_{2 r} \boldsymbol{\Sigma}_{2 r}^{-1} \boldsymbol{U}_{2}^{T} \boldsymbol{U}_{2} \boldsymbol{\Sigma}_{2 r} \boldsymbol{V}_{2 r}^{T} & =\boldsymbol{I}-\boldsymbol{V}_{2 r} \boldsymbol{V}_{2 r}^{T} \\
& =\boldsymbol{V}_{2 o} \boldsymbol{V}_{2 o}^{T}
\end{aligned}
$$

since $\boldsymbol{V}_{2} \boldsymbol{V}_{2}^{T}=\boldsymbol{V}_{2 o} \boldsymbol{V}_{2 o}^{T}+\boldsymbol{V}_{2 r} \boldsymbol{V}_{2 r}^{T}=\boldsymbol{I}[40]$ and SDV of any pseudo-inverse, $(\boldsymbol{A})^{+}$, is $\boldsymbol{V} \boldsymbol{\Sigma}^{-1} \boldsymbol{U}^{T}[78]$ 
Substituting Eq. (7.41) into Eq. (7.38), using SDV furthermore, and making use of $\lambda(\boldsymbol{A} \boldsymbol{B})=\lambda(\boldsymbol{B} \boldsymbol{A})$ property gives $[40,41]$

$$
\begin{aligned}
\boldsymbol{L} & =\boldsymbol{U}_{1} \boldsymbol{\Sigma}_{1 r} \boldsymbol{V}_{1 r}^{T} \boldsymbol{W}^{-1} \boldsymbol{V}_{2 o} \boldsymbol{V}_{2 o}^{T} \boldsymbol{W}^{-1} \boldsymbol{W}_{1}^{2} \boldsymbol{V}_{1 r} \boldsymbol{\Sigma}_{r 1}^{-1} \boldsymbol{U}_{1}^{T} \\
\lambda(\boldsymbol{L}) & =\lambda\left(\boldsymbol{V}_{2 o}^{T} \boldsymbol{W}^{-1} \boldsymbol{W}_{1}^{2} \boldsymbol{V}_{1 r} \boldsymbol{\Sigma}_{r 1}^{-1} \boldsymbol{U}_{1}^{T} \boldsymbol{U}_{1} \boldsymbol{\Sigma}_{1 r} \boldsymbol{V}_{1 r}^{T} \boldsymbol{W}^{-1} \boldsymbol{V}_{2 o}\right) \\
& =\lambda\left(\boldsymbol{V}_{2 o}^{T} \boldsymbol{W}^{-1} \boldsymbol{W}_{1}^{2} \boldsymbol{W}^{-1} \boldsymbol{V}_{2 o}\right) \\
& =\lambda\left(\boldsymbol{V}_{2 o}^{T} \boldsymbol{W}^{-1} \boldsymbol{W}_{1}^{T} \boldsymbol{W}_{1} \boldsymbol{W}^{-1} \boldsymbol{V}_{2 o}\right)
\end{aligned}
$$

Using the definition of maximum singular value, denoted by $\sigma_{\max }^{2}(\boldsymbol{X})=\lambda\left(\boldsymbol{X}^{T} \boldsymbol{X}\right)$, one gets

$$
\lambda\left(\boldsymbol{V}_{2 o}^{T} \boldsymbol{W}^{-1} \boldsymbol{W}_{1}^{2} \boldsymbol{W}^{-1} \boldsymbol{V}_{2 o}\right)=\sigma_{\max }^{2}\left(\boldsymbol{W}_{1} \boldsymbol{W}^{-1} \boldsymbol{V}_{2 o}\right)>0
$$

This states that the nonzero eigenvalues of $\boldsymbol{L}$ are real and positive [41].

By using $\sigma_{\max }^{2}(\boldsymbol{X})=\|\boldsymbol{X}\|^{2}$, Eq. (7.43) can be rewritten as [41]

$$
\begin{aligned}
\sigma_{\max }^{2}\left(\boldsymbol{W}_{1} \boldsymbol{W}^{-1} \boldsymbol{V}_{2 o}\right) & =\left\|\boldsymbol{W}_{1} \boldsymbol{W}^{-1} \boldsymbol{V}_{2 o}\right\|^{2} \\
& \leqslant\left\|\boldsymbol{W}_{1} \boldsymbol{W}^{-1}\right\|^{2}\left\|\boldsymbol{V}_{2 o}\right\|^{2} \\
& =\left\|\boldsymbol{W}_{1} \boldsymbol{W}^{-1}\right\|^{2}
\end{aligned}
$$

where $\lambda\left(\boldsymbol{V}_{2 o}^{T} \boldsymbol{V}_{2 o}\right)=1$.

The largest singular value can be expressed as

$$
\lambda_{\max }(\boldsymbol{L})=\sup _{x \neq 0} \frac{\boldsymbol{x}^{T} \boldsymbol{W}^{-1} \boldsymbol{W}_{1}^{2} \boldsymbol{W}^{-1} \boldsymbol{x}}{\boldsymbol{x}^{T} \boldsymbol{x}}
$$

By defining $\boldsymbol{x}=\boldsymbol{W} \boldsymbol{y}$ and $\boldsymbol{y}=\boldsymbol{W}^{-1} \boldsymbol{x}$, one gets [41]

$$
\begin{aligned}
\lambda_{\max }(\boldsymbol{L}) & =\sup _{y \neq 0} \frac{\boldsymbol{y}^{T} \boldsymbol{W}_{1}^{2} \boldsymbol{y}}{\boldsymbol{y}^{T} \boldsymbol{W}^{T} \boldsymbol{W} \boldsymbol{y}} \\
& =\sup _{y \neq 0} \frac{\boldsymbol{y}^{T} \boldsymbol{W}_{1}^{2} \boldsymbol{y}}{\boldsymbol{y}^{T}\left[\boldsymbol{W}_{0}^{2}+\boldsymbol{W}_{1}^{2}+\boldsymbol{W}_{2}^{2}\right] \boldsymbol{y}} \\
& \leqslant \sup _{y \neq 0} \frac{\boldsymbol{y}^{T} \boldsymbol{W}_{1}^{2} \boldsymbol{y}}{\boldsymbol{y}^{T} \boldsymbol{W}_{1}^{2} \boldsymbol{y}}=1
\end{aligned}
$$

Since the eigenvalues are less than one and greater than zero, the system is asymptotically stable [41]. 


\subsection{Modified Closed Loop Control Allocation}

The proposed modified closed-loop control allocation (MCLCA) designed by Qiao et al. [43] is a modified version of the CLCA, which was implemented by $\mathrm{Hu}$ et al. [41]. The advantage of the MCLCA is that the modification reduces the allocation error under actuator uncertainties and increases the convergence rate of the CA [43]. The MCLCA has never been tested for systems with faults.

The MCLCA system, graphically shown in Fig. 7.2, can be described as

$$
\begin{aligned}
{\left[\boldsymbol{I}-z^{-1}(\boldsymbol{I}-\boldsymbol{N})\right] \boldsymbol{U}_{a c t}(z) } & =\boldsymbol{N} \boldsymbol{U}_{d}(z) \\
& +\boldsymbol{D} \hat{\boldsymbol{E}} \boldsymbol{F} z^{-1}\left(1-z^{-1}\right) \boldsymbol{\Gamma}_{a c t}(z)
\end{aligned}
$$

where the eigenvalues of $\boldsymbol{N}$ are in the domain $(0,1)$, guaranteeing that the system is asymptotically stable.

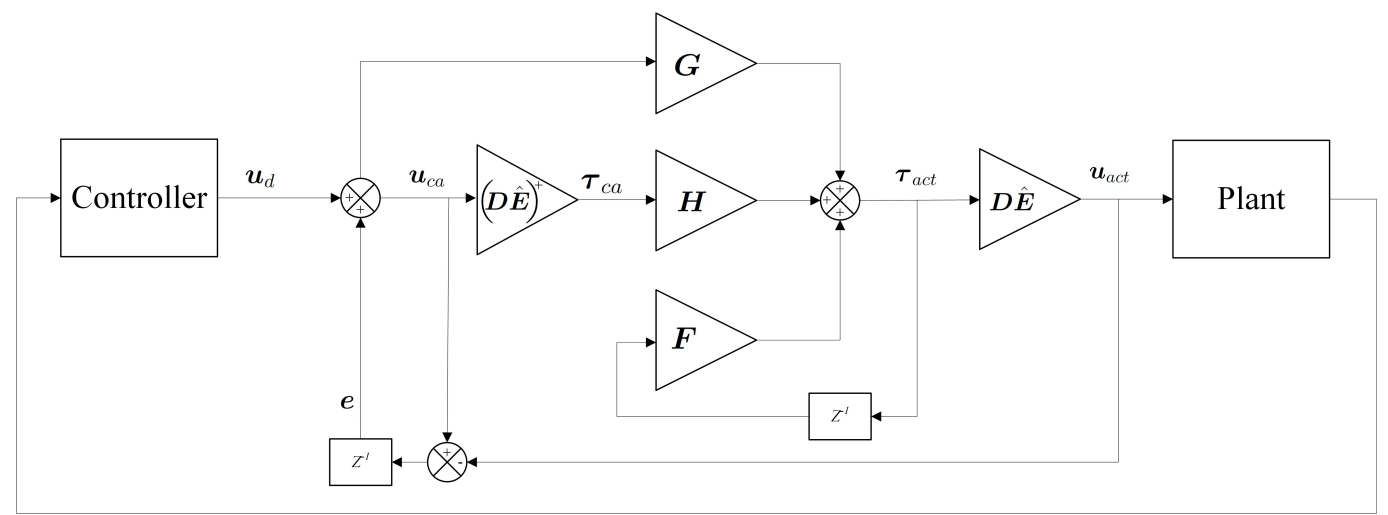

Figure 7.2: Modified Closed Loop Control Allocation Diagram

Proof: The MCLCA based on the work in [43], is given by

$$
\boldsymbol{\tau}_{a c t}(k)=\boldsymbol{H} \boldsymbol{\tau}_{c a}+\boldsymbol{F} \boldsymbol{\tau}_{a c t}(k-1)+\boldsymbol{G} \boldsymbol{u}_{c a}(k)
$$

Compared with the work of $\mathrm{Hu}$ et al. [41], the allocation error is also fed into the matrix $\boldsymbol{G}$. In most cases, $\boldsymbol{G}$ is a weighting part of Eq. (7.48) compared to $\boldsymbol{H}$ and $\boldsymbol{F}$. Having the allocation error shared among the three terms contributes to a better performance [43].

Substituting Eqs. (7.25) and (7.26) into Eq. (7.48) yields

$$
\boldsymbol{u}_{a c t}(k)=\boldsymbol{D} \hat{\boldsymbol{E}} \boldsymbol{H}(\boldsymbol{D} \hat{\boldsymbol{E}})^{+} \boldsymbol{u}_{c a}(k)+\boldsymbol{D} \hat{\boldsymbol{E}} \boldsymbol{F} \boldsymbol{\tau}_{a c t}(k-1)+\boldsymbol{D} \hat{\boldsymbol{E}} \boldsymbol{G} \boldsymbol{u}_{c a}(k)
$$


Equation (7.49) can be rearranged as follows

$$
\left(\boldsymbol{D} \hat{\boldsymbol{E}} \boldsymbol{H}(\boldsymbol{D} \hat{\boldsymbol{E}})^{+}+\boldsymbol{D} \hat{\boldsymbol{E}} \boldsymbol{G}\right) \boldsymbol{u}_{c a}(k)=\boldsymbol{u}_{a c t}(k)-\boldsymbol{D} \hat{\boldsymbol{E}} \boldsymbol{F} \boldsymbol{\tau}_{a c t}(k-1)
$$

Letting $\boldsymbol{N}=\boldsymbol{D} \hat{\boldsymbol{E}} \boldsymbol{H}(\boldsymbol{D} \hat{\boldsymbol{E}})^{+}+\boldsymbol{D} \hat{\boldsymbol{E}} \boldsymbol{G}$ and multiplying Eq. (7.31) by $\boldsymbol{N}$ gives

$$
\boldsymbol{N} \boldsymbol{u}_{c a}(k)=\boldsymbol{N} \boldsymbol{u}_{d}(k)+\boldsymbol{N} \boldsymbol{u}_{c a}(k-1)-\boldsymbol{N} \boldsymbol{u}_{a c t}(k-1)
$$

Substituting Eq. (7.50) into (7.51) for $\boldsymbol{u}_{c a}(k)$ and $\boldsymbol{u}_{c a}(k-1)$ yields

$$
\begin{aligned}
\boldsymbol{u}_{a c t}(k)-\boldsymbol{D} \hat{\boldsymbol{E}} \boldsymbol{F} \boldsymbol{\tau}_{a c t}(k-1) & =\boldsymbol{N} \boldsymbol{u}_{d}(k)+\boldsymbol{u}_{a c t}(k-1) \\
& -\boldsymbol{D} \hat{\boldsymbol{E}} \boldsymbol{F} \boldsymbol{\tau}_{\text {act }}(k-2)-\boldsymbol{N} \boldsymbol{u}_{\text {act }}(k-1)
\end{aligned}
$$

which can be rearranged as follows

$$
\begin{aligned}
\boldsymbol{u}_{a c t}(k)-(\boldsymbol{I}-\boldsymbol{N}) \boldsymbol{u}_{a c t}(k-1) & =\boldsymbol{N} \boldsymbol{u}_{d}(k) \\
& +\boldsymbol{D} \hat{\boldsymbol{E}} \boldsymbol{F}\left(\boldsymbol{\tau}_{\text {act }}(k-1)-\boldsymbol{\tau}_{\text {act }}(k-2)\right)
\end{aligned}
$$

Taking the z-transform of Eq. (7.53) gives

$$
\begin{aligned}
{\left[\boldsymbol{I}-z^{-1}(\boldsymbol{I}-\boldsymbol{N})\right] \boldsymbol{U}_{a c t}(z) } & =\boldsymbol{N} \boldsymbol{U}_{d}(z) \\
& +\boldsymbol{D} \hat{\boldsymbol{E}} \boldsymbol{F} z^{-1}\left(1-z^{-1}\right) \boldsymbol{\Gamma}_{a c t}(z)
\end{aligned}
$$

The characteristic equation of Eq. (7.54) is

$$
|z \boldsymbol{I}-(\boldsymbol{I}-\boldsymbol{N})|=0
$$

\section{Stability Proof}

For stability, the sufficient condition is [43]

$$
|z|=\left|1-\lambda_{i}\right|<1
$$

where $\lambda_{i}$ are the eigenvalues of the matrix $\boldsymbol{N}$. 
First, by making use of the definition of $\boldsymbol{N}, \boldsymbol{G}$, and $\boldsymbol{L}$, the following can be obtained [43]

$$
\begin{aligned}
\boldsymbol{N} & =\boldsymbol{D} \hat{\boldsymbol{E}} \boldsymbol{H}(\boldsymbol{D} \hat{\boldsymbol{E}})^{+}+\boldsymbol{D} \hat{\boldsymbol{E}} \boldsymbol{G} \\
& =\boldsymbol{D} \hat{\boldsymbol{E}} \boldsymbol{H}(\boldsymbol{D} \hat{\boldsymbol{E}})^{+}+\boldsymbol{D} \hat{\boldsymbol{E}} \boldsymbol{W}^{-1}\left(\boldsymbol{D} \hat{\boldsymbol{E}} \boldsymbol{W}^{-1}\right)^{+} \\
& =\boldsymbol{D} \hat{\boldsymbol{E}} \boldsymbol{H}(\boldsymbol{D} \hat{\boldsymbol{E}})^{+}+\boldsymbol{I} \\
& =\boldsymbol{L}+\boldsymbol{I}
\end{aligned}
$$

From before it was shown that the eigenvalues of $\boldsymbol{L}$ satisfy

$$
0<\lambda(\boldsymbol{L})<1
$$

Using $\boldsymbol{N}=\boldsymbol{L}+\boldsymbol{I}$ and manipulating it into $\boldsymbol{I}-\boldsymbol{N}=-\boldsymbol{L}$ the above can be rewritten as [43]

$$
-1<\lambda(\boldsymbol{I}-\boldsymbol{N})<0
$$

which simplifies to

$$
-1<1-\lambda(\boldsymbol{N})_{i}<0
$$

The norm of the characteristic root is less than one, therefore the system is asymptotically stable based on the fundamental theory for discrete-time systems. 


\section{Chapter 8}

\section{Results}

This chapter highlights the results of the thesis. All simulations are performed using MATLAB/Simulink. Four control laws are studied in numerical simulations. The first three control are used as a benchmark and they are NTSMC, MNTSMC, OSMC. The fourth one is SAC, which is the main contribution of this thesis. The performance of these control laws in investigated under nominal conditions, in a scenario where the plant experiences large perturbations, and faults. Three types of faults are considered: total loss of effectiveness, locked in place actuators, and partial loss of actuation. The second part of this chapter focuses on the results for CA. More specifically, the results of three CA algorithms are studied. These algorithms are: weighted pseudo inverse (WPI), and two closed-loop algorithms based on quadratic programming. Two different scenarios are implemented in the numerical simulator. For the second scenario, the fault are more severe compared to the first scenario.

Section 8.1: Introduction presents the parameters set in the simulation. This includes the orbital elements used in simulation, the control law parameters for the various control laws, the control allocation matrix, and other necessary parameters required for CLCA. The desired position of the chaser in detailed in Sec. 8.2: Desired Formation. The results for the nominal case are highlighted in Sec. 8.3: Nominal Case, followed by the results for the extra perturbation scenario in Sec. 8.4: Extra Perturbations. Section 8.5: Fault Case presents the results for the three fault scenarios and explains the concept of resetting the ideal model for SAC. Lastly, the results of CA are given in Sec. 8.6: Control Allocation. 


\subsection{Introduction}

This section highlights the key parameters that are used in the numerical simulator. Table 8.1 provides the orbital elements as well as the mass of the chaser and the target spacecraft used in the simulation. The control gains for NTSMC and MNTSMC are provided in Table 8.2, OSMC control parameters are given in Table 8.3, and SAC parameters are presented in Table 8.4. The control laws were turned by trial and error such that all four control laws will all have the same performance under nominal condition.

Table 8.1: Orbital and System Parameters

\begin{tabular}{cc}
\hline \hline Parameter & Value \\
\hline$m_{c, t}$ & $10 \mathrm{~kg}$ \\
$r_{p}$ & $6878 \mathrm{~km}$ \\
$e$ & 0.2 \\
$\Delta e$ & $2.9 \mathrm{e}-6$ \\
$\Omega, \omega, i, M$ & $0^{\circ}$ \\
\hline \hline
\end{tabular}

Table 8.2: Terminal Sliding Mode Control Parameters

\begin{tabular}{c||cc}
\hline \hline & $\mathrm{NTSMC}$ & $\mathrm{MNTSMC}$ \\
\hline $\boldsymbol{K}$ & $30 \boldsymbol{I}_{3}$ & $15 \boldsymbol{I}_{3}$ \\
$\{\mathrm{p}, \mathrm{q}\}$ & $\{15,13\}$ & $\{15,13\}$ \\
$\boldsymbol{\eta}$ & $0.96 \boldsymbol{I}_{3}$ & $0.1 \boldsymbol{I}_{3}$ \\
$\gamma$ & 0.45 & $\mathrm{NA}$ \\
$\boldsymbol{G}$ & $0.15 \boldsymbol{I}_{3}$ & $\mathrm{NA}$ \\
\hline \hline
\end{tabular}

Table 8.3: OSMC Parameters

\begin{tabular}{cc}
\hline \hline $\boldsymbol{Q}$ & $8 \mathrm{e} 17 \boldsymbol{I}_{6}$ \\
$\boldsymbol{R}$ & $1.3 \mathrm{e} 20 \boldsymbol{I}_{3}$ \\
$\boldsymbol{\lambda}$ & $5 \mathrm{e}-4 \boldsymbol{I}_{3}$ \\
$\gamma$ & 0.45 \\
\hline \hline
\end{tabular}


Table 8.4: SAC Parameters

\begin{tabular}{cccc}
\hline \hline$\Gamma_{I e}$ & $5 \mathrm{e} 2 \boldsymbol{I}_{3}$ & $\Gamma_{P e}$ & $4 \mathrm{e} 3 \boldsymbol{I}_{3}$ \\
$\Gamma_{I u}$ & $1 \mathrm{e}-2 \boldsymbol{I}_{3}$ & $\Gamma_{P u}$ & $1 \mathrm{e} 4 \boldsymbol{I}_{3}$ \\
$\Gamma_{I x}$ & $1 \mathrm{e}-2 \boldsymbol{I}_{6}$ & $\Gamma_{P x}$ & $1 \mathrm{e} 4 \boldsymbol{I}_{6}$ \\
$\omega_{n}$ & 0.13 & $\zeta$ & 0.9 \\
$\alpha$ & 1 & & \\
\hline \hline
\end{tabular}

For control allocation, the actuator distribution matrix, $\boldsymbol{D}$, is the summation of the nominal values of the distribution plus the uncertainty of the distribution [41]. Mathematically, this is expressed as

$$
D=D_{0}+\Delta D
$$

The nominal matrix is expressed as [41]

$$
\boldsymbol{D}_{0}=\left[\begin{array}{cccc}
1 & 0 & 0 & \cos \alpha_{4} \cos \beta_{4} \\
0 & 1 & 0 & \cos \alpha_{4} \sin \beta_{4} \\
0 & 0 & 1 & \sin \alpha_{4}
\end{array}\right]
$$

and the uncertainty matrix is given by [41]

$\Delta D=$

$\left[\begin{array}{cccc}0 & \Delta \alpha_{2} \cos \Delta \beta_{2} & \Delta \alpha_{3} \cos \Delta \beta_{3} & -\Delta \alpha_{4} \sin \alpha_{4} \cos \beta_{4}-\Delta \beta_{4} \cos \alpha_{4} \sin \beta_{4} \\ \Delta \alpha_{1} \cos \Delta \beta_{1} & 0 & \Delta \alpha_{3} \sin \Delta \beta_{3} & -\Delta \alpha_{4} \sin \alpha_{4} \sin \beta_{4}+\Delta \beta_{4} \cos \alpha_{4} \cos \beta_{4} \\ \Delta \alpha_{1} \sin \Delta \beta_{1} & \Delta \alpha_{2} \sin \Delta \beta_{2} & 0 & \Delta \alpha_{4} \cos \alpha_{4}\end{array}\right]$

The angles $\alpha$ and $\beta$ as well as the parameters for the CLCA algorithm are provided in Table 8.5.

Table 8.5: CLCA Parameters

\begin{tabular}{cc}
\hline \hline $\boldsymbol{W}_{1}$ & $10 \boldsymbol{I}_{4}$ \\
$\boldsymbol{W}_{2}$ & $15 \boldsymbol{I}_{4}$ \\
$\alpha_{4}$ & $35.26^{\circ}$ \\
$\beta_{4}$ & $45^{\circ}$ \\
$\boldsymbol{\alpha}$ & {$\left[15^{\circ} ; 10^{\circ} ; 15^{\circ} ; 10^{\circ}\right]$} \\
$\boldsymbol{\beta}$ & {$\left[15^{\circ} ; 10^{\circ} ; 15^{\circ} ; 10^{\circ}\right]$} \\
$\delta_{e}$ & $1 \mathrm{e}-5$ \\
\hline \hline
\end{tabular}




\subsection{Desired Formation}

For this thesis, the desired formation is projected circular orbit (PCO). The equation for PCO is given by [19]

$$
\boldsymbol{p}_{d}=\left[\begin{array}{c}
x_{d} \\
y_{d} \\
z_{d}
\end{array}\right]=\frac{r_{p c o}}{2}\left[\begin{array}{c}
\sin (n t) \\
2 \cos (n t) \\
2 \sin (n t)
\end{array}\right]
$$

where $r_{p c o}$ denotes the size of the PCO, chosen to be $1 \mathrm{~km}$, and $n$ is the mean orbital motion of the target spacecraft and $t$ is the time. PCO formation is shown in Fig. 8.1

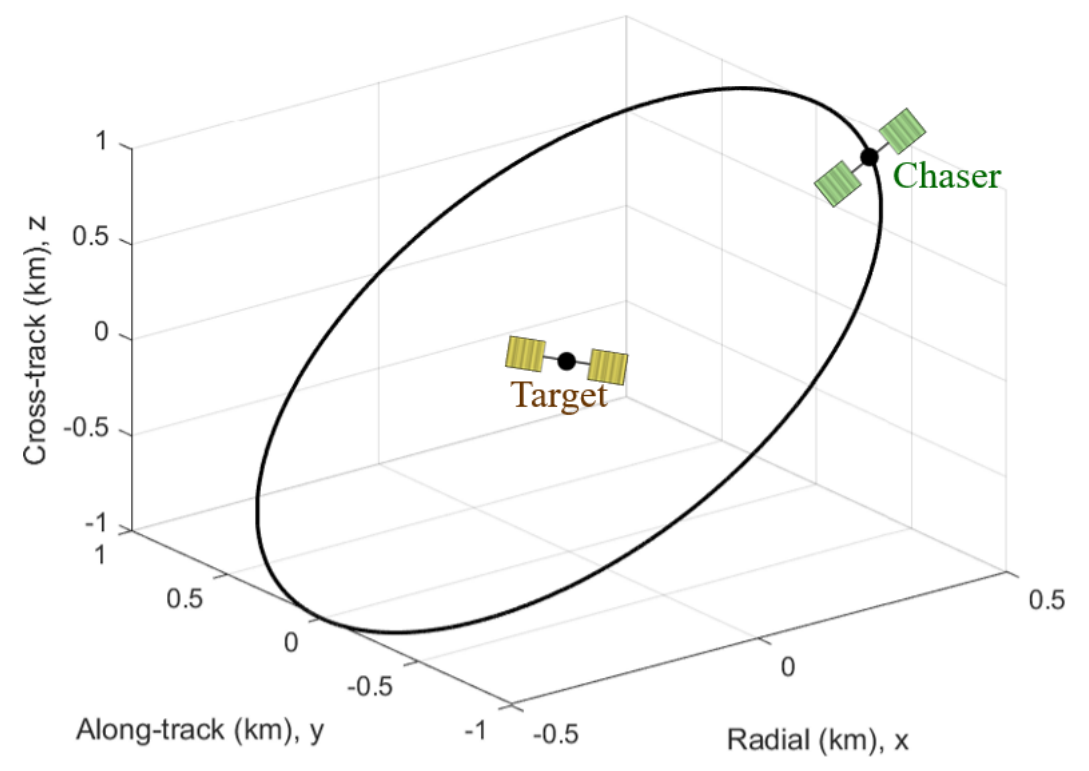

Figure 8.1: PCO Formation 


\subsection{Nominal Case}

This section presents the results for the nominal scenario. Figure 8.2 shows the overall relative position error for the four control laws for a duration of one orbit. Figure 8.3 shows the position error for the first 500 seconds. As seen here, SAC and OSMC have the fastest convergence rate, with OSMC experiencing a higher overshoot than SAC. Figure 8.4 shows the position error during steady state. SAC and OSMC have the lowest position error, with MNTSMC having the worse position error.
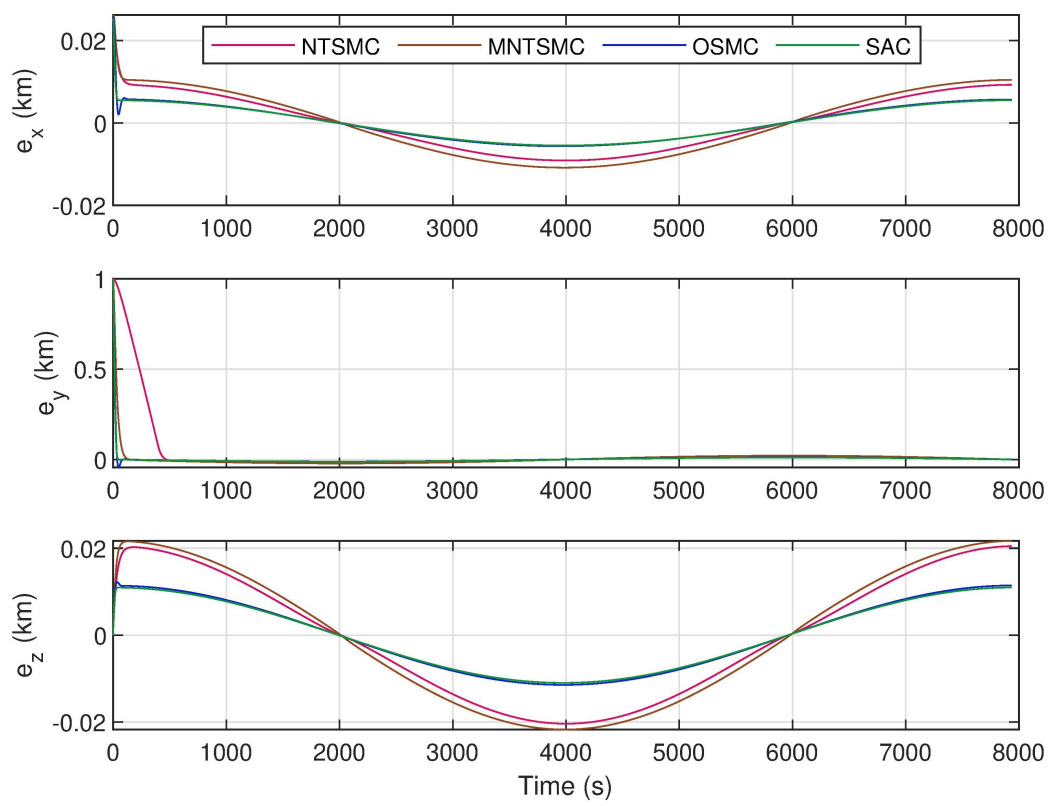

Figure 8.2: Position Error 

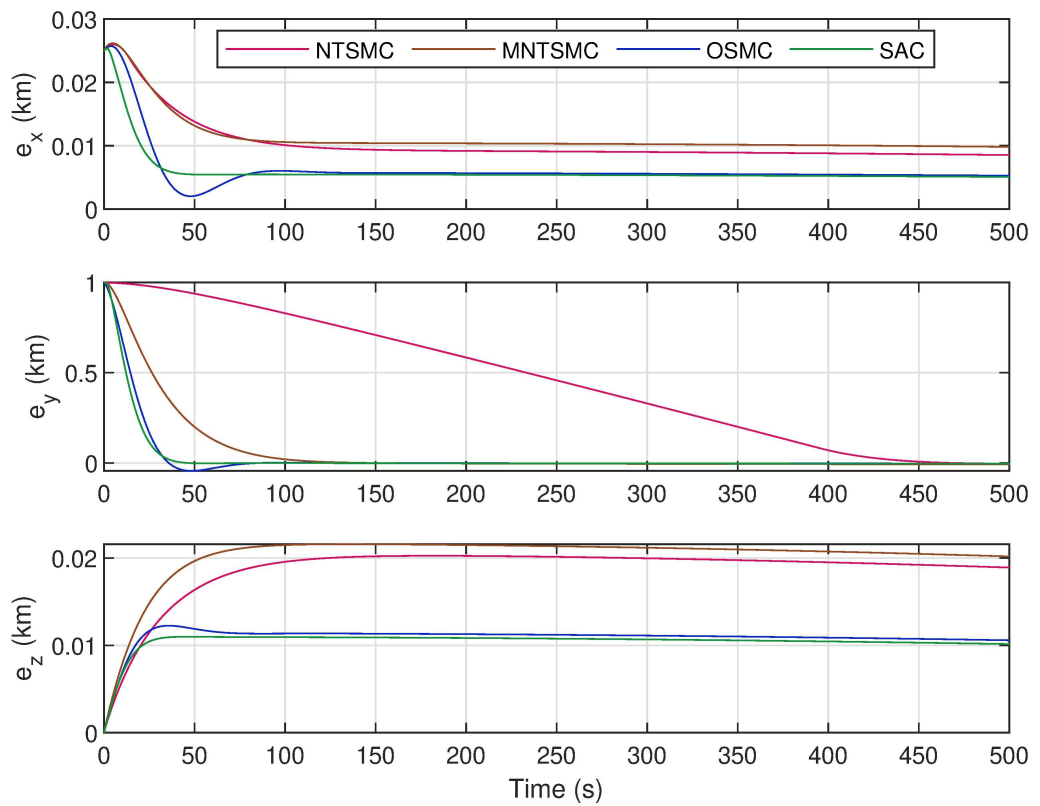

Figure 8.3: Position Error Transient Response
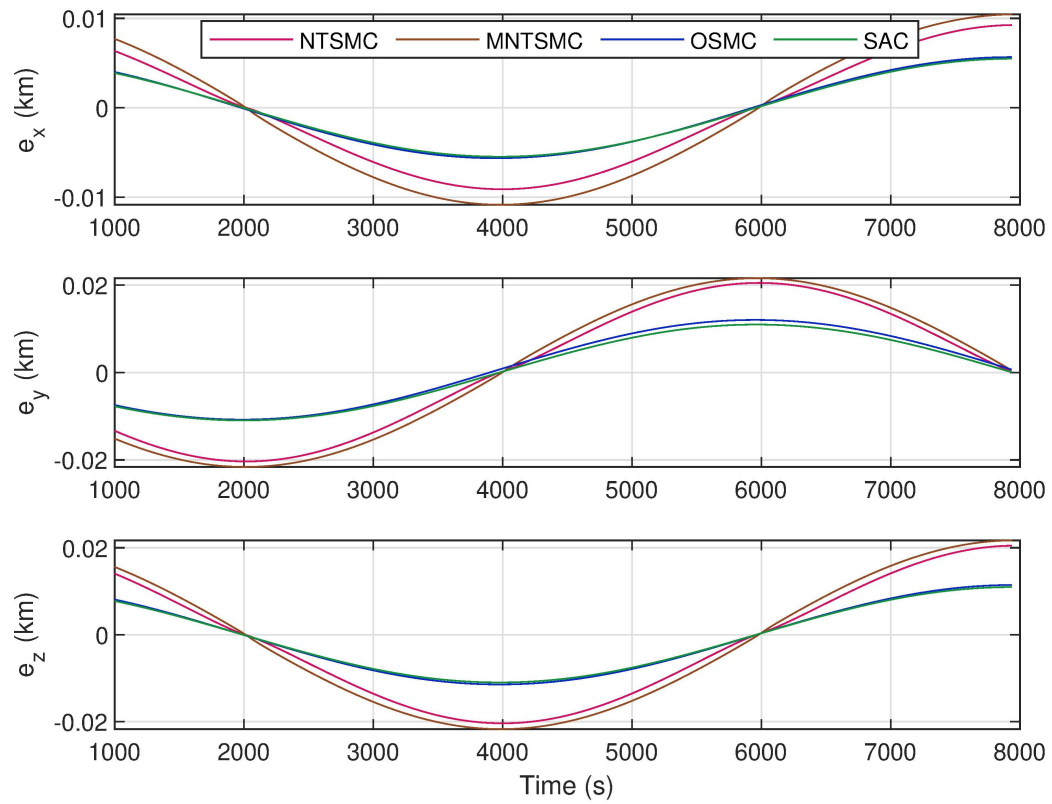

Figure 8.4: Steady State Position Error

Figure 8.5 shows the overall control force of the system. Due to the performance of the system during the transient phase, this figure does not clearly show the behaviour of the control input. Figure 8.6 presents the control force for the 
first 0.25 seconds, showing the transient response of the system. As observed here, NTSMC and MNTSMC require the largest control force and SAC requires the smallest amount of force in the $\mathrm{y}$-axis, where the position error is the largest. Compared to the other control laws, MNTSMC requires significantly more control force in the other two axes as well. The disjointed nature of this figure is due to the step size of 0.1 second, set in the simulation.

The steady state control force is shown in Fig. 8.7. All control laws require the same control force in steady state; however, NTSMC and MNTSMC experience some spikes. This is due to the change of sign for $\boldsymbol{e}$ and $\dot{\boldsymbol{e}}$ at the time of the spike. This causes a sudden change in the value of the control force. More specifically, this change in sign is caused by the sinusoidal nature of the position and velocity of the chaser spacecraft. During the spike time, the corresponding position and velocity vector component cross zero.
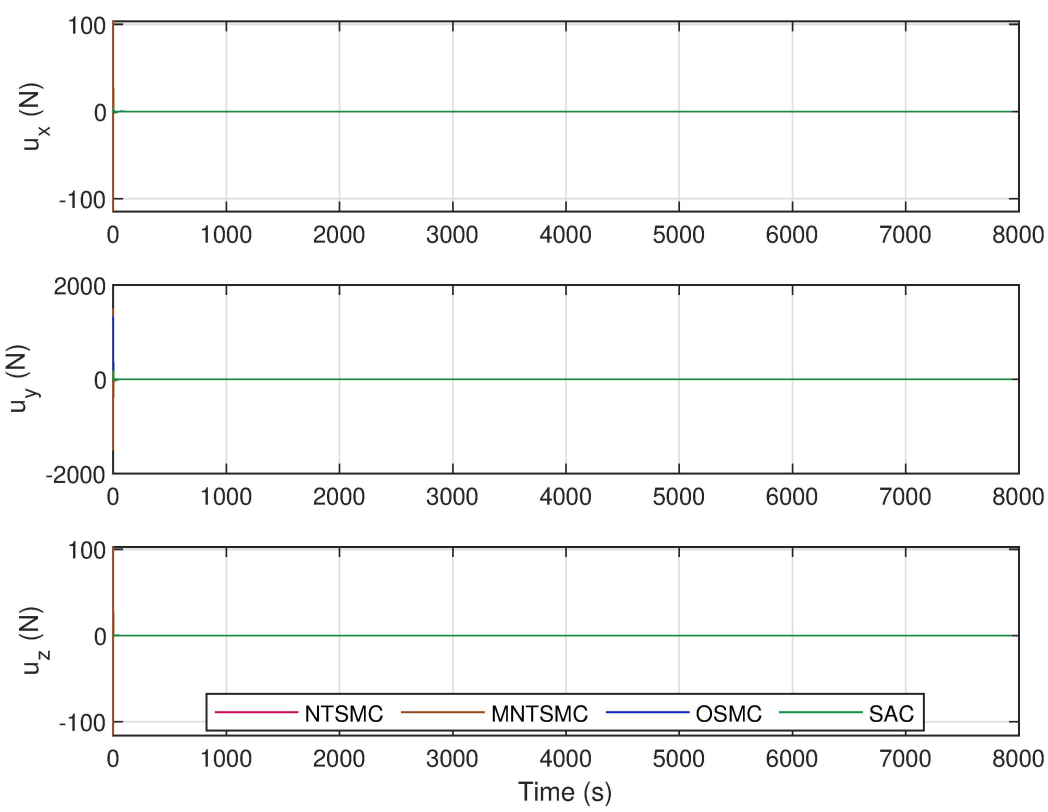

Figure 8.5: Control Force 

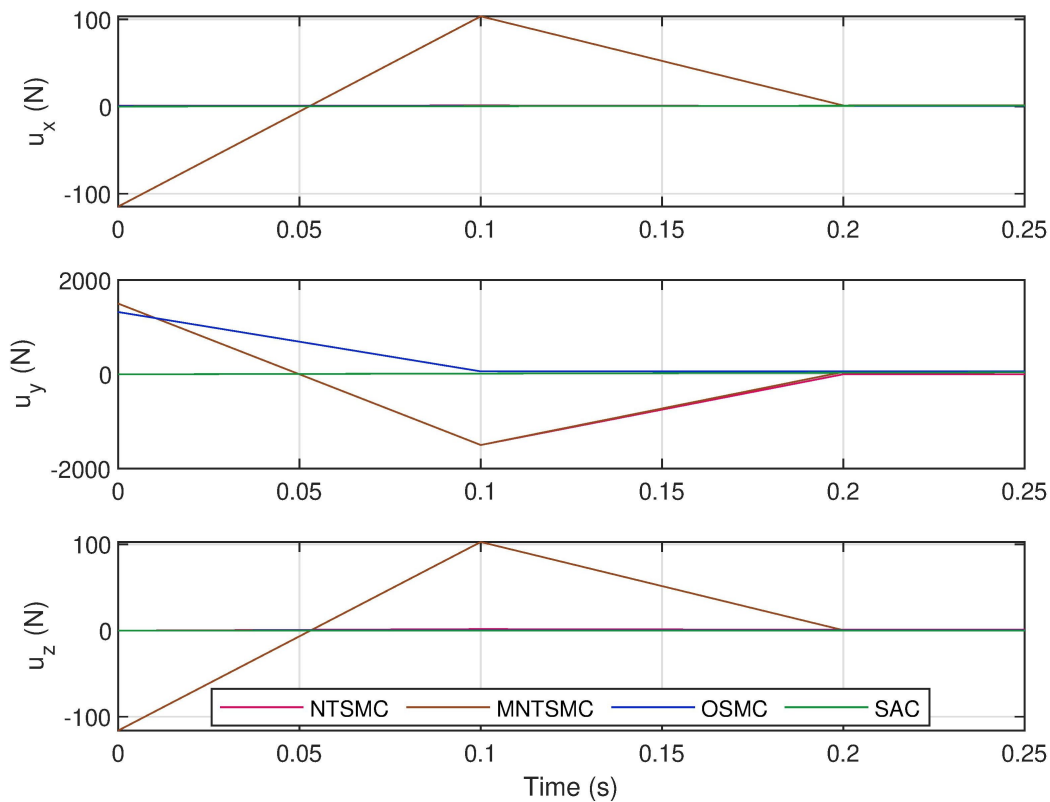

Figure 8.6: Control Force Transient Response
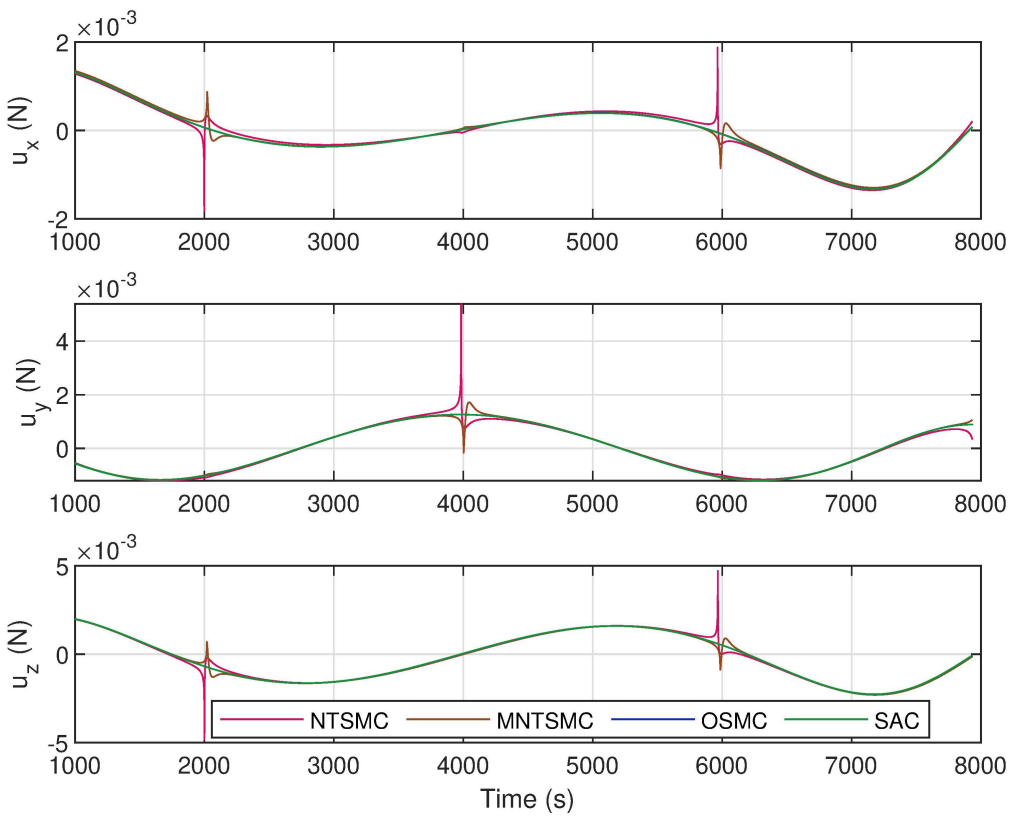

Figure 8.7: Steady State Control Force 


\subsection{Extra Perturbations}

In order to study the importance of having an accurate plant for the model based control laws, the chaser is set to experience large perturbations in the simulation. These perturbations are mathematically modelled as, in $\mathrm{N}$

$$
\left[\begin{array}{c}
F_{d x} \\
F_{d y} \\
F_{d z}
\end{array}\right]=12\left[\begin{array}{c}
1-1.5 \sin (n t) \\
0.5 \sin (2 n t) \\
\sin (n t)
\end{array}\right]
$$

Figure 8.8 shows the control error of the system. As observed, all sliding mode control laws experience a large error while SAC is not effected by these large perturbations. This shows the superiority of SAC, which is not a model based control law, over the other three control laws, which require in one way or another an accurate plant dynamical model.
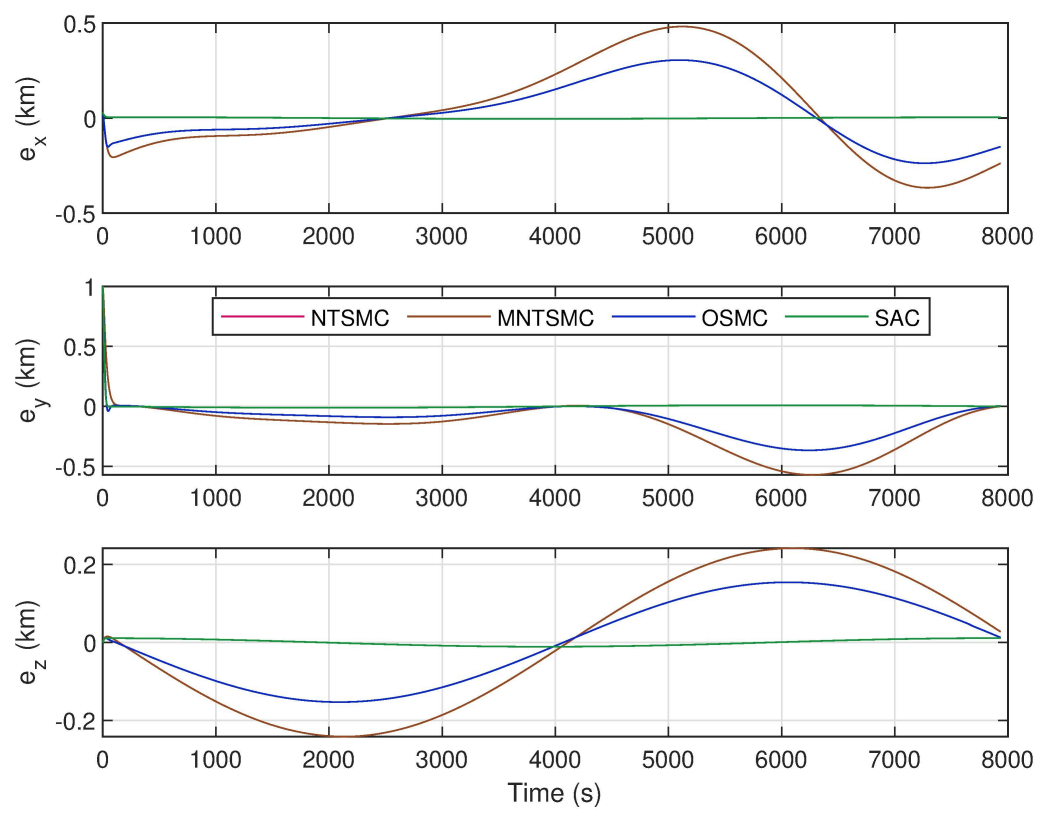

Figure 8.8: Position Error

Figures 8.9 and 8.10 show the control force when the system experiences large perturbations. As seen in Fig. 8.10, all control laws require almost the same amount of force. 

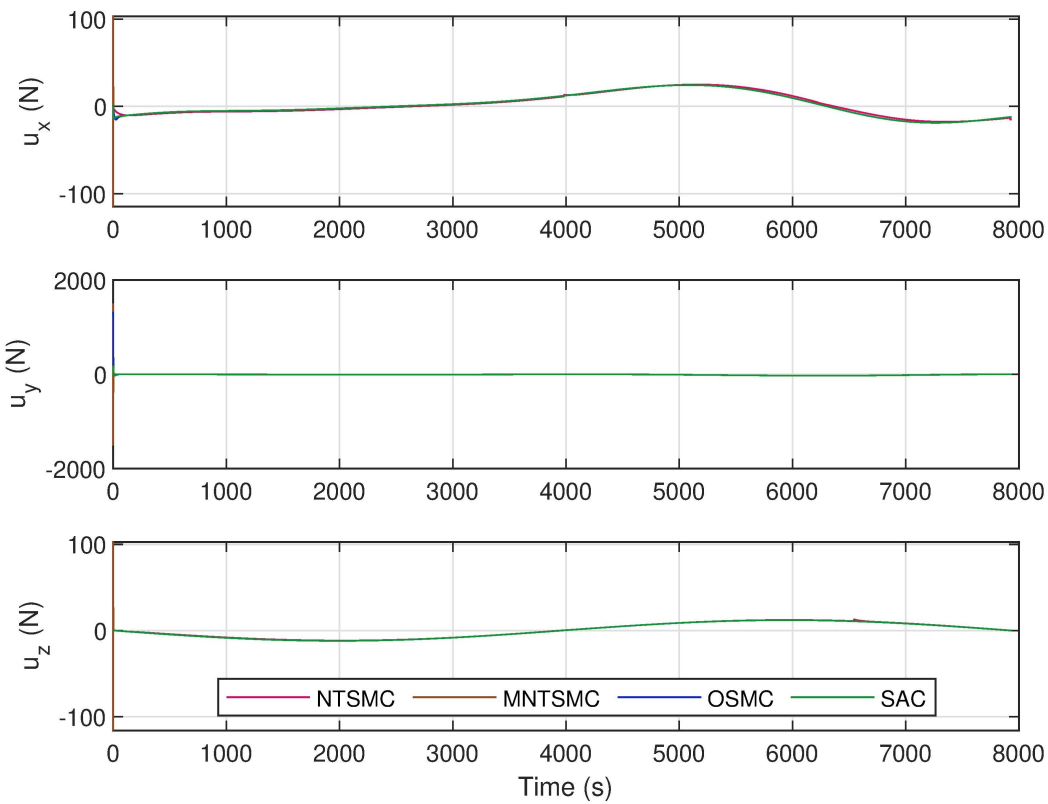

Figure 8.9: Control Force
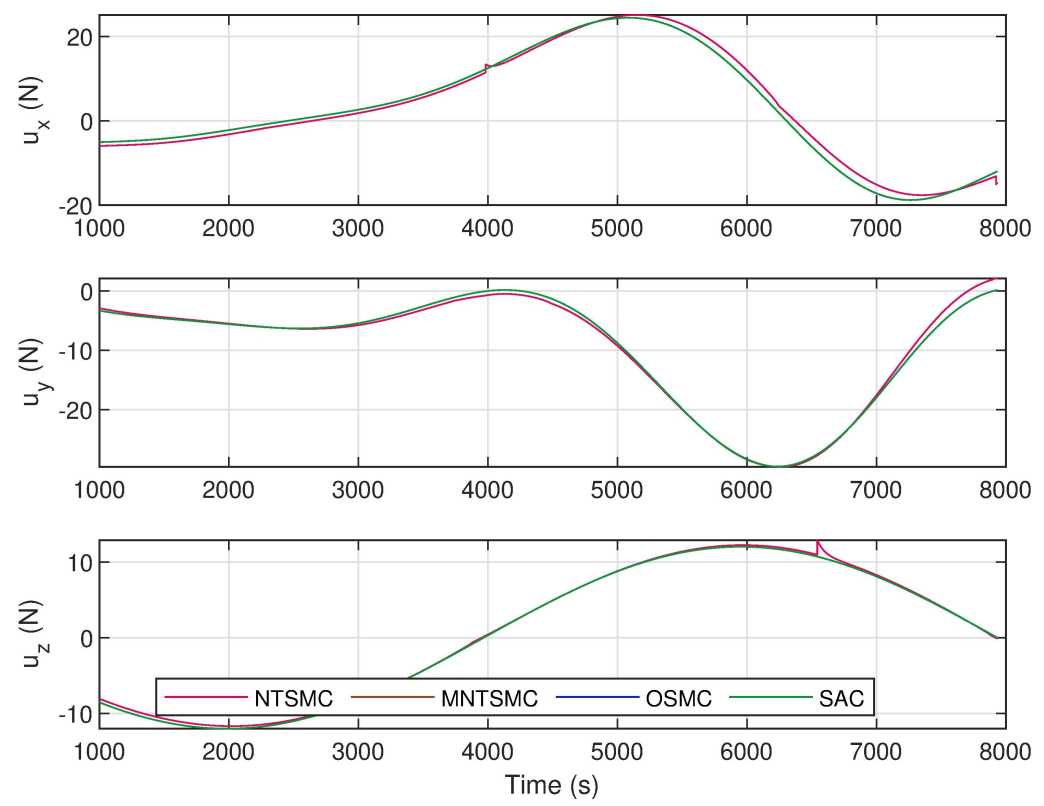

Figure 8.10: Steady State Control Force 


\subsection{Fault Case}

In addition to the nominal and extra perturbation cases, the control laws are tested for three types of fault: total loss of the three actuators, having all three actuators stuck at a certain value, and loss of effectiveness. These results are presented in Secs. 8.5.1 to 8.5.3.

\subsubsection{Total Loss of Actuator}

For the total loss of effectiveness scenario, all three actuators fail from $0.3 T$ (2380 s) to $1 T(7934 \mathrm{~s})$ in the simulation. These faults are graphically shown in Fig. 8.11. A value of 1 indicates healthy actuators and 0 indicates the total loss of effectiveness. Figure 8.12 shows the position error of the system for 1.5 orbits. As observed here, the error increases from $2380 \mathrm{~s}$ to $7934 \mathrm{~s}$ when the actuators are not working.

The recovery from the fault is presented in Fig. 8.13. It can be concluded from this graph that SAC has the fastest recovery from the faults and NTSMC has the slowest recovery rate.

The position error after recovery is shown in Fig. 8.14. SAC and OSMC have the lowest error. The results from this graph look similar to the ones for the nominal case steady state error, Fig. 8.4. This indicates that after the fault, the system recovers completely.
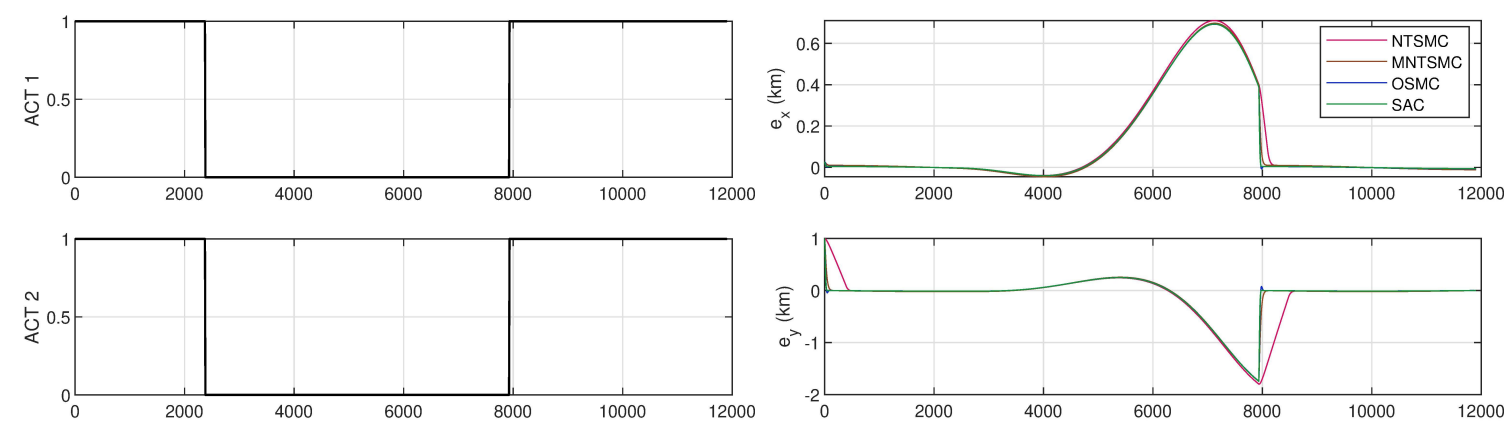

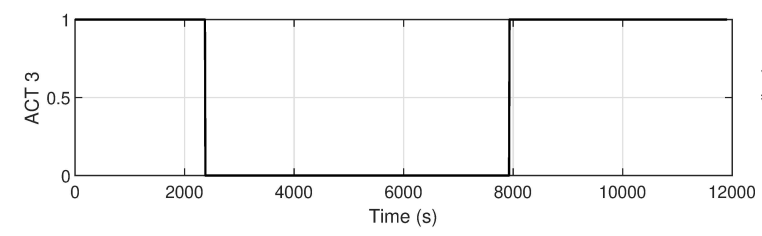

Figure 8.11: Faults Representation

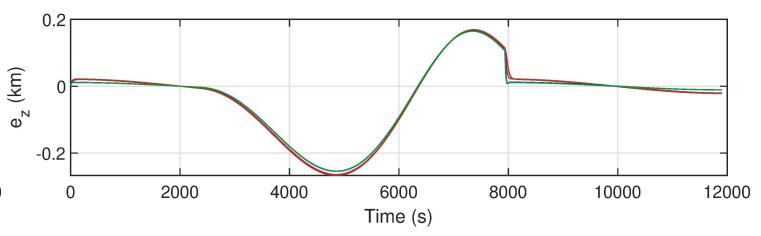

Figure 8.12: Position Error 

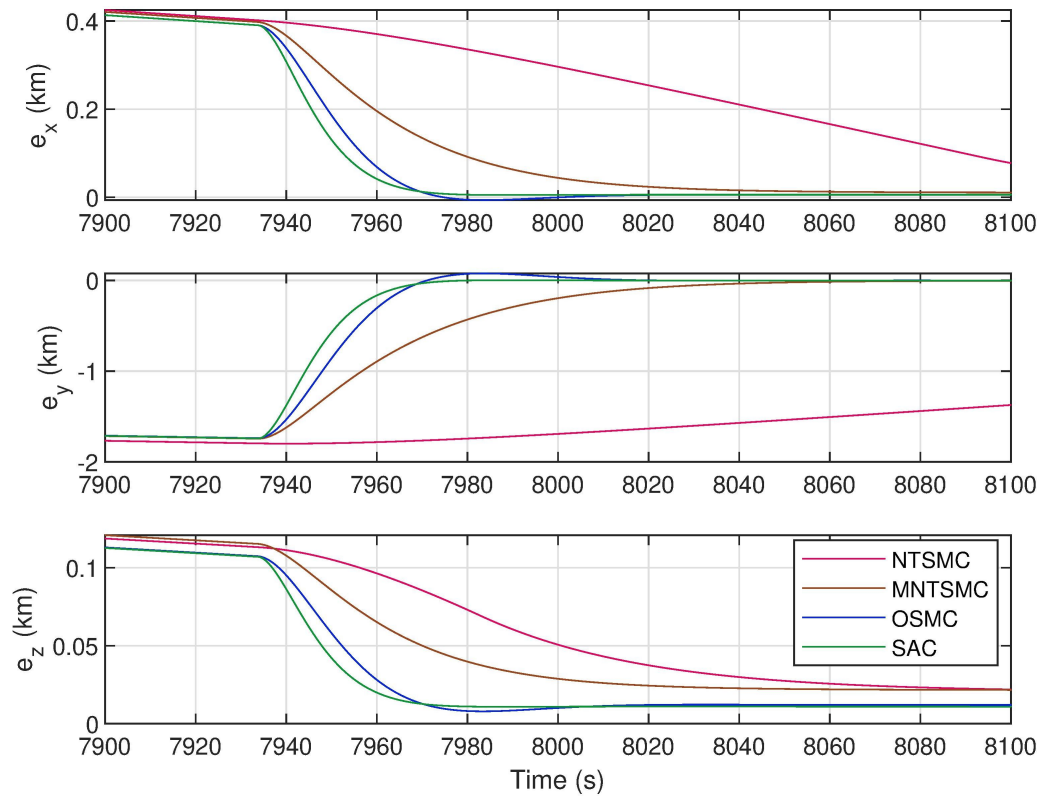

Figure 8.13: Position Error during Recovery
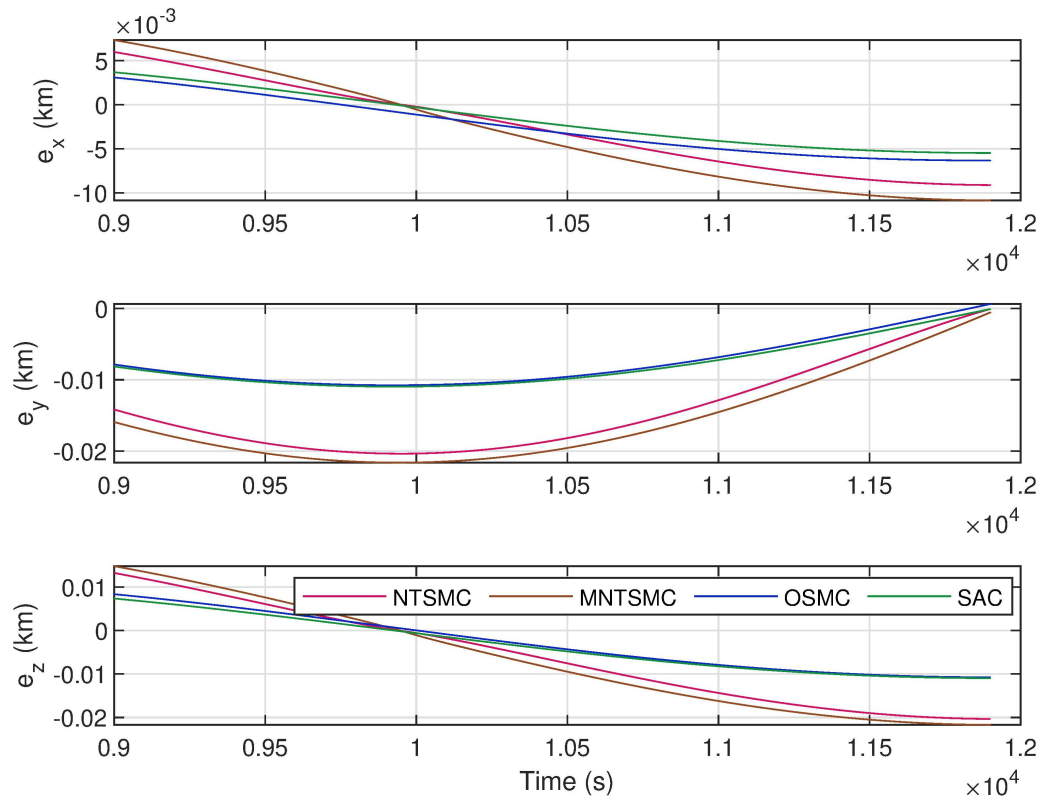

Figure 8.14: Position Error after Recovery

The control force for the system is presented in Fig. 8.15. It can be observed from this graph that SAC requires the largest force during recovery. Looking at the recovery time frame more closely in Fig. 8.16, it can be concluded that even 
though SAC requires the largest force, it is not much greater than the one required by OSMC.
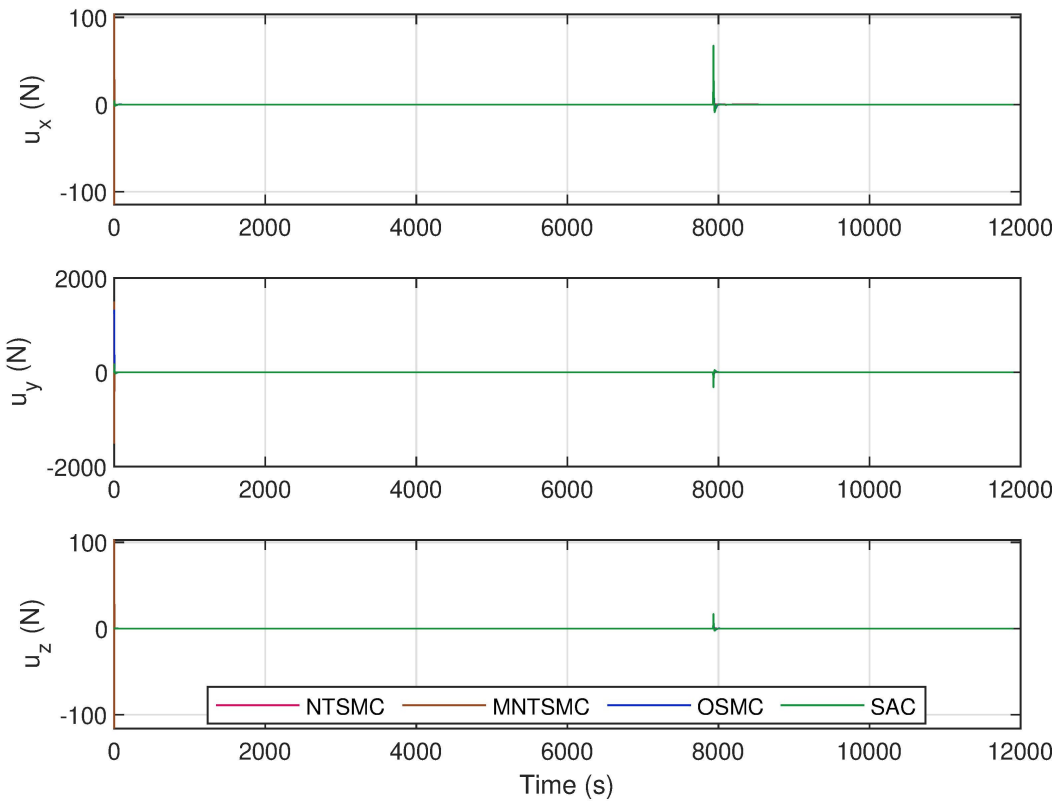

Figure 8.15: Control Force
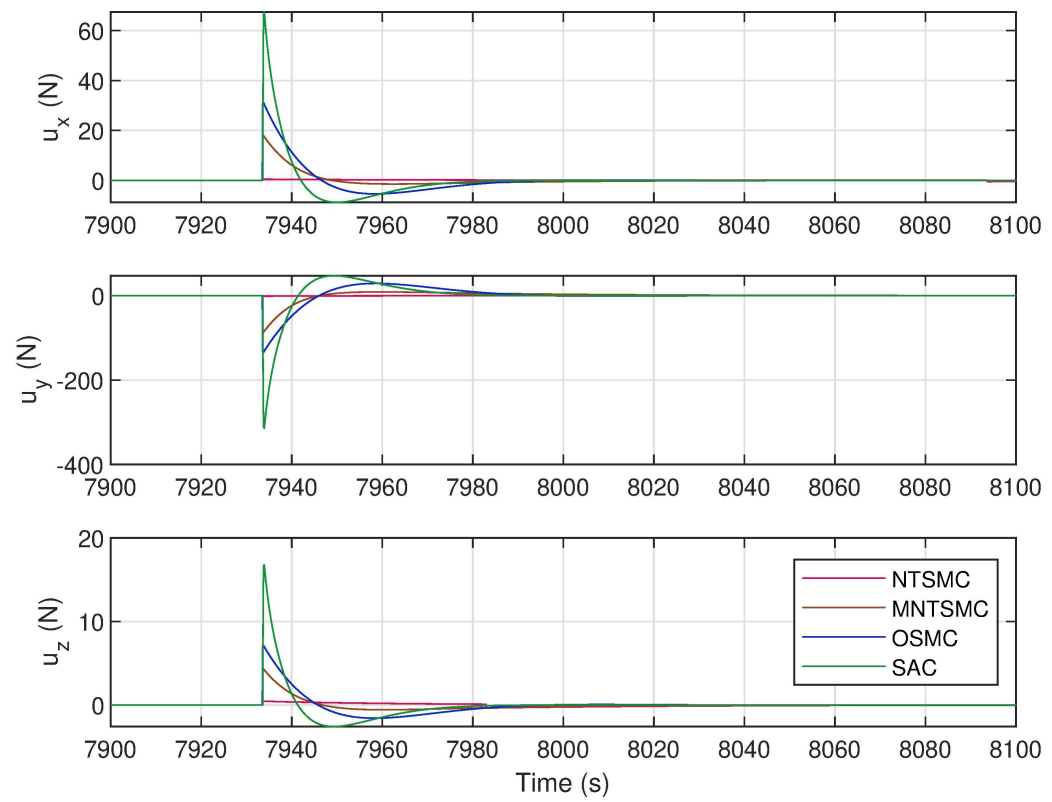

Figure 8.16: Control Force during Recovery 


\subsubsection{Locked in Place}

This section highlights the results for the locked in place scenario. The three actuators are stuck at $\left[-2.2 \times 10^{-4},-5.7 \times 10^{-4},-1.36 \times 10^{-3}\right]^{T} \mathrm{~N}$ from $0.3 T(2380 \mathrm{~s})$ to $1.3 T(10314 \mathrm{~s})$, that is for a duration of one orbit. These values are chosen since they are on average the values of the actuators before the fault begins. These values are shown in Fig. 8.17. A fixed value was selected so that the position error and the control force would be the same for all control laws during the fault time frame, allowing for a better comparison of the results.
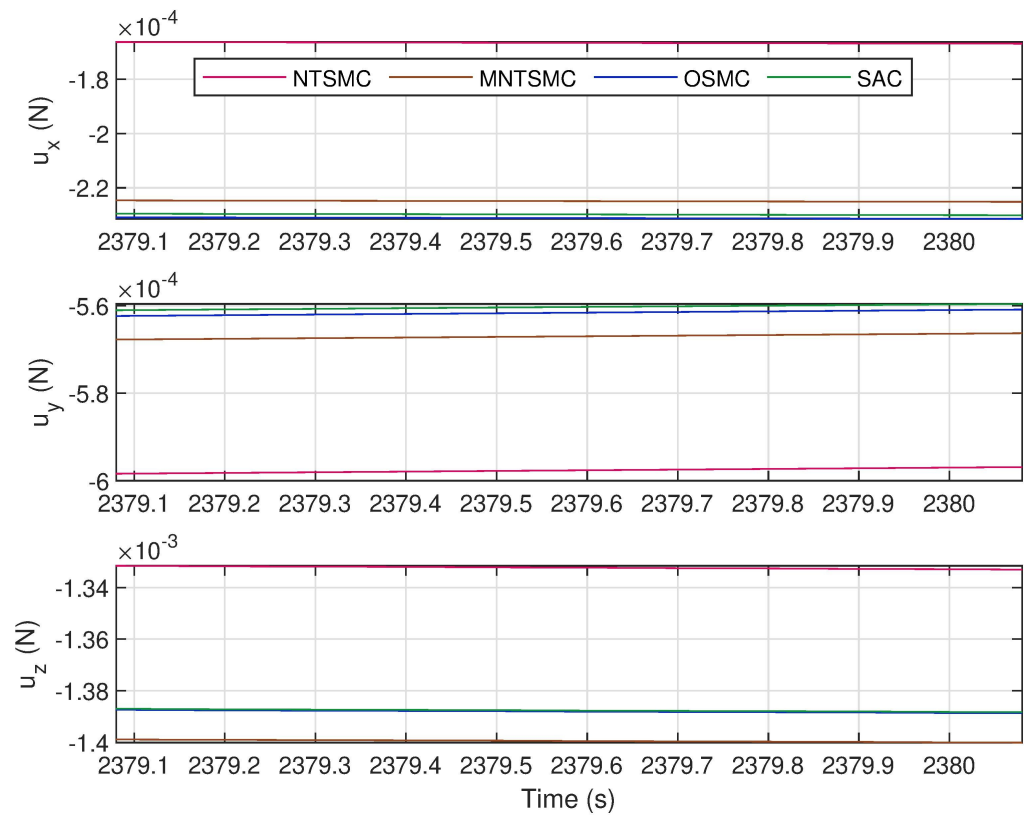

Figure 8.17: Actuator Forces before Faults

The faults are shown in Fig. 8.18, where 1 indicates healthy actuators and -1 presents the time during which the actuators are stuck. Figure 8.19 shows the position error. During the fault, the position error increases in all three axis, and the largest error is observed in the y-axis. As seen here, NTSMC has the slowest recovery rate compared to the three other control laws.

Figure 8.20 shows the position error during the recovery time frame. SAC has the fastest recovery rate, with OSMC not being far behind it; however, OSMC experiences an overshoot. This is significantly experienced in the $\mathrm{x}$-axis.

Figure 8.21 presents the position error after the recovery from the faults. All control laws except NTSMC have recovered from the fault and have a small steady state error, with SAC and OSMC consistently having the smallest error along the three axes. 

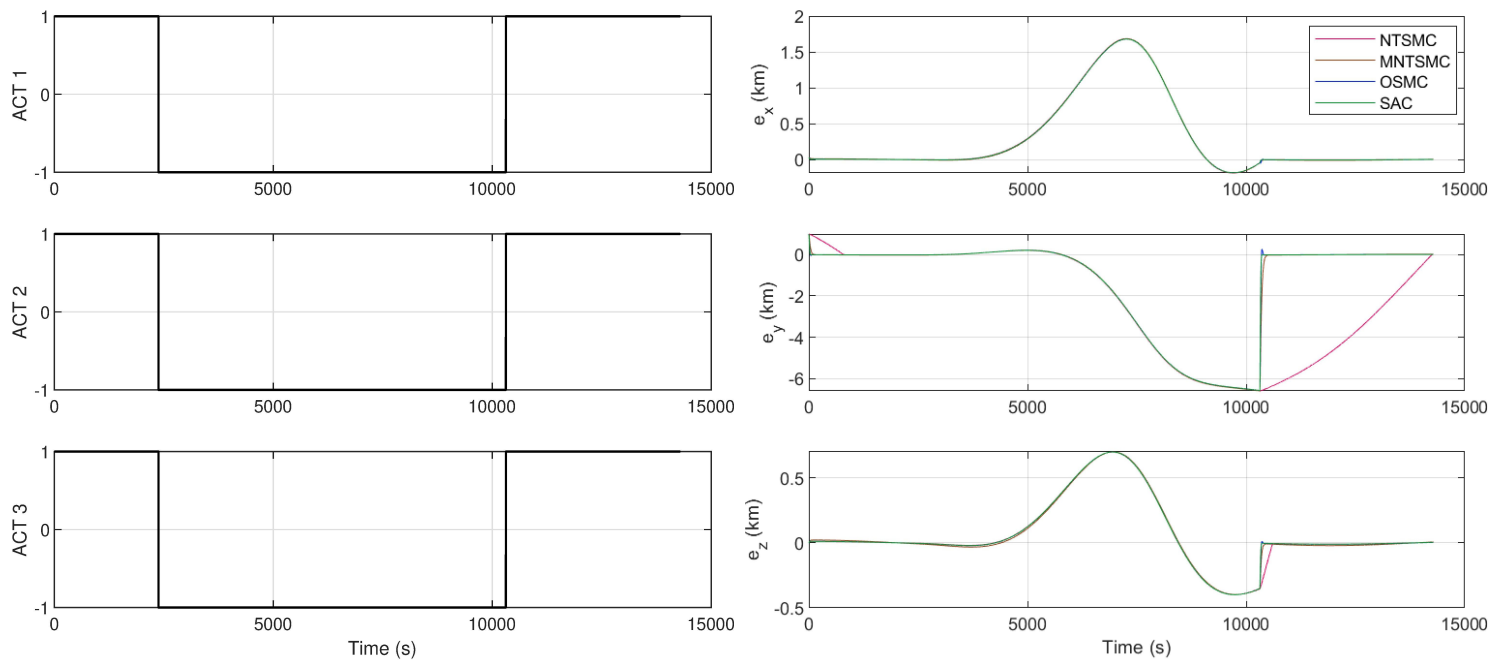

Figure 8.18: Faults Representation

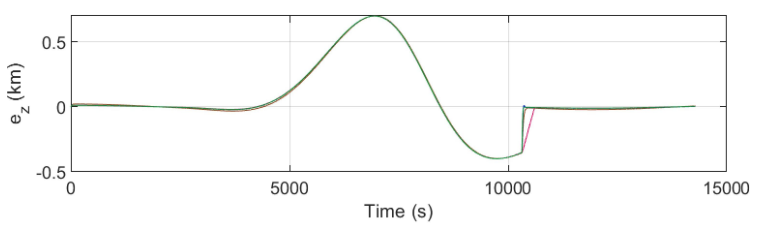

Figure 8.19: Position Error
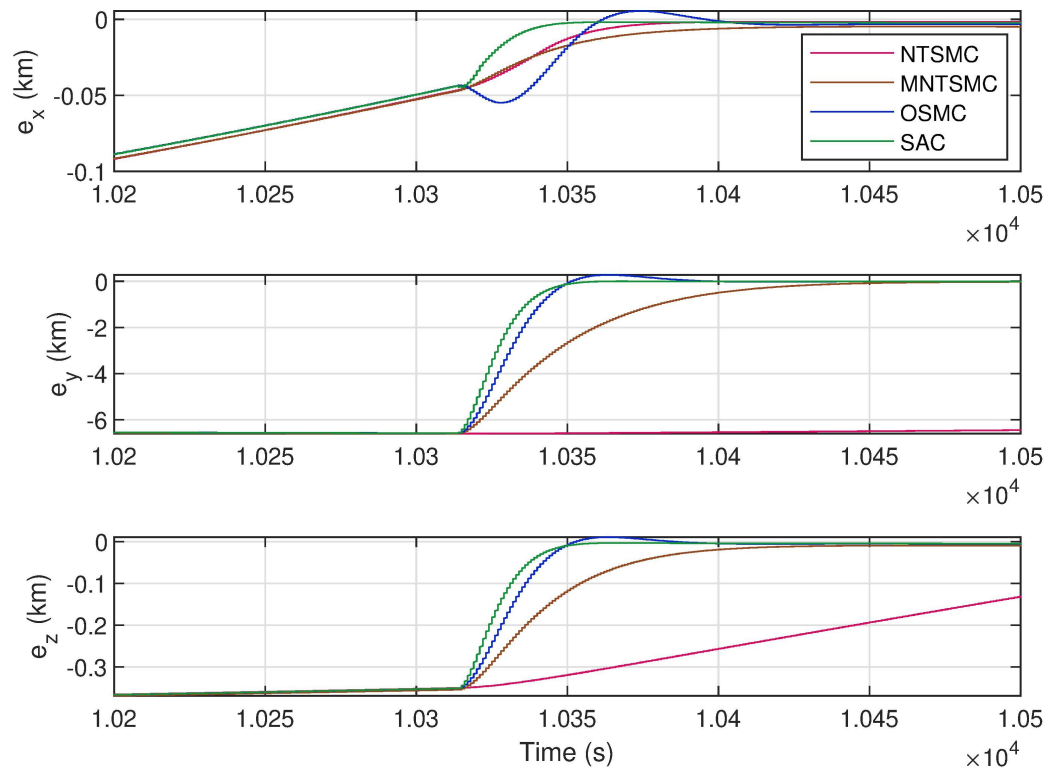

Figure 8.20: Position Error during Recovery 

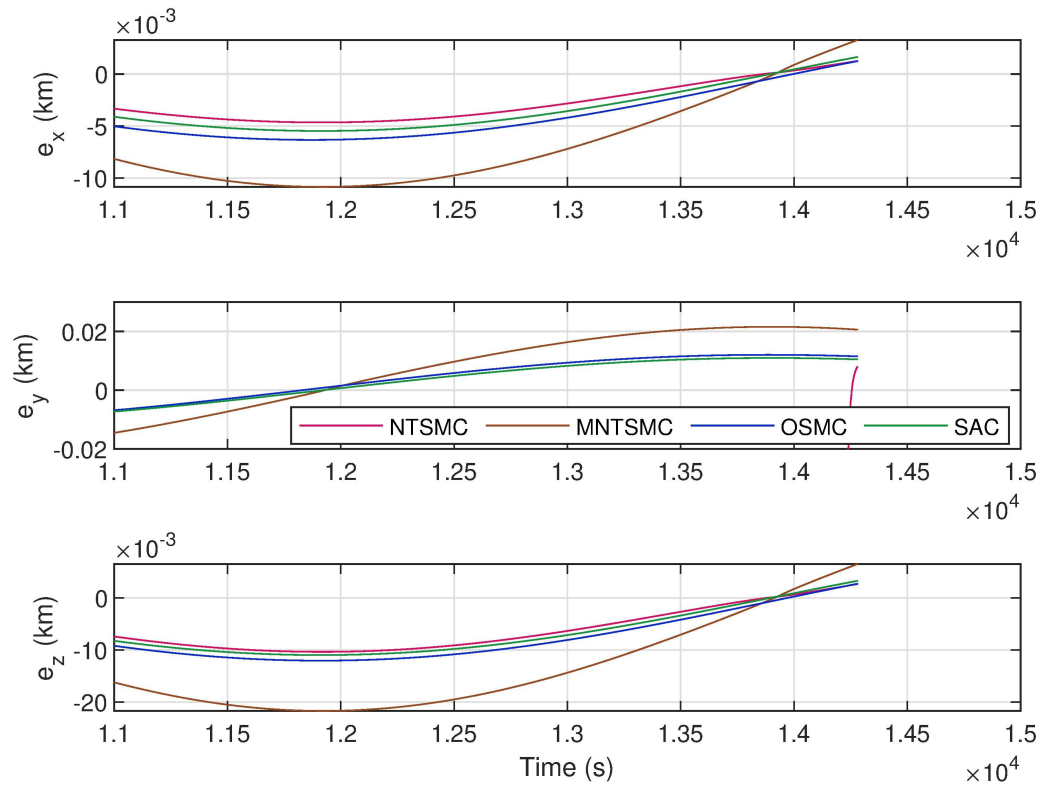

Figure 8.21: Position Error after Recovery

The control force for the system is presented in Fig. 8.22. It can be observed from this graph that SAC requires the largest force during recovery. Figure 8.23 shows the control force required during recovery in more details. Even though SAC has the fastest convergence rate, it comes at the cost of increased control efforts. The presence of the sliding surface allows for the SMC laws to converge faster and require less control force when the tracking error is large. This is why in Fig. 8.23, $\mathrm{SAC}$ requires significantly more control force than the other three control laws. 

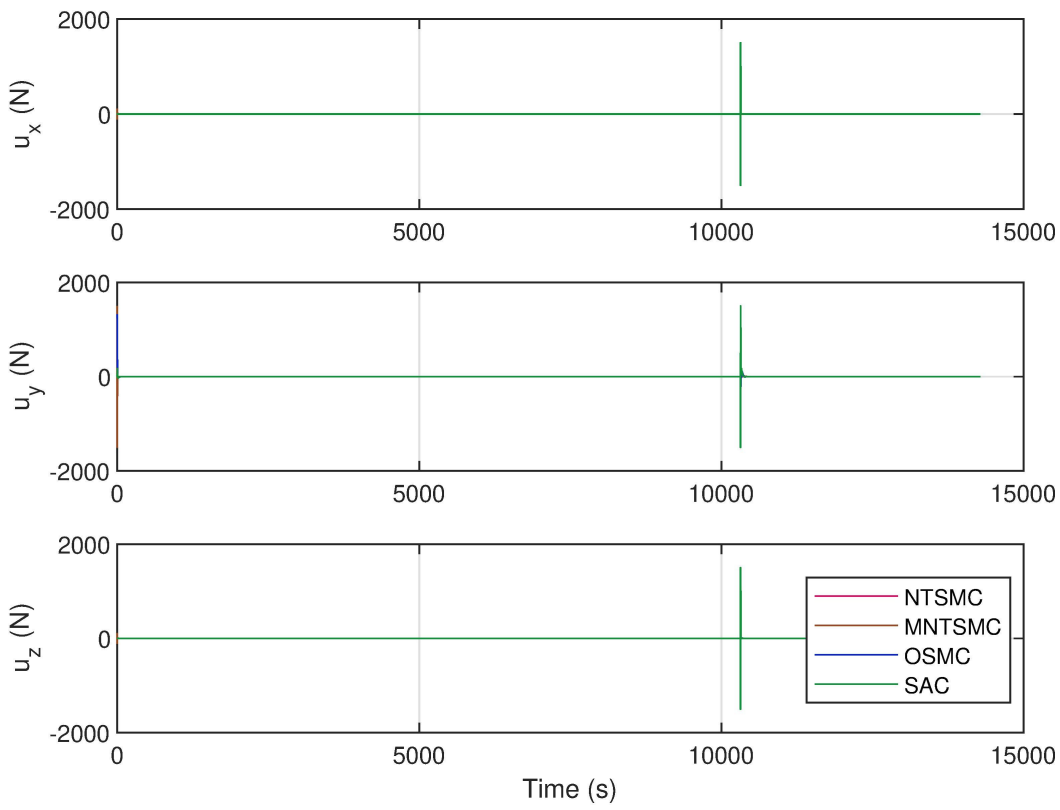

Figure 8.22: Control Force
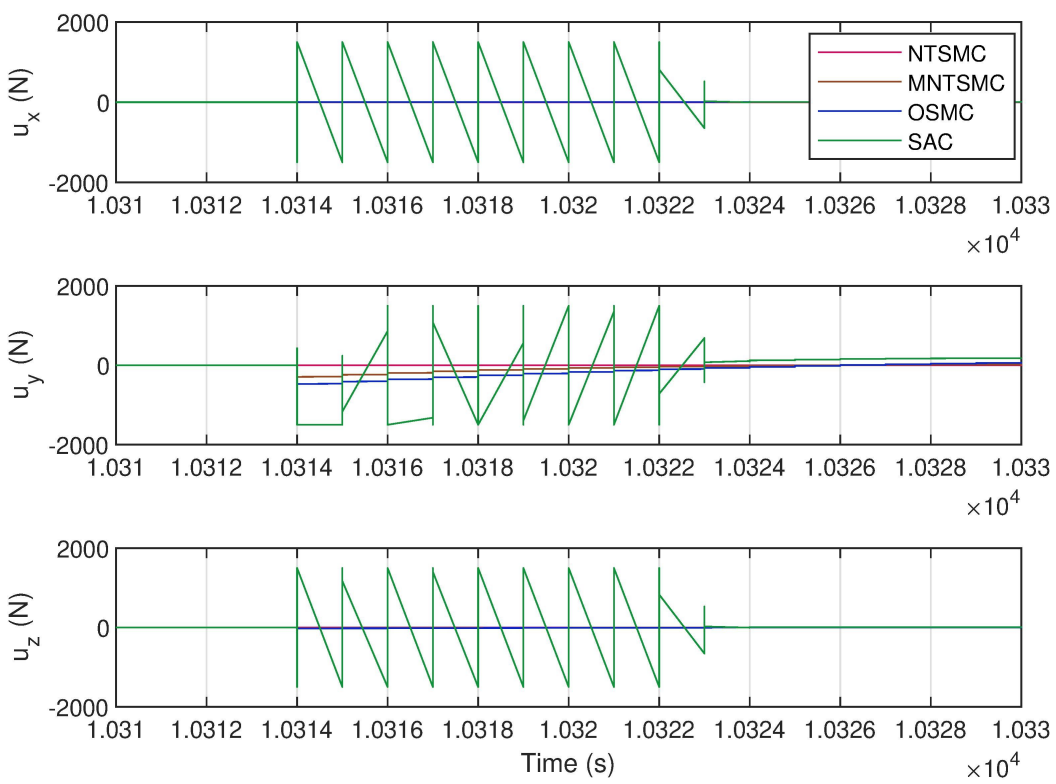

Figure 8.23: Control Force during Recovery 


\subsubsection{Partial Loss of Effectiveness}

This section presents the results for partial loss of effectiveness. The effectiveness of all three actuators is lost by $95 \%$ from $30 \mathrm{~s}$ to $0.5 T$. The loss of effectiveness is shown in Fig. 8.24 and the position error of the system is shown in Fig. 8.25. The position error during the fault time frame is presented in Fig. 8.26. Based on these results, it can be seen that $\mathrm{SAC}$ does not get affected by the faults and has the best performance.
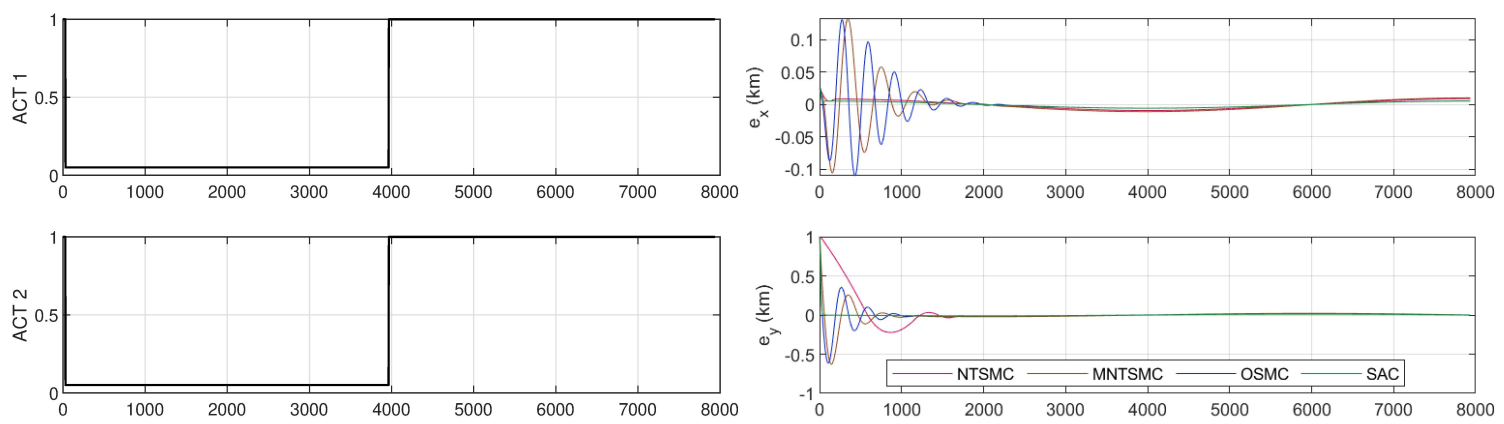

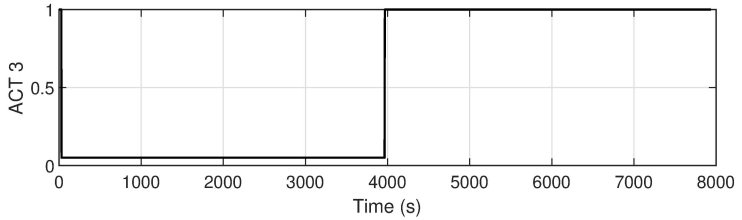

Figure 8.24: Faults Representation

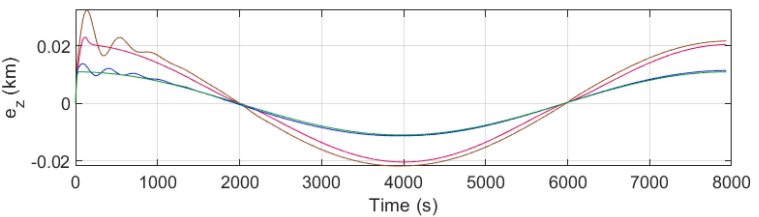

Figure 8.25: Position Error 

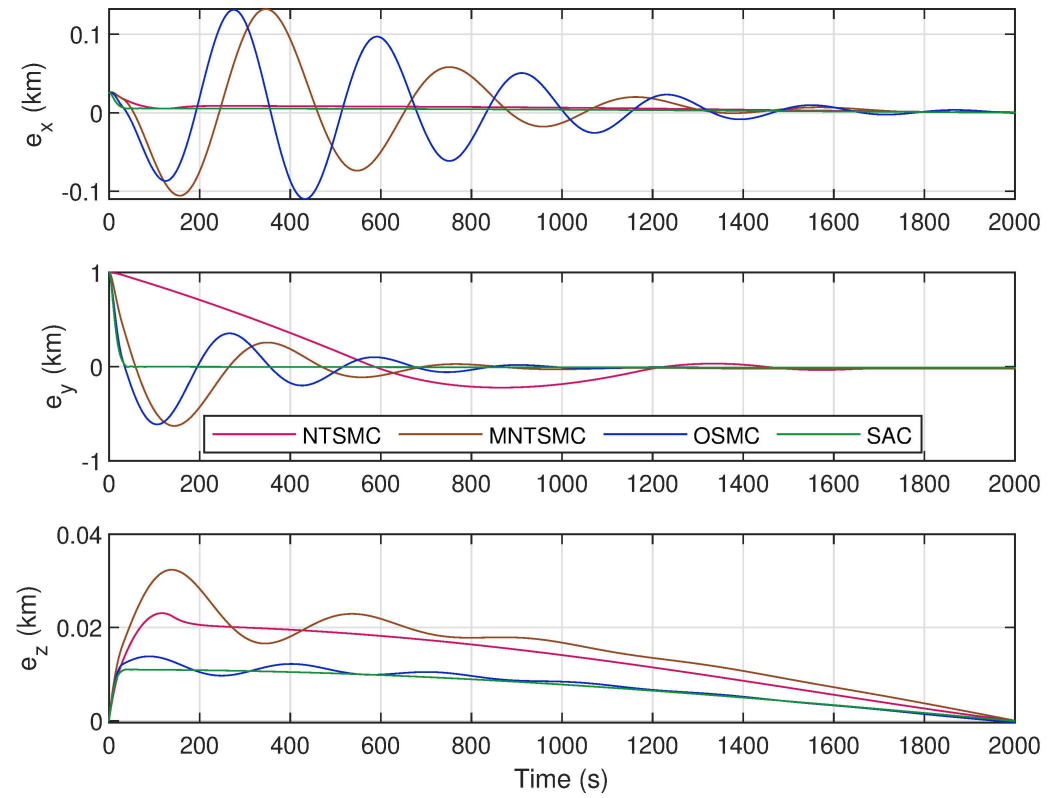

Figure 8.26: Position Error during Fault

Figure 8.27 presents the overall control force of the system and Fig. 8.28 presents the control force for the first 0.25 seconds. The control force does not get affected by the faults and looks identical to the nominal scenario.
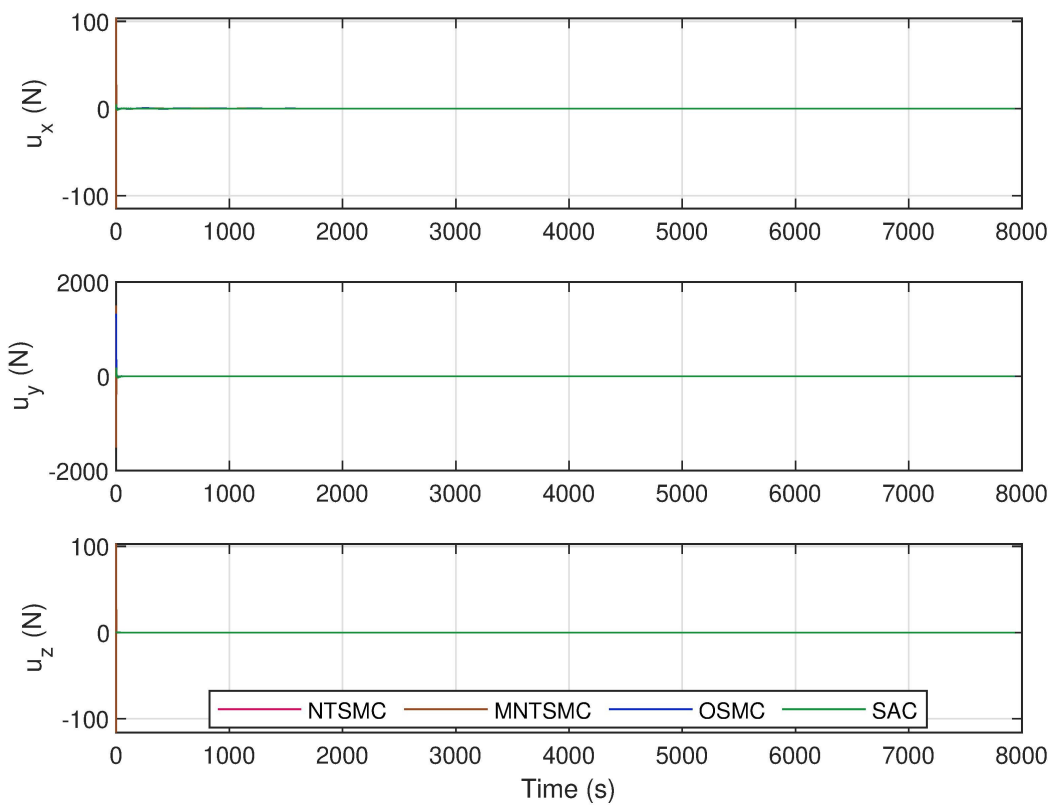

Figure 8.27: Control Force 

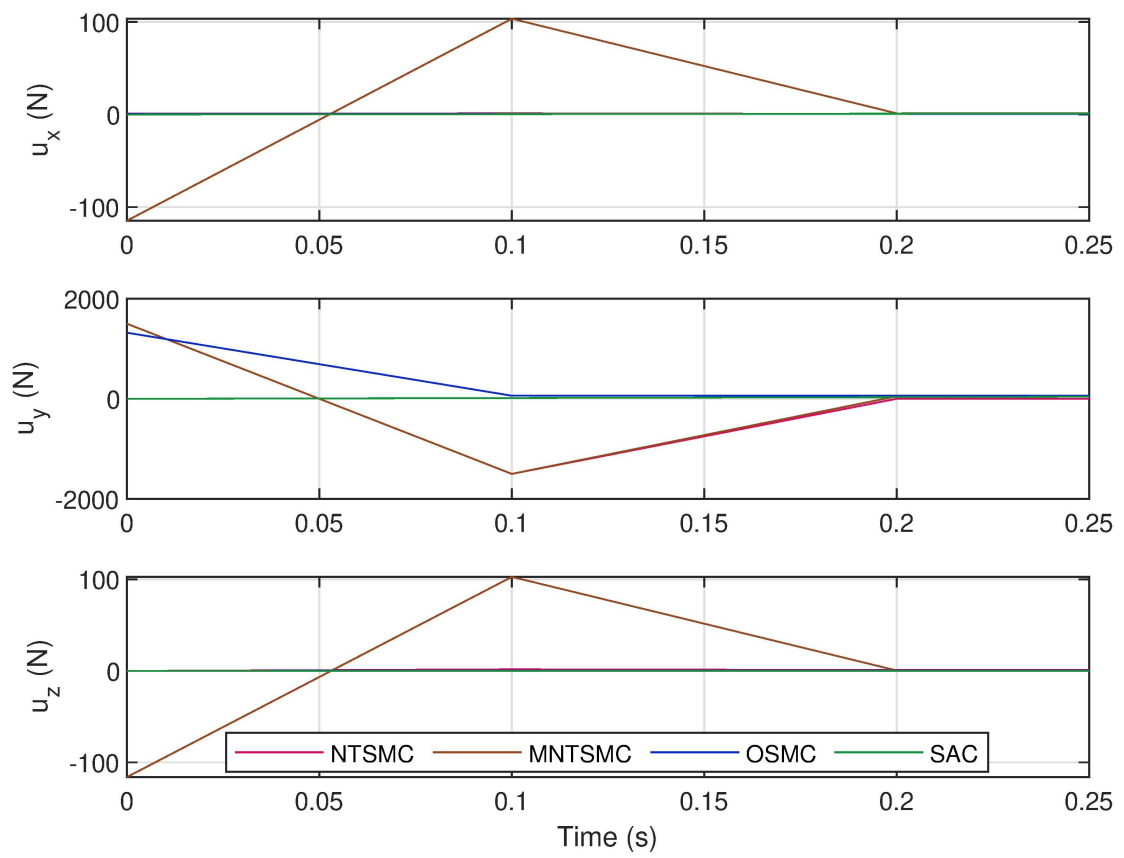

Figure 8.28: Control Force during Fault 


\subsubsection{Ideal Model Reset for SAC}

The ideal model for SAC was reset for two of the fault scenarios: the total loss of effectiveness and the locked in place scenario. This means the initial condition of the integration in the ideal model was updated continuously during the fault. More specifically, for $\dot{\boldsymbol{x}}_{m}=\boldsymbol{A}_{m} \boldsymbol{x}_{m}+\boldsymbol{B}_{m} \boldsymbol{u}_{m}$, the initial condition for the integration of $\dot{\boldsymbol{x}}_{m}$ was updated throughout the fault based on the current $\boldsymbol{x}$ matrix, which includes the position and velocity components of the chaser, from the plant. The graphical representation of this is shown in Fig. 8.29. The reset causes the value of $\boldsymbol{e}_{y}$ to stay very small, thus improving the performance of SAC.

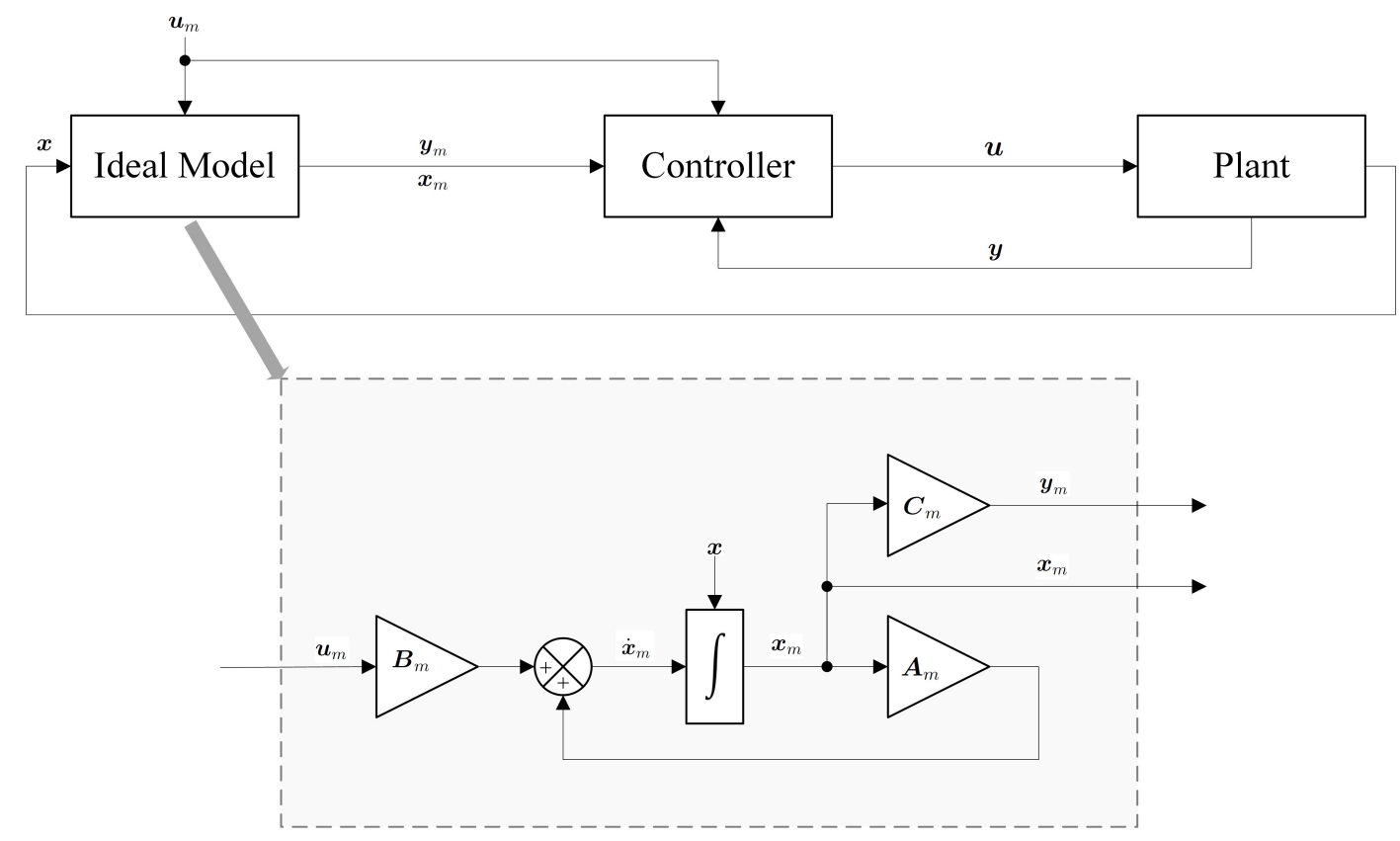

Figure 8.29: Ideal Model with Reset

The comparison of the results with and without resetting the ideal model are presented in Figs. 8.30 to 8.33 for the total loss scenario and in Figs. 8.34 to 8.37 for the locked in place scenario.

As seen in Fig. 8.30, without resetting, the system experiences some overshoot. Figure 8.31 shows the position error during the recovery time frame; the recovery is faster for the case without a reset at the cost of significant overshoot. In terms of control force, as observed in Figs. 8.32 and 8.33, the system requires more control force without the reset. 

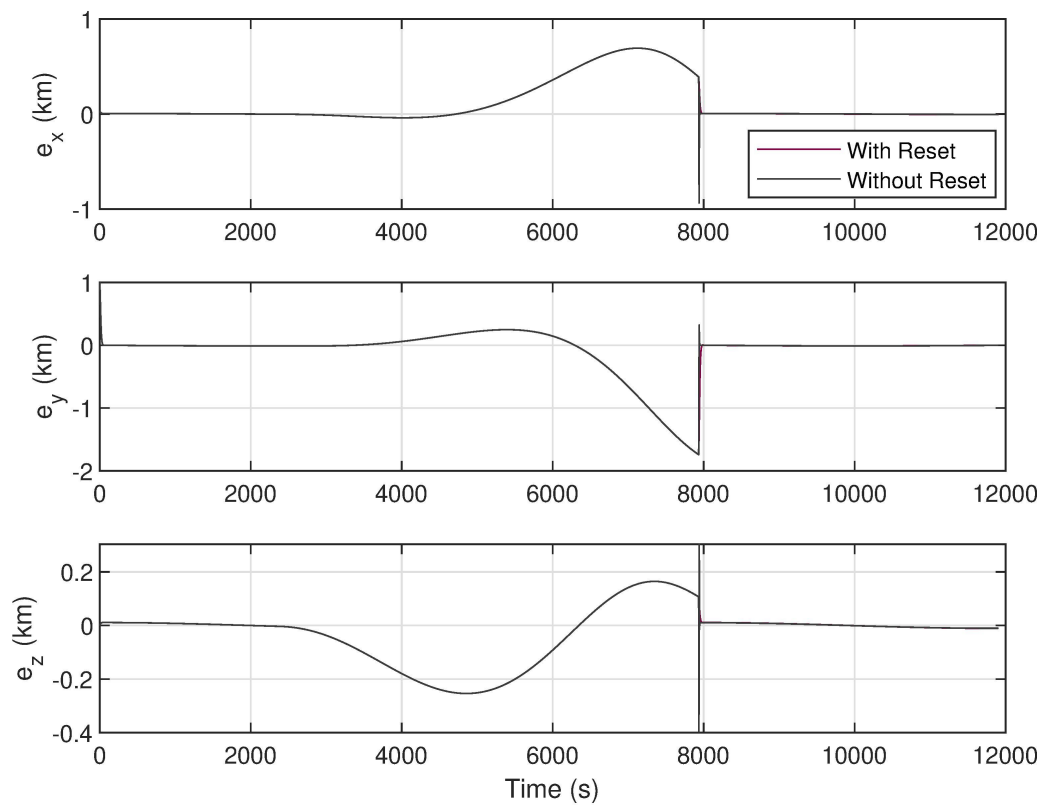

Figure 8.30: Position Error for the Total Loss Scenario
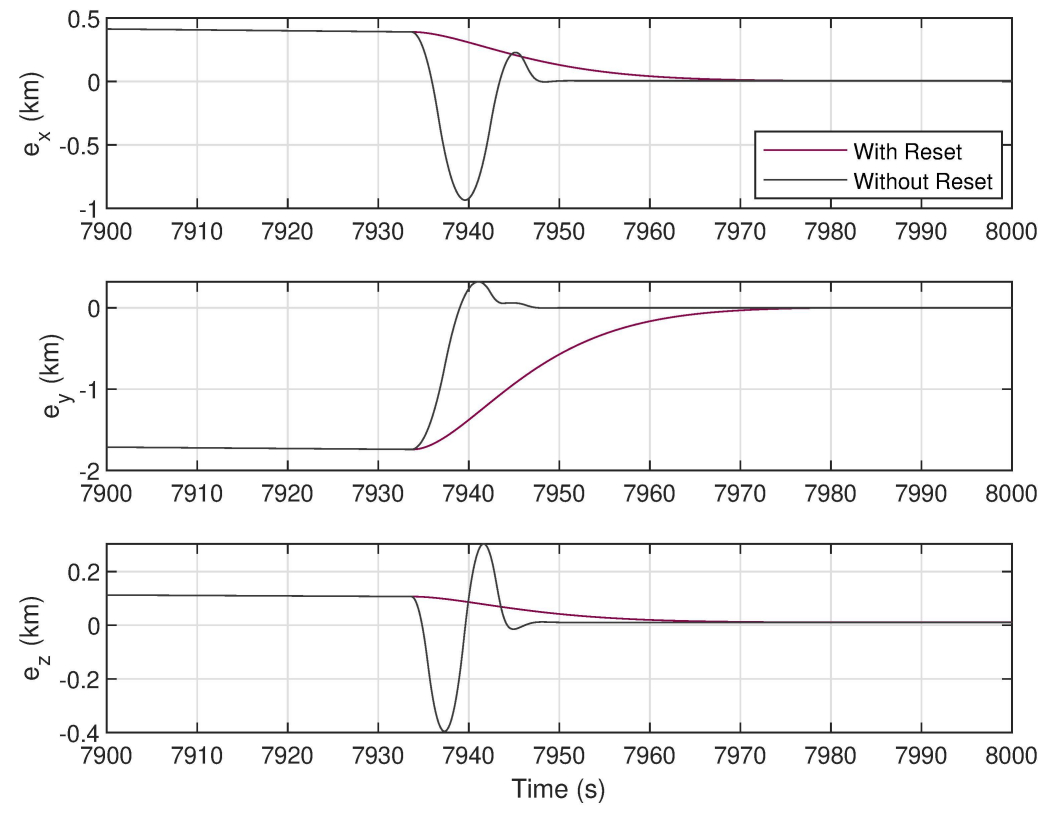

Figure 8.31: Position Error Recovery for the Total Loss Scenario 

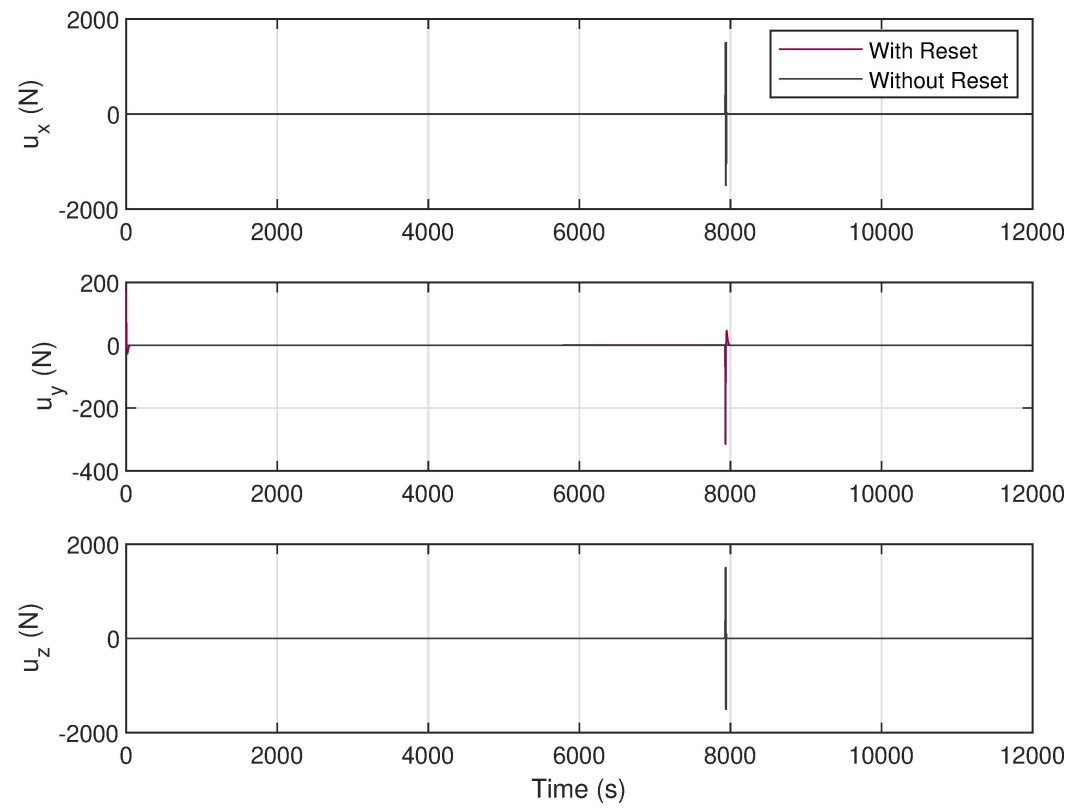

Figure 8.32: Control Force for the Total Loss Scenario
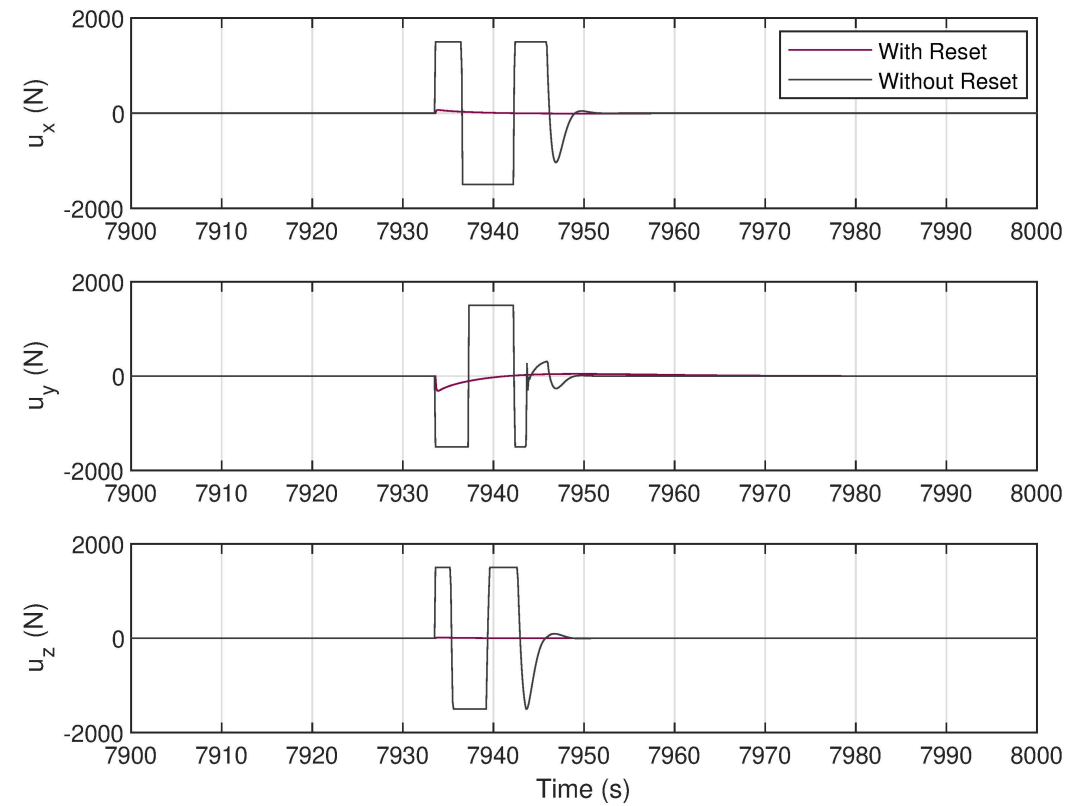

Figure 8.33: Control Force during Recovery for the Total Loss Scenario 
When the position error is substantial, as seen in Fig. 8.34, without resetting the ideal model, the system experiences more overshoot compared to the total loss scenario. Resetting the ideal model yields significantly better results during the recovery of the position error as observed in Fig. 8.35.

In terms of control force, the z-axis for the locked in place scenario requires a tremendous amount of control force, as shown in Fig. 8.36. Looking more closely at the recovery time frame in Fig. 8.37, it can be concluded that without resetting the ideal model, the performance of SAC will degrade significantly. Therefore, the conclusion is made that for fault scenarios where the system experiences a large position error, resetting the ideal model greatly improves the performance of SAC.
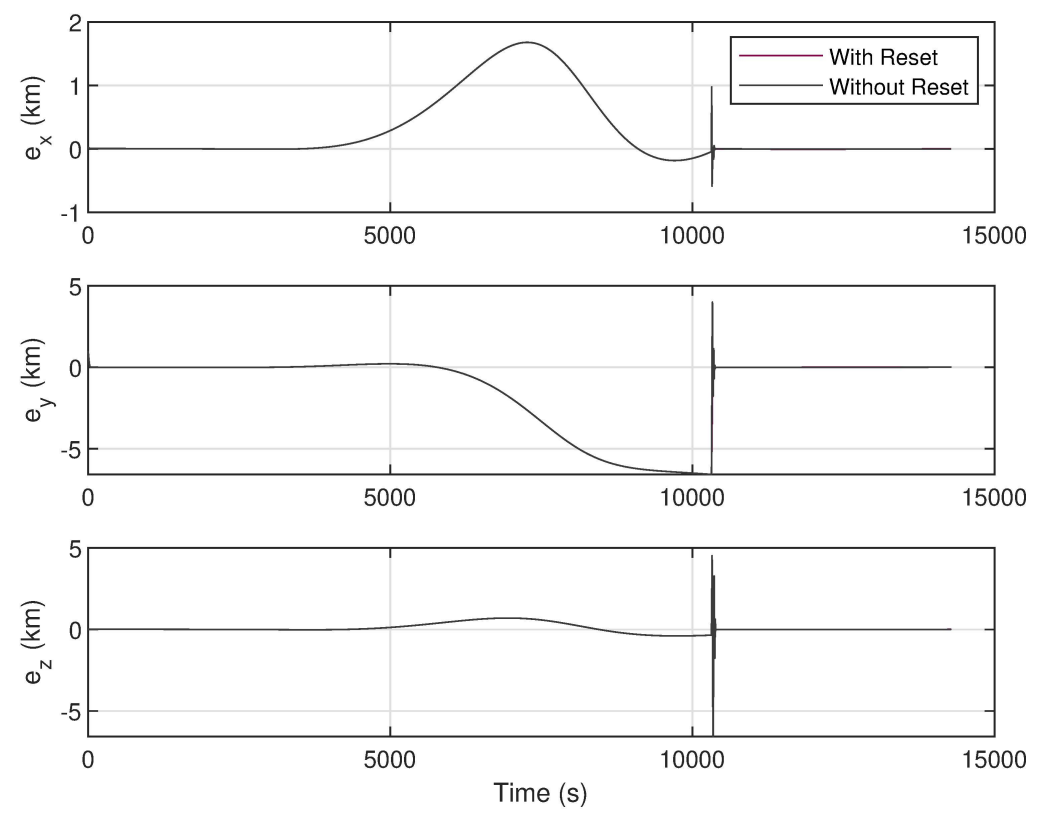

Figure 8.34: Position Error for the Locked in Place Scenario 

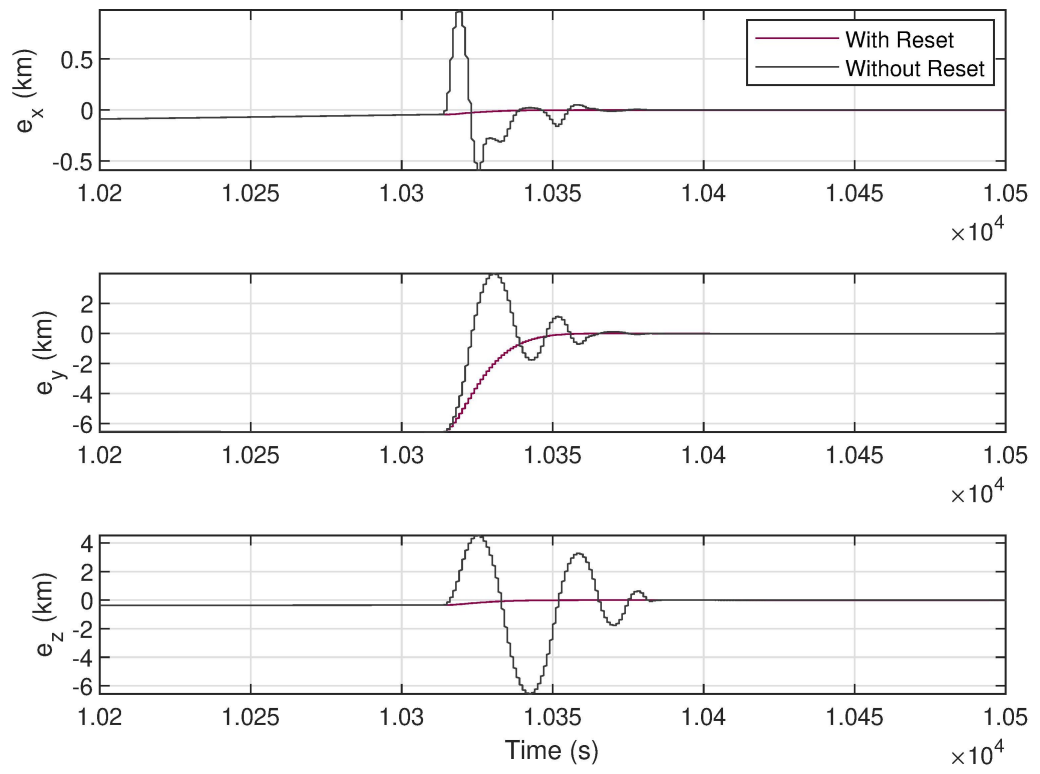

Figure 8.35: Position Error Recovery for the Locked in Place Scenario
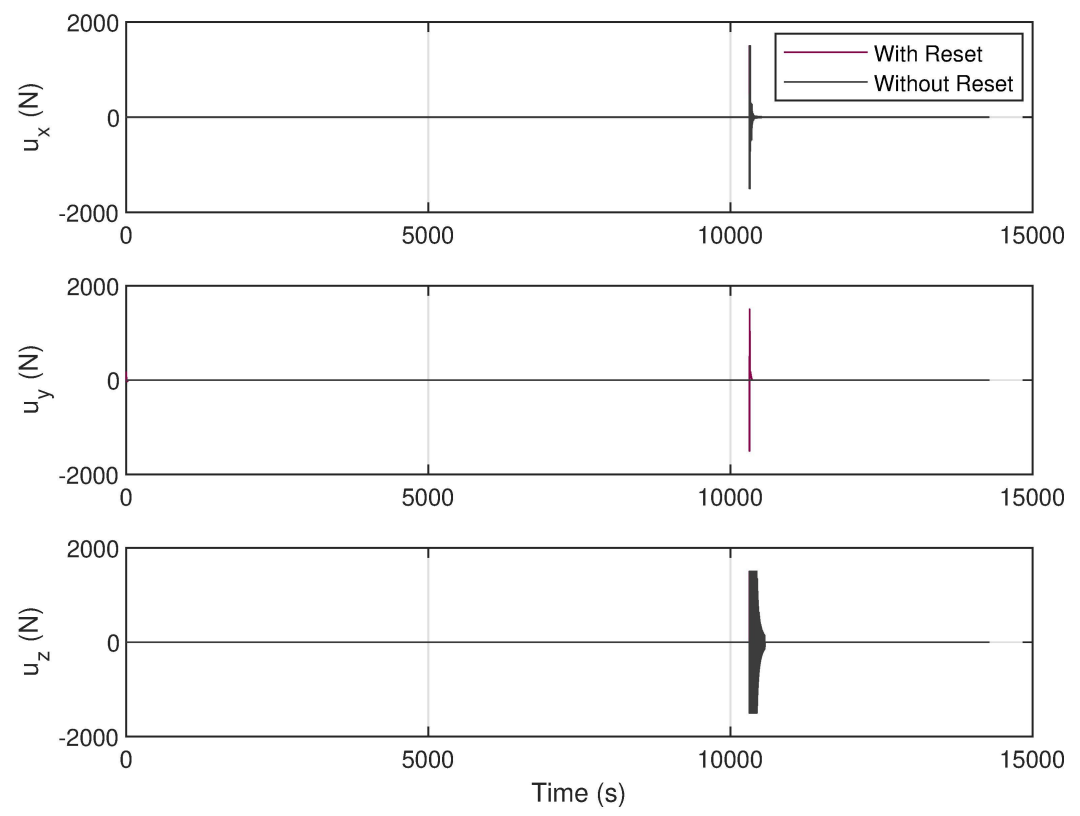

Figure 8.36: Control Force for the Locked in Place Scenario 

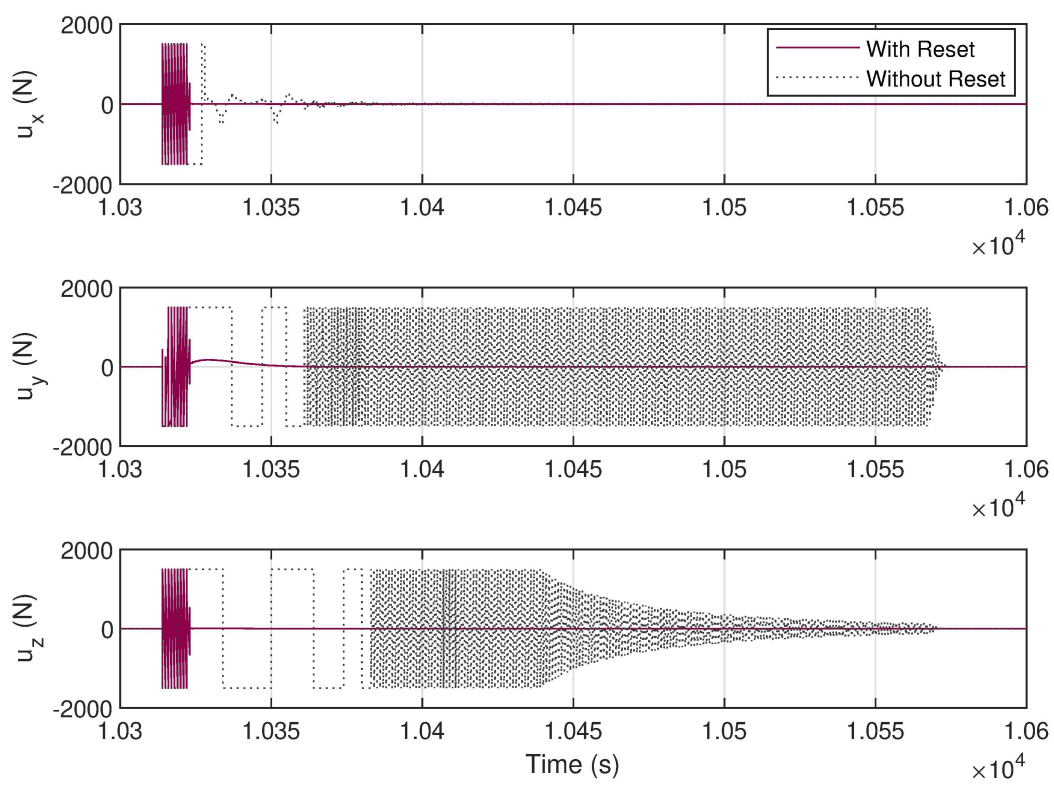

Figure 8.37: Control Force during Recovery for the Locked in Place Scenario 


\subsection{Control Allocation}

This section presents the results for CA. Two different fault scenarios are simulated so that a better conclusions can be made regarding the performance of the CA algorithms. These results are highlighted in Secs. 8.6.1 and 8.6.2.

To begin the implementation of CA in MATLAB/Simulink, first the control force, which is in the LVLH reference frame has to be converted to ECI reference frame. This is done using the position and velocity vectors of the target spacecraft, as previously explained in Sec. 2.5.3. Then the control force vector from ECI reference frame has to be converted to the body fixed reference frame, since the matrix $\boldsymbol{D}$ contains the position of the actuators in the body fixed reference frame. The conversion from ECI to body fixed reference frame is done using the attitude kinematics and dynamics, explained in Sec. 2.3. Once the control allocation is performed, the control force has to be converted back to ECI reference frame since the calculations in the plant are in ECI. This is graphically shown in Fig. 8.38.

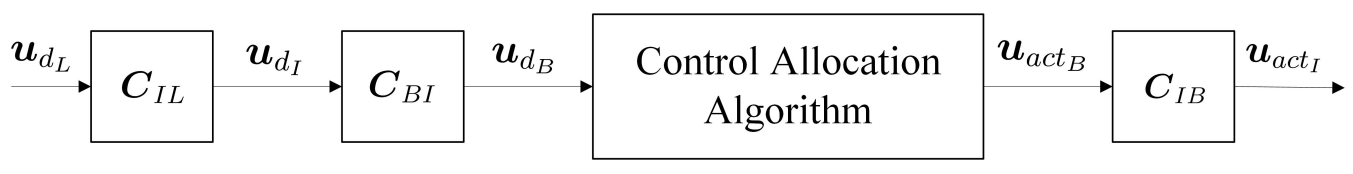

Figure 8.38: CA Reference Frames

\subsubsection{Scenario 1}

This subsection presents the results for the first scenario. Figure 8.39 presents the overview of the faults the system experiences in scenario 1 and Fig. 8.40 presents the overview of the actuator forces.

Figures 8.41 and 8.42 highlight the faults and the actuator forces during the fault time frame. As observed here, CLCA and MCLCA both have a smoother performance compared to WPI. WPI experiences spikes when the system undergoes faults.

A closer look at one of the spikes in Fig. 8.43 shows the amount of spike WPI experiences compared to CLCA and MCLCA.

The overall control force of the system in LVLH in shown in Fig. 8.44 and the forces during the fault are provided in Fig. 8.45. As seen here, the system has a smoother behaviour when CLCA and MCLCA algorithms are used.

Figure 8.46 presents the position error. All three CA algorithms result in the same position error. 

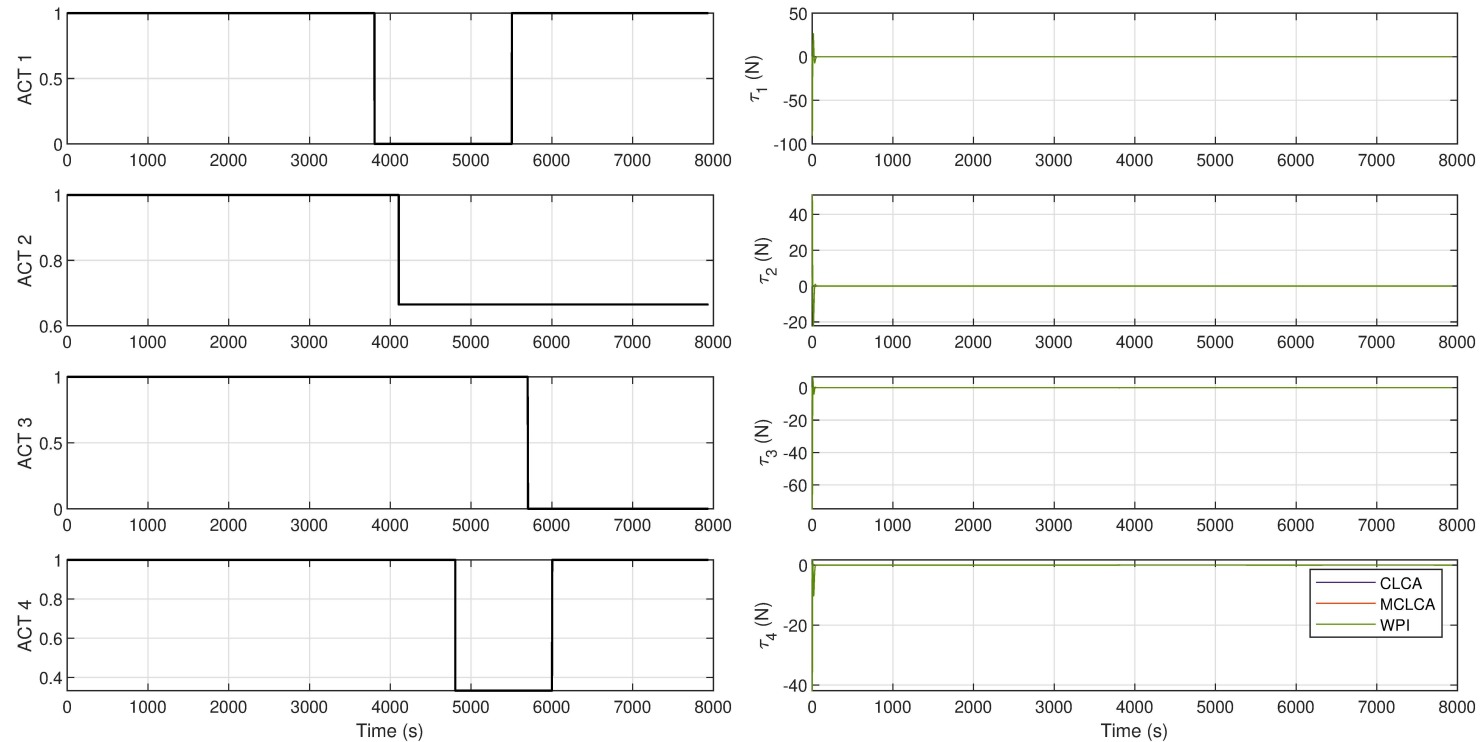

Figure 8.39: Fault Estimate

Figure 8.40: Actuator Forces
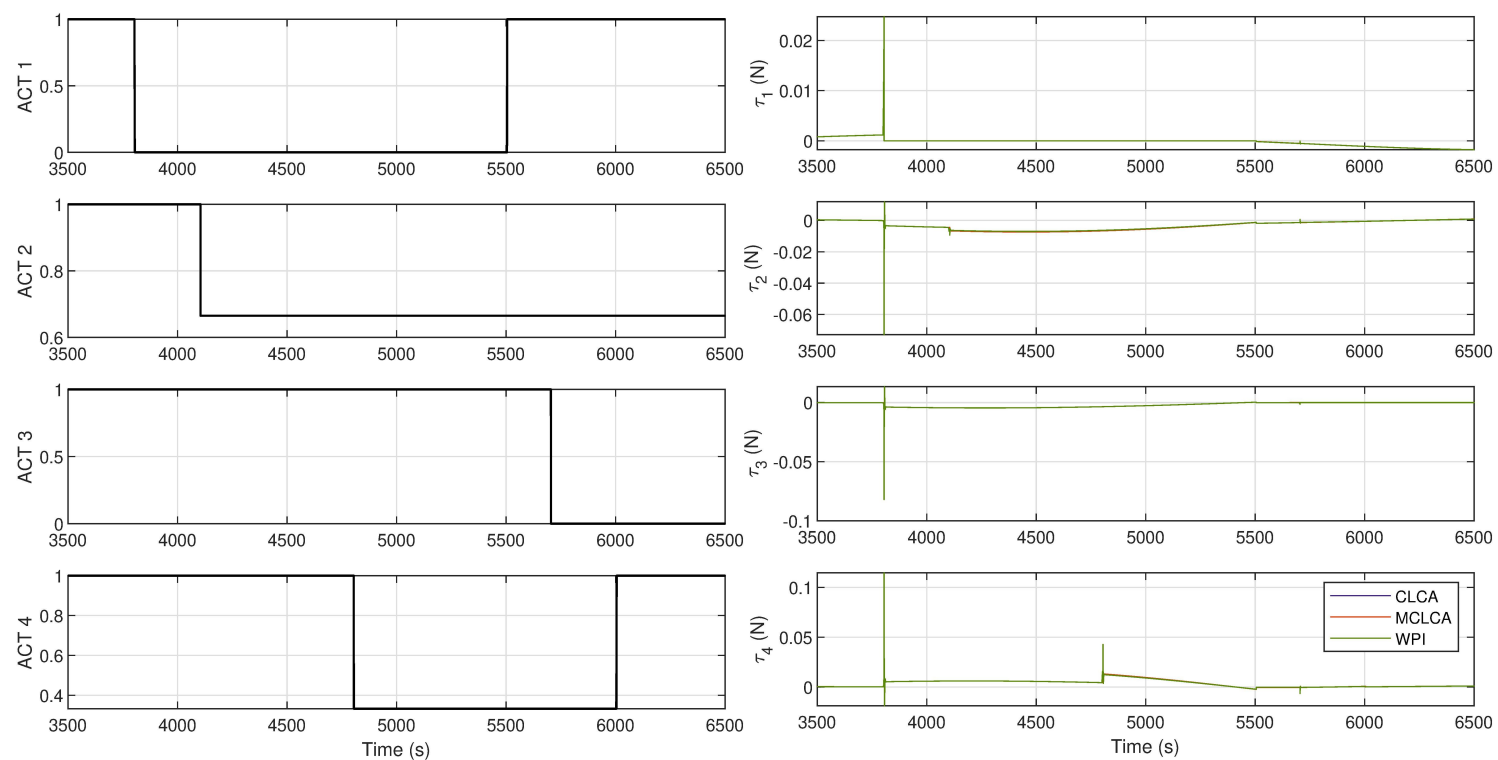

Figure 8.41: Fault Estimate

Figure 8.42: ACT Forces during Faults 

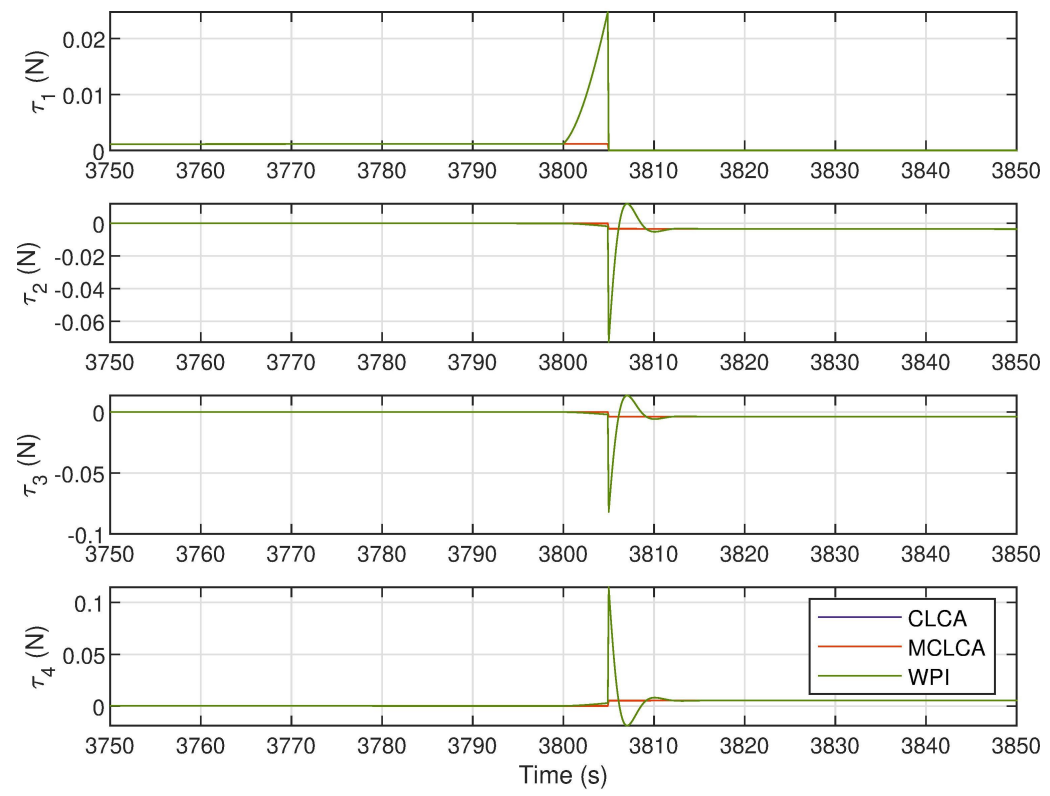

Figure 8.43: Actuator Force at 3805 seconds
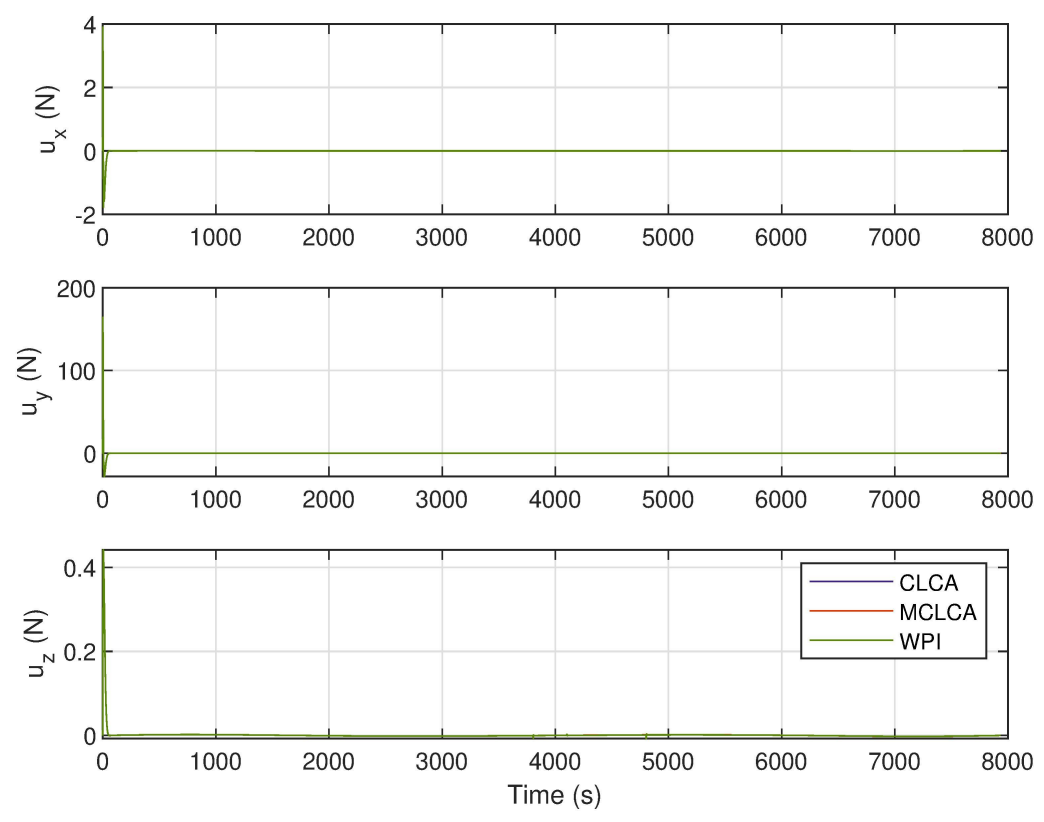

Figure 8.44: Control Force 

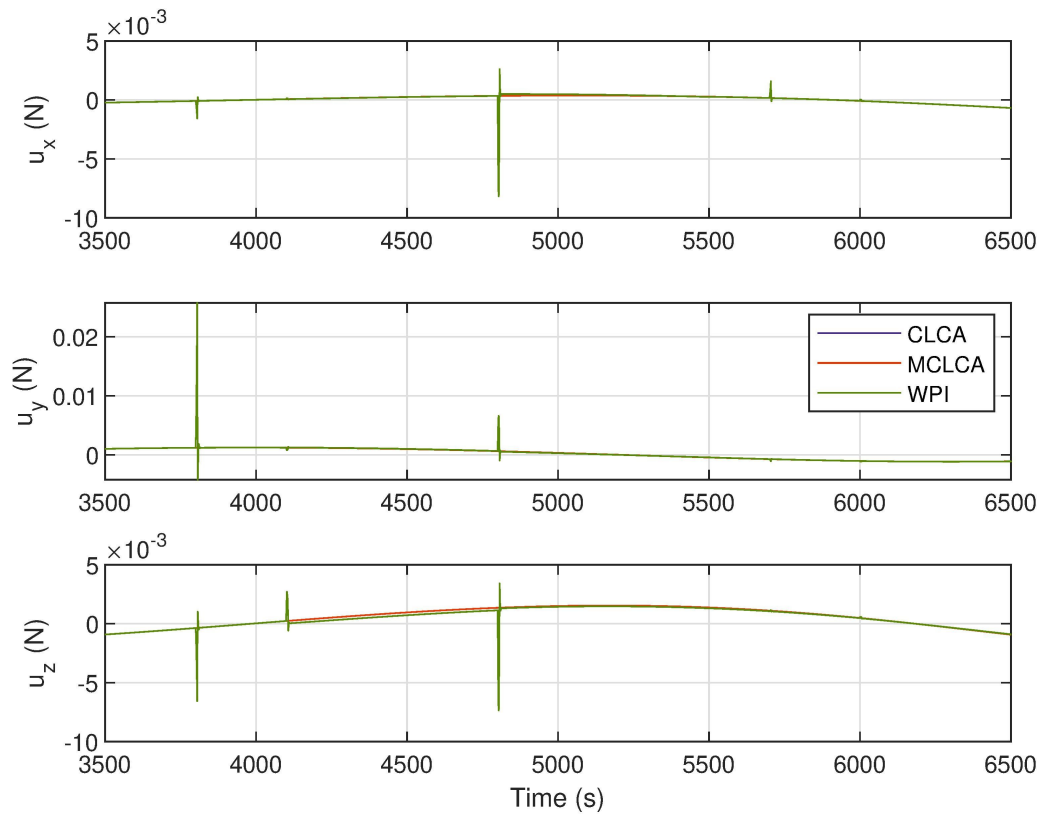

Figure 8.45: Control Force during Fault
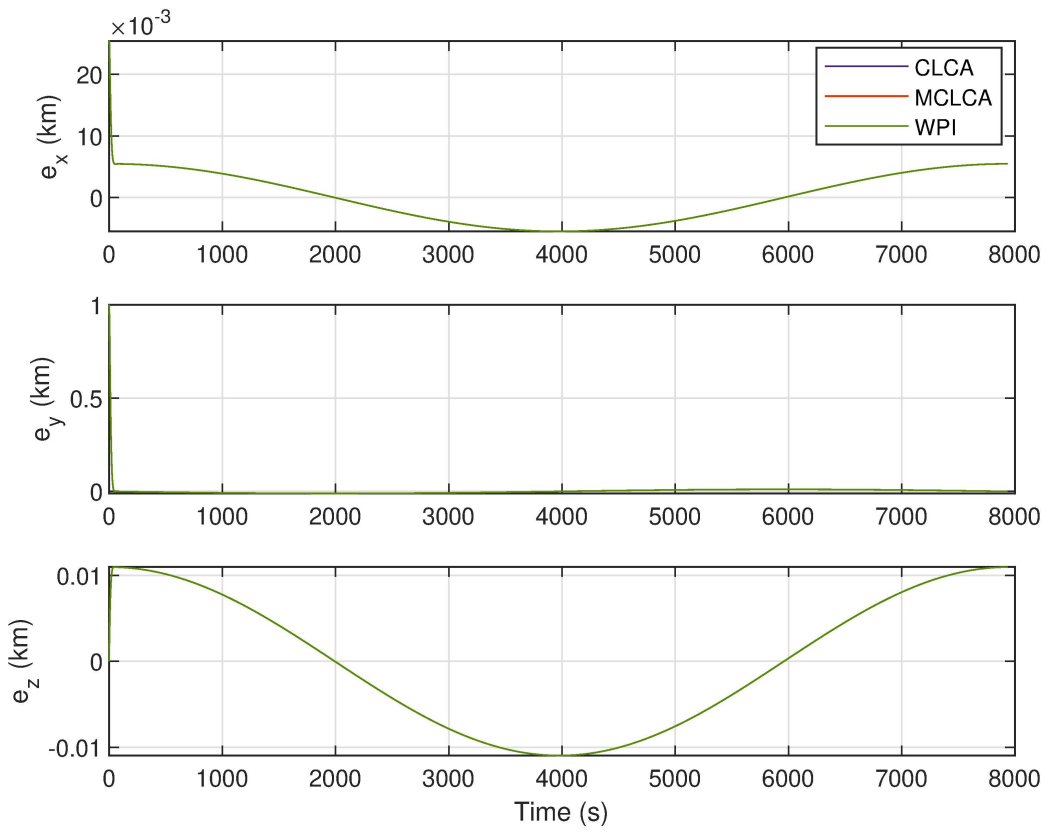

Figure 8.46: Position Error 


\subsubsection{Scenario 2}

This subsection presents the results for the second scenario. Compared to the first scenario, the faults that the system experiences in this scenario are more rigorous. The overall faults and actuator forces are presented in Figs. 8.47 and 8.48, respectively.

The faults and the actuator forces during the fault time frame are provided in Figs. 8.49 and 8.50, respectively. Given the severity of the faults, it can be observed that the performance of CLCA and MCLCA is more superior compared to scenario 1. WPI experiences much more spikes as the faults in the system change. A closer look at one of these spikes in shown in Fig. 8.51.

Figure 8.52 highlights the control force of the system for the duration of one orbit, while Fig. 8.53 shows the control force during the fault time frame. Similar to the first scenario, the performance of CLCA and MCLCA is better than WPI. Given that faults are more intense in this scenario, the spikes are worse.

In terms of the position error, the results is consistent for the three CA algorithms, as seen in Fig. 8.54.
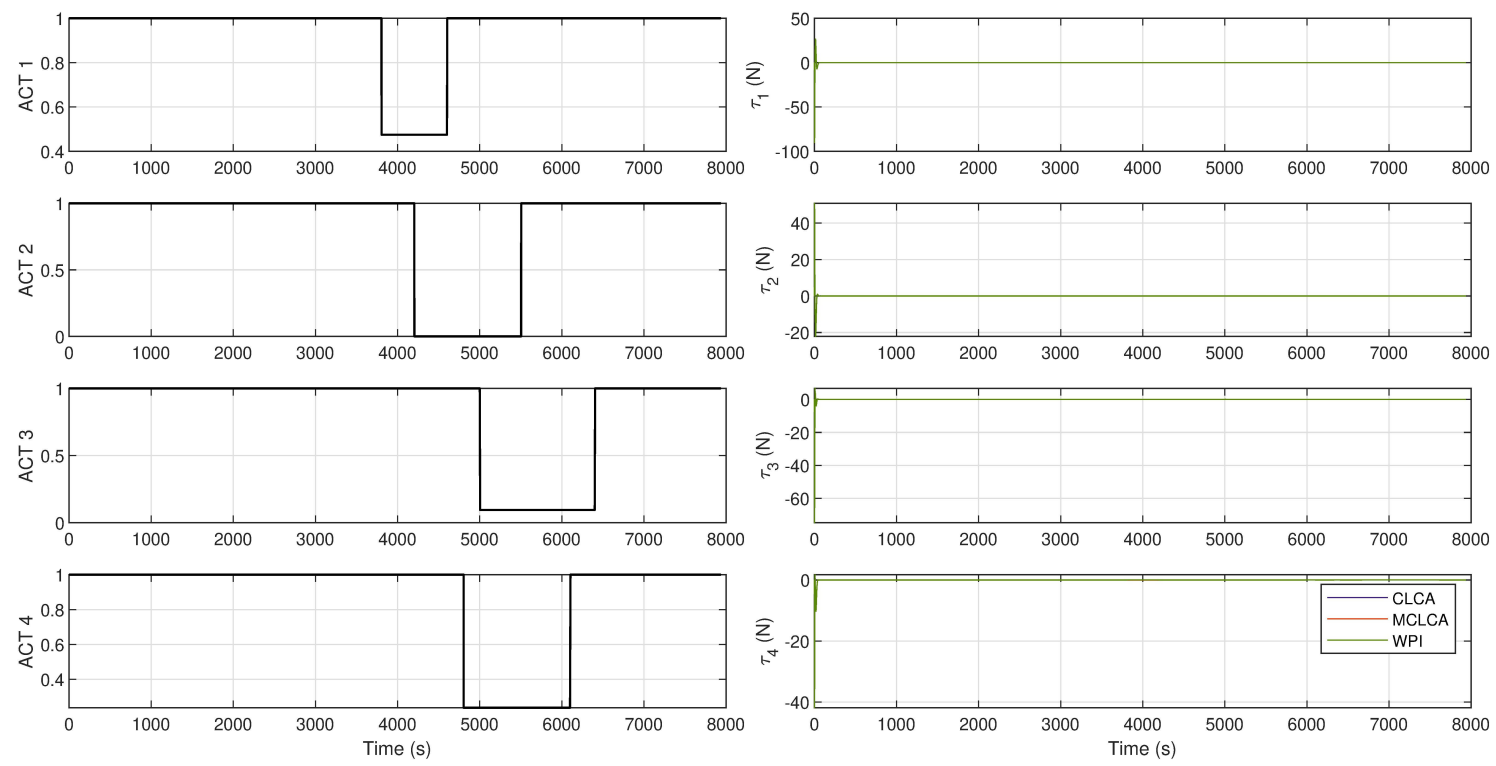

Figure 8.47: Fault Estimate

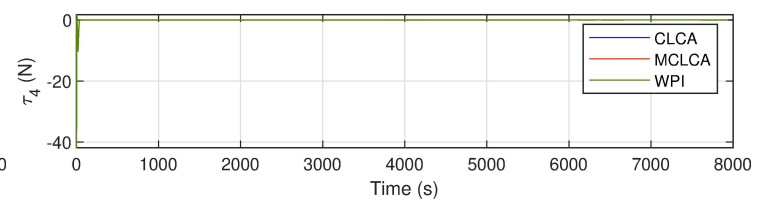

Figure 8.48: Actuator Forces 

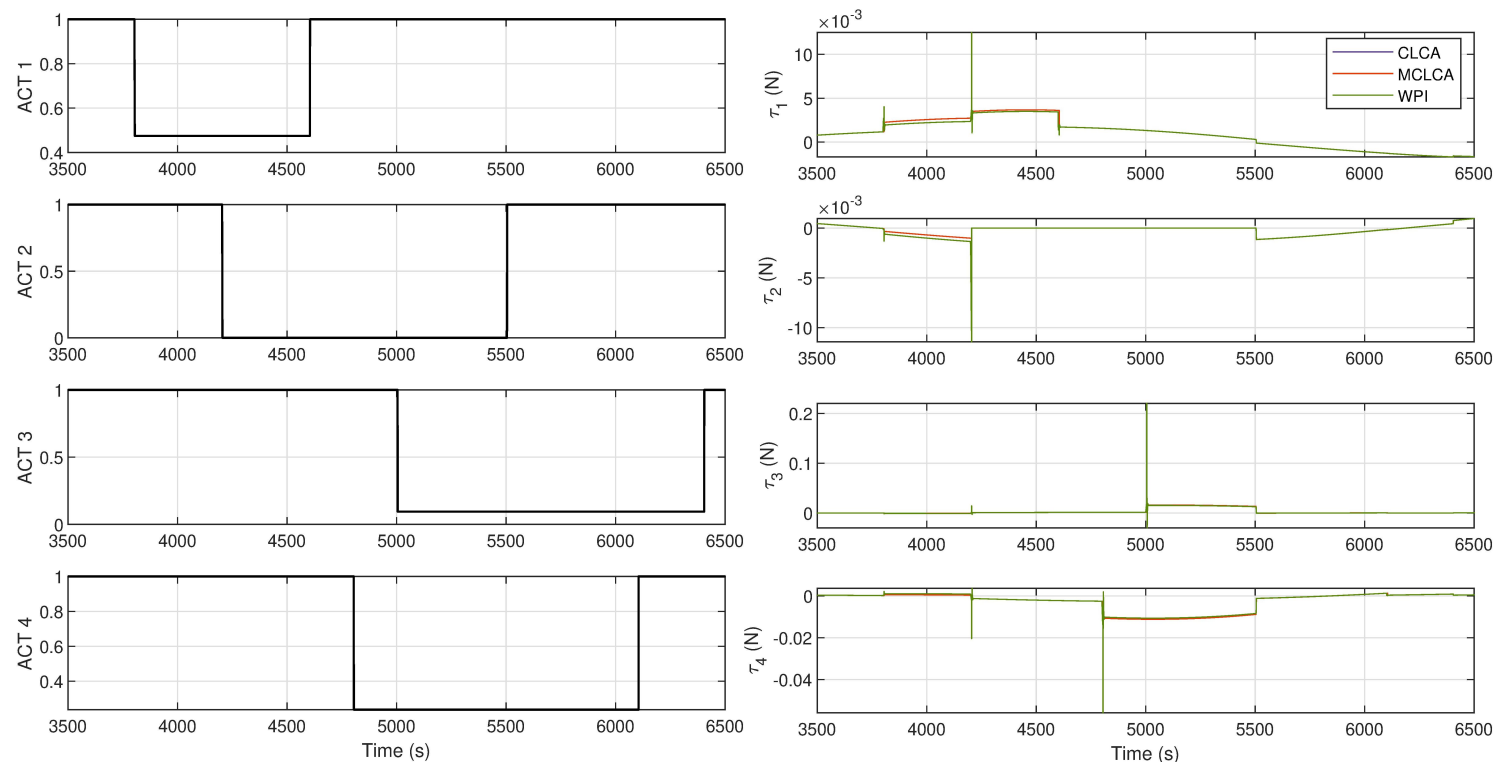

Figure 8.49: Fault Estimate

Figure 8.50: ACT Forces during Faults
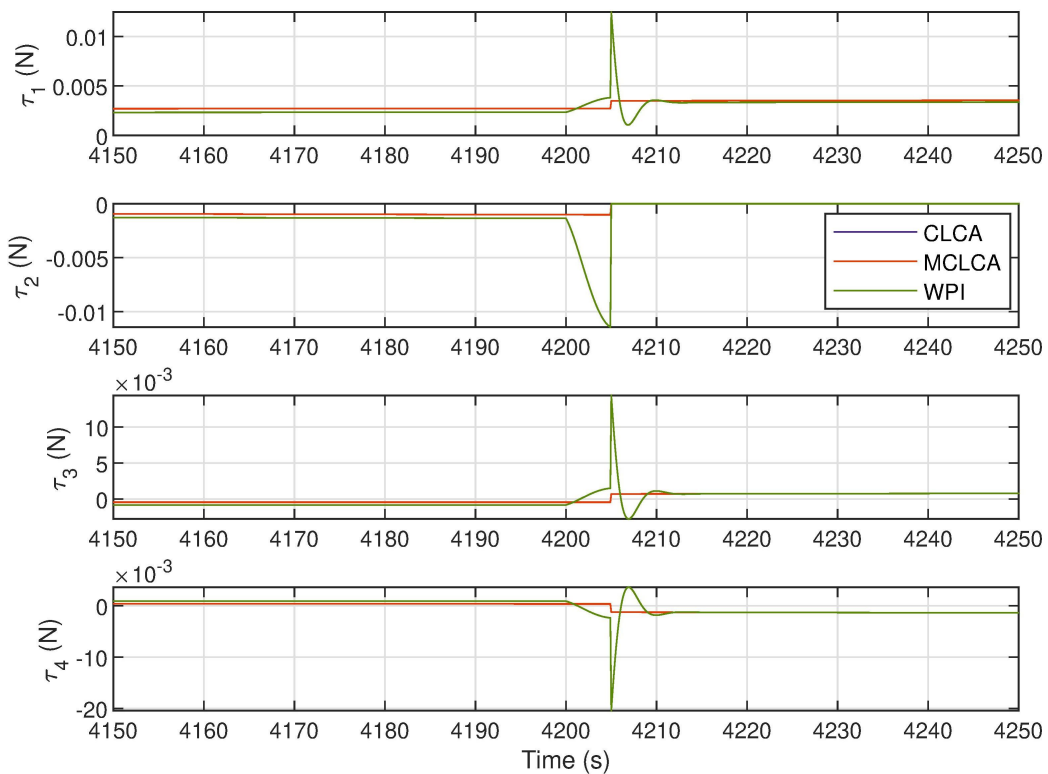

Figure 8.51: Actuator Forces at 4205 seconds 

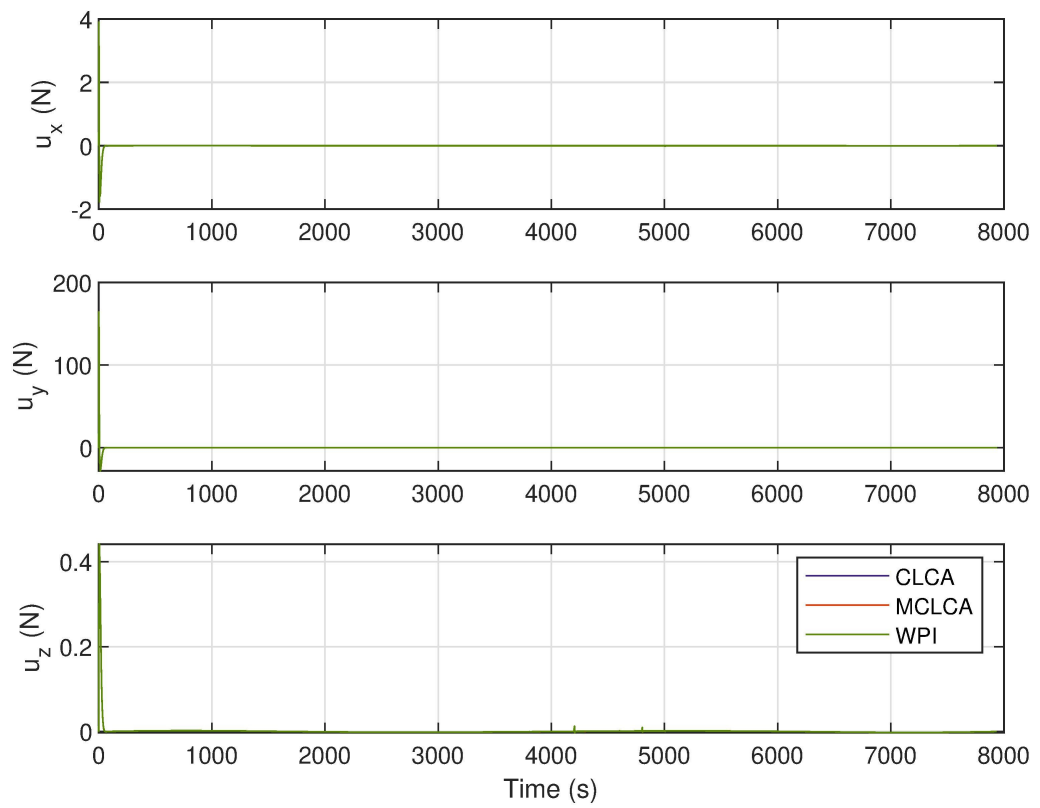

Figure 8.52: Control Force
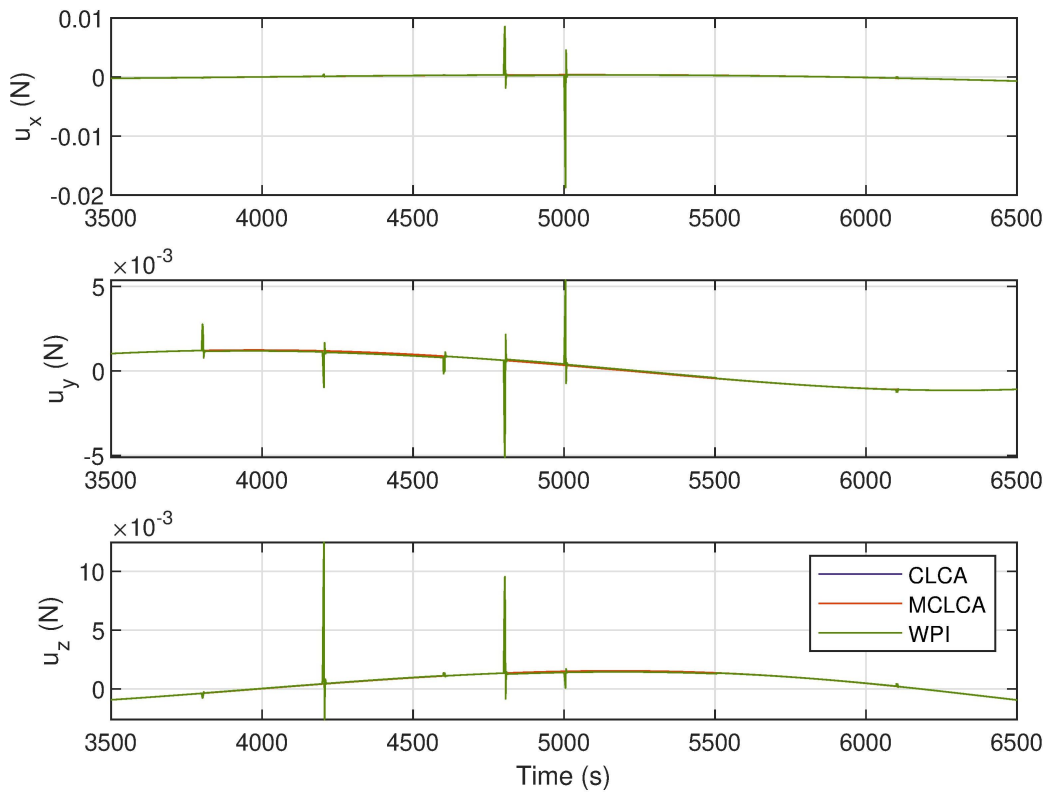

Figure 8.53: Control Force during Fault 

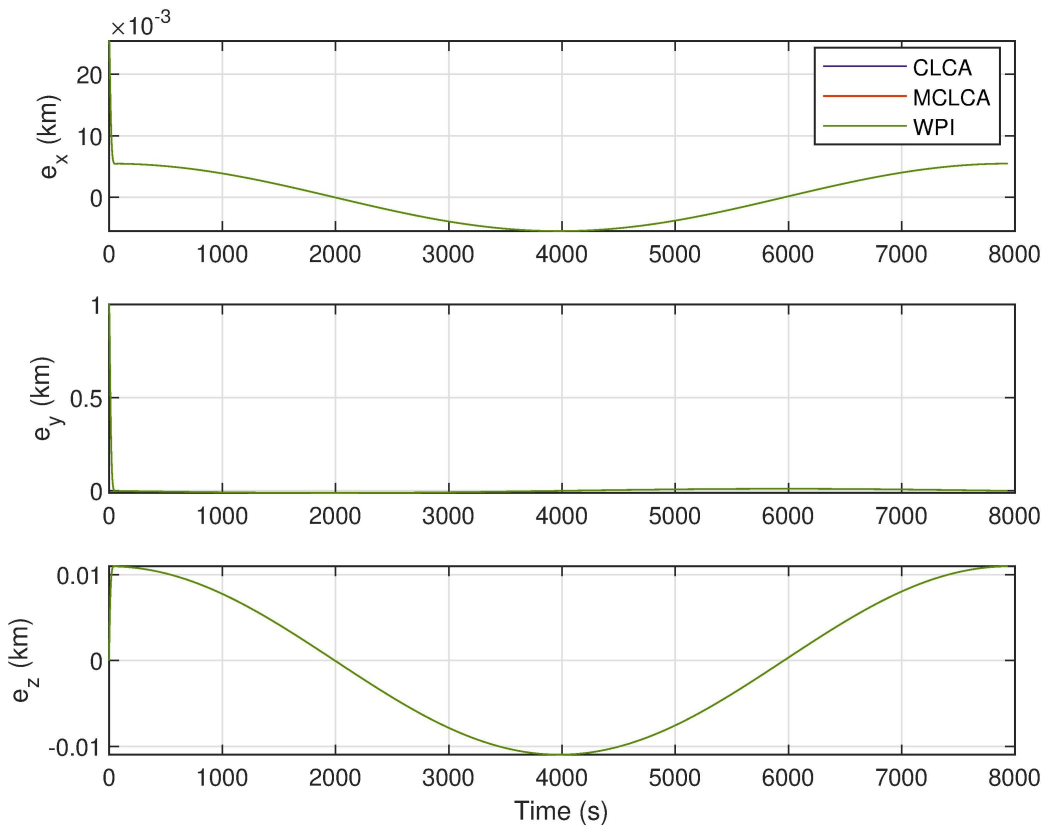

Figure 8.54: Position Error 
Figure 8.55 shows the actuator forces for only CLCA and MCLCA during the fault time frame. As seen here, the performance of the two algorithms are identical. From this graph, the conclusion can be made that even though [43] stated that MCLCA outperform CLCA for actuator uncertainties, the performance is the same under faults.
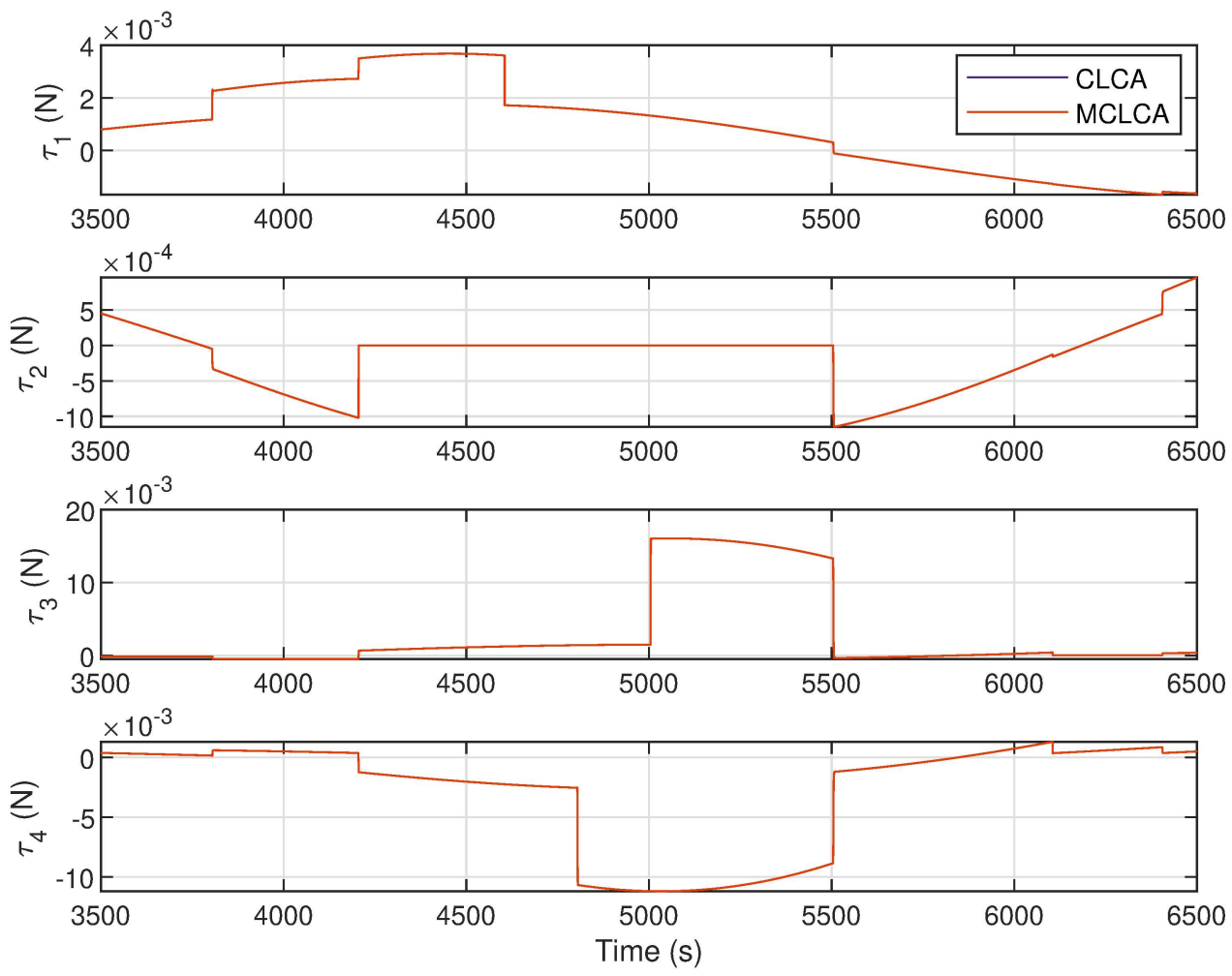

Figure 8.55: CLCA vs. MCLCA 


\section{Chapter 9}

\section{Conclusion}

This final chapter of the thesis summarizes the objectives and the results of the thesis, followed by a future work section.

\subsection{Thesis Summary}

Even thought spacecraft formation flying has be identified as a technology with plenty of potentials in the near future, a successful mission requires having a robust on-board GNC software. Given the highly nonlinear dynamical effects and the perturbations, the spacecraft could easily drift apart.

As mentioned in the earlier chapters, keeping a tight formation is critical for the majority if not all SFF missions so that the mission's objectives can be fulfilled. The goal of this research project was to develop an adaptive fault tolerant control law such that the formation is kept when the system experiences faults in a perturbed environment, so that the chaser spacecraft can accurately track a desired relative trajectory.

The performance of four control laws was studied. These four control laws were simple adaptive control (SAC), two types of nonsingular terminal sliding mode control, NTSMC and modified NTSMC (MNTSMC), and optimal sliding mode control (OSMC).

First the control laws were tested for a nominal scenario. In this scenario, it was observed that $\mathrm{SAC}$ has the fastest transient response and requires the least amount of control force. During steady state, it also had the smallest position error. Even though OSMC also had the same steady state error as SAC, SAC outperformed it during the transient phase.

A significant advantage of SAC compared to the three other control laws is that SAC is not a model based control law. That is, SAC does not require any knowledge of the plant, while the three sliding mode control laws do. In order to test the benefits of having a none model based control law, the chaser in the plant was 
subjected to extra perturbations with a very large magnitude. The results showed that SAC's performance in terms of position error was not affected by the extra perturbations. On the other hand, the position error of the three sliding mode control laws was significantly influenced by the perturbation. The conclusion is made that SAC, being a model-free control law, has a superior performance compared to model based control laws as an inaccuracy in the plant does not mitigate its performance.

In the last section of comparing the control laws, three different types of faults were implemented in the simulation. These were total loss of actuation, locked in place actuators, and partial loss of effectiveness. For the total loss of effectiveness, the three actuators failed for a duration of about 5500 seconds. While the actuators failed to produce any thrust, the two spacecraft drifted apart as expected. Once the actuators recovered from the faults, SAC had the fastest recovery rate at a cost of slightly higher control force compared to OSMC. OSMC had the second fastest recovery rate.

For the locked in place scenario, the three actuators got stuck at $[-2.2 \times$ $\left.10^{-4},-5.7 \times 10^{-4},-1.36 \times 10^{-3}\right]^{T} \mathrm{~N}$ for a duration of one orbit. Being stuck at these value caused an increase in the position error, with the largest in the y-axis. Once the actuators recovered, SAC again had the fastest recovery rate. However, this time it was at the cost of a much more required control force.

For the last scenario, the loss of effectiveness, the three actuators lost their effectiveness by $95 \% 30$ seconds into the mission. The performance of all control laws except for SAC degraded significantly; SAC's performance was not affected by the fault.

The second topic discussed in this thesis was control allocation (CA). CA is used to distribute the control effort to individual actuators in a specified manner. $\mathrm{CA}$ is often based on optimization techniques. In this thesis, three CA algorithms were implemented and compared with one another. These were weighted pseudo inverse and closed loop quadratic programming. Weighted pseudo inverse has been extensively used before for various systems, while closed loop quadratic programming is relatively new and has only been implemented for fault tolerant spacecraft attitude control. Two different fault scenarios were implemented in the numerical simulation so that a more concrete conclusion can be made about the performance of the different CA schemes. For both scenarios, the quadratic programming had a better performance. The CA was smoother, making quadratic programming a better choice. To add on, no differences were observed between the performance of CLCA and MCLCA. 


\section{$9.2 \quad$ Future Work}

Since simple adaptive control performed very well in the faulty scenarios, the performance of other adaptive control laws could also be tested for the three types of faults explained earlier. As example of this would be invariance and adaptive control (I\&I). To add on, other formation types and orbits such as highly elliptical orbit could also be tested for the three fault types.

For the CA section of this thesis, implementing an on-board fault detection and isolation would make the performance of the system more realistic and robust. In addition, a saturation limit could be implemented in the system to that the performance would become more realistic.

Lastly, testing both the fault and the CA algorithms at Carleton's Spacecraft Robotics and Control Laboratory GPS-Based Formation Flying Testbed would give a better understanding of the results of this thesis from an experimental perspective. 


\section{Bibliography}

[1] G. Bonin, N. Roth, S. Armitage, J. Newman, B. Risi, and R. E. Zee, "CanX-4 and CanX-5 Precision Formation Flight Mission Accomplished!" in 29th Annual AIAA/USU Conference on Small Satellites, 2015.

[2] D. Folta, J. Bristow, A. Hawkins, and G. Dell, "NASA's Autonomous Formation Flying Technology Demonstration, Earth Observing-1 (EO-1)," in International Symposium: Formation Flying Missions and Technology, 2002.

[3] D. Folta and D. Quinn, "Enhanced Formation Flying For The Earth Observing 1 (EO-1) New Millennium Mission," in Flight Mechanics Symposium, Greenbelt, MD, May 1997, pp. 405-406.

[4] G. D. Mauro, M. Lawn, and R. Bevilacqua, "Survey on Guidance Navigation and Control Requirements for Spacecraft Formation-Flying Missions," Journal of Guidance, Control, and Dynamics, vol. 41, no. 3, pp. 1-22, March 2018.

[5] D. Barret, T. Belloni, S. Bhattacharyya, M. Gilfanov, E. Gogus, J. Homan, M. Méndez, J. M. Miller, M. C. Miller, S. Mereghetti, S. Paltani, J. Poutanen, J. Wilms, and A. A. Zdziarski, "Science with the XEUS High Time Resolution Spectrometer," in Space Telescopes and Instrumentation 2008: Ultraviolet to Gamma Ray, vol. 7011, July 2008.

[6] J. Llorente, A. Agenjo, C. Carrascosa, C. de Negueruela, A. Mestreau-Garreau, A. Cropp, and A. Santovincenzo, "PROBA-3: Precise formation flying demonstration mission," Acta Astronautica, vol. 82, pp. 38-46, 2013.

[7] P. R. Lawson, "The Terrestrial Planet Finder," in 2001 IEEE Aerospace Conference Proceedings, vol. 4, Big Sky, MT, March 2001, pp. 4/2005-4/2011.

[8] M. Aung, A. Ahmed, M. Wette, J. Tien, D. Scharf, G. Purcell, B. Landin, and M. Regehr, "An Overview of Formation Flying Technology Development for the Terrestrial Planet Finder Mission," in 2004 IEEE Aerospace Conference Proceedings, vol. 4, Big Sky, MT, March 2004, pp. 2667-2679. 
[9] S. Persson, B. Jacobsson, and E. Gill, "PRISMA-Demonstration Mission for Advanced Rendezvous and Formation Flying Technologies and Sensors," in 56th International Astronautical Congress, Fukuoka, Japan, Oct. 2005.

[10] J. P. How, R. Twiggs, D. Weidow, K. Hartman, and F. Bauer, "Orion: A Lowcost Demonstration Of Formation Flying In Space Using GPS," in AIAA/AAS Astrodynamics Specialist Conference and Exhibit, 1998.

[11] R. Burns, C. A. McLaughlin, J. Leitner, and M. Martin, "TechSat 21: Formation Design, Control, and Simulation," in 2000 IEEE Aerospace Conference. Proceedings, vol. 7, Big Sky, MT, March 2000, pp. 19-25.

[12] C. Underwood, S. Pellegrino, V. J. Lappas, C. P. Bridges, and J. Baker, "Using CubeSat/micro-satellite technology to demonstrate the Autonomous Assembly of a Reconfigurable Space Telescope(AAReST)," Acta Astronautica, vol. 114, pp. 112-122, 2015.

[13] R. Kahle, B. Schlepp, S. Aida, M. Kirschner, and M. Wermuth, "Flight Dynamics Operations of the TanDEM-X Formation," in SpaceOps 2012, Stockholm, Schweden, June 2012.

[14] B. Robertson and E. Stoneking, "Satellite GNC Anomaly Trends," in 26th Annual AAS Guidance and Control Conference, Breckenridge, CO, Feb. 2003.

[15] National Aeronautics \& Space Administration, "Lewis Spins Out of Control," System Failure Case Studies, vol. 1, no. 8, pp. 1-4, Oct. 2007.

[16] T. A. Johansen and T. I. Fossen, "Control allocation-A survey," Automatica, vol. 49, pp. 1087-1103, 2013.

[17] H. Gui and A. H. J. de Ruiter, "Adaptive Fault-Tolerant Spacecraft Pose Tracking With Control Allocation," IEEE Transactions on Control Systems Technology, vol. 27, no. 2, pp. 479-494, March 2019.

[18] J. W. Kruk, B. F. Class, D. Rovner, J. Westphal, T. B. Ake, H. W. Moos, B. Roberts, and L. Fisher, "FUSE In-Orbit Attitude Control with Two Reaction Wheels and No Gyroscope," in Future EUV/UV and Visible Space Astrophysics Missions and Instrumentation, vol. 4854, Waikoloa, Hawai'i, Feb. 2003, pp. 274285.

[19] Godard and K. D. Kumar, "Fault Tolerant Reconfigurable Satellite Formations Using Adaptive Variable Structure Techniques," Journal of Guidance, Control, and Dynamics, vol. 33, no. 3, pp. 969-984, May-June 2010. 
[20] L. Cao, X. Chen, and A. K. Misra, "Minimum sliding mode error feedback control for fault tolerant reconfigurable satellite formations with J2 perturbations," Acta Astronautica, vol. 96, pp. 201-216, 2014.

[21] D. Lee, K. D. Kumar, and M. Sinha, "Fault detection and recovery of spacecraft formation flying using nonlinear observer and reconfigurable controller," Acta Astronautica, vol. 97, pp. 58-72, 2014.

[22] K. K. T. Thanapalan, S. M. Veres, E. Rogers, and S. B. Gabriel, "Fault Tolerant Controller Design to Ensure Operational Safety in Satellite Formation Flying," in Proceedings of the 45th IEEE Conference on Decision and Control, San Diego, CA, Dec. 2006, pp. 1562-1567.

[23] H. Dong, Q. Hu, and G. Ma, "Dual-quaternion based fault-tolerant control for spacecraft formation flying with finite-time convergence," ISA Transactions, vol. 61, pp. 87-94, 2016.

[24] Z. Zhu and Y. Guo, "Adaptive fault-tolerant attitude tracking control for spacecraft formation with unknown inertia," International Journal of Adaptive Control and Signal Processing, vol. 32, no. 1, pp. 13-26, Jan. 2018.

[25] D. Bustan, S. K. Hosseini Sani, and N. Pariz, "Adaptive Fault Tolerant Spacecraft Attitude Control Design with Transient Response Control," IEEE/ASME Transactions on Mechatronics, vol. 19, no. 4, pp. 1404-1411, Aug. 2014.

[26] B. Xiao, Q. Hu, Y. Zhang, and X. Huo, "Fault-Tolerant Tracking Control of Spacecraft with Attitude-Only Measurement Under Actuator Failures," Journal of Guidance, Control, and Dynamics, vol. 37, no. 3, pp. 838-849, May-June 2014 .

[27] H. Gui and G. Vukovich, "Adaptive fault-tolerant spacecraft attitude control using a novel integral terminal sliding mode," International Journal of Robust and Nonlinear Control, vol. 27, no. 16, pp. 3174-3196, Nov. 2017.

[28] Y. Omori, S. Suzuki, and K. Masui, "Flight Test of Fault-Tolerant Flight Control System using Simple Adaptive Control with PID Compensator," in Guidance, Navigation, and Control and Co-located Conferences, Boston, MA, Aug. 2013.

[29] R. Takase, J. O. Entzinger, and S. Suzuki, "Interaction between a Fault-Tolerant Flight Control System Using Simple Adaptive Control and Pilot's Pitch Control," in 2017 11th Asian Control Conference (ASCC), Broadbeach, Australia, Dec. 2017, pp. 507-512. 
[30] F. Chen, Q. Wu, B. Jiang, and G. Tao, "A Reconfiguration Scheme for Quadrotor Helicopter via Simple Adaptive Control and Quantum Logic," IEEE Transactions on Industrial Electronics, vol. 62, no. 7, pp. 4328-4335, July 2015.

[31] A. I. Belkharraz and K. Sobel, "Simple Adaptive Control for Aircraft Control Surface Failures," IEEE Transactions on Aerospace and Electronic Systems, vol. 43, no. 2, pp. 600-611, April 2007.

[32] A. Cano and K. Sobel, "Simple Adaptive Delta Operator Aircraft Flight Control for Accommodation of Loss of Control Effectiveness," City University of New York Academic Works, 2016.

[33] J. D. Bošković and R. K. Mehra, "Stable Multiple Model Adaptive Flight Control for Accommodation of a Large Class of Control Effector Failures," in Proceedings of the 1999 American Control Conference, vol. 3, San Diego, CA, June 1999, pp. 1920-1924.

[34] — - "Control Allocation in Overactuated Aircraft under Position and Rate Limiting," in Proceedings of the 2002 American Control Conference, vol. 1, Anchorage, AK, May 2002, pp. 791-796.

[35] M. Wang, J. Yang, G. Qin, and Y. Yan, "Adaptive Fault-tolerant Control with Control Allocation for Flight Systems with Severe Actuator Failures and Input Saturation," in 2013 American Control Conference, Washington, DC, June 2013, pp. 5134-5139.

[36] W. C. Arun Kishore, S. Sen, and G. Ray, "Disturbance Rejection and Control Allocation of Over-Actuated systems," in 2006 IEEE International Conference on Industrial Technology, Mumbai, India, Dec. 2006, pp. 1054-1059.

[37] M. Wang, J. Yang, and N. Li, "Adaptive Fault-Tolerant Control for Flight Systems with Input Saturation and Model Mismatch," Discrete Dynamics in Nature and Society, vol. 2013, 2013.

[38] H. Alwi and C. Edwards, "Fault tolerant control using sliding modes with on-line control allocation," Automatica, vol. 44, pp. 1859-1866, 2008.

[39] Q. Shen, D. Wang, S. Zhu, and E. K. Poh, "Inertia-free fault-tolerant spacecraft attitude tracking using control allocation," Automatica, vol. 62, pp. 114-121, 2015 .

[40] O. Härkegård, "Dynamic Control Allocation Using Constrained Quadratic Programming," Journal of Guidance, Control, and Dynamics, vol. 27, no. 6, pp. 1028-1034, Nov.-Dec. 2004. 
[41] Q. Hu, B. Li, and Y. Zhang, "Nonlinear Proportional-Derivative Control Incorporating Closed-Loop Control Allocation for Spacecraft," Journal of Guidance, Control, and Dynamics, vol. 37, no. 3, pp. 799-812, May-June 2014.

[42] B. Li, Q. Hu, G. Ma, and Y. Yang, "Fault-Tolerant Attitude Stabilization Incorporating Closed-Loop Control Allocation Under Actuator Failure," IEEE Transactions on Aerospace and Electronic Systems, vol. 55, no. 4, pp. 19892000, Aug. 2019.

[43] J. Qiao, Z. Liu, and W. Li, "Anti-disturbance attitude control of combined spacecraft with enhanced control allocation scheme," Chinese Journal of Aeronautics, vol. 31, no. 8, pp. 1741-1751, Aug. 2018.

[44] A. Imani and M. Bahrami, "Optimal sliding mode control for spacecraft formation flying in eccentric orbits," Proceedings of the Institution of Mechanical Engineers, Part I: Journal of Systems and Control Engineering, vol. 227, no. 5, pp. 474-481, May 2013.

[45] K. Alfriend, S. R. Vadali, P. Gurfil, J. P. How, and L. S. Breger, Spacecraft Formation Flying: Dynamics, control and navigation. Elsevier, 2010.

[46] A. H. J. de Ruiter, C. J. Damaren, and J. R. Forbes, Spacecraft Dynamics and Control: An Introduction. John Wiley \& Sons, 2013.

[47] W. Hu, Fundamental Spacecraft Dynamics and Control. John Wiley \& Sons, 2015.

[48] F. L. Markley and J. L. Crassidis, Fundamentals of Spacecraft Attitude Determination and Control. Springer, 2014.

[49] M. J. Sidi, Spacecraft Dynamics and Control: A Practical Engineering Approach. Cambridge University Press, 1997.

[50] C. A. Kluever, Space Flight Dynamics. John Wiley \& Sons, 2018.

[51] V. A. Chobotov, Orbital mechanics, 3rd ed. American Institute of Aeronautics and Astronautics, 2002.

[52] H. D. Curtis, Orbital Mechanics for Engineering Students. Elsevier Butterworth-Heinemann, 2005.

[53] W. M. Folkner, J. G. Williams, D. H. Boggs, R. S. Park, and P. Kuchynka, "The Planetary and Lunar Ephemerides DE430 and DE431," IPN Progress Report, Feb. 2014. 
[54] H. D. Curtis, Orbital Mechanics for Engineering Students, 3rd ed. Elsevier Butterworth-Heinemann, 2015, ch. 12.

[55] S. Ulrich, AERO 4540: Spacecraft Attitude Dynamics and Control, Carleton University, Ottawa, ON, Fall 2018.

[56] O. Montenbruck and E. Gill, Satellite Orbits: Models, Methods and Applications, 1st ed. Springer, 2000.

[57] D. A. Vallado, Fundamentals of Astrodynamics and Applications, 2nd ed. Space Technology Library, 2001, ch. 8.

[58] H. K. Khalil, Nonlinear Control. Pearson Education Limited, 2015.

[59] Y. Feng, X. Yu, and Z. Man, "Non-singular terminal sliding mode control of rigid manipulators," Automatica, vol. 38, pp. 2159-2167, 2002.

[60] J. Liu and X. Wang, Advanced Sliding Mode Control for Mechanical Systems: Design, Analysis and MATLAB Simulation. Springer, 2011.

[61] K. Hassani and W.-S. Lee, "Optimal Tuning of Linear Quadratic Regulators Using Quantum Particle Swarm Optimization," in Proceedings of the International Conference of Control, Dynamic Systems, and Robotics, no. 19, Ottawa, Canada, May 2014.

[62] M. A. Johnson and M. J. Grimble, "Recent trends in linear optimal quadratic multivariable control system design," in IEE Proceedings D (Control Theory and Applications), vol. 134, no. 1, Jan. 1987, pp. 53-71.

[63] W.-J. Cao and J.-X. Xu, "Nonlinear Integral-Type Sliding Surface for Both Matched and Unmatched Uncertain Systems," IEEE Transactions on Automatic Control, vol. 49, no. 8, pp. 1355-1360, Aug. 2004.

[64] K. Sobel, H. Kaufman, and L. Mabius, "Implicit Adaptive Control for a Class of MIMO Systems," IEEE Transactions on Aerospace and Electronic Systems, vol. 18 , no. 5, pp. 576-590, Sept. 1982.

[65] I. Bar-Kana and H. Kaufman, "Direct Adaptive Control with Bounded Tracking Errors," in The 22nd IEEE Conference on Decision and Control, San Antonio, TX, Dec. 1983, pp. 221-222.

[66] I. Bar-Kana, "Positive Realness in Discrete-Time Adaptive Control Systems," in 1986 American Control Conference, Seattle, WA, June 1986, pp. 1440-1443. 
[67] L. Hsu and R. R. Costa, "MIMO direct adaptive control with reduced prior knowledge of the high frequency gain," in Proceedings of the 38th IEEE Conference on Decision and Control, vol. 4, Phoenix, AZ, Dec. 1999, pp. 3303-3308.

[68] I. Barkana, "Output feedback stabilizability and passivity in nonstationary and nonlinear systems," International Journal of Adaptive Control and Signal Processing, vol. 24, no. 7, p. 568-591, July 2010.

[69] S. Ulrich, "Nonlinear Passivity-Based Adaptive Control of Spacecraft Formation Flying," in 2016 American Control Conference (ACC), Boston, MA, July 2016, pp. $7432-7437$.

[70] I. Barkana, "On Robustness and Perfect Tracking with Simple Adaptive Control in Nonlinear Systems," International Federation of Automatic ControlPapersOnLine, vol. 50, no. 1, pp. 4258-4263, July 2017.

[71] — - "Classical and Simple Adaptive Control for Nonminimum Phase Autopilot Design," Journal of Guidance, Control, and Dynamics, vol. 28, no. 4, pp. 631638, July-Aug. 2005.

[72] — - "Simple Adaptive Control: The Optimal Model Reference - Short tutorial," in 11th IFAC International Workshop on Adaptation and Learning in Control and Signal Processing, Caen, France, July 2013, pp. 396-407.

[73] D. Huston, B. Esser, G. Spencer, D. Burns, and E. Kahn, "Hierarchical Actuator Systems," in Smart Structures and Materials 2005: Industrial and Commercial Applications of Smart Structures Technologies, vol. 5762. International Society for Optics and Photonics, May 2005, pp. 311-319.

[74] M. T. Hamayun, C. Edwards, and H. Alwi, "Design and Analysis of an Integral Sliding Mode Fault-Tolerant Control Scheme," IEEE Transactions on Automatic Control, vol. 57, no. 7, pp. 1783-1789, July 2012.

[75] J. Wang and R. G. Longoria, "Coordinated Vehicle Dynamics Control with Control Distribution," in Proceedings of the 2006 American Control Conference, Minneapolis, MN, June 2006, pp. 5348-5353.

[76] A. B. Page and M. L. Steinberg, "A Closed-loop Comparison of Control Allocation Methods," in AIAA Guidance, Navigation, and Control Conference and Exhibit, Denver, CO, Aug. 2000.

[77] Y. Zhang and Z. Chen, "A Closed-loop Control Allocation Method for Satellite Precision Pointing," in IEEE 10th International Conference on Industrial Informatics, Beijing, China, July 2012, pp. 1108-1112. 
[78] O. Härkegård, "Backstepping and Control Allocation with Applications to Flight Control," Ph.D. dissertation, Department of Electrical Engineering, Linköping University, Linköping, Sweden, 2003. 